\title{
Vital imaging of large arteries using two-photon laser scanning microscopy : focus on the arterial wall
}

Citation for published version (APA):

Megens, R. T. A. (2008). Vital imaging of large arteries using two-photon laser scanning microscopy : focus on the arterial wall. [Doctoral Thesis, Maastricht University]. Universiteit Maastricht. https://doi.org/10.26481/dis.20080314rm

Document status and date:

Published: 01/01/2008

DOI:

10.26481/dis.20080314rm

Document Version:

Publisher's PDF, also known as Version of record

\section{Please check the document version of this publication:}

- A submitted manuscript is the version of the article upon submission and before peer-review. There can be important differences between the submitted version and the official published version of record.

People interested in the research are advised to contact the author for the final version of the publication, or visit the DOI to the publisher's website.

- The final author version and the galley proof are versions of the publication after peer review.

- The final published version features the final layout of the paper including the volume, issue and page numbers.

Link to publication

\footnotetext{
General rights rights.

- You may freely distribute the URL identifying the publication in the public portal. please follow below link for the End User Agreement:

www.umlib.nl/taverne-license

Take down policy

If you believe that this document breaches copyright please contact us at:

repository@maastrichtuniversity.nl

providing details and we will investigate your claim.
}

Copyright and moral rights for the publications made accessible in the public portal are retained by the authors and/or other copyright owners and it is a condition of accessing publications that users recognise and abide by the legal requirements associated with these

- Users may download and print one copy of any publication from the public portal for the purpose of private study or research.

- You may not further distribute the material or use it for any profit-making activity or commercial gain

If the publication is distributed under the terms of Article $25 \mathrm{fa}$ of the Dutch Copyright Act, indicated by the "Taverne" license above, 


\section{Vital Imaging of Large Arteries using Two-Photon Laser Scanning Microscopy: Focus on the Arterial Wall}


ISBN 978-90-9022535-7

(C) Copyright R.T.A. Megens, Maastricht 2008

Cover image: Collagen in the tunica adventitia of a mouse carotid artery visualized with two-photon laser scanning microscopy, second harmonic generation of collagen, and CNA35/OG488.

Lay-out and cover design by Hans en Inge Duimel Printed by Drukkerii Duplica

Financial support for printing of this thesis was kindly provided by AstraZeneca R\&D, Department of Bioscience, Mölndal (Sweden).

\section{AstraZeneca}




\title{
Vital Imaging of Large Arteries using Two-Photon Laser Scanning Microscopy: Focus on the Arterial Wall
}

\author{
PROEFSCHRIFT
}

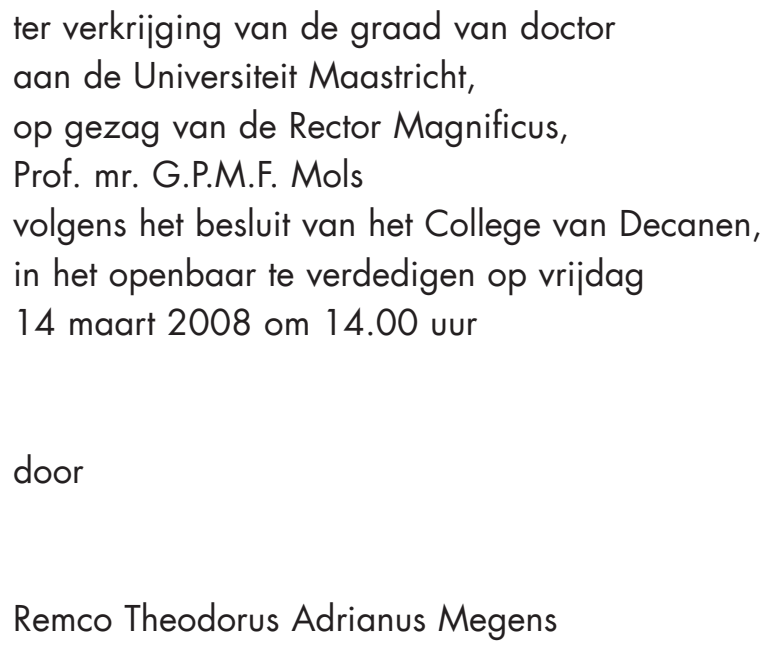




\section{Promotor}

Prof. dr. D.W. Slaaf

\section{Copromotores}

dr. M.A.M.J. van Zandvoort

dr. M.G.A. oude Egbrink

Beoordelingscommissie

Prof. dr. H.A.J. Struijker Boudier (voorzitter)

Prof. dr. M.J.A.P. Daemen

Prof. dr. H.C. Gerritsen (Universiteit Utrecht)

Prof. dr. P.J.E.H.M. Kitslaar

Prof. dr. C. Weber (Universitätsklinikum Aachen) 
Aftrap

Als de trap de hoogste trede heeft gehaald, blijkt de behoefte om te stijgen al gedaald.

Albert Megens

(uit: 'Tussen aftrap en opstap', 1999) 



\section{Contents}

Chapter 1 General Introduction

Chapter 2 Large Arteries, Atherosclerosis, and Two-Photon Laser Scanning Microscopy

Chapter 3 Two-Photon Microscopy of Vital Murine Elastic and Muscular Arteries: Combined Structural and Functional Imaging with Subcellular Resolution.

Chapter 4 Imaging Collagen in Intact Viable Healthy and CNA35 and Two-Photon Laser Scanning Microscopy

Chapter 5 Imaging Collagen and Inflammatory Cells in Atherosclerotic Plaques in Vital Carotid Arteries using Two-Photon Microscopy

Chapter 6 Imaging Enhanced NO levels in Stimulated Sprouting Cultured Endothelial Cells

Chapter 7 In Vivo Imaging of Murine Large Arteries using Two-Photon Laser Scanning Microscopy:

a Feasibility Study

Chapter 8 General Discussion

Chapter 9 Summary

Samenvatting

Dankwoord

Curriculum Vitae

List of Publications 



\section{Chapter 1}

General Introduction 
Chapter 1 
The circulation is a complex system consisting of different types of blood vessels. It is now recognized that these vessels are part of a subtle regulatory system with differential properties along the vascular tree. Alterations in the vessel wall or malfunctioning of blood vessels may lead to various diseases such as atherosclerosis or hypertension.

This thesis focuses on the vessel wall of large murine arteries and cultured cell systems to evaluate the applicability of two-photon laser scanning microscopy as an imaging tool for studying alterations in vessel wall properties and their functional consequences.

The wall of large arteries consists of three layers that are separated by elastic bands: the inner layer or tunica intima, the medial layer or tunica media, and the outer layer or tunica adventitia. Each layer has its specific morphology and function. The tunica intima consists of one layer of endothelial cells that is supported by a thin basement membrane. Although only one layer thick, the endothelial cells are functionally important. They are at the interface between the vascular wall and the circulating blood, where they play a key role in control of blood cell-vessel wall interactions [1-3], vascular tone, and vascular permeability [4].The tunica media consists of several layers of vascular smooth muscle cells (separated by elastin bands) which regulate vascular tone. The tunica adventitia contains large amounts of connective tissue which provide the artery with tensile strength. Furthermore, nerve fibers are present for innervation of the vascular smooth muscle cells. Especially in thick or diseased arteries, a vasa vasorum (a network of small capillaries, arterioles, and venules) is present to provide nutrients and oxygen to the tunica adventitia and media and to remove metabolites and waste products.

Alterations of structural and functional properties of the arterial wall may lead to vascular diseases, such as atherosclerosis.

In atherosclerosis, lesions develop at lesion-prone sites, such as bifurcations. At these sites, lipids and inflammatory cells from the bloodstream penetrate through the endothelial cell layer and accumulate in the subendothelial space in the tunica intima. In time, these initial fatty streaks progress towards more advanced stages of atherosclerosis. Rupture of such atherosclerotic plaques may eventually lead to thromboembolic complications [3, 5-7]. Thrombi may occlude the lumen, and emboli may cause downstream ischemia. For this reason, atherosclerosis is one of the major causes for heart failure or stroke in the western world [6].

During the last few decades, histology has provided detailed insight in various 
aspects of blood vessel morphology and atherosclerosis in human and murine samples. However, preparation of histological sections requires extensive chemical treatment and slicing of the tissue, which results in loss of viability and functionality and may result in structural alterations [8]. This precludes insight in the structure of plaque components under physiological circumstances and in functional properties of such structures in (diseased) arteries. Further understanding of the (diseased) vasculature requires such better insight in structure and function of the vascular wall at subcellular level. An imaging technique which enables simultaneous visualization of both structure and function of the intact and viable diseased vascular wall of large arteries is therefore essential. To achieve the required contrast and signal necessary for imaging, fluorescent markers are required that are specific for certain structures. These markers must be applicable in viable samples, which are less permeable and therefore limit penetration of these markers into the tissue (unlike histology where fixatives make the samples more permeable). In addition, viability of the sample should not be affected by the markers.

In 1990, two-photon laser-scanning microscopy (TPLSM) was developed by Webb and colleagues [9, 10]. TPLSM is based on the principle of two-photon excitation, a process which already was described in 1931 by Maria GöppertMayer [10]. Simultaneous absorption of two near-infrared photons (total energy is equivalent to that of a single photon at half the wavelength) leads to the excited state of fluorescent molecules in the sample. Since the probability of two-photon absorption depends on the square of the intensity of the excitation light, excitation only occurs in a very small volume at focal position of the microscope. Therefore, out of focus absorption and excitation, as in normal or confocal fluorescence microscopy, are absent and as a result, emitted photons always originate from the focal position and thus are independent of scattering. Due to this excitation process, TPLSM possesses some interesting features; enhanced depth penetration, good optical sectioning, good resolution in three dimensions, and strong reduction of photobleaching, photo-damage, and photo-toxicity [11]. This combination of features makes TPLSM better than other microscopic techniques for imaging of structures which are located deep in (scattering) tissues such as brain [12], kidney [13, 14], microvasculature [15], and lymphatics [16].

Recently, TPLSM was established as a valuable tool for imaging of blood vessels. However, the blood vessels studied were very small [17], still treated with a fixative [18], sliced [19, 20], casted in hot (>40 $\left.{ }^{\circ} \mathrm{C}\right)$ agarose gel [21], or kept under non-physiological pressures [22], resulting in structural alterations and loss of functionality [8]. 
The studies described in the present thesis were performed in order to visualize a variety of morphological and functional properties of healthy and atherosclerosis-prone large arteries of mice using TPLSM. Furthermore, various fluorescent markers were evaluated on their specificity, viability, and penetration into the arterial wall.

As an introduction to the experimental chapters, chapter 2 gives a brief survey on the present and most relevant knowledge on imaging of blood vessels, vessel wall, and TPLSM imaging, which are all of interest for this thesis.

Chapter 3 of this thesis describes and evaluates a method that was developed to enable structural and functional imaging of intact and viable arteries. Dissected carotid and mesenteric arteries were mounted in a home-built perfusion chamber and pressurized up to physiological pressure. Characteristics of TPLSM imaging of mounted large arteries and viability of such arteries were evaluated. The structure of elastic and muscular arteries was compared, the very delicate endothelial glycocalyx was visualized, and functional properties of muscular arteries were assessed.

In Chapter 4, the method was adapted to visualize atherosclerotic lesions in the carotid artery bifurcation. The distribution of collagen was assessed in mounted viable large arteries of control (C57BL6/J) and atherosclerotic ( $\mathrm{ApoE}-1)$ mice using the novel fluorescent collagen marker CNA35/OG488. The uptake of CNA35/OG488 in mounted healthy large arteries appeared to be limited by an intact endothelial layer and elastic laminae. However, in mounted atherosclerotic arteries, CNA35/OG488 did penetrate into the vessel wall and labeled the collagen structure in atherosclerotic lesions. Intravenous administration of CNA35/OG488 in living mice further revealed that CNA35/OG488 might have potential as a molecular imaging agent.

Chapter 5 describes the visualization of atherosclerotic lesions in the carotid artery bifurcation and the direct relationship between collagen and inflammatory cells in intact and viable mounted carotid arteries of control and atherosclerotic mice, at a subcellular level. Development of atherosclerotic plaques was visualized from the initial phase towards more advanced lesions in the carotid bifurcation. Special attention was paid to the fibrous cap regions and the various vessel wall layers.

To illustrate the applicability of our technique to functional studies on blood vessels, we studied nitric oxide (NO) production. Up till now most knowledge concerning the role of $\mathrm{NO}$ in arteries is based on indirect measurements. The importance of $\mathrm{NO}$ was never directly established in viable arteries by visualizing NO production in the vessel wall. To this end, a fluorescent probe is needed which relates changes in the presence of NO with changes in fluorescent signal. Furthermore, since NO exist for only very short periods of 
time, such probe must be highly sensitive. Chapter 6 describes the first results of a novel NO-marker FNOCT in endothelial cells in vitro, which in potential can also be applied in large arteries.

In chapter 7, the potential of TPLSM for imaging of large arteries in vivo is studied. Good optical sectioning, as in TPLSM, is very sensitive to motional disturbances which occur as a consequence of heartbeat and respiration. To minimize the effects of these motional disturbances, we have reduced the exposure time of single optical sections. Furthermore, image acquisition was performed during a fixed period of the cardiac cycle by triggering the start of image acquisition. These trigger pulses were derived from the respiration- and blood pressure signal.

Chapter 8 is a general discussion in which the findings of the various chapters are discussed.

\section{References}

1. Muller, W.A., Leukocyte-endothelial cell interactions in the inflammatory response. Lab Invest, 2002;82(5):521-33.

2. Ebnet, K., E.P. Kaldjian, A.O. Anderson, et al., Orchestrated information transfer underlying leukocyte endothelial interactions. Annu Rev Immunol, 1996; 14:155-77.

3. oude Egbrink, M.G., M.A. Van Gestel, M.A. Broeders, et al., Regulation of microvascular thromboembolism in vivo. Microcirculation, 2005;12(3):287-300.

4. Cines, D.B., E.S. Pollak, C.A. Buck, et al., Endothelial cells in physiology and in the pathophysiology of vascular disorders. Blood, 1998;91(10):3527-61.

5. Stary, H.C., A.B. Chandler, R.E. Dinsmore, et al., A definition of advanced types of atherosclerotic lesions and a histological classification of atherosclerosis. A report from the Committee on Vascular Lesions of the Council on Arteriosclerosis, American Heart Association. Circulation, 1995;92(5):1355-74.

6. Lusis, A.J., Atherosclerosis. Nature, 2000;407(6801):233-41.

7. Libby, P., Changing concepts of atherogenesis. J Intern Med, 2000;247(3):349-58.

8. Dorph-Petersen, K.A., J.R. Nyengaard, and H.J. Gundersen, Tissue shrinkage and unbiased stereological estimation of particle number and size. J Microsc, $2001 ; 204(\mathrm{Pt} 3): 232-46$.

9. Denk, W., J.H. Strickler, and W.W. Webb, Two-photon laser scanning fluorescence microscopy. Science, 1990;248(4951):73-6.

10. Masters, B.R. and P.T. So, Antecedents of two-photon excitation laser scanning microscopy. Microsc Res Tech, 2004;63(1):3-11.

11. Konig, K., Multiphoton microscopy in life sciences. J Microsc, 2000;200 ( Pt 2):83-104.

12. Svoboda, K., W. Denk, D. Kleinfeld, et al., In vivo dendritic calcium dynamics in neocortical pyramidal neurons. Nature, 1997;385(6612):161-5.

13. Ashworth, S.L. and G.A. Tanner, Fluorescent labeling of renal cells in vivo. Nephron Physiol, 2006;103(2):p91-6. 
14. Dunn, K.W. and P.A. Young, Principles of multiphoton microscopy. Nephron Exp Nephrol, 2006; 103(2):e33-40.

15. Padera, T.P., B.R. Stoll, P.T. So, et al., Conventional and high-speed intravital multiphoton laser scanning microscopy of microvasculature, lymphatics, and leukocyte-endothelial interactions. Mol Imaging, 2002;1(1):9-15.

16. Worbs, T., T.R. Mempel, J. Bolter, et al., CCR7 ligands stimulate the intranodal motility of $T$ lymphocytes in vivo. J Exp Med, 2007;204(3):489-95.

17. Martinez-Lemus, L.A., M.A. Hill, S.S. Bolz, et al., Acute mechanoadaptation of vascular smooth muscle cells in response to continuous arteriolar vasoconstriction: implications for functional remodeling. Faseb J, 2004;18(6):708-10.

18. Ferrara, D.E., D. Weiss, P.H. Carnell, et al., Quantitative 3D fluorescence technique for the analysis of en face preparations of arterial walls using quantum dot nanocrystals and twophoton excitation laser scanning microscopy. Am J Physiol Regul Integr Comp Physiol, 2006;290(1):R1 14-23.

19. Boulesteix, T., A.M. Pena, N. Pages, et al., Micrometer scale Ex Vivo multiphoton imaging of unstained arterial wall structure. Cytometry A, 2006;69(1):20-6.

20. Parasassi, T., W. Yu, D. Durbin, et al., Two-photon microscopy of aorta fibers shows proteolysis induced by LDL hydroperoxides. Free Radic Biol Med, 2000;28(1 1):1589-97.

21. van Zandvoort, M., W. Engels, K. Douma, et al., Two-photon microscopy for imaging of the (atherosclerotic) vascular wall: a proof of concept study. J Vasc Res, 2004;41(1):54-63.

22. Maffia, P., B.H. Zinselmeyer, A. lalenti, et al., Images in cardiovascular medicine. Multiphoton microscopy for 3-dimensional imaging of lymphocyte recruitment into apolipoprotein-Edeficient mouse carotid artery. Circulation, 2007;115(11):e326-8. 
Chapter 1 


\title{
Chapter 2
}

\author{
Large Arteries, Atherosclerosis, and \\ Two-Photon Laser Scanning Microscopy
}


Chapter 2 
Atherosclerosis is a systemic disease of large arteries that slowly progresses with age and, affects virtually all persons to some extent. The development of atherosclerotic plaques, also called atherogenesis, already starts in early childhood [1, 2]. However, at such young ages, no clinically relevant symptoms are present. At the fifth or sixth decade of life the atherosclerotic process becomes clinically relevant (or earlier, dependent on several risk factors such as smoking, diabetes, hypertension, and high-cholesterol blood levels) [1-3]. A broad range of (severe) clinical complications may occur dependent on which arterial beds are affected. Atherosclerosis is the primary cause of cardiovascular events and stroke, and the underlying cause of approximately $50 \%$ of all deaths in western society $[4,5]$.

The last few decades, extensive research in the field of atherosclerosis resulted in better insight in the initiation and progression of atherogenesis. However, many questions remain to be answered. The acquired knowledge is mostly based on histological studies of isolated samples that have lost viability. For that reason, the functional properties of various compounds in the atherosclerotic vessel wall are still largely unknown. Better understanding of the functionality of the atherosclerotic arterial wall, and, thus, increased insight in (development of) atherosclerotic lesions requires studying of these properties in vivo or in still viable arteries ex vivo. This may lead to better understanding of the process of atherogenesis and the resulting complications and, in the end, enable better treatment and reduce the incidence and mortality.

In this chapter, a brief survey is given of the biology of large arteries and the process of atherosclerosis; in addition, animal models are described that can be used to study atherosclerosis. Then, the basic principles of conventional (single-photon) and two-photon fluorescence microscopy are discussed and compared. In addition, a short overview is dedicated to the fluorescent labeling of vascular structures. Finally, the current status of the applications of twophoton laser scanning microscopy for visualization of various aspects of larger arteries is discussed.

\section{Vessel wall structure of healthy large arteries}

The arterial wall is composed of three layers or tunicae (fig. 1, see also chapter 3) [6].

The tunica intima or inner layer is relatively thin and mainly consists of a single layer of endothelial cells that lines the vessel lumen. It is now recognized that the endothelial cells have an important regulatory function for several biological processes [7], rather than being a passive barrier between blood 
and the tissue. The function of endothelial cells differs considerably throughout the vasculature, not only between different organs but also between large and small blood vessels [8] and between vessels at the arterial and venous side of the circulation [9]. At its luminal side, the endothelium is covered by a functionally important thin network of membrane-bound macromolecules called the endothelial glycocalyx [10]. The glycocalyx is involved in many processes such as vascular permeability and mechanotransduction of forces exerted by blood flow. It controls interactions with a large number of molecules [10], contributing to the vasculoprotective properties of the vessel wall [11, 12]. At the abluminal side of the endothelial cells, a thin layer of connective tissue forms the basal membrane that supports the endothelial cells. The most abluminal part of the tunica intima is the internal elastic lamina (IEL) which separates it from the tunica media. The IEL is composed of a fenestrated layer of elastin. The number of fenestrae differs between arteries. These fenestrae contribute to cell-cell contact (via gap junctions [13-15] between endothelial cells and vascular smooth muscle cells (vSMCs) and may also allow diffusion of substances [6].

The tunica media or medial layer primarily consists of several concentric layers of helically arranged vascular smooth muscle cells. The number of layers depends on size and type of the artery. The vascular smooth muscle cells are separated by fibers of extracellular matrix (collagen, elastin). Interposed between these layers of vascular smooth muscle cells are intermediate elastic laminae (the number of intermediate laminae again depends on the size and type of the artery). The main function of the tunica media is control of vascular tone. Moreover, the vascular smooth muscle cells are the cellular source of the extracellular matrix $[6,16]$. The external elastic lamina (EEL) is the most outer elastic laminae of the tunica media and separates it from the tunica adventitia; it is composed of many elastin fibers that form a finely woven structure.

The tunica adventitia is the outer most layer of the arterial vessel wall and mainly consists of collagen and elastin fibers [6]. In between these fibers, fibroblast-like cells and vasomotor nerves are present. In the tunica adventitia of large arteries with a vessel wall that is too thick to be solely nourished by diffusion, a network of branched small blood vessels or vasa vasorum is present that provides metabolites and oxygen to the tunica adventitia and abuminal parts of the tunica media $[6,17,18]$.

The vessel walls of various large arteries have a number of structural features in common although structural variations between comparable arteries can be considerable. Note that thickness, detailed structure, and function of these three tunicae strongly depend on total diameter, type of blood vessel, and species. 
Based on their diameter and location, large arteries may be classified as elastic arteries (largest arteries) or muscular arteries (medium sized arteries). Elastic arteries are located directly downstream of the heart where blood is transported under high (pulsating) pressure. The vessel wall of elastic arteries is thick and elastic, and has a buffering function reducing the pulsations of blood flow. Muscular arteries are located more closely to organs and tissues and mainly have a conductive function and control the affluence of blood to the various organs [6]. The vessel wall of muscular arteries is thinner and their diameter (and, hence, resistance) is controlled by contracting or relaxing the vascular smooth muscle cells in the tunica media [6].

\section{Atherosclerosis}

\section{Atherogenesis}

Atherosclerosis leads to alterations of structural and functional properties of the vascular wall and was initially considered to be a lipid storage disease [19]. It is now recognized that the development and progression of atherosclerotic lesions is an inflammatory disease $[5,20,21]$.

Atherosclerotic lesions are asymmetric focal thickenings of the tunica intima [22]. Atherosclerotic lesion development (fig. 2) preferentially occurs in branch points or curves in the large arteries where blood flow is disturbed (non-uniform laminar flow, reversed or stationary flow, or oscillatory flow) [23, 24]. At these atherosclerosis-prone sites, the arterial wall is changing with plaque initiation and progression. Endothelial cells at these atherosclerotic-prone sites are activated and become more permeable for macromolecules such as low density lipoprotein (LDL) [24]. Besides increased cell permeability [24], activated endothelial cells are also characterized by diminished nitric oxide (NO) production [25]. NO is important for many functional processes in the arterial vessel wall [25-27] and alterations in NO-production are linked to various diseases such as atherosclerosis [27-29]. In addition, the endothelial glycocalyx may be locally thinned at sites of atherosclerosis, which may contribute to the increased permeability of the arterial wall and adhesion of monocytes [10-12, 30]. These monocytes adhere to the endothelial surface (fig. 2a), transmigrate into the subendothelial intima (fig. 2b) where they proliferate and differentiate into macrophages and take up lipoproteins, forming foam cells [4, 20-22]. These early lesions are called fatty streaks (fig. 2 b, c) [2].

Fatty streaks are prevalent in young people but are in general not yet symptomatic. With time, fatty streaks may develop into (advanced) atherosclerotic lesions which are symptomatic (atheroma). The accumulated foam cells die and a necrotic core develops inside the lesion, containing 
extracellular lipids (fig. 2d) [4]. Moreover, the initial inflammatory process is further expanded by innate and adaptive immunity responses [31], attracting more and more immune cells and vascular smooth muscle cells from the tunica media which further enhance lesion growth. The attracted and accumulated vascular smooth muscle cells secrete extracellular compounds, forming a fibrous cap that overlies the atherosclerotic lesion [3]. However, as a consequence of the inflammatory process, also enzymes such as matrixmetalloproteinases, collagenases, and elastases are produced that are known to breakdown the extracellular matrix, thereby weakening the fibrous cap [5, $20,32-34]$. With time, the fibrous cap of advanced atheromas gradually becomes thinner (and weaker) at the plaque shoulders, and ultimately the fibrous cap ruptures and the strongly thrombotic content of the necrotic core is left uncovered for the blood (fig. 2e) [3, 21, 34, 35]. The latter may result in thrombus formation which can occlude the artery, often causing acute ischemia in downstream organs, which may result in loss of function, and ultimately in death [3].

\section{Animal models of atherosclerosis}

Until recently, atherosclerotic research was mainly performed in animal models of cholesterol-fed rabbits, pigs, and non-human primates [36, 37]. Nowadays, the majority of atherosclerotic research is performed in mice (for extensive reviews see [36-39]). Unlike humans, normal (healthy) mice do not develop spontaneous atherosclerosis [37]. For that reason, mice were genetically altered, which resulted in various mouse models that do develop atherosclerosis within weeks to months after birth (dependent on type of model and type of diet). Most of these genetic mouse models lack important genes in the lipid metabolism, such as the low density lipoprotein receptor ( $\mathrm{LDLr}^{\circ}$ ) or apolipoprotein-E (ApoE ${ }^{-/}$) knock out mice. Lack of such genes leads to severe hypercholesterolemia and results in atherosclerotic plaque formation.

Plaque formation in these hyperlipidemic mouse models is comparable with the human atherogenesis [39, 40]. These mice develop human-like advanced atherosclerotic lesions with similar cellular composition and at similar sites in the vasculature. However, there are also differences. Mice have a different arterial geometry and lipid profile in the blood [41]. Furthermore, mouse models for spontaneous plaque rupture or the end-stage of atherosclerosis in humans, erosion of plaques, are still not available [36, 39]. There is an ongoing discussion concerning the interpretation and exact definition of plaque rupture in mice [42-44]. Due to the differences described above, extrapolation of mouse data to the human atherosclerosis should be conducted with care.

All studies presented in this thesis (except for chapter 6) were conducted in wild 
type (control) and atherosclerosis-prone ApoE $\%$ mice, in order to study structure and function of the healthy and atherosclerotic vascular wall of large arteries. Although sometimes the preparation procedures were technically challenging, the small size of mice is beneficial for visualization of the relatively thin wall of large arteries with Two-Photon Laser Scanning Microscopy (TPLSM).

\section{Imaging of large (diseased) arteries}

Imaging techniques such as magnetic resonance imaging, computer tomography, positron emission spectroscopy, or ultrasound, enable noninvasive visualization of various functional and structural aspects of living tissues, and have already contributed to the present knowledge of atherosclerosis $[45,46]$. However, the spatial resolution of these imaging modalities does not allow visualization of subcellular structures. Additionally, they lack the specificity that is required for visualization of delicate molecular properties of the (diseased) vasculature. These requirements are met by optical fluorescence microscopy which combines subcellular resolution with an exquisite molecular selectivity and sensitivity. In optical fluorescence microscopy, two basic principles of fluorescence excitation can be distinguished: single photon excitation and two-photon or multi-photon excitation. Both will be discussed in the next two paragraphs.

\section{Single-photon fluorescence microscopy}

Nowadays, conventional fluorescence microscopy is the most common mode of optical light microscopy used in biomedical research because of its great specificity and ease of use [47]. In fact, the already comprehensive knowledge of structure and function of the vasculature and vascular diseases such as atherosclerosis is in a large part based on studies utilizing conventional fluorescence microscopic techniques.

Both conventional fluorescence microscopy and confocal laser scanning microscopy are based on the principle of single-photon excitation (fig. 3). A fluorescent molecule (fluorophore) in the sample is excited by absorption of a single photon with a specific wavelength (between 350-650 nm). As a consequence of the subsequent decay of the molecule from the excited state to its ground state, fluorescent light of a slightly lower energy (i.e., longer wavelength; the difference between both wavelengths is called the Stokes shift) is emitted, allowing high contrast imaging of the distribution of fluorophores in the sample.

The process of fluorescence excitation occurs throughout the whole volume that is illuminated by the excitation light. Therefore, out-of-focus fluorescence light also contributes to image formation (fig. 4), which reduces contrast and signal- 
to-noise ratio, especially in thick and scattering tissues. Furthermore, bleaching of the fluorescent molecules not only occurs in the focal plane, but the entire volume illuminated. In combination with the high flux of excitation light that may cause cytotoxic effects, tissue viability is hampered in the large (illuminated) volume of the sample. Finally, single-photon (conventional) fluorescence microscopy lacks sufficient penetration depth to obtain highresolution images beyond the tissue surface due to the high absorption of the excitation light and the scattering properties of the emission light [47].

Confocal Laser Scanning Microscopy (CLSM) images are generated by pointto-point scanning through the focal plane.In CLSM, pinhole(s) are applied to block the out-of-focus light originating from the (single-photon) excited volume outside the focal plane [47-49]. Consequently, only light emitted in the focal plane contributes to the final image by largely removing the out-of-focus blur. CLSM allows optical sectioning and imaging inside the sample volume, beyond the tissue surface (up to $50 \mu \mathrm{m}$ ) [48, 49] and enables 3D image reconstruction $[47,48]$. However, CLSM still suffers from the lack of penetration depth in (scattering) tissue due to the excitation wavelengths used [47, 49, 50]. Also, with focus deeper in the tissue, scattered out-of-focus fluorescence may enter the pinhole thereby reducing contrast and resolution. Moreover, since the pinhole blocks a large part of the emitted light, imaging in the sample requires increased intensity of the excitation light (i.e. lasers of $5-50 \mathrm{~mW}$ [47]), Consequently, in thicker scattering tissues CLSM is strongly hampered by increased out-of-focus bleaching and cytotoxic effects (which still occurs throughout the whole depth of the sample) [51-53].

Microscopic techniques based on single-photon excitation are very useful for visualization in thin histological samples of large arteries. Preparation of such histological samples, i.e., fixating or freeze thawing and slicing, results in loss of viability and loss of functionality, and may lead to structural alterations [54, 55]. As a result, (3D) visualization of the elementary molecular processes in intact and viable atherosclerosis-prone arteries is compromised with these conventional microscopic techniques.

In order to elucidate the molecular processes that underlie the behavior of vascular cells and structures in their natural environment, and to translate the results obtained from cell cultures and histology to the situation that exists in viable intact samples ex vivo (see chapter 3-5) and ultimately in vivo (see chapter 7), a microscopic technique is required that does enable subcellular imaging and is capable of penetrating deep into viable, highly scattering blood vessels. 


\section{Two-photon (multi-photon) laser scanning microscopy}

In the early nineties, two-photon laser scanning microscopy (TPLSM) was developed by the group of Webb and colleagues [56]. Two-photon laser scanning microscopy (TPLSM) is based on the principle of two-photon excitation of fluorescent molecules which already was described in 1931 by Maria Göppert-Mayer [57, 58]. Fluorescent molecules are excited in a single quantitized event by nearly simultaneous absorption of two photons [56] [5962] (Fig. 3). The process of two-photon excitation only occurs when these two photons reach the fluorophore within attoseconds $\left(10^{-18} \mathrm{~s}\right)$ of each other. Moreover, since the energy of photons is inversely proportional to their wavelength, the photons should have approximately twice the wavelength (near infrared $\approx>800 \mathrm{~nm}$ ) as is required for single photon excitation (bluegreen $\approx 350-650 \mathrm{~nm}$ ) [59]. The resulting emission depends on the square of the excitation intensity $[51,56,59,61,63]$. Furthermore, since the probability of the two-photon excitation process is very low, the required local photon density must be very high; approximately $10^{6}$ times higher than that required for single-photon excitation [59]. As a consequence, very high (excitation) laser powers are required in order to yield detectable (two-photon excited) fluorescence.

Such high excitation powers are achieved with mode-locked, pulsed lasers which operate with only a small set of modes. The phase between these modes is locked so that there is destructive interference of the waves between propagating frequencies everywhere in the laser cavity, except at the point where waves add constructively [64]. The latter results in an ultra short $110^{-15}$ $\left.10^{-12} \mathrm{~s}\right)$, single pulse of light travelling in the cavity with a repetition rate ( $\approx 82 \mathrm{MHz})$ that is determined by the distance between the end mirrors of the cavity [65]. Mode-locked lasers are tuneable in the near infrared wavelength range $(700-900 \mathrm{~nm})$. In the TPLSM used in the studies described in this thesis, the energy of the laser light is concentrated in a pulse with a width of approximately $140 \mathrm{fs}$ at a wavelength of $790 \mathrm{~nm}$ (fig. 5). The resulting high peak power enables sufficient two-photon excitation while the average power is relatively low, which is beneficial for preservation of the biological sample. In TPLSM, the optimum conditions for two-photon excitation only exist in the very narrow focal volume of the microscope. As a result, fluorescence emission only occurs in this narrow focal volume (fig. 4), which results in very good optical sectioning and good signal-to-noise-ratio without the application of pinholes as required in CLSM. Since fluorescence emission is limited to the focal volume, detectable fluorescent (emission) light must originate from this focal volume, even though it might be scattered on its way to the detector. The use of longer wavelengths (reduced scattering and absorption) and the 
absence of out-of-focus absorption results in an increase in penetration depth. The maximal depth for good image quality achieved with TPLSM in arteries is over $100 \mu \mathrm{m}$, while the maximum depth with CLSM is limited to $50 \mu \mathrm{m}$ [50]). The absence of out-of-focus excitation, and the longer wavelength of the nearinfrared excitation light further reduce photo-damage, photo-toxicity, and photo-bleaching $[53,60,66,67]$. As in CLSM, image composition of TPLSM is based on point-to-point scanning through the optical section.

The characteristics of TPLSM potentially enable imaging of thick, scattering samples without compromising viability, and make it superior over singlephoton excitation microscopy such as $\operatorname{CLSM}[50,62,68-70]$.

In practice, TPLSM has further advantages over conventional fluorescence microscopy. Since only one excitation wavelength is required to excite multiple fluorophores, the requirements of blocking filters for the excitation light are reduced [67]. This simplifies multichannel imaging (i.e., simultaneous visualization of two or more fluorophores) and provides a broad uninterrupted emission collection bandwidth [65]. The latter may result in increased contrast (increased signal-to-noise-ratio). Due to the increased difference between the wavelength of the excitation laser and the emitted light, leak-through of the excitation light into the wavelength range (reducing signal-to-noise-ratio) of the emitted fluorescence is strongly reduced [67]. Since photo-damage, phototoxicity, photo-activation, and photo-bleaching are reduced, imaging times can be extended without compromising the viability of the sample, which may result in better quality of the obtained images.

However, TPLSM has also some drawbacks. (1) TPLSM is an expensive technique [59] and not yet routinely used for biological studies since it requires specific expertise for effective application [59, 65]. (2) Due to the longer wavelengths used for excitation light [59], the spatial resolution in TPLSM is somewhat reduced when compared with CLSM. However, this only holds for imaging at shallow depths in the sample. Indeed, the size of the excited focal volume in TPLSM does increase with increasing depths (due to scattering and the consequent increase of the excitation light intensity), but the resolution of TPLSM deteriorates far less with increasing depth than that of CLSM [50]. (3) Although photo-damage and photo-toxic effects are reduced, TPLSM can have negative consequences for the viability of biological samples [71, 72]. Twophoton excitation may cause heating of the sample, impaired cellular reproduction, oxidative stress, or apoptosis-like cell death. In order to avoid these effects, non-destructive imaging of live samples is limited to an optical window, dependent on the applied fluorophores, laser intensity, detector 
sensitivity, and the sample [71]. (4) TPLSM does not allow image acquisition at video rate $(20-100 \mathrm{~Hz}$ ) since image composition is based on the point-point scanning instead of collecting the full field of view at once (as in conventional fluorescence microscopy). This complicates (in vivo) imaging of motional structures or cells.

\section{Fluorescence probes to identify structures, cells and functionality}

Fluorescent probes suitable for structural imaging in viable thick arteries with TPLSM must meet at least 4 requirements.

(1) The applied fluorophore (either alone or coupled to a specific antibody or peptide) must be two-photon excitable. Theoretically, all fluorophores that can be excited with single photon excitation should be excitable by two-photons of each half the energy and thus double the wavelength. However, the probability of two-photon absorption of the fluorophore (two-photon action cross section) can differ from this simple assumption, resulting in an optimal two-photon excitation wavelength that strongly deviates from the normally applied excitation wavelength (between $750-900 \mathrm{~nm}$ ) [65, 70]. Although the optimum wavelength of such fluorophores might be available by tuning the excitation laser, this severely hampers the use of such fluorophores in combination with other fluorophores with a different optimal two-photon excitation wavelength. In practice, characterization of the fluorophore prior to application may give insight in the practicability of the probe alone or in combination with other probes. Many fluorophores have a strong enough emission to be excited at a non-optimal wavelength.

(2) The fluorescent probe is assumed to specifically home to the imaging target under consideration. Some of the available fluorescent probes home directly to a specific vascular or cellular target. In other cases, to achieve specificity of the probe, the fluorescent molecule has to be linked to an antibody or peptide tailor-made to be specific for the imaging target.

(3) In either case, the complete particle must be able to penetrate deep into the intact and mostly viable tissue in order to reach all structures within the sample. In that respect peptides often are preferable above antibodies, due to their smaller size. The permeability of the endothelium or internal elastic lamina in various vascular beds or organs is often an important determinant for the penetration of various fluorescent probes (chapter 3-5). Note that sometimes lack of permeability can be used advantageously to discriminate healthy from diseased tissue (chapter 4, 5). This property may be used to target structures of cells exclusively within the atherosclerotic lesion by application of specific contrast enhancing agents. This may enable plaque detection or molecular imaging (chapter 4, 5). 
(4) For intracellular targeting, an additional requirement is that the vehicle must be able to penetrate into viable cells with an intact and fully functional cell membrane (unlike fixed cells that have a compromised membrane).

For functional experiments some additional properties are required. Of course the probe must be specific, sensitive, non-toxic, and should not influence functionality. Furthermore a significant change in fluorescent properties le.g., spectral, intensity) must arise upon changes in functional characteristics. A color shift of the probe instead of an increase in intensity of the emitted fluorescence is preferred, since this is independent of external factors such as laser intensity (chapter 6).

In this thesis we have characterized novel probes for the detection of the extracellular matrix protein collagen and nitric oxide (NO) production. Collagen in the wall of blood vessels can be directly visualized using its second harmonic generation (SHG), a non-linear scattering process resulting from the TPLSM pulsed laser exitation [50, 73-75]. However, detection of SHG in large arteries is rather difficult and only applicable for visualization of collagen in the tunica adventitia (chapter 3). In order to visualize collagen in all tunicae of the arterial wall, a novel collagen probe [76] was evaluated and utilized in (diseased) large arteries (chapter 4, 5).

$\mathrm{NO}$ production is a functional and very dynamic process. In the vasculature, $\mathrm{NO}$ is an important endothelium-derived vasodilator that has been implicated with various diseases such as atherosclerosis [27-29]. Most currently available $\mathrm{NO}$-probes shift from non-fluorescent to fluorescent in the presence of downstream derivatives of $\mathrm{NO}$. Consequently, these probes often lack specificity for NO. Therefore, the properties of a relatively new NO-probe [77] that displays a spectral shift in the presence of $\mathrm{NO}$ were evaluated (chapter 6 ).

\section{Current status of two-photon microscopy of large arteries}

In recent years, TPLSM has already been employed in various studies in intact and viable tissues ex vivo and in relatively immobile tissues in vivo such as brain [78-80], kidney [70, 81-84], lymphatics [85-88], and tumors [89]. Up till now, TPLSM was only rarely used in studies of large arteries, although these vessels are eminently involved in several cardiovascular diseases. In all these studies, large arteries were isolated and imaged under non-physiological circumstances (i.e. non-viable [74, 90], sliced or cut open [91, 92], in the absence of pressure and longitudinal tension and with shortened longitudinal length $[50,93])$. The method of arterial preparation in these studies often resulted in total loss of functionality and structural deformations of the arterial 
wall such as the typical wave-like appearance of the internal elastic laminae, which is caused by longitudinal shortening and lack of transmural pressure [92]. Consequently, the preparation procedures in these studies do not allow simultaneous imaging of structural and functional properties of the arterial vessel wall.

Perfusion chamber in which arteries are cannulated and mounted on thin micropipettes, are routinely used for perfusion myography studies [94, 95]. This mounting procedure can also be applied for TPLSM imaging and has several advantages over imaging of non-mounted arteries (chapter 3). (1) Structural deformations of the vascular wall of isolated arteries as occurred in all previous TPLSM studies in large arteries, can be avoided by application of transmural pressure and correction of longitudinal shortening of the artery [ 6 , $92,96]$. (2) The viability of arteries mounted in perfusion chambers is maintained for several hours and (3) combined with easy application of fluorescent markers luminally and abluminally, it may allow combined structural and functional imaging under physiological circumstances. (4) Application of flow is possible, potentially enabling visualization of functional-structural remodeling processes relating to flow [97]. The present thesis presents results of TPLSM imaging obtained in isolated (diseased) large arteries that were cannulated and mounted in perfusion chambers (chapter 3-5) [98, 99].

The ultimate goal in vascular imaging is visualization of large arteries in vivo, at a subcellular level. Van Zandvoort and colleagues showed the feasibility of in vivo TPLSM imaging of large arteries [50]. However, images were severely hampered by strong motional disturbances that resulted from cardiac and respiratory activity. Triggering of the image acquisition on the cardiac- and respiration cycles (combined with accelerated acquisition speeds) may result in a reduction of the motional artifacts. First results of triggered in vivo TPLSM imaging of large arteries are presented in chapter 7 . 


\section{Figures}

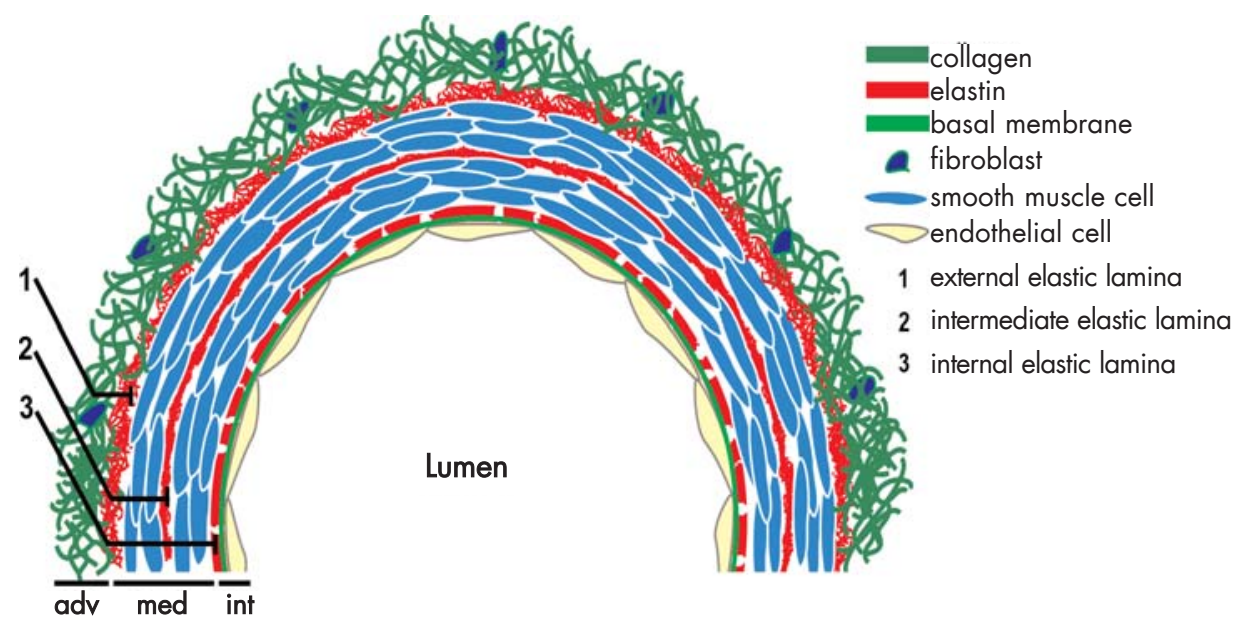

Figure 1

Simplified model of an arterial vessel wall. The number of vascular smooth muscle cell layers and intermediate elastic laminae is dependent on type of artery and species. Note the fenestrations (white) in the internal elastic layer.

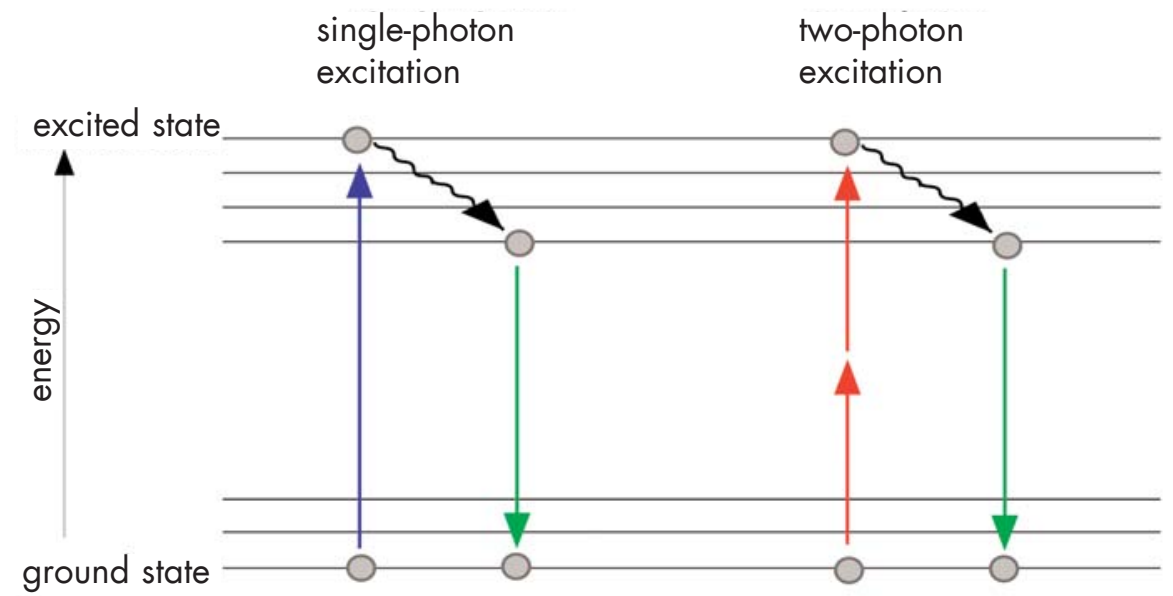

\section{Figure 3}

Simplified Jablonski energy diagrams of fluorescent molecules presenting single photon excitation (left), and two-photon excitation (right). 


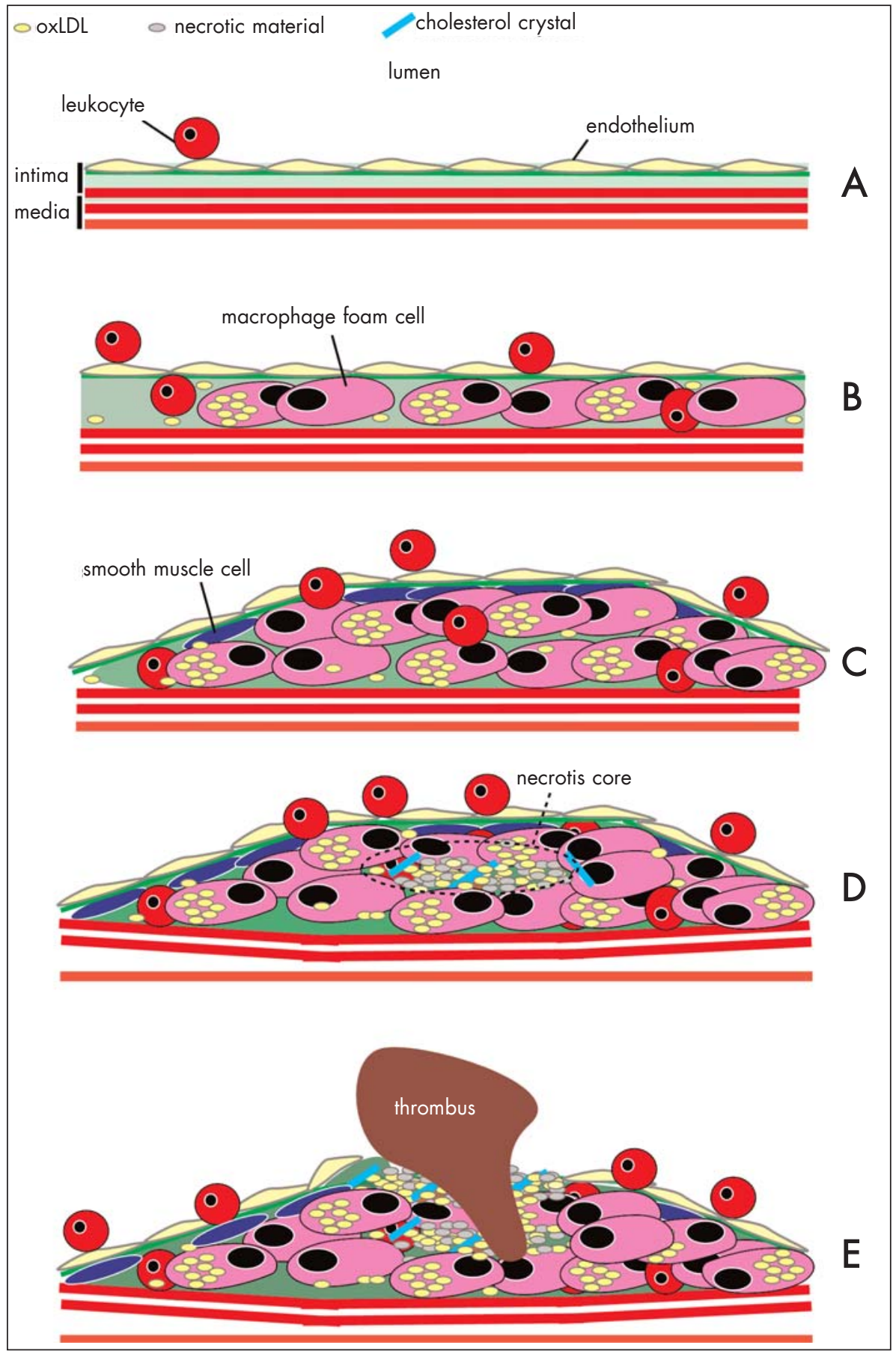

Figure 2

Impression of the most important features and cell types involved in atherosclerotic lesion development from the initial phase $(A)$ towards plaque rupture $(E)$. 


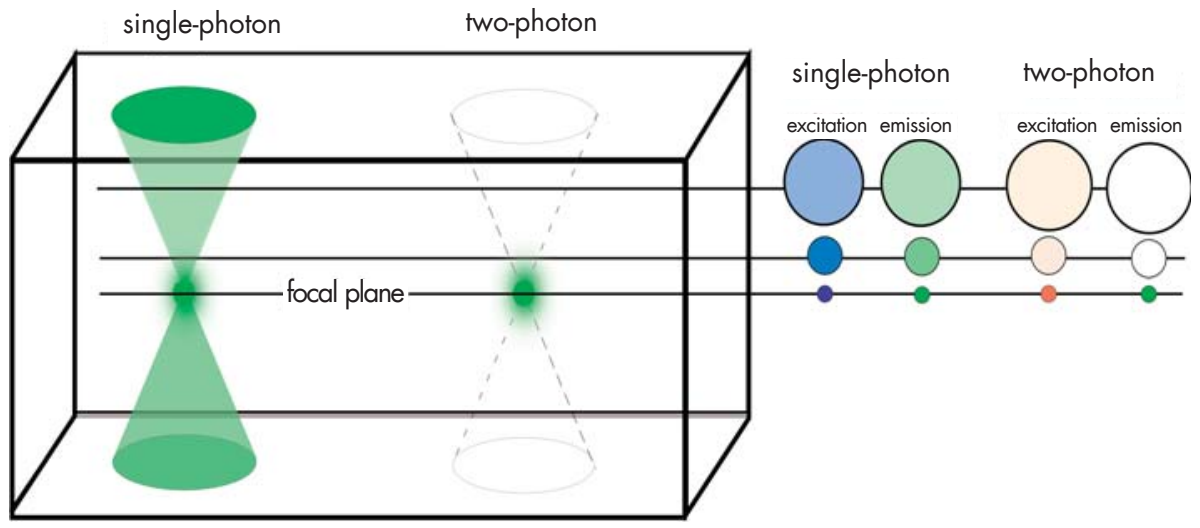

Figure 4

Single photon versus two-photon excitation; with single photon excitation fluorophores are excited throughout the complete beam path, resulting in emission above and below the focal spot. With two-photon excitation, fluorescence emission is restricted to the focal spot.

\section{continuous laser}

\section{Pulsed laser}

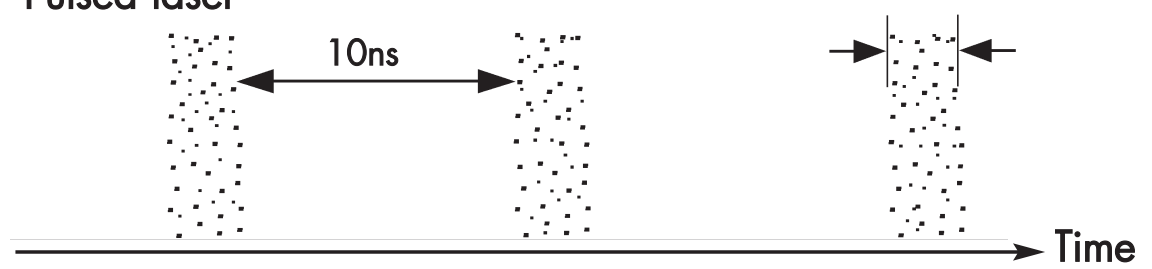

Figure 5

Continuous versus pulsed excitation lasers. Temporal compression of photons into short pulses achieves a high enough local density of photon to enhance the probability of two-photon absorption process, allowing two-photon excitation. The average power of such pulsed laser is comparable with a continuous laser, as used for single photon excitation. 


\section{References}

1. Stary, H., D. Blankenhorn, A. Chandler, et al., A definition of the intima of human arteries and of its atherosclerosis- prone regions. A report from the Committee on Vascular Lesions of the Council on Arteriosclerosis, American Heart Association. Circulation, 1992;85(1):391-405.

2. Stary, H.C., A.B. Chandler, S. Glagov, et al., A definition of initial, fatty streak, and intermediate lesions of atherosclerosis. A report from the Committee on Vascular Lesions of the Council on Arteriosclerosis, American Heart Association. Circulation, 1994;89(5):2462-78.

3. Stary, H.C., A.B. Chandler, R.E. Dinsmore, et al., A definition of advanced types of atherosclerotic lesions and a histological classification of atherosclerosis. A report from the Committee on Vascular Lesions of the Council on Arteriosclerosis, American Heart Association. Circulation, 1995;92(5):1355-74.

4. Lusis, A.J., Atherosclerosis. Nature, 2000;407(6801):233-41.

5. Ross, R., Atherosclerosis-an inflammatory disease. N Engl J Med, 1999;340(2):1 15-26.

6. Junqueira, L.C. and J. Carneiro, Basic Histology. eleventh edition ed. 2005, New York: McGraw-Hill Medical Publishing Division.

7. Cines, D.B., E.S. Pollak, C.A. Buck, et al., Endothelial cells in physiology and in the pathophysiology of vascular disorders. Blood, 1998;91(10):3527-61.

8. Ghitescu, L. and M. Robert, Diversity in unity: the biochemical composition of the endothelial cell surface varies between the vascular beds. Microsc Res Tech, 2002;57(5):381-9.

9. oude Egbrink, M.G., M.A. Van Gestel, M.A. Broeders, et al., Regulation of microvascular thromboembolism in vivo. Microcirculation, 2005;12(3):287-300.

10. Reitsma, S., D.W. Slaaf, H. Vink, et al., The endothelial glycocalyx: composition, functions, and visualization. Pflugers Arch, 2007;454(3):345-59.

11. Nieuwdorp, M., M.C. Meuwese, H. Vink, et al., The endothelial glycocalyx: a potential barrier between health and vascular disease. Curr Opin Lipidol, 2005;16(5):507-1 1.

12. Van Teeffelen, J.W., J. Brands, E.S. Stroes, et al., Endothelial glycocalyx: sweet shield of blood vessels. Trends Cardiovasc Med, 2007;17(3):101-5.

13. Dora, K.A., S.L. Sandow, N.T. Gallagher, et al., Myoendothelial Gap Junctions May Provide the Pathway for EDHF in Mouse Mesenteric Artery. J Vasc Res, 2003;40(5):480-490.

14. Sandow, S.L., K. Goto, N.M. Rummery, et al., Developmental changes in myoendothelial gap junction mediated vasodilator activity in the rat saphenous artery. J Physiol, 2004;556(Pt 3):875-86.

15. Sandow, S.L., M. Tare, H.A. Coleman, et al., Involvement of myoendothelial gap junctions in the actions of endothelium-derived hyperpolarizing factor. Circ Res, 2002;90(10):1 108-13.

16. Mahmoudi, M., N. Curzen, and P.J. Gallagher, Atherogenesis: the role of inflammation and infection. Histopathology, 2007;50(5):535-46.

17. Heistad, D.D. and M.L. Marcus, Role of vasa vasorum in nourishment of the aorta. Blood Vessels, 1979;16(5):225-38.

18. Gossl, M., M. Rosol, N.M. Malyar, et al., Functional anatomy and hemodynamic characteristics of vasa vasorum in the walls of porcine coronary arteries. Anat Rec A Discov 
Mol Cell Evol Biol, 2003;272(2):526-37.

19. Libby, P., Vascular biology of atherosclerosis: overview and state of the art. Am J Cardiol, 2003;91(3A):3A-6A.

20. Libby, P., P.M. Ridker, and A. Maseri, Inflammation and atherosclerosis. Circulation, 2002; 105(9): 1 135-43.

21. Libby, P., Changing concepts of atherogenesis. J Intern Med, 2000;247(3):349-58.

22. Hansson, G.K., Inflammation, atherosclerosis, and coronary artery disease. N Engl J Med, 2005;352(16):1685-95.

23. VanderLaan, P.A., C.A. Reardon, and G.S. Getz, Site Specificity of Atherosclerosis. SiteSelective Responses to Atherosclerotic Modulators. Arterioscler Thromb Vasc Biol, 2004;24(1):12-22.

24. Gimbrone, M.A., Jr., Vascular endothelium, hemodynamic forces, and atherogenesis. Am J Pathol, 1999;155(1):1-5.

25. Loscalzo, J. and J.A. Vita, Nitric Oxide and the Cardiovascular System. Contempory Cardiology, ed. C.P. Cannon. 2000, Totowa, New Jersey: Humana Press. 601.

26. Fleming, I. and R. Busse, Signal transduction of eNOS activation. Cardiovasc Res, 1999;43(3):532-41.

27. Shesely, E.G., N. Maeda, H.S. Kim, et al., Elevated blood pressures in mice lacking endothelial nitric oxide synthase. Proc Natl Acad Sci U S A, 1996;93(23):13176-81.

28. Dam, B.v., Nitric oxide and oxidative stress; focus on endthelial activation and diabetesinduced arteriolar changes, in Faculteit der Geneeskunde. 2003, Vrije Universiteit Amsterdam: Amsterdam. 158.

29. Ignarro, L.J., R.E. Byrns, G.M. Buga, et al., Endothelium-derived relaxing factor from pulmonary artery and vein possesses pharmacologic and chemical properties identical to those of nitric oxide radical. Circ Res, 1987;61(6):866-79.

30. van den Berg, B.M., J.A. Spaan, T.M. Rolf, et al., Atherogenic region and diet diminish glycocalyx dimension and increase intima-to-media ratios at murine carotid artery bifurcation. Am J Physiol Heart Circ Physiol, 2006;290(2):H915-20.

31. Hansson, G.K., P. Libby, U. Schonbeck, et al., Innate and adaptive immunity in the pathogenesis of atherosclerosis. Circ Res, 2002;91(4):281-91.

32. Lucas, A.D. and D.R. Greaves, Atherosclerosis: role of chemokines and macrophages. Expert Rev Mol Med, 2001;3:1-18.

33. Galis, Z.S., G.K. Sukhova, M.W. Lark, et al., Increased expression of matrix metalloproteinases and matrix degrading activity in vulnerable regions of human atherosclerotic plaques. J Clin Invest, 1994;94(6):2493-503.

34. Galis, Z.S. and J.J. Khatri, Matrix metalloproteinases in vascular remodeling and atherogenesis: the good, the bad, and the ugly. Circ Res, 2002;90(3):251-62.

35. Kolodgie, F.D., A.P. Burke, A. Farb, et al., The thin-cap fibroatheroma: a type of vulnerable plaque: the major precursor lesion to acute coronary syndromes. Curr Opin Cardiol, $2001 ; 16(5): 285-92$. 
36. Daugherty, A., Mouse models of atherosclerosis. Am J Med Sci, 2002;323(1):3-10.

37. Breslow, J.L., Mouse models of atherosclerosis. Science, 1996;272(5262):685-8.

38. Carmeliet, P., L. Moons, and D. Collen, Mouse models of angiogenesis, arterial stenosis, atherosclerosis and hemostasis. Cardiovasc Res, 1998;39(1):8-33.

39. Lutgens, E., R.J. van Suylen, B.C. Faber, et al., Atherosclerotic plaque rupture: local or systemic process? Arterioscler Thromb Vasc Biol, 2003;23(12):2123-30.

40. Lutgens, E., Cell Turnover and Immune Cell Activation: Key Factors in the Control of Plaque Progression and Phenotype in Atherosclerosis?, in Pathology. 2001, Maastricht University: Maastricht. 181.

41. Meir, K.S. and E. Leitersdorf, Atherosclerosis in the apolipoprotein-E-deficient mouse: a decade of progress. Arterioscler Thromb Vasc Biol, 2004;24(6):1006-14.

42. Hansson, G.K. and D.D. Heistad, Two views on plaque rupture. Arterioscler Thromb Vasc Biol, 2007;27(4):697.

43. Jackson, C.L., M.R. Bennett, E.A. Biessen, et al., Assessment of unstable atherosclerosis in mice. Arterioscler Thromb Vasc Biol, 2007;27(4):714-20.

44. Schwartz, S.M., Z.S. Galis, M.E. Rosenfeld, et al., Plaque rupture in humans and mice. Arterioscler Thromb Vasc Biol, 2007;27(4):705-13.

45. Tadrous, P.J., Methods for imaging the structure and function of living tissues and cells: 2. Fluorescence lifetime imaging. J Pathol, 2000;191(3):229-34.

46. Manka, D.R., W. Gilson, I. Sarembock, et al., Noninvasive in vivo magnetic resonance imaging of injury-induced neointima formation in the carotid artery of the apolipoprotein-E null mouse. J Magn Reson Imaging, 2000; 12(5):790-4.

47. Murphy, D.B., Fundamentals of light microscopy and electronic imaging. 1st edition ed. 2001, New York: Wiley-Liss Inc. 368.

48. White, J.G., W.B. Amos, and M. Fordham, An evaluation of confocal versus conventional imaging of biological structures by fluorescence light microscopy. J Cell Biol, 1987; 105(1):41-8.

49. Pawley, J., Handbook of biological confocal microscopy. Third edition ed, ed. J. Pawley. 2006, New York: Springer. 985.

50. van Zandvoort, M., W. Engels, K. Douma, et al., Two-photon microscopy for imaging of the (atherosclerotic) vascular wall: a proof of concept study. J Vasc Res, 2004;41(1):54-63.

51. Denk, W. and K. Svoboda, Photon upmanship: why multiphoton imaging is more than a gimmick. Neuron, 1997;18(3):351-7.

52. Iborra, F., P.R. Cook, and D.A. Jackson, Applying microscopy to the analysis of nuclear structure and function. Methods, 2003;29(2):131-41.

53. Sako, Y., A. Sekihata, Y. Yanagisawa, et al., Comparison of two-photon excitation laser scanning microscopy with UV-confocal laser scanning microscopy in three-dimensional calcium imaging using the fluorescence indicator Indo-1. J Microsc, 1997;185 ( Pf 1):9-20.

54. Bucher, D., M. Scholz, M. Stetter, et al., Correction methods for three-dimensional reconstructions from confocal images: I. Tissue shrinking and axial scaling. J Neurosci 
Methods, 2000;100(1-2):135-43.

55. Dorph-Petersen, K.A., J.R. Nyengaard, and H.J. Gundersen, Tissue shrinkage and unbiased stereological estimation of particle number and size. J Microsc, 2001;204(Pt 3):232-46.

56. Denk, W., J.H. Strickler, and W.W. Webb, Two-photon laser scanning fluorescence microscopy. Science, 1990;248(4951):73-6.

57. Göppert-Mayer, M., Über Elementarakte mit zwei Quantensprüngen. Ann. Phys., $1931 ; 9: 273-295$.

58. Masters, B.R. and P.T. So, Antecedents of two-photon excitation laser scanning microscopy. Microsc Res Tech, 2004;63(1):3-11.

59. Piston, D.W., Imaging living cells and tissues by two-photon excitation microscopy. Trends Cell Biol, 1999;9(2):66-9.

60. Xu, C., W. Zipfel, J.B. Shear, et al., Multiphoton fluorescence excitation: new spectral windows for biological nonlinear microscopy. Proc Natl Acad Sci USA, 1996; 93(20): 10763-8.

61. Diaspro, A. and M. Robello, Two-photon excitation of fluorescence for three-dimensional optical imaging of biological structures. J Photochem Photobiol B, 2000;55(1):1-8.

62. Rubart, M., Two-photon microscopy of cells and tissue. Circ Res, 2004;95(12):1154-66.

63. Diaspro, A., Introduction to two-photon microscopy. Microsc Res Tech, 1999;47(3): 163-4.

64. Hopkins, J.M. and W. Sibbett, Ultrashort-pulse lasers: big payoffs in a flash. Sci Am, 2000;283(3):72-9.

65. Zipfel, W.R., R.M. Williams, and W.W. Webb, Nonlinear magic: multiphoton microscopy in the biosciences. Nat Biotechnol, 2003;21(1 1):1369-77.

66. Gerritsen, H.C. and C.J. De Grauw, Imaging of optically thick specimen using two-photon excitation microscopy. Microsc Res Tech, 1999;47(3):206-9.

67. Bhawalkar, J., A. Shih, S. Pan, et al., Two-photon laser scanning fluorescence microscopy from a fluorophore and specimen perspective. bioimaging, 1996;4:168-178.

68. Williams, R.M., D.W. Piston, and W.W. Webb, Two-photon molecular excitation provides intrinsic 3-dimensional resolution for laser-based microscopy and microphotochemistry. Faseb J, 1994;8(1 1):804-13.

69. Squirrell, J.M., D.L. Wokosin, J.G. White, et al., Long-term two-photon fluorescence imaging of mammalian embryos without compromising viability. Nat Biotechnol, 1999;17(8):763-7.

70. Dunn, K.W. and P.A. Young, Principles of multiphoton microscopy. Nephron Exp Nephrol, 2006; 103(2):e33-40.

71. Konig, K., Multiphoton microscopy in life sciences. J Microsc, 2000;200 ( Pt 2):83-104.

72. Tauer, U., Advantages and risks of multiphoton microscopy in physiology. Exp Physiol, 2002;87(6):709-14.

73. Campagnola, P.J., A.C. Millard, M. Terasaki, et al., Three-dimensional high-resolution secondharmonic generation imaging of endogenous structural proteins in biological tissues. Biophys J, 2002;82(1 Pt 1):493-508.

74. Zoumi, A., X. Lu, G.S. Kassab, et al., Imaging coronary artery microstructure using second- 
harmonic and two-photon fluorescence microscopy. Biophys J, 2004;87(4):2778-86.

75. Zoumi, A., A. Yeh, and B.J. Tromberg, Imaging cells and extracellular matrix in vivo by using second-harmonic generation and two-photon excited fluorescence. Proc Natl Acad Sci USA, 2002;99(17): $11014-9$.

76. Krahn, K.N., C.V. Bouten, S. van Tuiil, et al., Fluorescently labeled collagen binding proteins allow specific visualization of collagen in tissues and live cell culture. Anal Biochem, 2006;350(2):177-85.

77. Meineke, P., U. Rauen, H. de Groot, et al., Nitric oxide detection and visualization in biological systems. Applications of the FNOCT method. Biol Chem, 2000;381 (7):575-82.

78. Svoboda, K., W. Denk, D. Kleinfeld, et al., In vivo dendritic calcium dynamics in neocortical pyramidal neurons. Nature, 1997;385(6612):161-5.

79. Helmchen, F. and J. Waters, Ca2+ imaging in the mammalian brain in vivo. Eur J Pharmacol, 2002;447(2-3): 119-29.

80. Levene, M.J., D.A. Dombeck, K.A. Kasischke, et al., In vivo multiphoton microscopy of deep brain tissue. J Neurophysiol, 2004;91 (4):1908-12.

81. Ashworth, S.L. and G.A. Tanner, Fluorescent labeling of renal cells in vivo. Nephron Physiol, 2006; 103(2):p91-6.

82. Dunn, K.W., R.M. Sandoval, K.J. Kelly, et al., Functional studies of the kidney of living animals using multicolor two-photon microscopy. Am J Physiol Cell Physiol, 2002;283(3):C905-16.

83. Yu, W., R.M. Sandoval, and B.A. Molitoris, Quantitative intravital microscopy using a Generalized Polarity concept for kidney studies. Am J Physiol Cell Physiol, 2005;289(5):C1 197-208.

84. Molitoris, B.A. and R.M. Sandoval, Intravital multiphoton microscopy of dynamic renal processes. Am J Physiol Renal Physiol, 2005;288(6):F1084-9.

85. Worbs, T., T.R. Mempel, J. Bolter, et al., CCR7 ligands stimulate the intranodal motility of $T$ lymphocytes in vivo. J Exp Med, 2007;204(3):489-95.

86. Zinselmeyer, B.H., J. Dempster, A.M. Gurney, et al., In situ characterization of CD4+ T cell behavior in mucosal and systemic lymphoid tissues during the induction of oral priming and tolerance. J Exp Med, 2005;201 (1 1):1815-23.

87. Mempel, T.R., S.E. Henrickson, and U.H. Von Andrian, T-cell priming by dendritic cells in lymph nodes occurs in three distinct phases. Nature, 2004;427(6970):154-9.

88. Mempel, T.R., M.L. Scimone, J.R. Mora, et al., In vivo imaging of leukocyte trafficking in blood vessels and tissues. Curr Opin Immunol, 2004;16(4):406-17.

89. Tozer, G.M., S.M. Ameer-Beg, J. Baker, et al., Intravital imaging of tumour vascular networks using multi-photon fluorescence microscopy. Adv Drug Deliv Rev, 2005;57(1):135-52.

90. Ferrara, D.E., D. Weiss, P.H. Carnell, et al., Quantitative 3D fluorescence technique for the analysis of en face preparations of arterial walls using quantum dot nanocrystals and twophoton excitation laser scanning microscopy. Am J Physiol Regul Integr Comp Physiol, 2006;290(1):R1 14-23.

91. Parasassi, T., W. Yu, D. Durbin, et al., Two-photon microscopy of aorta fibers shows 
proteolysis induced by LDL hydroperoxides. Free Radic Biol Med, 2000;28(1 1):1589-97.

92. Boulesteix, T., A.M. Pena, N. Pages, et al., Micrometer scale Ex Vivo multiphoton imaging of unstained arterial wall structure. Cytometry A, 2006;69(1):20-6.

93. Maffia, P., B.H. Zinselmeyer, A. Ialenti, et al., Images in cardiovascular medicine. Multiphoton microscopy for 3-dimensional imaging of lymphocyte recruitment into apolipoprotein-Edeficient mouse carotid artery. Circulation, 2007;115(11):e326-8.

94. Hilgers, R.H., S. Bergaya, P.M. Schiffers, et al., Uterine Artery Structural and Functional Changes During Pregnancy in Tissue Kallikrein-Deficient Mice. Arterioscler Thromb Vasc Biol, 2003;23(10): 1826-1832.

95. Bergaya, S., R.H. Hilgers, P. Meneton, et al., Flow-dependent dilation mediated by endogenous kinins requires angiotensin AT2 receptors. Circ Res, 2004;94(12):1623-9.

96. Dickhout, J.G. and R.M. Lee, Structural and functional analysis of small arteries from young spontaneously hypertensive rats. Hypertension, 1997;29(3):781-9.

97. De Mey, J.G., P.M. Schiffers, R.H. Hilgers, et al., Toward functional genomics of flow-induced outward remodeling of resistance arteries. Am J Physiol Heart Circ Physiol, 2005; 288(3):H1022-7.

98. Douma, K., R.T. Megens, S. Reitsma, et al., Two-photon lifetime imaging of fluorescent probes in intact blood vessels: A window to sub-cellular structural information and binding status. Microsc Res Tech, 2007.

99. Prinzen, L., R.J. Miserus, A. Dirksen, et al., Optical and magnetic resonance imaging of cell death and platelet activation using annexin a5-functionalized quantum dots. Nano Lett, 2007;7(1):93-100. 


\section{Chapter 3}

\section{Two-Photon Microscopy of Vital Murine Elastic and Muscular Arteries: Combined Structural and Functional Imaging with Subcellular Resolution.}

Journal of vascular research $2007 ; 44: 87-98$

R.T.A. Megens ${ }^{a}$; S. Reitsma ${ }^{\text {a }}$; P.H.M. Schiffers ${ }^{\text {b}}$; R.H.P. Hilgers ${ }^{\text {b }}$; J.G.R. De Mey ${ }^{\text {b; }}$ D.W. Slaaf ${ }^{a}$, ${ }^{\text {; }}$ M.G.A. oude Egbrink ${ }^{\text {; }}$; M.A.M.J. van Zandvoort ${ }^{a}$.

Departments of 'Biophysics, 'Pharmacology, and 'Physiology, Cardiovascular Research Institute Maastricht, Maastricht University, Maastricht, the Netherlands. 'Department of Biomedical Engineering, Eindhoven University of Technology, Eindhoven, the Netherlands. 


\section{Abstract}

Understanding vascular pathologies requires insight in structure and function, and, hence, an imaging technique combining subcellular resolution, large penetration depth, and optical sectioning. We evaluated applicability of twophoton microscopy (TPLSM) in large elastic and small muscular arteries under physiological conditions.

Elastic (carotid) and muscular (uterine, mesenteric) arteries of C57BL/6 mice were mounted in a perfusion chamber. TPLSM was used to assess viability of arteries and to visualize structural components elastin, collagen, nuclei, and endothelial glycocalyx. Functionality was determined using diameter changes in response to noradrenaline and acetylcholine.

Viability and functionality were maintained up to 4 hours, enabling assessment of structure-function relationships. Structural vessel wall components differed between elastic and muscular arteries: size (1.3 vs. $2.1 \mathrm{\mu m})$ and density (0.045 vs. 0.57 per $\left.(100 \mu \mathrm{m})^{2}\right)$ of internal elastic lamina fenestrae, smooth muscle cell density $\left(3.50\right.$ vs. 1.53 per $\left.(100 \mu \mathrm{m})^{3}\right)$, number of elastic laminae ( 3 vs. 2), and adventitial collagen structure (tortuous vs. straight). Endothelial glycocalyx in elastic arteries was $4.5 \mu \mathrm{m}$ thick, covering $66 \%$ of endothelial surface.

TPLSM enables visualization and quantification of subcellular structures in vital and functional elastic and muscular murine arteries, allowing unraveling of structure-function relationships in healthy and diseased arteries. 


\section{Introduction}

Alterations of structural and functional properties of the arterial wall may lead to vascular diseases such as atherosclerosis in the case of elastic arteries, and hypertension in the case of small resistance-sized arteries. The understanding of vascular diseases would benefit greatly when such structural and functional properties could be studied simultaneously. Functional studies (investigating diameter changes and force development during control and intervention) are routinely done in isolated vessels mounted on two pipettes in a perfusion chamber (perfusion myography) [1, 2] or in a wire myograph [3]. To combine structural data with functional information, an imaging technique is required which enables imaging in intact and viable vessels ex-vivo.

The combination of simultaneously performed structural and functional studies excludes (immuno) histology. Required is a microscopy method that allows for optical sectioning (to obtain 3D reconstructions of the tissue) and has a subcellular resolution and penetration depth (more than $100 \mu \mathrm{m})$. Confocal laser scanning microscopy (CLSM) offers optical sectioning and 3D reconstruction, but has a limited penetration depth $1<50 \mu \mathrm{m}$ in murine carotid artery) and its resolution deteriorates with depth [4]. Furthermore, photobleaching, photo-toxicity, and photo-damage caused by the (single photon) excitation light are substantial, which also limits the application of CLSM for functional imaging.

In 1990, two-photon laser-scanning microscopy (TPLSM) was developed by the group of Webb et al. $[5,6]$. TPLSM is based on the principle of two-photon excitation [6], where simultaneous absorption of two near-infrared photons (total energy is equivalent to that of one photon at half the wavelength) leads to the excited state of fluorescent molecules. The probability of two-photon absorption depends on the square of the intensity of the excitation light, and only takes place in a very narrow volume at the focal position of the microscope. Because out of focus absorption and fluorescence are absent, the detected emission light always originates from the focal position independent of scattering. Typical features of TPLSM are enhanced depth penetration (dependent on sample characteristics, up to $250 \mu \mathrm{m}$ in rat aortas [7]), good optical sectioning, and good resolution (maximum resolution approximately $0.3 \mu \mathrm{m}$ in $\mathrm{XY}$ and $0.9 \mu \mathrm{m}$ in $\mathrm{Z}$ direction, dependent on the wavelength of the excitation light [8]). In addition, photo-bleaching, photo-damage and phototoxicity are drastically reduced [8], enabling imaging of delicate structures in viable tissue. Recently, TPLSM was established as a valuable tool for imaging of blood vessels [4, 9-1 1], skeletal muscle arterioles [12], and microvasculature of the human uterus [13]. However, the blood vessels were very small [12], still 
treated with a fixative, freeze-thawed and sliced, or casted in hot $\left(>40^{\circ} \mathrm{C}\right.$ ) agarose gel, resulting in structural alterations and loss of functionality [14]. In the present study, our aim was to demonstrate that TPLSM is a useful tool for imaging of fresh intact arteries mounted in a perfusion chamber. Together with appropriate fluorescent markers, this should allow exploration of the actual vessel wall properties at a sub-cellular level in three dimensions. The utility of our method is illustrated with examples of both structural and functional imaging of cell nuclei, elastin layers and collagen. Elastin is a major component of the extracellular matrix. In the arterial wall, elastic fibers assemble to form elastic layers, important for the elasticity of arteries. Alterations in elastin structure and content are linked with vascular diseases, arterial remodeling, and vascular smooth muscle cell proliferation [15-17]. Another major substance of the extracellular matrix of arteries is collagen. Collagen is the fundamental component of basement membranes and is a major determinant for maintaining vascular integrity (tensile strength) [18]. The endothelial glycocalyx (EG), a very delicate layer covering endothelial cells, was studied in elastic arteries. Recently, interest in the EG has increased because of its possible role in hemodynamics [19], blood cell-vessel wall interactions [20, 21], and atherosclerosis [22].

Structural and functional integrity of mounted arteries was tested by assessing cell membrane integrity on the one hand and vasomotor responses to a vasodilating and a vasoconstricting substance on the other hand. Experiments were performed in both elastic arteries (carotid arteries) and muscular arteries (uterine arteries and first-order mesenteric artery side branches) of mice.

\section{Methods}

\section{Animals}

Experiments were approved by the local ethics committee on the use of laboratory animals. Procedures were in accordance with institutional guidelines. Carotid and first-order mesenteric arteries were derived from 12-20 weeks old C57BL/6J mice (Charles River, Maastricht, the Netherlands), that were anesthetized by subcutaneous administration of a mixture of xylazine (Xylazin $^{\circledR}$, Ceva Sante Animale BV, Naaldwijk, the Netherlands; 15 mg/kg body weigth b.w.) and ketamine (Nimatek ${ }^{\circledast}$, Eurovet, Cuijk, the Netherlands; $75 \mathrm{mg} / \mathrm{kg} \mathrm{b.w.).} \mathrm{After} \mathrm{euthanasia} \mathrm{the} \mathrm{carotid} \mathrm{arteries} \mathrm{and} \mathrm{the} \mathrm{mesentery} \mathrm{were}$ isolated. Uterine arteries of non-pregnant mice were isolated from uterine tissue obtained from female C57BL/6J mice (12-16 weeks of age, Charles River) after euthanasia by $\mathrm{CO}_{2}$. 


\section{Tissue preparation}

\section{Elastic arteries: carotid arteries}

Segments of common carotid arteries (length $\approx 6-8 \mathrm{~mm}$ ) were freed of adipose and connective tissue and carefully handled, only at their outer ends without stretching them, to keep them viable. To avoid contact with air, they were kept moist during the whole preparation procedure. Until further processing, arteries were stored (maximum 30 minutes) at $4{ }^{\circ} \mathrm{C}$ in Hanks Balanced Salt Solution (HBSS, pH 7.4), containing in $\mathrm{mM}$ : $\mathrm{NaCl} 144, \mathrm{HEPES} 14.9$, glucose 5.5, $\mathrm{KCl}$ 4.7, $\mathrm{CaCl}_{2} 2.5, \mathrm{KH}_{2} \mathrm{PO}_{4} 1.2$, and $\mathrm{MgSO}_{4}$ 1.2.

\section{Muscular arteries: first-order mesenteric arteries and uterine arteries}

After dissection, the mesentery or uterus was pinned out on a Petri dish (coated with silicon) and kept moist with HBSS. Adipose and connective tissue were carefully removed. One segment $(\approx 3 \mathrm{~mm})$ of a first-order mesenteric artery without side branches or one segment $(\approx 3 \mathrm{~mm})$ of the uterine artery at midpoint of the arterial arcade was isolated and stored in HBSS at $4{ }^{\circ} \mathrm{C}$.

\section{Mounting procedure}

After storage, each vessel was mounted in a home-built perfusion chamber (fig. $1 \mathrm{~A}$; IDEE BV, Maastricht, the Netherlands) filled with $10 \mathrm{ml} \mathrm{HBSS}\left(37^{\circ} \mathrm{C}\right)$. The artery was mounted on two glass micropipettes (tip diameters 120-150 $\mu \mathrm{m}$ for elastic arteries, 80-100 $\mu \mathrm{m}$ for muscular arteries) and residual luminal blood was carefully removed by gently flushing with HBSS. To correct for the shortening of the artery during isolation [23], a transmural pressure of $100 \mathrm{mmHg}$ was applied (using a modified Big Ben sphygmomanometer, Riester, Germany) and the distance between the two pipettes was adjusted until the mounted artery was straight. After this length adjustment, transmural pressure was set at $80 \mathrm{mmHg}$ in order to mimic physiological conditions [24]. All experiments were performed at $37{ }^{\circ} \mathrm{C}$ (Linkam scientific instruments MC60 heating stage, UK) in the absence of luminal flow. Imaging was performed in vessels at a transmural pressure of $80 \mathrm{mmHg}$ and was restricted to the central portion of the vessel segment.

\section{Staining procedures}

Nuclei of viable cells were labeled with the viable cell membrane permeable DNA / RNA markers SYTO41 ( $\left.\lambda_{\max \text { emission }}=480 \mathrm{~nm}\right)$ or SYTO13 $\left(\lambda_{\text {max emission }}=510 \mathrm{~nm}\right)$ (Molecular Probes, Leiden, the Netherlands). Propidium iodide (PI, $\lambda_{\text {max emission }}=620 \mathrm{~nm}$; Molecular Probes) was used to assess cell membrane integrity. Although elastin displays an autofluorescence signal [25], the autofluorescence is weak when compared to the fluorescence 
of added probes. Therefore, we have used eosin $\left(\lambda_{\text {max emission }}=545 \mathrm{~nm}\right.$; Molecular Probes) as a specific, strongly fluorescent elastin marker. Eosin allows us to visualize elastin in more detail and in combination with other dyes [4]. Collagen was visualized by second harmonic generation (a nonlinear scattering process which results in the emission of photons at exactly half the wavelength of the excitation light $\left(\lambda_{\text {excitation }}=840 \mathrm{~nm}\right)[4,25-27]$. The endothelial glycocalyx was visualized with fluorescein isothiocyanate labeled wheat germ agglutinin (WGA-FITC, $\lambda_{\text {max emission }}=515 \mathrm{~nm}$, Sigma-Aldrich, Zwijndrecht, the Netherlands) [28]. Wheat germ agglutinin is a lectin from Triticum Vulgaris, which targets $N$-acetylated glucosamine residues in hyaluronic acid and heparan sulfates. All fluorescent probes were dissolved in HBSS except for WGA-FITC which was dissolved in Phosphate Buffered Saline (PBS, pH 7.4) in order to prevent any interactions of the probe with glucose residues present in HBSS. Labeling was performed in the perfusion chamber and fluorescent probes were added 30 minutes prior to the start of image acquisition. Fluorescent labels were not washed from the preparation during measurements.

Final probe concentrations were: SYTO41 $1.5 \mu \mathrm{M}$, SYTO13 $1.5 \mu \mathrm{M}, \mathrm{PI} 1.5$ $\mu \mathrm{M}$, Eosin $0.25 \mu \mathrm{M}$, and WGA-FITC $2.6 \mu \mathrm{M}$. All probes were applied both intra- and extraluminally, with the exception of WGA-FITC that was perfused at $1 \mathrm{ml} / \mathrm{hr}$ (carotid arteries) or $0.3 \mathrm{ml} / \mathrm{hr}$ (mesenteric artery) for 30 minutes. When WGA-FITC is applied intra- and extraluminally, it also labels glycosaminoglycans in the tunica media and adventitia (unpublished data). To verify the specificity of WGA-FITC for the endothelial glycocalyx, arteries were flushed with hyaluronidase (2.6 $\mathrm{MM}$, type IV-S, Sigma Aldrich) for 60 minutes $\left(1 \mathrm{ml} / \mathrm{h}\right.$ at $37^{\circ} \mathrm{C}$ ) after WGA-FITC labeling. This enzyme is known to break down the glycocalyx in vivo [29].

\section{Viability / functionality of mounted arteries}

To illustrate the viability of arteries in our experimental setup, mounted carotid arteries were stained with a mixture of SYTO41 and PI. Images were recorded 15 minutes and 4 hours after the start of the incubation, in various segments of the common carotid artery. In between imaging, the mounted artery was kept at $80 \mathrm{mmHg}$ transmural pressure and $37^{\circ} \mathrm{C}$.

Functionality of the endothelial cells and smooth muscle cells of mounted muscular (mesenteric) arteries was evaluated by testing the acute vasomotor responses (change in luminal diameter) to the vasodilator acetylcholine (1 $\mu \mathrm{M}$, Sigma-Aldrich, Zwijndrecht, the Netherlands) during noradrenaline (1 $\mu$, Sigma-Aldrich) induced vasoconstriction [1]. Both were administered extraluminally. Luminal diameters were measured in yz-scans recorded prior to 
and during stimulation (after stabilization of the vessel diameter). All functionality experiments were performed in arteries that were stained with SYTO4 1 and PI.

\section{Imaging}

The perfusion chamber was positioned on a Nikon E600FN microscope (Nikon Corporation, Tokyo, Japan), coupled to a standard Biorad 2100MP multiphoton system (Biorad, Hemel Hempstead, GB). The excitation source was a 140 femtosecond pulsed Ti: sapphire laser (Spectra Physics Tsunami, Mountain View, USA) tuned and mode-locked at either $840 \mathrm{~nm}$ (to visualize collagen by second harmonic generation) or $800 \mathrm{~nm}$ (for visualization of fluorescent probes). Laser light reached the sample through the microscope objective $160 \mathrm{X}$ water dipping, numerical aperture 1.0, working distance 2 $\mathrm{mm} ; 40 \mathrm{X}$ water dipping, numerical aperture 0.8 , working distance $2 \mathrm{~mm}$ ). Maximum field of view was 206 * $206 \mu \mathrm{m}$ for the $60 \mathrm{X}$ objective and 309 * $309 \mu \mathrm{m}$ for the $40 \mathrm{X}$ objective. When necessary, further magnification land thus higher pixel resolution) was achieved by optical zoom in the scan head. Three photo multiplier tubes (PMTs) were used to detect the emitted fluorescent signals. For imaging of each of the fluorescent markers, PMTs were tuned corresponding to parts of the emission spectra of the fluorescent markers used: SYTO4 1, 470-480 nm (PMT 1); SYTO 13, 500-540 nm (PMT 2); WGA-FITC 500-550 nm (PMT 2); Eosin, 530-560 nm (PMT 2) and / or 560-590 nm (PMT 3); PI, 560-600 nm (PMT 3). Second harmonic generation signal was collected at $400-450 \mathrm{~nm}$ in PMT 1 for excitation at $840 \mathrm{~nm}$. For simultaneous imaging of a combination of fluorescent markers, each PMT was tuned for minimal bleed-through of the fluorescent markers used. To prevent photochemical and thermal damage to the arteries, laser power was kept as low as possible. Frame rate was $0.1 \mathrm{~Hz}$ with a pixel dwell time of $39 \mu \mathrm{s}$, or $0.3 \mathrm{~Hz}$ with a pixel dwell time of $12 \mu$ s combined with Kalman filtering ( $n=3$ cycles) for noise reduction. From each PMT, separate images of 512 * 512 pixels were obtained and combined into a single image. Images were recorded in the $x y-$ plane. Inaccurate alignment of the pipettes in the perfusion chamber usually caused imaging of the artery in a slightly oblique plane. Series of xy-images at successive depths (z-stack) were collected for reconstruction of 3D-images (fig. 1 B). Luminal diameters were obtained from yz-images. To obtain yz-images with square pixels, z-step distance was equal to the pixel dimensions in $x y-$ direction.

\section{Image analysis}

Image processing was performed using Image-Pro Plus 6.0 (Media Cybernetics 
Inc., Silver Spring, USA). The 3D-constructor 5. 1 software (Media Cybernetics) was used for 3D-reconstructions. Image analysis was performed by two independent observers.

Six elastic (carotid) arteries and six muscular (uterine) arteries were used for quantitative analyses of cell nuclei and elastin. Vascular smooth muscle cell (vSMC) density was expressed as mean number of cells per $(100 \mu \mathrm{m})^{3}$ vessel wall tissue. The overall vessel wall volume was estimated in each z-stack by application of an iso-surface on the 3D-reconstruction. Iso-surface rendering yields a smoothed surface around all voxels above a chosen threshold. The applied iso-surface was based on the signal of cell nuclei (SYTO41). This is a good measure for total vessel wall volume since it includes all structures from the endothelial cells in the tunica intima to fibroblasts, which are widespread in the tunica adventitia. However, it excludes endothelial glycocalyx volume. The iso-surface threshold was based on the averaged full width at half maximum value of three randomly selected line profiles (in the xz-plane) of each z-stack. The endothelial cell (EC) density is expressed as mean number of cells per $(100 \mu \mathrm{m})^{2}$ luminal surface area.

Glycocalyx thickness and glycocalyx coverage (ratio of glycocalyx covered surface area and total surface area) were assessed in five carotid arteries. Analyses were performed by quantification of the glycocalyx volume and luminal surface area (as described in the previous paragraph). Mean thickness of glycocalyx was calculated by dividing the glycocalyx volume by the glycocalyx coverage.

Vasomotor responses, i.e. functionality of arteries, were determined in three mesenteric arteries and measured as changes in luminal diameter in yz-scans of the vessel.

\section{Statistics}

Results are presented as mean \pm standard deviation and were tested for significance using the Mann-Whitney test (non-parametric test for two independent groups). A value of $p<0.05$ was considered to be statistically significant. Inter-observer reliability for cell counting was estimated by a "twoway mixed consistency intra-class correlation coefficient". All statistical analyses were performed using SPSS 13.0 software (SPSS inc. USA).

\section{Results}

\section{Characteristics of the method}

\section{Geometric deformations}

Fig. 2 displays images of a carotid artery stained for elastin (red) and nuclei (blue). Fig. 2 A-B shows geometric deformations caused by dissection and lack 
of transmural pressure. Dissection induced longitudinal shortening of the artery in the absence of transmural pressure and length adjustment. The three elastic laminae were difficult to identify, appeared to be inconsistent and partially collapsed. Vessel wall thickness was $57 \pm 6 \mu \mathrm{m}$. In contrast, when the artery was straightened and a transmural pressure of $80 \mathrm{mmHg}$ was applied in order to mimic physiological conditions (fig. 2 C-D), vessel wall thickness was reduced to $33 \pm 4 \mu \mathrm{m}$. Furthermore, the elastic laminae appeared to be homogeneous and unfolded.

\section{Viability/ vitality of vessels}

Mounted arteries $(n=3)$ were stained with SYTO4 1 and PI to assess the viability of arteries. Images were recorded 15 minutes and 4 hours after the start of the incubation, in various segments of the arteries (fig. 3). After 15 minutes, $31 \%$ of the cell nuclei in the adventitial layer were Pl-positive. Only $2 \%$ of the vSMCs was Pl-positive and no Pl-positive ECs were detectable. After 4 hours (fig. $3 \mathrm{~A}$ ), the number of PI-positive nuclei in the 3 tunicae was unaltered, which indicates that vital cells in the artery remained vital. Intraluminal air bubbles (fig. 3 B) caused a shift from PI-negative to Pl-positive labeling of $95 \%$ of ECs within 10 minutes. The number of Pl-positive vSMCs and nuclei located in the adventitia was unaltered by intraluminal air bubbles. In contrast, after a single pinch with a forceps, the number of Pl-positive smooth muscle cells and adventitial cells in the visualized part of the vessel increased to $33.3 \%$ and $47.5 \%$ respectively. Parts of the EC layer at the site of pinching were denuded. Residual ECs at the pinching site were all PI-positive.

The functionality of three mounted arteries was assessed by measuring the luminal diameter change in mesenteric arteries during noradrenalin constriction (fig. 4). The average luminal diameter prior to stimulation was $246 \pm 21 \mu \mathrm{m}$. During noradrenaline administration the average luminal diameter declined to $182 \pm 3 \mu \mathrm{m}$. Subsequent administration of acetylcholine induced a vasodilatation; luminal diameter increased to $231 \pm 6 \mu \mathrm{m}$.

\section{Structural imaging}

\section{Elastin}

Fig. 5 B-E shows $x y$-slices of an elastic (carotid) artery, recorded at 4 z-positions (fig. $5 \mathrm{~A}$ ) with $z=0 \mu \mathrm{m}$ being the outside of the artery. Fig. 6 B-E are $x y$-slices of a muscular (uterine) artery, recorded at comparable positions in the arterial wall (fig. 6 A). The external elastic lamina was clearly visible in both types of arteries. The structure of the external elastic layer in elastic carotid arteries was more compact (fig. $5 \mathrm{C}$ ), whereas in the muscular uterine arteries, a mesh-like structure was observed (fig. 6 C). Clearly visible in all carotid arteries under 
investigation, was the appearance of an intermediate elastic layer (red) in the tunica media (fig. 5 D). This third elastic layer was not present in the investigated muscular arteries (fig. 6 C, D). The internal elastic lamina (IEL) was clearly distinguishable in muscular (uterine and mesenteric) arteries (fig. 6 D) but less apparent in carotid arteries (fig. 5 D). Fenestrae, non-fluorescent cylindrical patches in the internal elastic lamina [15], were visible in the internal elastic lamina. Their mean diameter was significantly larger in muscular arteries (diameter of 2.1 $\pm 0.2 \mu \mathrm{m}$ ) compared to that in elastic arteries $(1.3 \pm 0.4 \mu \mathrm{m}$ in elastic arteries, $\mathrm{p}=0.02)$. Furthermore, the density of fenestrae was significantly higher $\left(57.4 \pm 9.9\right.$ per $(100 \mu \mathrm{m})^{2}$ in muscular vs. $4.5 \pm 3.9$ per $(100 \mu \mathrm{m})^{2}$ in elastic arteries, $\left.\mathrm{p}=0.02\right)$.

\section{Nuclei}

Cell nuclei in the arterial vessel wall were labeled with SYTO13 (fig. 5 and 6). Based on their morphology, orientation and position relative to the elastin bands, nuclei can either be distinguished as endothelial cell nuclei (EC) in the tunica intima, vascular smooth muscle cell nuclei (vSMC) in the tunica media, or as fibroblast-like cell nuclei in the tunica adventitia (FB) [30]. EC density was not significantly different between elastic and muscular arteries $(11.8 \pm 2.2$ in elastic vs. $12.4 \pm 3.8$ in muscular arteries, per $(100 \mu \mathrm{m})^{2}$ surface area). VSMC density appeared to be significantly higher in elastic arteries $1350 \pm 52$ per $\left.(100 \mu \mathrm{m})^{3}\right)$ than in muscular arteries $\left(153 \pm 31\right.$ per $\left.(100 \mu \mathrm{m})^{3}, \mathrm{p}=0.004\right)$. Intraclass correlation for inter-observer reliability was 0.997 .

\section{Collagen}

Collagen was visualized in elastic and muscular arteries (fig. 7) with second harmonic generation (SHG) using backwards propagated light [4]. Since SHG is dependent on the peak laser intensity [31], the SHG-signal in arteries weakened with increasing depth and was only visible in the tunica adventitia of both elastic and muscular arteries at relatively high laser power (>30 mW). The shape of the collagen fibers in mounted elastic arteries (which were corrected for geometrical deformations) appeared to be tortuous (fig. 7 A) whereas in similarly mounted muscular arteries (fig. 7 B) the collagen fibers appeared to be more stretched out. Furthermore, in muscular arteries the SHGsignal was weaker and required higher laser power (>60 mW).

\section{Endothelial glycocalyx}

The endothelial glycocalyx was visualized in both muscular (fig. 8 A) and elastic (fig. 8 B) arteries. Mean thickness of the glycocalyx in carotid arteries was $4.5 \pm 1.0 \mu \mathrm{m}$; mean glycocalyx coverage of the endothelium was $66 \pm 6 \%$. 
To verify the specificity of the WGA-FITC labeling, the glycocalyx layer in carotid arteries was degraded enzymatically by hyaluronidase perfusion. Hyaluronidase treatment reduced the glycocalyx coverage to $25 \pm 2 \%$ (fig. 8 C) but did not affect the number of Pl-positive (i.e. viable) endothelial cells.

\section{Discussion}

In this study we demonstrated that the combination of TPLSM and arteries mounted in a perfusion chamber is a valuable tool for simultaneous imaging of structure and function of elastic and muscular arteries. We have shown that TPLSM is a very suitable microscopic technique for detailed imaging of intact and vital arteries in three dimensions, mainly because of its combination of a large penetration depth with good spatial resolution and optical sectioning. Furthermore, it allows quantification of delicate structures in the vessel wall, as illustrated by the visualization of the endothelial glycocalyx through the vessel wall. Until now, this thin layer, situated on the luminal site of the tunica intima, was difficult to visualize. Mounting of arteries on micropipettes is an established method that allows studying of functional properties of arteries [1, 2]. When combined with TPLSM, it enabled detailed imaging of arterial structures in physiological conditions. Furthermore, we have shown that the combination of both techniques allows functional imaging of large arteries. Therefore, this experimental setup can contribute to unraveling structurefunction relationships in healthy and diseased arteries.

TPLSM imaging of vital and mounted arteries has a number of advantages over existing methods for arterial imaging. (1) The viability of mounted arteries is maintained. This was illustrated by a vasoconstriction-vasodilatation protocol resulting in changes in arterial diameter (visualized in xz-images). In addition, this demonstrates the feasibility of imaging structural-functional relationships of large arteries, similar to those already performed in skeletal muscle arterioles [12]. Staining and frequent TPLSM image acquisition of mounted arteries did not affect viability (up to 4 hours after mounting), which is essential for prolonged imaging of functional processes. (2) The ability to control transmural pressure and luminal flow in mounted arteries will also enable exploration of pressure or flow-mediated structure-function relationships of arteries with subcellular resolution. (3) Structural deformation due to isolation of arteries can be resolved. The wave-like appearance of the vessel wall, typical for unmounted arteries [10] and histological sections, disappeared when pressure was applied and arterial length was adjusted. Therefore, the data presented in our study are more representative for the in vivo situation. (4) It enables extraand intraluminal labeling, the latter being virtually impossible in non-mounted 
arteries, which improves structure-specific labeling and simplifies labeling of structures in the tunica intima. For example, WGA-FITC is only specific for the endothelial glycocalyx when administered intraluminally.

Despite all these advantages, TPLSM imaging of mounted arteries also has its limitations. Even though it's good theoretical spatial $(x y \approx 0.3 \mu \mathrm{m})$ and axial $(z \approx 0.9 \mu \mathrm{m})$ resolution, TPLSM suffers from image deterioration in the axial direction. Especially at larger depths structures appear somewhat blurred due to a relatively large point spread function (PSF). Therefore, deconvolution of image stacks may enhance contrast, axial resolution and the signal-to-noise ratio. However, it is difficult to determine the (true) PSF inside the vessel wall in large arteries (which is a prerequisite for appropriate deconvolution). Additionally, according to the Nyquist criterium, the necessary pixel resolution needed for appropriate deconvolution must approach the maximal resolution (1024* 1024 pixels, step size $\leq 0.3 \mu \mathrm{m})$. This maximal pixel resolution in TPLSM however, is often limited by practical causes. Imaging of large arteries at maximum pixel resolution is time-consuming, results in large data stacks which are difficult to handle (especially during image analysis and restoration), and increase radiation load to the tissue. In practice, a resolution of $x y \approx 0.6$ $\mu \mathrm{m}, \mathrm{z} \approx 1.5 \mu \mathrm{m}$ in large arteries is more realistic.

The present study shows that our method enables identification of biologically interesting variations between different vessel categories. We have visualized several structural differences between elastic (carotid) and muscular (uterine and mesenteric) arteries. In the large elastic arteries an extra elastic layer was present in the tunica media, the structure of the external elastic lamina was more compact and the internal elastic lamina (IEL) contained significantly fewer and smaller fenestrae. IEL fenestrae have been implicated in electrical coupling between the endothelium and the underlying vSMCs [32, 33]. One consequence of this coupling, endothelium-dependent vasodilatation through vSMC hyperpolarization, is more prominent in small muscular arteries than in large elastic arteries [34]. The vSMC density was significantly higher in elastic arteries. Apparently, vSMCs are smaller in elastic arteries. Endothelial cell density was comparable. Collagen fibers in the tunica adventitia of elastic arteries have an undulating pattern, even when corrected for geometric deformations, whereas in muscular arteries the fibers have a straight appearance. Collagen in the tunica adventitia was visualized using its second harmonic generation (SHG). However, we were unable to detect SHG of collagen in other parts of the vessel wall, in contrast to result by Zoumi and colleagues. They established the existence of SHG signal in the tunica media 
in transversal sections of porcine coronary arteries [25]. In our setup, lack of SHG signal in the tunica media and intima limits the applicability of SHG as a contrast agent for collagen. A newly developed collagen probe (CNA35/OG488) may be advantageous for studying collagen structures in the tunica media and tunica intima [35].

Existing methods to visualize and quantify the glycocalyx in blood vessels often depend on electron microscopy, which implies extensive tissue processing and sectioning $[22,36,37]$, and excludes combination with functional studies. In the microcirculation, the glycocalyx can be visualized in vivo using fluorescently labeled lectins, proteoglycan-binding proteins or specific antibodies [21, 38]. However, the intravital microscopic techniques used in these models cannot be used in larger arteries due to limited penetration of the excitation light. A combination of TPLSM imaging and mounting of arteries proves to be a good approach to visualize the endothelial glycocalyx in larger arteries in three dimensions. Since mounted arteries maintain their viability and functionality and can be kept under more physiological conditions, the observed glycocalyx is probably representative of the in vivo situation. The mean thickness of the endothelial glycocalyx in carotid arteries in this study $(4.5 \pm 1.0 \mu \mathrm{m})$ is comparable with observations described in literature $(2.6 \pm 0.5$ $\mu \mathrm{m}$, in mesenteric arteries with a luminal diameter of approximately $150 \mu \mathrm{m}$ ) [39]. Mean endothelial glycocalyx coverage of the endothelial cell layer in common carotid arteries is $66 \%$. This incomplete coverage of the endothelial cell layer by glycocalyx may be caused by several factors. First, WGA-FITC may only label part of the heterogeneously distributed components $[40,41]$ of the glycocalyx, causing areas of the glycocalyx to be unlabeled. Second, weakly stained areas of glycocalyx did not contribute to the total area when the glycocalyx also contained areas with a much stronger labeling. This is a consequence of the applied image analysis procedure based on the full width at half maximum of the intensity signal of WGA-FITC, averaged over the total endothelial area within each image stack. Third, although mounted arteries were handled with care and were still viable, it cannot be excluded that some areas of the very delicate glycocalyx were damaged due to the isolation (the lumen of arteries occasionally collapsed during isolation) and mounting procedure.

In conclusion, the described method for imaging of viable arteries is a significant step forward in the field of vascular research, potentially enabling investigation of structure-function relationships in healthy and diseased blood vessels. 


\section{Figures}
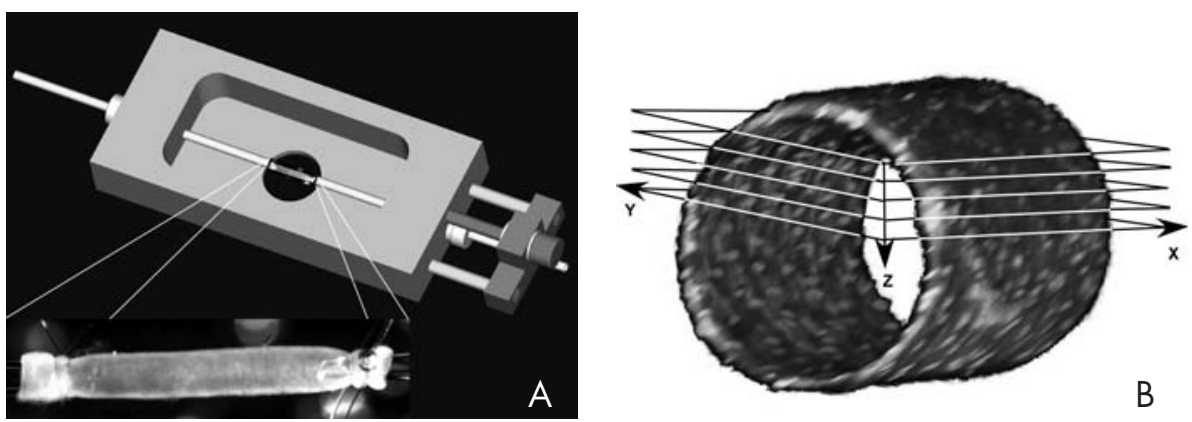

Figure 1

A: Schematic representation of a vessel perfusion chamber used to mount isolated arteries. The distance between the two pipettes is adjustable for correction of the arterial length after dissection. Fluid capacity is $10 \mathrm{ml}$. Inset: mouse uterine artery (external diameter $\approx 250 \mu \mathrm{m}$, length $\approx 3000$ $\mu \mathrm{m})$ mounted on two glass pipettes with sutures, length-adjusted, and pressurized to $80 \mathrm{mmHg}$. B: 3D-reconstruction (size of arterial section $=309 * 309 * 315 \mu \mathrm{m}$ ) of a uterine artery stained for elastin and nuclei with superimposed coordinate system and a z-stack of optical xy-slices.
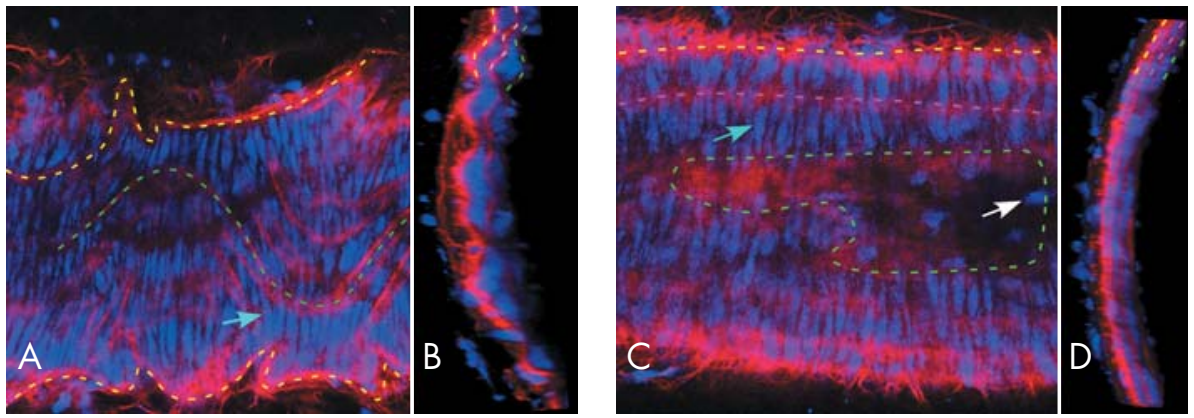

Figure 2

Carotid artery labeled for elastin (Eosin, red) and nuclei (SYTO41, blue) imaged at approximately the same z-position near transition of tunica media and tunica intima (A, C) and y-position (B, D). A and $B$ are obtained in the absence of transmural pressure and length adjustment. Elastin bands appear to be folded, and have a wave-like appearance. Furthermore, the internal elastic layer (green dashed line) and the intermediate elastic layer (pink dashed line in yz-reconstruction) and external elastic layer (yellow dashed lines) are difficult to distinguish from each other. VSMC nuclei (blue arrow) are visible in blue. The yz-reconstruction (B) clearly establishes the elastic laminae to be folded.

$C$ and $D$ display the same artery in the presence of transmural pressure $(80 \mathrm{mmHg})$ and length adjustment. All three elastic laminae (external elastic lamina, yellow dashed line; intermediate elastic lamina, pink dashed line; internal elastic lamina, green dashed line) are clearly recognizable and relatively straight. Smooth muscle cell nuclei (blue arrow) are homogeneously distributed and their (mean) orientation is perpendicular to the longitudinal axis of the artery. Furthermore, a few endothelial cell nuclei (pink arrow) are visible (due to a slightly oblique imaging plane). The yzreconstruction $(D)$, reconstructed from a stack of $x y$-sections, demonstrates that the vessel wall is tight and contains three elastic laminae. 

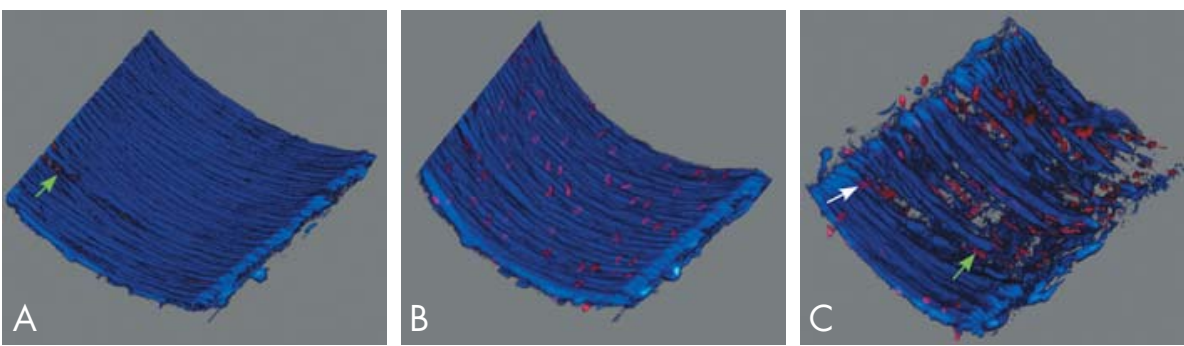

Figure 3

A: SYTO41 (vital, blue) and PI (cells with a compromised cell membrane, red) positive cells in 3D-reconstruction (approximate size $206 * 206 * 40 \mu \mathrm{m}$ ) of the central portion of a mounted mesenteric artery imaged 4 hours after start of the incubation. Only few Pl-positive smooth muscle cells are visible (green arrow). ECs are only faintly labeled with SYTO41 and, hence, hardly visible. After application of intraluminal air (B), most endothelial cell nuclei became PIpositive (red). A single pinch with a forceps (C) caused a local shift from SYTO41 to PI-positive nuclei of smooth muscle cells (green arrow) and endothelial cells (white arrow) within 10 minutes.
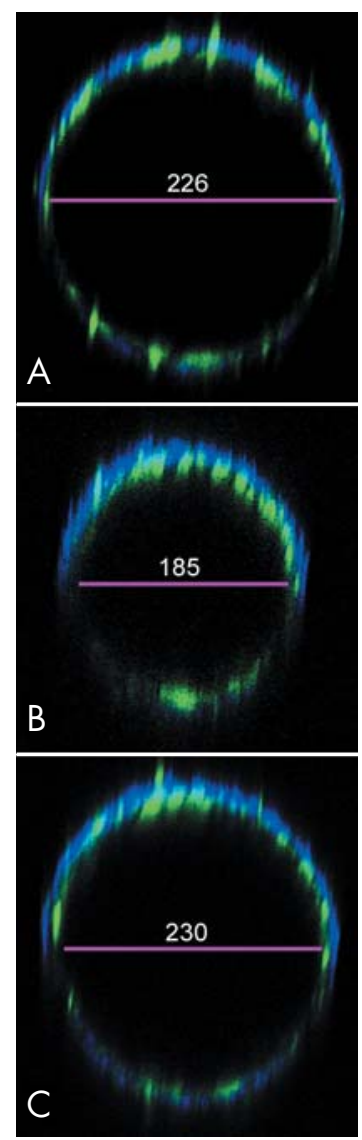

\section{Figure 4}

Typical yz-scans of a mesenteric artery in the abscence $(A)$ and presence of noradrenaline alone (B), and both noradrenaline and acetylcholine (C). Nuclei of cells were labeled with SYTO4 1 (pseudo-colored green), collagen was visualized with second harmonic generation (SHG, blue). A Gaussian filter was applied to reduce noise. Note that signal (especially the $\mathrm{SHG}$ ) is weakened at the lower parts of the yz-scans. 
$x$

A

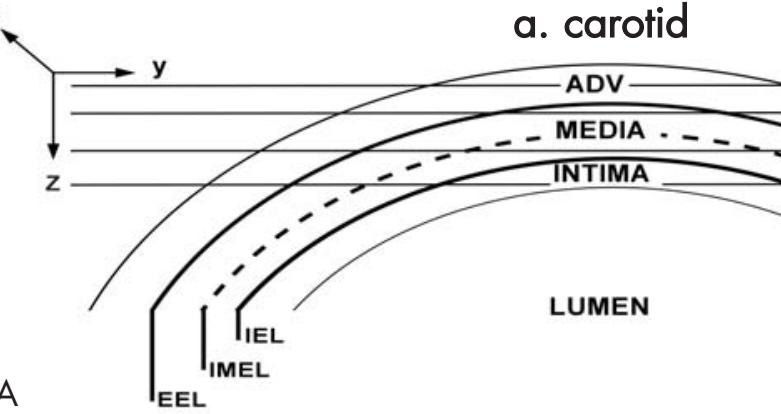

Figure 5
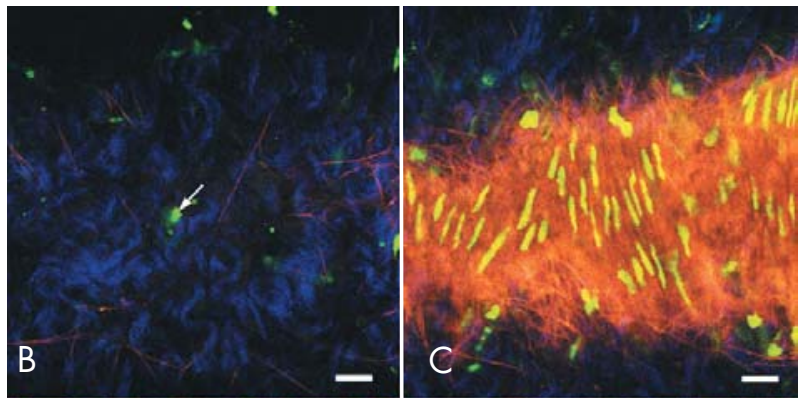

A: Schematic view of the arterial wall representing the positions of the $x y$-sections shown in B-E. Figure B-E: optical xy-sections $(206$ *206 $\mu \mathrm{m})$ of a common carotid artery, recorded at four $z$ positions in the vessel wall $(z$ $=0 \mu \mathrm{m}$ on the outside of the vessel). Fig. $B, z=12 \mu \mathrm{m}$; fig. $C, z=27 \mu \mathrm{m}$; fig. $D, z=45$ $\mu \mathrm{m}$; fig. $E, z=57 \mu \mathrm{m}$. White bars represent $20 \mu \mathrm{m}$. B shows the tunica adventitia with collagen lsecond harmonic generation of collagen, blue), and several thin elastin fibers (red). Furthermore, fibroblast-like cell nuclei (white arrow)

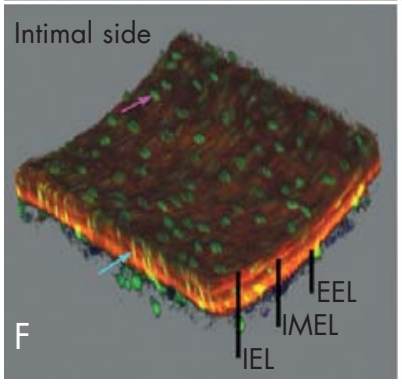
populate the tunica adventitia ( $B$ and $C)$. C and D represent the tunica media, where elastin forms the external elastic lamina (EEL, red), which is compact of structure and completely surrounds the "cigar-shaped" vSMC nuclei (blue arrow). In D the external elastic lamina (yellow dotted lines) and intermediate elastic lamina (IMEL, more intense red bands, pink dotted lines) are visible. E represents the tunica intima in the centre and shows the external elastic lamina (marked by yellow dotted lines), the intermediate elastic lamina (marked by pink dotted lines) which separates two layers of vSMCs, the internal elastic lamina (IEL, surrounded by blue dotted line), vSMCs (cigar shaped nuclei), and a monolayer of (elliptical) endothelial cell nuclei (pink arrow). F is a 3D-reconstruction (size: 206 * 206 * 59 pm) of the same stack of images representing the intimal side of the vessel. All three elastic laminae (IEL, IMEL, EEL) are visible at the transversal edges of the object. Endothelial cell nuclei (pink arrow) are positioned on top of the (uniformly shaped) internal elastic lamina (IEL, red), vSMCs (blue arrow) are visible at the edges of the reconstruction. A small part of the tunica adventitia visible at the bottom of the reconstruction shows collagen (blue) and fibroblasts. 
$x$

A

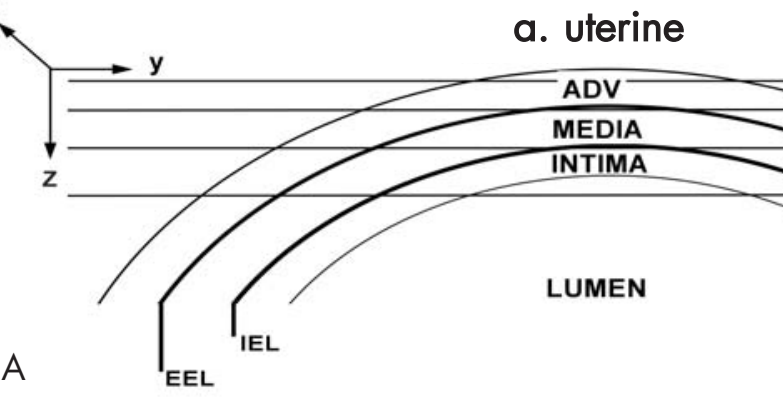

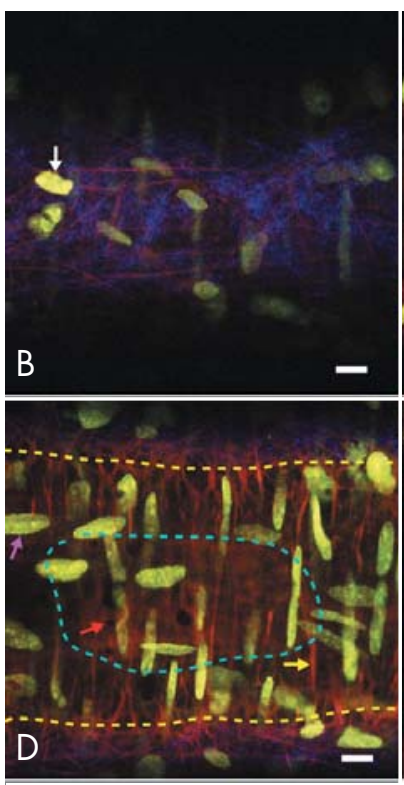

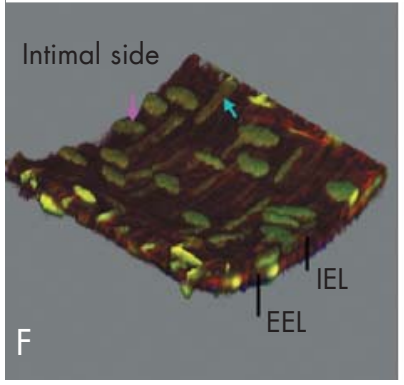

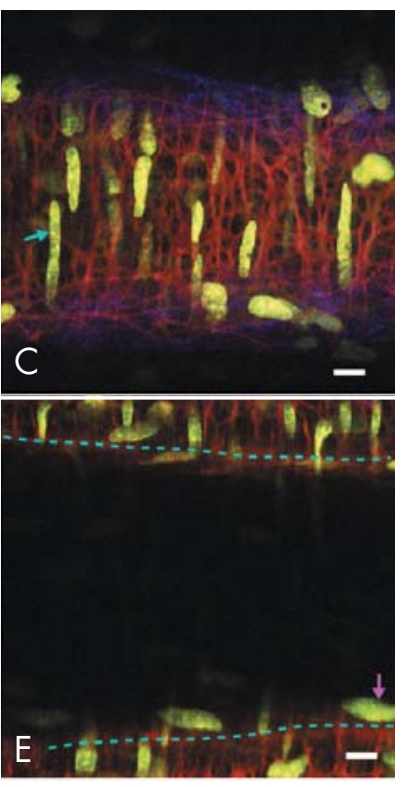

Figure 6:

A: schematic view of the arterial wall representing the positions of the $x y$-sections shown as 6 B-E. Figures 6 B-E: optical xy-sections 1137 * $137 \mu \mathrm{m})$ of a mounted muscular (uterine) artery, recorded at four $z$-positions in the artery $(z=0 \mu m$ on the outside of the vessel). $B, z=8$ $\mu \mathrm{m} ; \mathrm{C}, \mathrm{z}=15 \mu \mathrm{m} ; \mathrm{D}, \mathrm{z}=20 \mu \mathrm{m}$; $E, \quad z=30 \mu \mathrm{m}$. White bar represents $10 \mu \mathrm{m}$. B represents the tunica adventitia with collagen (SHG, blue), nuclei of fibroblast-like cells (white arrow), and thin elastin fibers (red). C represents the start of the tunica media. The external elastic lamina (EEL) is clearly visible in red and has a meshlike structure with a single layer of "cigar-shaped" vSMC nuclei (blue arrow) positioned directly underneath. The outside of the artery shows collagen (blue) and fibroblast-like cells. D is a section made deeper in the tunica media, which also contains part of the internal elastic lamina (IEL, surrounded by blue dotted line). In the tunica media, vSMC nuclei are surrounded by elastin fibers (yellow arrow) which are connected to the external (marked by yellow dotted lines) and internal elastic lamina, visible in the center. The IEL is uniformly shaped and contains several fenestrations (red arrow) with underneath, a monolayer of (elliptical) endothelial cell nuclei (pink arrow). E contains part of the tunica intima and the lumen. Endothelial cell nuclei (pink arrow) are visible on the luminal side and vSMC nuclei on the subluminal side of the internal elastic lamina (marked by blue dotted lines). F is a 3D-reconstruction (displayed artery size $137 * 137 * 23 \mu \mathrm{m}$ ) of the $z$-stack exposing the intimal side of the muscular artery. Both elastic layers (EEL and IEL), vSMC nuclei (blue arrow), and endothelial cell nuclei (pink arrow) can be distinguished at the transversal edges of the reconstruction. Endothelial cell nuclei are clearly visible on top of the internal elastic layer (red). Furthermore, vSMC nuclei weakly shine through the IEL (due to large difference in intensity between the IEL and VSMCs) and are visible when viewed from the luminal site. 

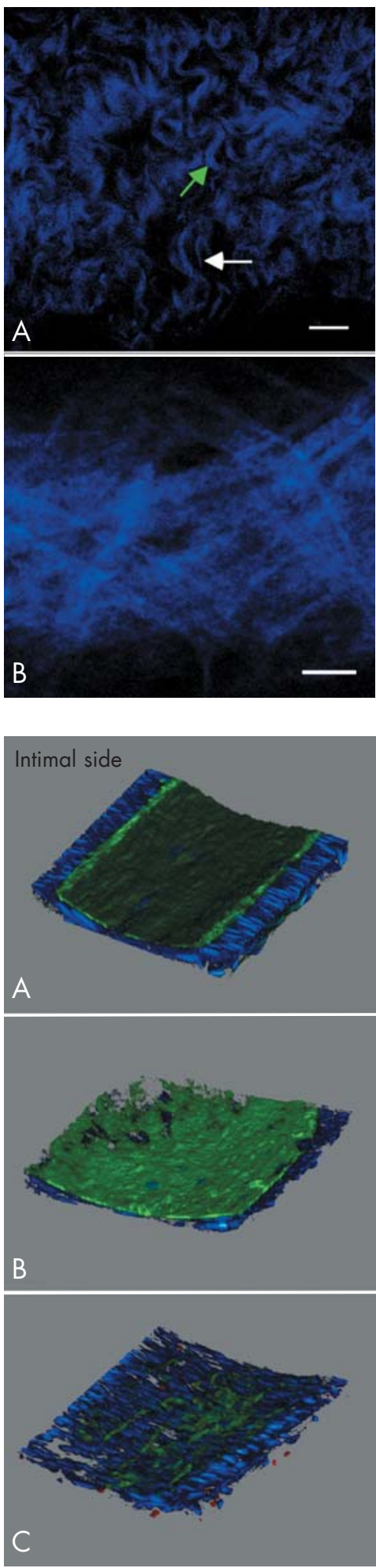

\section{Figure 7}

A-B: Second harmonic generation (SHG, blue) of collagen in xy-sections at comparable z-position in the tunica adventitia of an elastic (carotid) artery (A) and a muscular (uterine) artery (B), both pressurized and lengthadjusted. In the carotid artery (A), thin undulating collagen fibers (white arrow) rearrange into form thicker clustered collagen fibers (green arrow). In the muscular artery (B), fibers are less undulated and more difficult to distinguish (SHG-signal is weak when compared with the SHG-signal originating from the carotid artery). Bars represent $20 \mu \mathrm{m}$.

\section{Figure 8}

Examples of the glycocalyx in a mesenteric artery (A) and a carotid artery (B), labeled with WGA-FITC (green), SYTO41 (nuclei, blue) and PI (nuclei of cells with a compromised cell membrane, red). B-C; glycocalyx of a carotid artery labeled with WGA-FITC (green), SYTO4 1 (nuclei of vital cells, blue) and PI before (B) and after (C) treatment with hyaluronidase. The coverage of the endothelial cell layer by glycocalyx is drastically reduced after treatment with hyaluronidase. 


\section{References}

1. Hilgers, R.H., S. Bergaya, P.M. Schiffers, et al., Uterine Artery Structural and Functional Changes During Pregnancy in Tissue Kallikrein-Deficient Mice. Arterioscler Thromb Vasc Biol, 2003;23(10): 1826-1832.

2. Arribas, S.M., C. Hillier, C. Gonzalez, et al., Cellular aspects of vascular remodeling in hypertension revealed by confocal microscopy. Hypertension, 1997;30(6):1455-64.

3. Hilgers, R.H., J. Todd, Jr., and R.C. Webb, Regional heterogeneity in acetylcholine-induced relaxation in rat vascular bed: role of calcium-activated K+ channels. Am J Physiol Heart Circ Physiol, 2006;291(1):H216-22.

4. van Zandvoort, M., W. Engels, K. Douma, et al., Two-photon microscopy for imaging of the (atherosclerotic) vascular wall: a proof of concept study. J Vasc Res, 2004;41 (1):54-63.

5. Denk, W., J.H. Strickler, and W.W. Webb, Two-photon laser scanning fluorescence microscopy. Science, 1990;248(4951):73-6.

6. Masters, B.R. and P.T. So, Antecedents of two-photon excitation laser scanning microscopy. Microsc Res Tech, 2004;63(1):3-11.

7. de Grauw, C., M. van Zandvoort, M. oude Egbrink, et al., Two-photon lifetime imaging of blood and blood vessels. Proc. SPIE, 2001;4262:171-176.

8. Konig, K., Multiphoton microscopy in life sciences. J Microsc, 2000;200 ( Pł 2):83-104.

9. Ferrara, D.E., D. Weiss, P.H. Carnell, et al., Quantitative 3D fluorescence technique for the analysis of en face preparations of arterial walls using quantum dot nanocrystals and twophoton excitation laser scanning microscopy. Am J Physiol Regul Integr Comp Physiol, 2006;290(1):R 1 14-23.

10. Boulesteix, T., A.M. Pena, N. Pages, et al., Micrometer scale Ex Vivo multiphoton imaging of unstained arterial wall structure. Cytometry A, 2006;69(1):20-6.

11. Parasassi, T., W. Yu, D. Durbin, et al., Two-photon microscopy of aorta fibers shows proteolysis induced by LDL hydroperoxides. Free Radic Biol Med, 2000;28(1 1):1589-97.

12. Martinez-Lemus, L.A., M.A. Hill, S.S. Bolz, et al., Acute mechanoadaptation of vascular smooth muscle cells in response to continuous arteriolar vasoconstriction: implications for functional remodeling. Faseb J, 2004;18(6):708-10.

13. Manconi, F., E. Kable, G. Cox, et al., Whole-mount sections displaying microvascular and glandular structures in human uterus using multiphoton excitation microscopy. Micron, 2003;34(8):351-8.

14. Dorph-Petersen, K.A., J.R. Nyengaard, and H.J. Gundersen, Tissue shrinkage and unbiased stereological estimation of particle number and size. J Microsc, 2001;204(P+ 3):232-46.

15. Briones, A.M., J.M. Gonzalez, B. Somoza, et al., Role of elastin in spontaneously hypertensive rat small mesenteric artery remodelling. J Physiol, 2003;552(Pt 1):185-95.

16. Seyama, Y. and H. Wachi, Atherosclerosis and matrix dystrophy. J Atheroscler Thromb, 2004; 11 (5):236-45.

17. Karnik, S.K., B.S. Brooke, A. Bayes-Genis, et al., A critical role for elastin signaling in vascular morphogenesis and disease. Development, 2003;130(2):411-23. 
18. Dao, H.H., R. Essalihi, C. Bouvet, et al., Evolution and modulation of age-related medial elastocalcinosis: impact on large artery stiffness and isolated systolic hypertension. Cardiovasc Res, 2005;66(2):307-17.

19. Weinbaum, S., X. Zhang, Y. Han, et al., Mechanotransduction and flow across the endothelial glycocalyx. Proc Natl Acad Sci U S A, 2003;100(13):7988-95.

20. Constantinescu, A.A., H. Vink, and J.A. Spaan, Endothelial cell glycocalyx modulates immobilization of leukocytes at the endothelial surface. Arterioscler Thromb Vasc Biol, 2003;23(9): 1541-7.

21. Vink, H., A.A. Constantinescu, and J.A. Spaan, Oxidized lipoproteins degrade the endothelial surface layer: implications for platelet-endothelial cell adhesion. Circulation, 2000;101(13):1500-2.

22. van den Berg, B.M., J.A. Spaan, T.M. Rolf, et al., Atherogenic region and diet diminish glycocalyx dimension and increase intima-to-media ratios at murine carotid artery bifurcation. Am J Physiol Heart Circ Physiol, 2006;290(2):H915-20.

23. Dickhout, J.G. and R.M. Lee, Structural and functional analysis of small arteries from young spontaneously hypertensive rats. Hypertension, 1997;29(3):781-9.

24. Frobert, O., E.O. Mikkelsen, and J.P. Bagger, The influence of transmural pressure and longitudinal stretch on K+- and Ca2+-induced coronary artery constriction. Acta Physiol Scand, 1999;165(4):379-85.

25. Zoumi, A., X. Lu, G.S. Kassab, et al., Imaging coronary artery microstructure using secondharmonic and two-photon fluorescence microscopy. Biophys J, 2004;87(4):2778-86.

26. Campagnola, P.J., A.C. Millard, M. Terasaki, et al., Three-dimensional high-resolution secondharmonic generation imaging of endogenous structural proteins in biological tissues. Biophys J, 2002;82(1 Pt 1):493-508.

27. Zoumi, A., A. Yeh, and B.J. Tromberg, Imaging cells and extracellular matrix in vivo by using second-harmonic generation and two-photon excited fluorescence. Proc Natl Acad Sci U S A, 2002;99(17): 11014-9.

28. Lawrenson, J.G., A.R. Reid, and G. Allt, Corneal glycoconjugates: an ultrastructural lectingold study. Histochem J, 1998;30(1):51-60.

29. Henry, C.B. and B.R. Duling, Permeation of the luminal capillary glycocalyx is determined by hyaluronan. Am J Physiol, 1999;277(2 Pt 2):H508-14.

30. Heeneman, S., J.P. Cleutjens, B.C. Faber, et al., The dynamic extracellular matrix: intervention strategies during heart failure and atherosclerosis. J Pathol, 2003;200(4):516-25.

31. Gauderon, R., P.B. Lukins, and C.J. Sheppard, Optimization of second-harmonic generation microscopy. Micron, 2001;32(7):691-700.

32. Dora, K.A., S.L. Sandow, N.T. Gallagher, et al., Myoendothelial Gap Junctions May Provide the Pathway for EDHF in Mouse Mesenteric Artery. J Vasc Res, 2003;40(5):480-490.

33. Sandow, S.L., K. Goto, N.M. Rummery, et al., Developmental changes in myoendothelial gap junction mediated vasodilator activity in the rat saphenous artery. J Physiol, 2004;556(Pt 3):875-86. 
34. Hill, C.E., J.K. Phillips, and S.L. Sandow, Heterogeneous control of blood flow amongst different vascular beds. Med Res Rev, 2001;21(1):1-60.

35. Krahn, K.N., C.V. Bouten, S. van Tuijl, et al., Fluorescently labeled collagen binding proteins allow specific visualization of collagen in tissues and live cell culture. Anal Biochem, 2006;350(2):177-85.

36. Luft, J.H., Fine structures of capillary and endocapillary layer as revealed by ruthenium red. Fed Proc, 1966;25(6):1773-83.

37. van den Berg, B.M., H. Vink, and J.A. Spaan, The endothelial glycocalyx protects against myocardial edema. Circ Res, 2003;92(6):592-4.

38. Mulivor, A.W. and H.H. Lipowsky, Inflammation- and ischemia-induced shedding of venular glycocalyx. Am J Physiol Heart Circ Physiol, 2004;286(5):H1672-80.

39. van Haaren, P.M., E. VanBavel, H. Vink, et al., Localization of the permeability barrier to solutes in isolated arteries by confocal microscopy. Am J Physiol Heart Circ Physiol, $2003 ; 285(6): \mathrm{H} 2848-56$.

40. Pries, A.R., T.W. Secomb, and P. Gaehtgens, The endothelial surface layer. Pflugers Arch, 2000;440(5):653-66.

41. Ghitescu, L. and M. Robert, Diversity in unity: the biochemical composition of the endothelial cell surface varies between the vascular beds. Microsc Res Tech, 2002;57(5):381-9. 
Chapter 3 


\section{Chapter 4}

\section{Imaging Collagen in Intact Viable Healthy and Atherosclerotic Arteries using Fluorescently-Labeled CNA35 and Two-Photon Laser Scanning Microscopy.}

Molecular Imaging, 2007;6(4):247-60.

R.T.A. Megens ${ }^{a}$; M.G.A. oude Egbrink ${ }^{\text {b}}$; J.P.M.Cleutjens ${ }^{c}$; M.J.E. Kuijpers ${ }^{\text {d; }}$ P.H.M. Schiffers ${ }^{e}$; M. Merkx ${ }^{\text {; }}$ D.W. Slaaf ${ }^{a}$, f; M.A.M.J. van Zandvoort ${ }^{\circ}$.

Departments of 'Biophysics, 'Physiology, 'Pathology, 'Biochemistry, and ePharmacology, Cardiovascular Research Institute Maastricht, Maastricht University, P.O. Box 616, 6200MD Maastricht, the Netherlands. 'Department of Biomedical Engineering, Technical University Eindhoven, P.O. Box 513, 5600MB, Eindhoven, the Netherlands. 


\section{Abstract}

We evaluated CNA35 as collagen marker in healthy and atherosclerotic arteries of mice both after ex vivo and in vivo administration and as molecular imaging agent for detection of atherosclerosis.

CNA35 conjugated with fluorescent Oregon Green 488 (CNA35/OG488) was administered ex vivo to mounted viable muscular (uterine), elastic (carotid) and atherosclerotic (carotid) arteries and fresh arterial rings. Two-photon microscopy was used for imaging. CNA35/OG488 labeling in healthy elastic arteries was compared with collagen type I, III, and IV antibody labeling in histological sections. For in vivo labeling experiments CNA35/OG488 was injected intravenously in $\mathrm{C} 57 \mathrm{BL} 6 / \mathrm{J}$ and $\mathrm{ApoE}^{-1 /}$ mice.

Ex vivo CNA35/OG488 strongly labeled collagen in tunica adventitia, media, and intima of muscular arteries. In healthy elastic arteries, tunica adventitia was strongly labeled but labeling in tunica media and intima was prevented by endothelium and elastic laminae. Histology confirmed affinity of CNA35 for type I, III, and IV collagen in arteries. Strong CNA35/OG488 labeling was found in atherosclerotic plaques. In vivo applied CNA35/OG488 minimally labeled tunica intima of healthy carotid arteries. Atherosclerotic plaques in ApoE $\%$ mice exhibited large uptake. CNA35/OG488 imaging in organs revealed endothelium as limiting barrier for in vivo uptake. Conclusion: CNA35/OG488 is a good molecular imaging agent for atherosclerosis. 


\section{Introduction}

Collagen is a major constituent of the extracellular matrix in vasculature and is important for maintaining vascular integrity and stability [1-3]. In advanced atherosclerosis, collagen content is considered a major determinant for plaque stability [2-5]. Furthermore, collagen is one of the structural determinants for arterial stiffness $[1,6,7]$, which is associated with ageing $[6,7]$, hypertension [8], and diabetes [9]. Insight in vascular collagen structure, distribution, function, and content may lead to a better understanding of the pathogenesis of these vascular diseases. Therefore, a method to visualize collagen architecture in viable intact arteries is essential.

Imaging of vascular collagen in viable arteries excludes (immuno) histochemistry because this requires fixation or freeze-thawing to obtain arterial sections. Moreover, routinely used histological collagen stains such as Picrosirius Red or antibodies against specific types of collagen have limited applicability for viable samples due to staining procedures required or limited depth penetration into thick tissues. Required are thus both fluorescent marker that specifically labels collagen in viable arteries and a microscopy method that combines large penetration depth with optical sectioning. This combination will allow detailed imaging of structures deep in arterial tissue and 3-dimensional reconstructions.

Two-photon laser scanning microscopy (TPLSM) is established as an imaging modality which allows structural and functional imaging of viable intact arteries at cellular level with good penetration depth and optical sectioning [10-14]. Arterial wall collagen can directly be visualized using its Second Harmonic Generation (SHG) resulting from the TPLSM pulsed laser excitation [10, 12, 1517]. However, in contrast to small arteries [17], detection of SHG signal originating from collagen in the tunica media and intima in viable large arteries of mice is rather difficult [10]. Recently, Krahn and colleagues developed a collagen-specific fluorescent probe based on CNA35 [18]. CNA is a collagen binding adhesion protein found in Staphylococcus aureus and involved in wound infection caused by these pathogenic bacteria $[19,20]$. The collagen binding part of this transmembrane protein is called CNA35 and consists of the first two soluble domains, N1 and N2. Multiple binding sites have previously been observed for binding of CNA35 to both type I and type II collagens [19, 21]. Apparent $K_{d}$ 's (dissociation constant) ranging from 20 $\mathrm{nM}$ to $30 \mu \mathrm{M}$ have been reported, depending on the specific type of collagen [18-22]. Based on the recently determined X-ray structure of CNA35 in the presence of a short collagen triple helix, the so-called "collagen hug model" has been proposed [22]. According to this model binding of collagen is 
initiated by a low-affinity interaction between the collagen triple helix and a complementary trench located on the N2 domain. The N1 domain is not yet involved at this stage. The next step is reorientation and stabilization of the N1 domain as a result of which CNA35 is wrapping around the bound collagen. Finally, a C-terminal extension of the N2 domain stretches towards the N1 domain forming a $\beta$ strand that complements one of the $\beta$ sheets of the $N 1$ domain.

As a collagen marker, CNA35 has several potential advantages over existing collagen markers [18]: (1) CNA35 can be applied to both viable and freezethawed tissues; (2) Anti-bodies are more expensive. This excludes in vivo experiments which require large amounts of antibody; 3 ) collagen antibodies mostly are specific for one type of collagen. While this may be advantageous for histology, it is a serious drawback for the detection of overall collagen content; (4) CNA35 is known to bind reversibly to its target, which is important for a vital staining to avoid functional effects; (5) CNA35 conjugated with Oregon Green 488 (CNA35/OG488) yields a much stronger signal than SHG, allowing visualization of much smaller, newly formed fibrils; (6) CNA35 is approximately five times smaller than antibodies against collagen, which could be beneficial for penetration into thick viable samples; (7) CNA35 can easily be conjugated with contrast agents making it suitable for a range of imaging techniques.

The feasibility of collagen labeling with CNA35 conjugated with fluorescent Oregon Green 488 (CNA35/OG488) has already been shown in cell cultures and native tissues by Krahn et al. [18]. Furthermore, they verified that CNA35 has a high affinity for type I, III, and IV collagen [18], but not for other types of collagen or other extracellular matrix proteins, such as elastin, fibrin and laminin. Collagen types I, III, and IV are the most abundant collagens in the vessel wall [23] which are also associated with atherosclerosis and plaque progression [2, 5]. Type I and III collagen are fibrilar collagens [2] mainly located in the tunica adventitia and media [23]. Type IV collagen, a basement membrane associated type of collagen [2], is mainly located in the tunica media, surrounding the vascular smooth muscle cells (vSMCs) and in the tunica intima where it forms the general scaffold of the basement membrane [2].

Goal of this study is to determine the ex vivo and in vivo applicability of CNA35/OG488 for imaging of collagen in viable mounted arteries using TPLSM. To this purpose, we have applied CNA35/OG488 ex vivo to fresh transversal arterial rings and mounted healthy muscular (uterine), elastic (carotid) arteries, and atherosclerotic (carotid) arteries. Finally, CNA35/OG488 was administered in vivo in order to investigate CNA35/OG488 uptake in healthy and atherosclerotic arteries (and organs) 
and to determine its feasibility as a molecular imaging agent for detection of atherosclerosis.

\section{Methods}

\section{Animals}

All experiments were permitted by the local ethics committee on use of laboratory animals. Procedures were in accordance with institutional guidelines. For ex vivo experiments, euthanasia was performed by a mixture of $\mathrm{CO}_{2}$ and $\mathrm{O}_{2}$, after which arteries were isolated. Carotid artery segments (common part and bifurcation) were excised from 20-22 weeks old C57BL6/J $(n=6)$ and $\mathrm{ApoE}^{\%}(\mathrm{n}=6)$ mice (Charles River, Maastricht, the Netherlands). C57BL6/J mice were fed a chow diet; ApoE $\%$ mice were fed a western diet for 14 weeks (Western Diet 4021.06, Hope Farms, Woerden, the Netherlands). Uterine (mid-section, $n=3$ ) arteries were obtained from 12-16 weeks old nonpregnant female C57BL6/J mice (Charles River) on a chow diet.

For in vivo labeling experiments, two C57BL6/J (fed a chow diet) and five ApoE$\%^{\%}$ (fed a western diet) mice (20-22 weeks) were anesthetized by subcutaneous administration of a mixture of xylazine (Xylazin ${ }^{\circledast}$, Ceva Sante Animale BV, Naaldwijk, the Netherlands; $15 \mathrm{mg} / \mathrm{kg}$ body weigth b.w.) and ketamine (Nimatek ${ }^{\circledR}$, Eurovet, Cuijk, the Netherlands; $75 \mathrm{mg} / \mathrm{kg} \mathrm{b.w.).} \mathrm{After}$ CNA35/OG488 infusion, animals were euthanized (overdose of Ketamin/ Xylazin), blood was withdrawn, and carotid arteries, kidneys, liver, spleen, lungs, and the heart were isolated.

\section{Preparation and staining procedures}

\section{CNA35/OG488 administration ex vivo}

\section{Fresh transversal arterial rings}

To test whether CNA35/OG488 has affinity for collagens in all tunicae of arteries, $1 \mathrm{~mm}$ thick rings were cut off from isolated common carotid arteries $(n=3)$. These rings were incubated for 60 minutes with $1.5 \mu M$ CNA35/OG488 in Hanks Balanced Salt Solution (HBSS, pH 7.4) containing in $\mathrm{mM}: \mathrm{NaCl} 144, \mathrm{HEPES} 14.9$, glucose 5.5, $\mathrm{KCl} 4.7, \mathrm{CaCl}_{2} 2.5, \mathrm{KH}_{2} \mathrm{PO}_{4}$ 1.2 , and $\mathrm{MgSO}_{4}$ 1.2. Nuclei in one arterial ring were labeled with the DNA/RNA marker SYTO41 $\left(\lambda_{\text {max emission }}=480 \mathrm{~nm}\right.$, final concentration 1.5 $\mu \mathrm{M}$; Molecular Probes, Leiden, the Netherlands). After incubation the rings were embedded in $1.5 \%$ agarose gel dissolved in HBBS (Gibco BRL 15510027; Invitrogen, Breda, the Netherlands) for stabilization during imaging [12]. In these rings, elastic bands were visualized using autofluorescence properties of elastin [17]. 


\section{Whole mount arteries}

To explore the characteristics of CNA35/OG488 in structurally intact and viable arteries, muscular (uterine) and elastic (carotid) arteries were mounted in a perfusion chamber, as described previously [10, 24]. Briefly, isolated arteries were mounted on two glass micropipettes in a perfusion chamber (volume $10 \mathrm{ml}$; IDEE BV, Maastricht, the Netherlands). The whole system was filled with HBSS. Correction for vascular shortening due to isolation was performed at a transmural pressure of $100 \mathrm{mmHg}$ by stretching the vessel until its bends were removed. Subsequently, vessels were imaged at a transmural pressure of $80 \mathrm{mmHg}$ in order to mimic physiological conditions. Viability was maintained for up to 4 hours [10]. All ex vivo experiments were performed at room temperature. In case of carotid artery bifurcations the mounting procedure was to a large extent similar to the one used for common carotid arteries, except that the internal branch was mounted on a glass micropipette in the perfusion chamber, while the external branch and other side branches of the bifurcation were ligated after which intraluminal pressure was applied (40$80 \mathrm{~mm} \mathrm{Hg}$, depending on the maximum pressure the ligations of the side branches could withstand) and length was corrected. TPLSM imaging was restricted to the central portion of the mounted vessel segment, which included the bifurcation.

For specific labeling of vascular structures and cells and to verify arterial viability, several fluorescent markers were applied: CNA35 conjugated with Oregon Green 488 (OG 488, $\lambda_{\text {max emission }}=510 \mathrm{~nm}$ ) was used as a collagen marker (final concentration 1.5 $\mu$; kindly provided by Technische Universiteit Eindhoven, Eindhoven, the Netherlands) [18]. CNA35/OG488 contains approximately three $\mathrm{OG} 488$ labels per CNA35 protein [18]. Final CNA35/OG488 concentration was optimized by testing a range of concentrations from 1.0 to $5.0 \mu \mathrm{M}$ in elastic arteries. Nuclei of cells with an intact cell membrane were labeled with the viable cell membrane permeable DNA / RNA marker SYTO41 (final concentration $2.5 \mu \mathrm{M}$ ). Propidium lodide (PI, $\lambda_{\text {max emission }}=620 \mathrm{~nm}$, final concentration $1.5 \mu \mathrm{M}$, Molecular Probes) was used to assess cell membrane integrity and, hence, cell viability in mounted arteries. Elastin was labeled with eosin $\left(\lambda_{\max }\right.$ emission $=545 \mathrm{~nm}$, final concentration $0.5 \mu \mathrm{M}$, Molecular Probes) [12]. All labels were applied both intra- and extraluminally. Fluorescent probes were diluted in HBSS and added 30 minutes prior to start of image acquisition. To avoid additional manipulations of the artery, fluorescent probes remained present during image acquisition. Presence of the probes did not alter their labeling characteristics, the viability of the arteries, or reduce the image quality. 


\section{Vascular damage}

To further investigate CNA35/OG488 penetration in the arterial wall, mounted elastic arteries were mechanically damaged $(n=2)$ by pinching the artery (two pinches, 3 seconds per pinch) at one position using a fine forceps (FST \#5, biology tip, FST, Vancouver, Canada). CNA35/OG488 was applied intraluminally in both arteries; labels remained present during and after pinching. Nuclei of cells with an intact cell membrane were labeled with the DNA / RNA marker SYTO41 (final concentration $2.5 \mu \mathrm{M}$ ); nuclei of cells with lost membrane integrity were labeled with PI. One artery was additionally labeled with eosin for visualization of elastin. Images were recorded both immediately and 30 minutes after pinching.

\section{Histology}

At the end of the ex vivo experiments, arteries were fixed luminally and abluminally with formalin (10\% in HBSS) at a transmural pressure of $80 \mathrm{mmHg}$ for 24 hours. Following fixation, arteries were embedded in paraffin [25] after which $5 \mu \mathrm{m}$ thick longitudinal and transversal sections were cut. Before the start of histological staining procedures, sections were deparaffinized in xylene and rehydrated in ethanol. Endogenous peroxidase activity was blocked by incubation with methanol plus $3 \%$ hydrogen peroxide for 15 minutes. Sections were washed with Tris-Buffered Saline (TBS) containing in mmol/l: $\mathrm{NaCl}$ 136.9, Trizma base 5.0, 1 \% BSA, and 0.1 \% Tween 20, pH 7.6) and treated with $0.1 \%$ pepsin in $0.01 \mathrm{M} \mathrm{HCL}$ for 30 minutes. After washing, sections were incubated with CNA35/OG488 (1 $\mu M)$, primary rabbit anti-human polyclonal antibodies against collagen type I or III (both 1:10, Biogenesis, Kingston, $\mathrm{NH}$, USA), or anti-human monoclonal antibody against collagen type IV (1:400, [25]) for 60 minutes at room temperature. Antibodies and CNA35/OG488 were dissolved in TBS. The human antibodies were shown to stain mouse collagens as well with similar specificity (unpublished data). After washing with TBS, CNA35/OG488 treated sections were coverslipped using Entellan (Merck, Darmstadt, Germany). Sections treated with a primary antibody were washed after which secondary biotin-labeled swine anti-rabbit antibody was applied (1:1000; DAKO, Glostrup, Denmark). Subsequently after washing, sections were incubated with Streptavidin-ABC-HRP (1:500; DAKO) for 30 minutes at room temperature. DAB (DAKO) treatment (10 minutes at room temperature) was used for chromogenic development. Sections were coverslipped using Entellan.

CNA35/OG488 was diluted in a standard physiological salt infusion fluid 
(Braun AG, Melsungen, Germany) to a final concentration of $19 \mu \mathrm{M}$ or $38 \mu \mathrm{M}$. Dependent on the desired concentration, one or two boluses of $200 \mu$ each were infused via the tail vein of the anaesthetized mice. Several concentrations were tested: Several administration doses were tested: $200 \mu$ at $19 \mu M(n=2$;

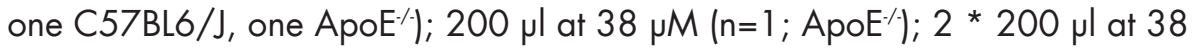
$\mu \mathrm{M}\left(\mathrm{n}=4\right.$; one $\mathrm{C} 57 \mathrm{BL6} / \mathrm{J}$, three $\left.\mathrm{ApoE}^{-/}\right)$. In the latter, CNA35/OG488 was infused in two boluses of $200 \mu \mathrm{l}$ each (instead of one bolus of $400 \mu \mathrm{l}$ ) in order to enhance the total circulation time of the probe. Moreover, a volume of 400 $\mu l$ is too high a volume load for a single infusion in mice. Since $38 \mu M$ was the maximal concentration of the available stock solution, further increase of the concentration required an increase of the total infusion volume above $400 \mu \mathrm{l}$ and was therefore not used. Incubation time per infusion was 45 minutes; total incubation time was 90 minutes. To exclude labeling effects of CNA35/OG488 after euthanasia, contact of the two carotid arteries with spoiled blood was avoided. Both carotid arteries were mounted as described above. After mounting, anti-CD3 1 conjugated with Phycoerythrin (PE) was diluted in HBSS and applied intraluminally as a marker for endothelial cells $\left(\lambda_{\text {max emission }}=570 \mathrm{~nm}\right.$, final concentration $8 \mu \mathrm{g} / \mathrm{ml} ;$ BD Biosciences, Alphen aan den Rijn, the Netherlands). CNA35 content in the blood was studied in blood smears. Kidneys, liver, spleen, lungs, and heart were washed in HBSS and embedded in agarose gel (1.5\% dissolved in HBBS).

\section{Imaging}

The microscope setup used for imaging of viable arteries (and organs) has been described previously [10, 12]. In short, a Biorad 2100MP TPLSM was used (Biorad, Hemel Hempstead, UK), coupled to a Nikon E600FN microscope (Nikon Corporation, Tokyo, Japan). The excitation source was a pulsed (140 femtoseconds) Ti:sapphire laser (Spectra Physics Tsunami, Mountain View, USA), tuned and mode-locked at $800 \mathrm{~nm}$. Laser light reached the sample through a $60 \mathrm{X}$, NA 1.0 water dipping objective (working distance $2 \mathrm{~mm}$, maximum field of view $206 \mu \mathrm{m} * 206 \mu \mathrm{m})$. When required, resolution was enhanced by optical zoom (consequently, the field of view is reduced). To avoid thermal and photochemical damage to the sample, intensity of the excitation laser was kept as low as possible [26].

For simultaneous imaging of up to three different fluorescent markers, filters were used to split the emitted fluorescence light into three different wavelength ranges. Each wavelength range corresponded to (part) of the emission spectrum of the fluorophores used and was collected by one of three Photo Multiplier Tubes (PMT): SHG 400-420 nm (PMT I); SYTO4 1, 460-480 nm (PMT I); CNA35/OG488, 520-550 nm (PMT II); eosin, 530-560 nm (PMT III); PI, 
570-610 nm (PMT III); CD31/PE, 570-610 nm (PMT III). For simultaneous imaging of a combination of fluorescent markers, each PMT was tuned for minimal bleed-through of fluorescent signal of the used markers.

From each PMT, separate images of 512 * 512 pixels were obtained, saved, and combined into an RGB-image. Frame rate was $0.1 \mathrm{~Hz}$ with a pixel dwell time of $39 \mu \mathrm{s}$, or $0.3 \mathrm{~Hz}$ with a pixel dwell time of $0.12 \mu \mathrm{s}$ combined with Kalman filtering for noise reduction ( $n=3$ cycles). Optical sections were collected in the xy-direction; series of $x y$-sections at successive depth positions (z-stack) were collected for reconstruction of 3D-images [10]. Incorrect alignment of the micropipettes in the perfusion chamber occasionally caused imaging of the arteries in a slightly oblique plane. Atherosclerotic plaque detection in the mounted vessel segments was performed by the use of bright field microscopy where plaques show up as dark and less translucent regions on a bright background (of healthy vessel segments). This initial detection was further supported by the 3D-capability of TPLSM which allows plaques to be identified as a deviation from the staining pattern as visible in the vessel wall of healthy carotid arteries.

Histological sections were imaged using a Leica DM 5000B microscope (Leica Microsystems $\mathrm{GmbH}$, Wetzlar, Germany) coupled to a Leica DC 300FX digital camera. A $100 \mathrm{X}$ oil objective (NA=1.4) was used.

Images of histological sections were analyzed using Qwin morphometry software (Leica, Cambridge, UK). Image analysis of TPLSM data was performed with the Image-Pro Plus 6.0 software (Media Cybernetics, Silver Spring, USA). 3D-reconstructions were performed using the 3D-constructor 5.1 package for Image-Pro Plus (Media Cybernetics).

\section{Results}

\section{Histology}

In order to verify the location of the three main types of collagen in large arteries of mice, histological sections of elastic arteries were prepared and labeled with collagen type I, III, or IV antibodies. As an example, a histological section specifically stained for type IV collagen is shown in figure la. Type IV collagen (brown stain) is clearly present in the tunica media between the EEL and intermediate elastic lamina (IMEL) and surrounding the vSMCs (green arrow). A thin sheet of type IV collagen (red arrow) is present at the luminal site of the tunica intima which corresponds with the endothelial basement membrane. No type IV collagen is visible in the tunica adventitia. The distribution of type I, III, and IV collagen as detected in these histological sections is illustrated schematically in figure 1b. Type I and III collagens were 
exclusively observed in the tunicae adventitia and media whereas type IV collagen was present in the tunicae media and intima but not in the tunica adventitia. Application of CNA35/OG488 on histological sections of elastic arteries revealed that CNA35 labeled collagen in all tunicae (fig. 1c) and demonstrated its potential as a collagen label in fixed arteries. The elastic laminae (blue) were not labeled by CNA35/OG488.

\section{Ex vivo CNA35/OG488 labeling in healthy arteries: where does CNA35/OG488 go to?}

\section{Mounted muscular and elastic arteries}

To establish the labeling characteristics of CNA35/OG488 in viable and structurally intact arteries, we show three xy-sections of a z-stack at comparable positions in the arterial wall of mounted, viable muscular (uterine, fig. 2a, c, e) and elastic (carotid, fig. 2b, $d, f$ ) arteries. 3D-reconstructions of both z-stacks are shown in figure $2 \mathrm{~g}$-h. Arteries were labeled for collagen Igreen, CNA35/OG488), elastin (red, eosin), and nuclei (blue, SYTO41). Note that in both arterial types the elastin structures do not exhibit any CNA35/OG488 labeling. The tunica adventitia is a layer which contains large amounts of type I and III collagen (fig. 1b) [23]. Figure 2a-b display collagen (green), several thin elastin fibers (red), and fibroblast-like cell nuclei (red arrow). In both muscular and elastic arteries (fig. 2a-b), collagen is clearly visible in the tunica adventitia, but in elastic arteries (fig. 2b) the adventitial collagen layer is thicker and the density of collagen fibers is higher, as already shown by SHG [10]. Also in the tunica media (fig. 2c, d), collagen labeling of elastic and muscular arteries differed significantly. In the muscular artery (fig. 2c), collagen fibers are clearly distinguishable between the "cigar-shaped" vSMC nuclei (green arrow), while in the elastic artery collagen labeling is completely absent in the tunica media (fig. 2d). Elastin (red) in the muscular arteries has a mesh-like structure in the EEL and in between the single layer of vSMCs (green arrow), long elastic fibers are visible. In the tunica media of the elastic artery (fig. 2d), collagen labeling is completely absent. The structure of elastin (red) is more compact and completely surrounds the two layers of vSMCs (green arrow). Finally, in the tunica intima of muscular arteries (fig. 2e) CNA35 labeling visualizes a (barely visible) hazy layer of collagen on the luminal side of the uniformly shaped internal elastic layer (IEL), with few small sites with increased intensity (pink arrow). These sites are located in and around fenestrae present in the IEL. Furthermore, endothelial cell nuclei (white arrow) and part of the lumen are noticable. In the tunica intima of an elastic artery (fig. 2f) endothelial cells are clearly observable. The IEL (red) is also uniformly shaped but contains less fenestrations. No hazy layer of collagen is observable. Furthermore, only 
few CNA35/OG488 labeled sites (encircled by white dotted lines) are visible between the vascular endothelial cells and IEL. Figure 3 shows that CNA35/OG488 labeled sites in the tunica intima of elastic arteries are mainly, but not exclusively, found in close proximity of (damaged) PI-positive endothelial cells (pink arrow). Also areas behind SYTO41 positive endothelial cells (green arrow) sometimes contain CNA35/OG488 (white arrow). PIstaining is indicative of loss of cell integrity and occasionally occurs as a consequence of the preparation and mounting procedure [10]. However, in the present setup the number of $\mathrm{Pl}$-positive cells in mounted and stained arteries remained low and stable for up to 4 hours after CNA35/OG488 incubation. Final CNA35/OG488 concentration, ranging from $1.0 \mu \mathrm{M}$ to $5.0 \mu \mathrm{M}$, did not influence labeling intensity and pattern in the tunica media and intima of either vessel type. By contrast, mechanically induced damage (pinching with a forceps) of elastic arteries resulted in a large increase of collagen labeling in the tunica intima. A bright collagen layer became visible at the damaged site, whereas in the undamaged area, such a collagen layer cannot be distinguished (fig. 4a). Furthermore, vSMCs (red arrow) and endothelial cells (white arrow) are only detectable in the undamaged area; the tunica media remained unlabeled by CNA35/OG488 in the damaged area (fig. 4a). Pinching with a forceps did not damage the elastin layers of the artery, as is illustrated in figure $4 \mathrm{~b}$. The IEL (homogeneous orange layer) in the damaged and undamaged area is comparable while the vSMCs (red arrow) are again only observable in the undamaged part of the artery (as in figure 4a). Adventitial collagen was unaltered at damaged sites (not shown).

\section{CNA35/OG488 labeling in fresh arterial rings}

Since in viable mounted elastic arteries CNA35/OG488 did not label collagen in the tunica media, we tested whether the internal and external elastic laminae limit the penetration of CNA35/OG488 into the tunica media of fresh arteries. To that end we labeled fresh rings of elastic arteries with CNA35/OG488. Indeed, CNA35/OG488 appeared to label collagen in the tunica media of these arterial rings. As shown in figure 5, CNA35/OG488 was observed adjacent to the elastic laminae (fig. $5 a$ ) and in between vSMCs (red arrow) in the tunica media (fig. 5b), which is in agreement with data from our histochemical experiments (fig. 1 b) and from literature [23]. As in mounted arteries, the tunica adventitia of fresh arterial rings also showed large uptake of CNA35/OG488 (fig. 5a). The tunica intima (fig. 5a) exhibited collagen labeling at the luminal site of the IEL, the location of the basement membrane which contains type IV collagen [2]. The three elastic laminae were not labeled with CNA35/OG488 but were visible in blue due to autofluorescence (fig. 5a). 


\section{Ex vivo labeling of atherosclerotic plaques}

In order to test the uptake of CNA35/OG488 in atherosclerotic plaques, we have applied CNA35/OG488 to mounted carotid arteries of ApoE $\%$ mice. Ex vivo administered CNA35/OG488 labeled all atherosclerotic plaques in the carotid arteries under investigation. Atherosclerotic plaques were typically located in the thickened tunica intima of the bifurcation and exhibited large uptake of CNA35/OG488. Figure 6a is an example of an optical section of an atherosclerotic plaque in the tunica intima. Collagen in the plaques had a matrix-like structure with an inhomogeneous intensity. Many disorderly arranged cells (red arrow) were present between the collagen network. Furthermore, large amounts of strongly labeled collagen were found in the shoulder of plaques which is marked by a red dotted line in figure 6b (3Dreconstruction of the shoulder area of an atherosclerotic plaque stained with CNA35/OG488). Interestingly, CNA35/OG488 labeling was also strongly increased in the tunica intima adjacent to the plaque, up to a distance of 150 $\mu \mathrm{m}$ from the edge of the atherosclerotic plaque (lower right part of the 3Dreconstruction in figure $6 \mathrm{~b}$ ). In healthy arteries, labeling in the tunica intima was almost absent (fig. 2f).

\section{In vivo labeling of healthy arteries and atherosclerotic plaques}

\section{Concentration of in vivo administered CNA35/OG488}

All tested doses obtained similar results in healthy and atherosclerotic arteries (and organs), but with differences in signal intensity. Compared with both applied lower doses (single bolus of $200 \mu \mathrm{l}$ at $19 \mu \mathrm{M}$ or $38 \mu \mathrm{M}$ each, data not

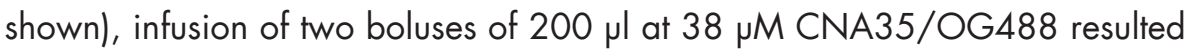
in the strongest signal intensity.

\section{Healthy elastic arteries}

In vivo administration of CNA35/OG488 did not result in collagen labeling in the tunica adventitia and tunica media in any section of the carotid artery of wildtype mice. In the tunica intima only very little CNA35/OG488 (green) uptake was detectable (fig. 7a). The blue signal is caused by autofluorescence from the elastic layers. This minimal uptake was located between the endothelial cells (red) and the IEL (inner thin blue layer) as is indicated by a white arrow in figure $7 b$; transversal view of a 3D-reconstruction of the same artery after subsequent labeling of the endothelial cells with anti-CD31/PE.

\section{Atherosclerotic plaques}

As in wildtype mice, intravenous administration of CNA35/OG488 did not result in collagen labeling in the tunica adventitia and media in elastic arteries 
of ApoE $\%$ mice (not shown). This is indicative of the inability of the luminally present probe to enter this medial layer in vivo in both healthy and atherosclerotic arteries. However, in $\mathrm{Apo} \mathrm{E}^{\%}$ mice in vivo administration of CNA35/OG488 resulted in widespread labeling of collagen in the atherosclerotic plaques and the tunica intima immediately adjacent to the plaques (fig. 7c), which is clearly different from the limited CNA35/OG488 labeling in healthy arteries (fig. 7a). The detected collagen in the plaque region again had a network-like shape and the plaque shoulder (red dotted line) exhibited intense CNA35/OG488 labeling (fig. 7c), as was the case in the ex vivo labeled atherosclerotic plaques. In vivo administered CNA35/OG488 stained all atherosclerotic plaques in the examined carotid arteries.

\section{Organs with different endothelial phenotypes}

In order to determine the role of the endothelium as a limiting barrier for CNA35/OG488 uptake, several organs of wildtype and ApoE $\mathrm{E}^{\prime}$ mice with different endothelial phenotypes were checked for presence of CNA35/OG488 after in vivo (intravenous) administration. Organs with fenestrated or discontinuous endothelium, such as spleen, liver, and kidneys, contained large amounts of CNA35/OG488, mostly localized in the vascular network. Figure $7 \mathrm{~d}$ displays a nephron which exhibits strong labeling in the glomeruli (I), Bowmans capsules (red arrow), and proximal tubuli (II). This probably results from filtration of CNA35/OG488 through the relative permeable endothelial layer. In contrast, organs with continuous endothelium like heart and lungs, no CNA35/OG488 uptake in the vessel wall was observed. Figure 7e displays (healthy) heart tissue in which CNA35/OG488 labeling was absent in the vasculature (yellow arrow) and in between myocytes (green arrow). CNA35/OG488 was only sporadically detected in full blood smears 45 minutes after in vivo administration.

\section{Discussion}

In this study we have visualized collagen in viable large arteries of control (C57BL6/J) and atherosclerotic (ApoE $\left.{ }^{\prime}\right)$ mice using the fluorescent collagen marker CNA35/OG488. TPLSM was applied ex vivo to visualize the distribution of this marker in mounted elastic (carotid) and muscular (uterine) arteries. The route of CNA35/OG488 administration, either ex vivo (intraluminal and extraluminal) or in vivo (intraluminal), strongly influences labeling patterns. After ex vivo administration, muscular arteries (uterine) of C57BL6/J mice exhibited labeling in all layers, while in elastic (carotid) arteries the tunica media remained unlabeled. Interestingly, in 22 weeks old ApoE $\%$ mice fed a western diet, all atherosclerotic lesions and immediately 
adjacent regions in the carotid artery contained large amounts of CNA35/OG488 labeled collagen. In vivo infusion of CNA35/OG488 in control mice resulted in almost complete absence of labeling in carotid artery, while in the $\mathrm{Apo}^{-1}$ mice exclusively the atherosclerotic lesions contained large amounts of CNA35/OG488 labeled collagen. These observations establish CNA35/OG488 as a potential molecular imaging agent for atherosclerotic lesions.

We have confirmed the presence of type I, III, and IV collagen in elastic arteries of mice. Both type I and III collagen (fibrilar collagens [2, 5, 27]) were found to be present in the tunicae adventitia and media, while type IV collagen (basement membrane component $[2,25]$ ) was present in the tunicae media and intima. Both histological sections and fresh transversal rings displayed uptake of CNA35/OG488 at collagen-containing sites in the arterial wall. The elastic laminae were not labeled with CNA35/OG488 and clearly detectable by their autofluorescence signal. This further demonstrates the low affinity of CNA35 for elastin, the other major component of the extra cellular matrix in arteries [23] confirming earlier findings of Krahn and colleagues in solid-phase binding assays [18]. These labeling characteristics together with the ease of application of CNA35 in both viable and non-viable arterial sections and the possibility to conjugate CNA35 with other (non)fluorescent contrast agents, might favor CNA35 as a routine label for collagen in arteries.

Ex vivo (intra- and extraluminal) administration of CNA35/OG488 to both mounted elastic and muscular arteries strongly labeled collagen in the tunica adventitia. Compared with a previous study using SHG [10], visualization of collagen in the tunica adventitia based on CNA35/OG488 labeling provides much better contrast.

Only weak spots of CNA35/OG488 were observed in the tunica intima of elastic arteries, which were mostly located behind PI-positive, non-viable cells. Therefore these spots in the tunica intima are probably an artifact of mounting procedure. In contrast, in muscular arteries a hazy CNA35/OG488 layer in the tunica intima was accompanied by few intense spots. The combination of the presence of subendothelial collagen labeling in figure la (histology, type IV collagen antibody), figure 1c (fresh arterial ring, CNA35/OG488 localized immediately on the luminal side of the IEL), figure $4 \mathrm{a}$ (mounted artery after pinching with a forceps shows enhanced uptake of CNA35/OG488 in exactly that layer) and figure 7b (after in vivo administration, CNA35/OG488 uptake is clearly visible behind the anti-CD3 1 stained endothelial cells) demonstrate that the diffuse layer in figures $2 e$ and $2 f$ is subendothelial collagen. It is known 
that endothelial cells are not the same throughout the cardiovascular system [28]. This may result in variations in permeability [28] which may account for the differences in intimal CNA35/OG488 uptake between elastic and muscular arteries. This hypothesis of endothelial cells as a barrier for probe uptake is supported by 2 findings: (1) the subendothelial collagen layer is strongly labeled after mechanical damage of a carotid artery, which results in a loss of endothelial cell integrity while leaving the IEL visually intact (fig. 4b) and (2) after in vivo administration of CNA35/OG488, organs with either discontinuous endothelial vascular coverage (liver, spleen) or fenestrated endothelium (kidney) exhibit labeling of collagen in subendothelial layers of blood vessels; in organs with continuous endothelium (heart, lungs [23, 29]) no such labeling was observed. One should realize that the endothelial barrier is important especially after intravenous administration in vivo. In contrast to ex vivo labeling, the probe is present at relevant concentrations only during a narrow time window. It circulates in the blood only for a limited time and with fast decaying concentration, as demonstrated by the fact that already 30 minutes after probe administration, CNA35/OG488 is no longer detectable in full blood.

The tunica media of muscular arteries contained CNA35/OG488 near the IEL and EEL, and in between vSMCs. In contrast, the tunica media in elastic arteries was not labeled by ex vivo administered CNA35/OG488, independent of the applied labeling concentration. It can be excluded that CNA35/OG488 is not sensitive for collagen in the tunica media. Indeed, the tunica media of fresh transversal rings and fixed histological sections of elastic arteries contained CNA35/OG488 labeled collagen. More likely, the absence of CNA35/OG488 uptake in the tunica media is caused by barriers the probe meets on diffusion through the artery. As indicated above, one of the probable barriers is the endothelial cell layer. However, while subendothelial intimal labeling by CNA35/OG488 was increased adjacent to areas with PI-positive endothelial cells (i.e., cells with a compromised membrane) the tunica media showed no increased labeling in those regions. Furthermore, vascular damage induced an increased uptake of CNA35/OG488 in the tunica intima, but not in the tunicae media or adventitia. These results indicate that although (viable) endothelial cells are determinants for ex vivo CNA35/OG488 labeling in the tunica intima, they are not the only barrier for uptake in the tunica media. More probably, the geometry and high density of elastin in elastic arteries as compared to muscular arteries [10] explains the differences in medial labeling between these vessels. Elastin is a known barrier for movement of water and solutes through the vessel wall [30]. Compared with muscular arteries, the geometry of the IEL and EEL in the thick elastic arteries is much more compact 
and the number and size of fenestrae is smaller (fig. 2) [10]. Indeed, in muscular arteries, CNA35/OG488 labeling in the tunica media seems to be in close proximity with fenestrae in the IEL. Furthermore, after mechanical damage of the carotid artery, the elastin layer seems to be unaffected with unaltered medial collagen staining (fig. 4b).

In contrast to healthy arteries, a large amount of CNA35/OG488 uptake was observed in all atherosclerotic lesions in ex vivo labeled mounted arteries of 20-22 weeks old ApoE $\%$ mice (fed a western diet). The borders (shoulders) of atherosclerotic plaques contained large amounts of collagen. Inside the plaque, a matrix-like network of collagen was detected. No CNA35/OG488 uptake was detected inside the lipid core. Although only little collagen is expected in the lipid core of advanced plaques [3 1], it cannot be excluded that lipids limit the penetration depth of CNA35/OG488. Furthermore, lipids also restrict the penetration depth of TPLSM. The IEL, which appeared to limit CNA35/OG488 uptake in the tunica media of ex vivo labeled control elastic arteries, cannot be the limiting factor of penetration depth in plaques, since plaques are mainly located in the tunica intima. Remarkably, an increase of CNA35/OG488 labeling in the tunica intima was detected in the arterial wall adjacent to a plaque. This suggests an increase in collagen content in these areas. It is indeed known that collagen content increases in parallel with progression of atherosclerosis [3, 5, 32]. However, increased permeability of endothelial cells in plaque regions [31, 33] may also cause stronger CNA35/OG488 labeling of the generally present collagen in the tunica intima in the area adjacent to atherosclerotic plaques.

In vivo administration (systemic infusion) of CNA35/OG488 in control mice resulted in barely any uptake in the healthy elastic (carotid) arteries. The tunica intima exhibited only very little uptake of CNA35/OG488, while the tunica media was unlabeled. Also collagen in the tunica adventitia was not labeled, which is a consequence of both the absence of extraluminal CNA35/OG488 labeling after in vivo administration and the inability of CNA35/OG488 to reach the tunica adventitia from the luminal side. In contrast, in vivo administration in atherosclerotic mice resulted in a massive CNA35/OG488 labeling of all atherosclerotic plaques. This result was very much alike to findings after ex vivo labeling, suggesting the absence of diffusion barriers. In both situations, the matrix-like network of the collagen in the plaques was visualized and increase of CNA35/OG488 uptake was detected in the arterial wall adjacent to the plaque. 
The minimal uptake of CNA35/OG488 in healthy elastic arteries and the high uptake in atherosclerotic arteries after in vivo administration allow differentiation between healthy and atherosclerotic sites and demonstrate the feasibility of CNA35 as a molecular imaging agent for atherosclerosis (although the exact pharmacokinetics of CNA35/OG488 are still unknown). Since collagen content and endothelial permeability are both determinants for plaque stability, the total CNA35 uptake may provide information about both indicators of plaque stability $[4,5,31,33,34]$. However, quantification of CNA35 uptake in fluorescence imaging is tedious. Conjugation of CNA35 with e.g. SPECT probes may allow such quantification. Also, conjugation of CNA35 with other (multimodal) contrast agents will enable utilization of imaging modalities such as MRI and PET, allowing both invasive and noninvasive functional and structural imaging. This further establishes the potential of CNA35 as a molecular imaging agent, both of early and late stages of atherosclerosis. However, CNA35/OG488 does not allow for detection of all vulnerable plaques, such as eroded plaques or plaques with mild or moderate calcification (plaque morphologies associated with acute coronary thrombi) [35]. Since vulnerability for plaque rupture potentially is a plural process, unequivocally detection of all vulnerable plaques would require multi-targeted probes. The in vivo labeling characteristics of CNA35/OG488 in kidneys, heart, and liver underline its potential as a molecular agent in other tissues as well. Therefore, CNA35/OG488 may also be a valuable tool for studying diseases such as heart failure, wound healing, or diabetes, where alterations of collagen content or the intactness of endothelium may be of importance.

\section{Acknowledgements}

We thank J.M.E.M. Cosemans from Maastricht University, Department of Biochemistry, for kindly providing the antibodies against type I and III collagen, and M. Breurken and K.N. Krahn (Department of Biomedical Engineering, Eindhoven University of Technology) for the synthesis of CNA35/OG488. The Biorad TPLSM was obtained via a grant (No. 902-16-276) from the Medical Section of the Dutch Scientific Organization (NWO). 
Figures
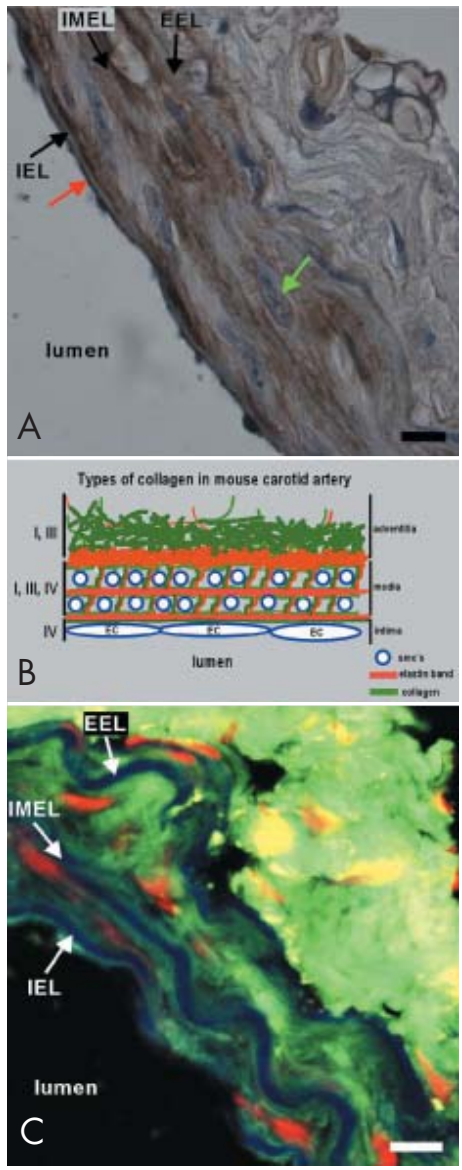

\section{Figure 1}

(A) Example of a fixed histological section of an elastic artery stained for type IV collagen (bar indicates $10 \mu \mathrm{m}$ ). (B) Schematic cross section representing the distribution of type I, III, and IV collagen in wall of a mouse carotid (elastic) artery. (C) Example of fixed histological section of an elastic artery labeled with CNA35/OG488 (bar indicates $10 \mu \mathrm{m})$. Collagen (green) is clearly visible in all tunicae, surrounding the cells (red), the EEL, IMEL, and IEL (blue, auto fluorescence signal). 


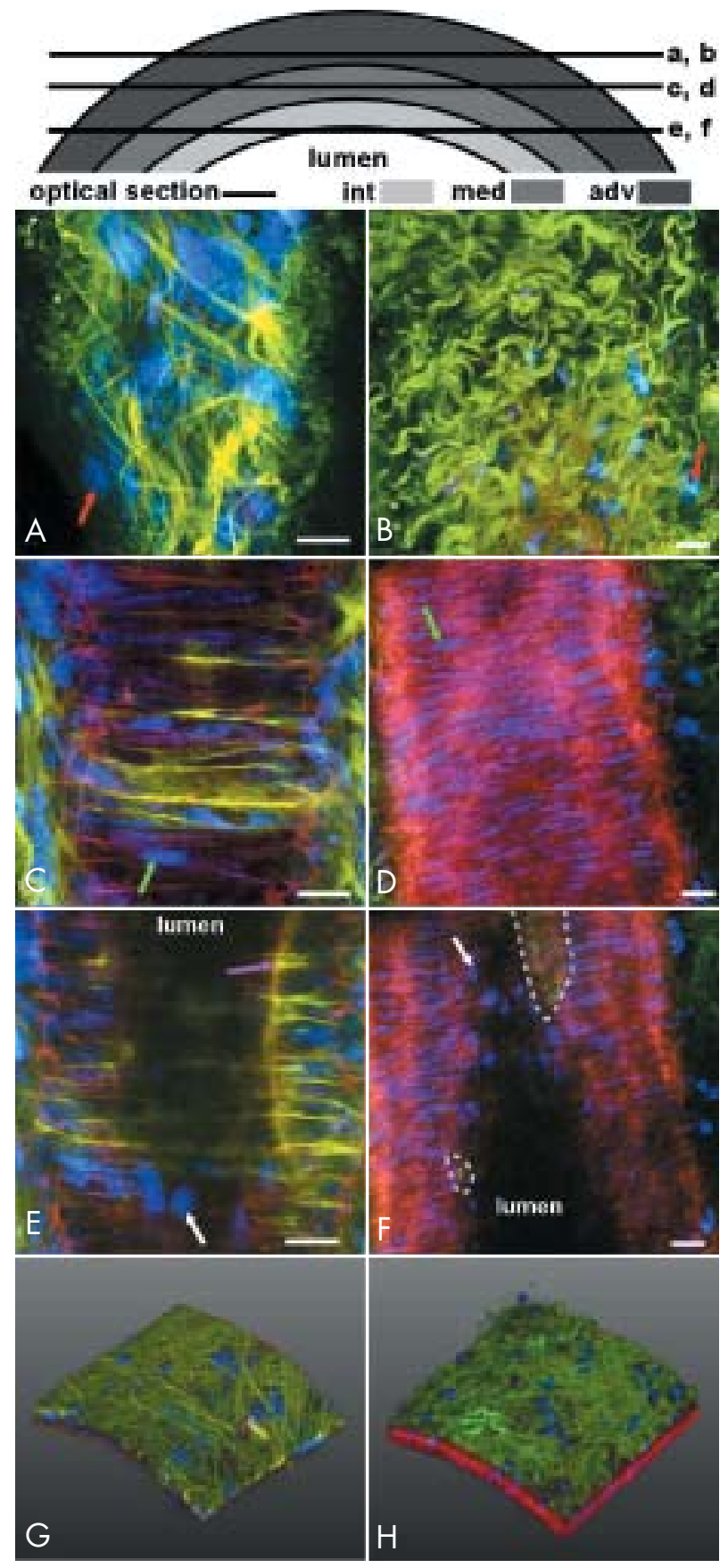

Figure 2

Optical xy-sections of a mounted muscular uterine $(A, C, E)$ and a mounted elastic carotid (B, D, F) artery, ex vivo labeled with CNA35/OG488 (collagen, green), eosin (elastin, red), and SYTO41 (nuclei, blue). Bars indicate $20 \mu \mathrm{m}$; drawing (on top of the optical sections) indicates the positions of the displayed $x y$ sections in the arterial wall. (a) And (B) show the tunica adventitia of a muscular and elastic artery, respectively. Note the differences in collagen structure and content in the tunica adventitia of both arteries. In the tunica media of a muscular artery (C), collagen fibers (green) are clearly distinguishable between the vascular smooth muscle cells (vSMCs, green arrow). Elastin has a mesh-like structure. The tunica media of an elastic artery (D) contains no CNA35/OG488 labeled collagen. The structure of elastin (red) is more compact and completely surrounds $v S M C$ nuclei (green arrow). (E) Represents the tunica intima of a muscular artery with the IEL (red), endothelial cell nuclei (white arrow) and part of the lumen. A strong collagen signal (pink arrow) is visualized near several fenestrae in the IEL. Furthermore, a hazy layer of collagen is present on the luminal site of the IEL. The tunica intima of an elastic artery (F) contains endothelial cells (white arrow), a less fenestrated IEL (red) and lacks the hazy layer of collagen at the luminal site of the IEL. Only few CNA35/OG488 labeled sites (white dotted lines) are present between the endothelial cells and the IEL. (G) and (H) Show 3D-reconstructions of both z-stacks of the muscular and elastic artery, respectively. Note the difference in arterial wall thickness between both arterial types. 

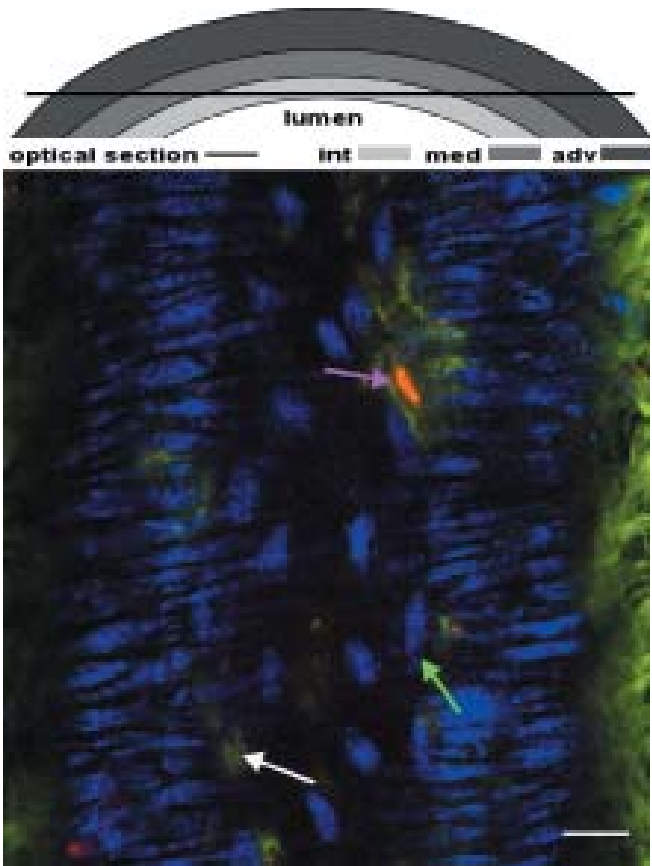

Figure 3

Optical section of an elastic (carotid) artery labeled ex vivo with CNA35/OG488 (collagen, green), SYTO41 (nuclei of cells with an intact membrane, blue) and PI (nuclei of cells with lost membrane integrity, red). Drawing indicates the position of the displayed optical section in the arterial wall. Part of the collagen (green) in the tunica adventitia is visible in the right side of the figure. Furthermore, vSMCs ("cigar-shaped" cell nuclei) located in the tunica media, and endothelial cells (green arrow) in the tunica intima can be clearly identified. CNA35/OG488 uptake in the tunica intima is increased in areas near Pl-positive endothelial cells (pink arrow). However, not all CNA35/OG488 labeled areas in the tunica intima are in close proximity of Pl-positive endothelial cells (white arrow). Bar indicates $20 \mu \mathrm{m}$.
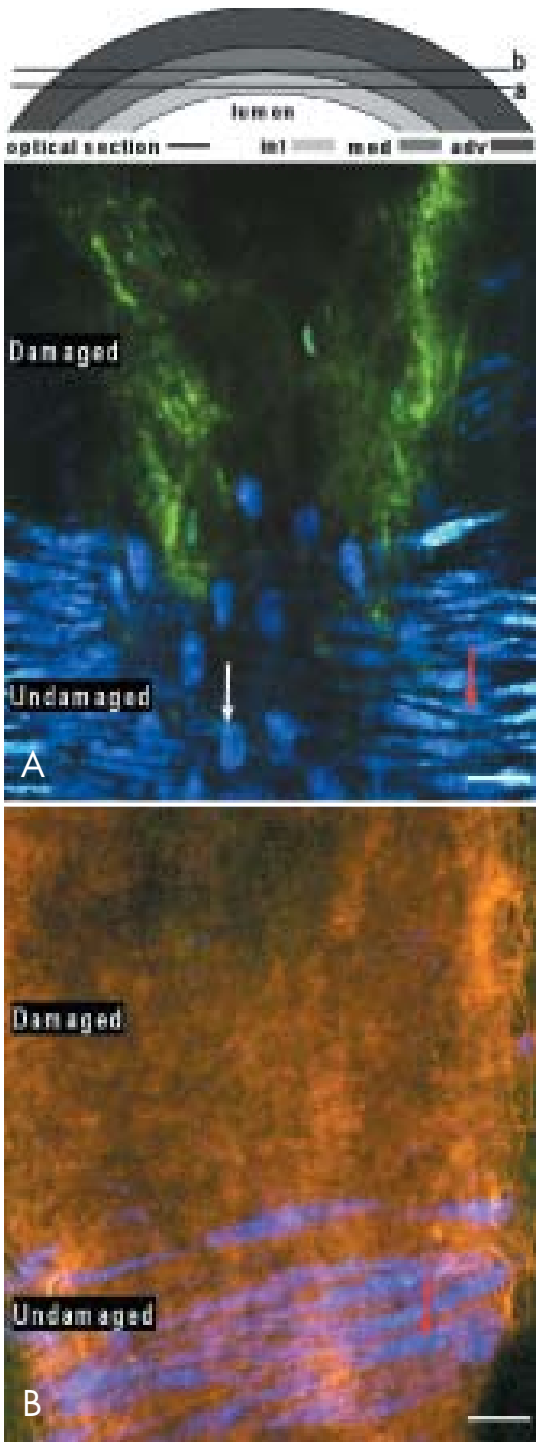

Figure 4

Drawing indicates the position of the displayed optical sections in the artery, bars indicate $20 \mathrm{\mu m}$. (A) optical section of a damaged elastic (carotid) artery labeled with CNA35/OG488 (collagen, green) and SYTO4 1 (cell nuclei, blue). A collagen layer is visible in the tunica intima of the damaged area (B) Comparable optical section of a damaged elastic artery, additionally labeled for elastin (eosin, orange). The IEL (homogeneous orange layer) is unaltered in the damaged area. 


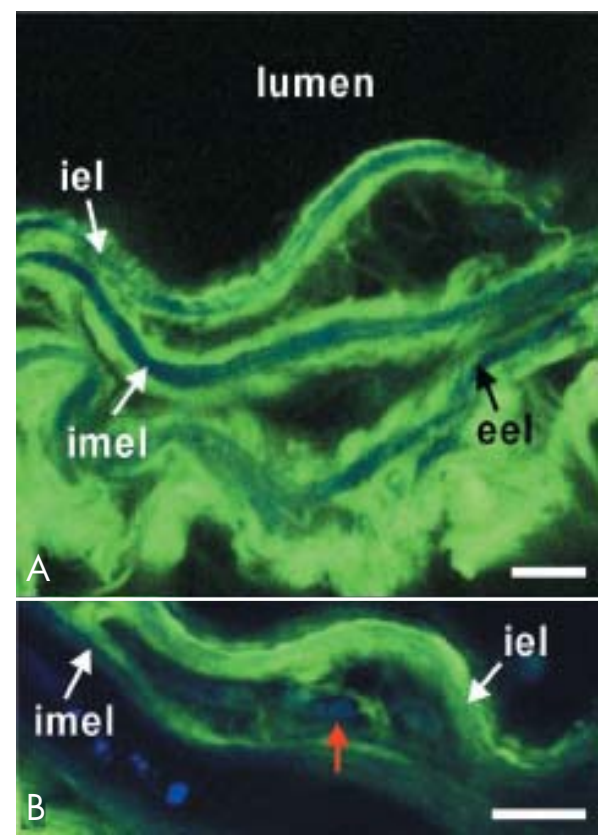

Figure 5

(A) $x y$-section of fresh arterial rings of an elastic artery, labeled with CNA35/OG488 (bar indicates $10 \mu \mathrm{m})$. Collagen (green) is clearly visible in all tunicae, surrounding the EEL, IMEL, and IEL (blue, auto fluorescence signal). (B) Part of a xy-section at slightly higher magnification labeled with CNA35/OG488 (collagen) and SYTO4 1 (cell nuclei, blue) displaying collagen surrounding several vSMCs (red arrow) between the IMEL and IEL in the tunica media (bar indicates $10 \mu \mathrm{m}$ ).
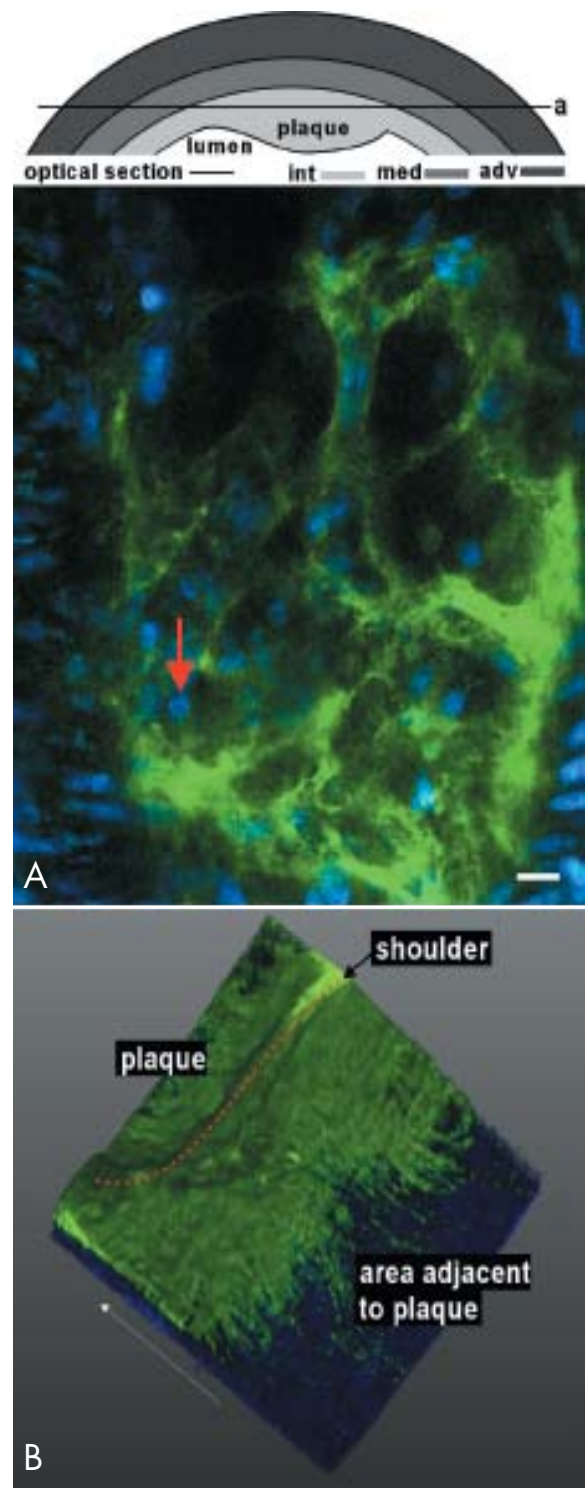

Figure 6

Example of an ex vivo labeled atherosclerotic plaque region in an elastic (carotid) artery stained for collagen (CNA35/OG488, green) and cell nuclei (SYTO41, blue). Bar indicates $10 \mu \mathrm{m}$. (A) Optical section of an atherosclerotic plaque in the intima (see drawing for the position in the arterial wall). Collagen exhibits a network-like pattern (green). Cell nuclei (red arrow) are disorderly arranged between collagen network. (B) 3-dimensional reconstruction of atherosclerotic plaque shoulder (green, indicated by red dotted line). White arrow indicates direction of flow. Arterial wall adjacent to plaque (lower right part of the reconstruction) also contains CNA35/OG488 signal (green). 

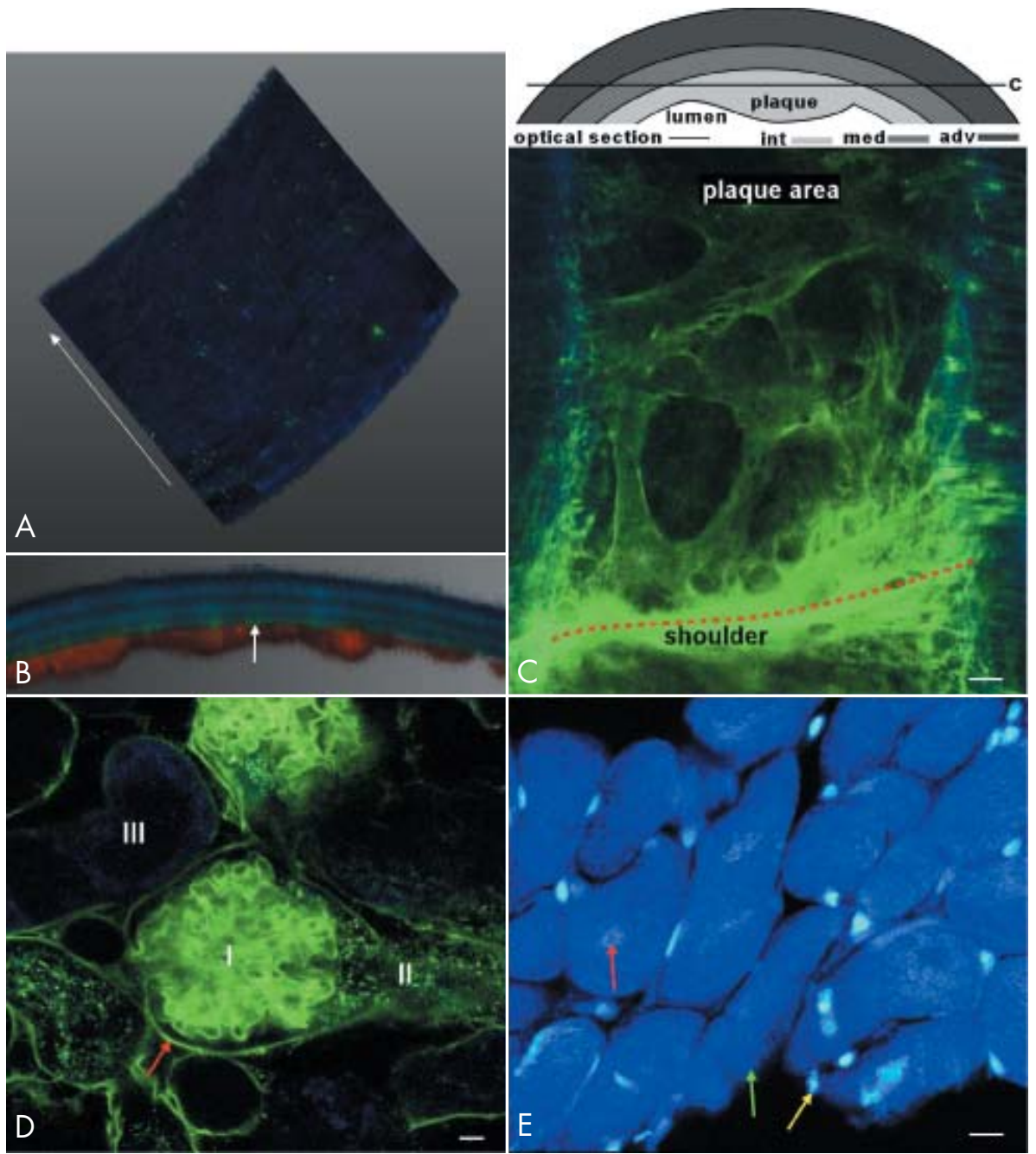

Figure 7

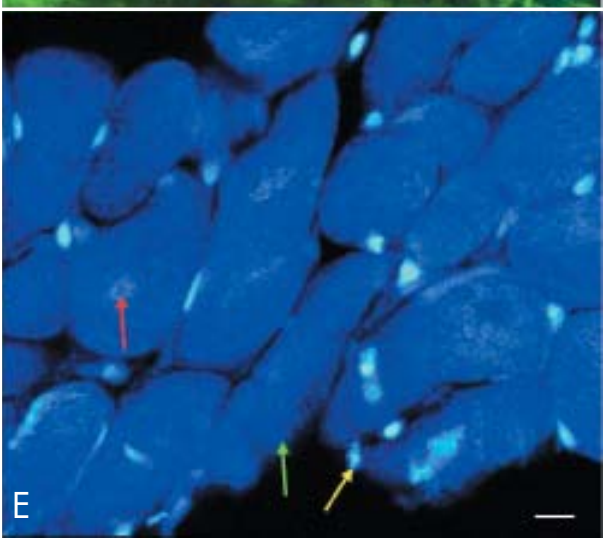

In vivo administration of CNA35/OG488. Bars indicate 10 $\mu \mathrm{m}$. (A) 3D-reconstruction of a healthy carotid artery (white arrow indicates direction of flow). Blue is autofluorescence signal from the three elastic layers (EEL, IMEL, IEL). Only little uptake of CNA35/OG488 (green spots) is detectable. (B) Transversal view of a 3D-reconstruction of the same artery after ex vivo labeling with anti-CD31/PE (endothelial cells, red). The three elastic layers are distinguishable. The weak CNA35/OG488 signal (white arrow) is noticeable between IEL (inner blue layer) and endothelial cells (red). (C) Optical section of an atherosclerotic plaque in a carotid artery of an ApoE-/- mouse. Shoulder of plaque (indicated by red dotted line) is visible in detail and exhibits a strong CNA35/OG488 collagen signal. In the plaque area a collagen network is visible in detail. (D) Optical section of a part of a kidney of a C57BL6/J mouse. The glomerulus (I), Bowmans capsule (red arrow), and proximal tubulus (II) are strongly labeled with CNA35/OG488. Other parts of tubuli (III) exhibit no CNA35/OG488 labeling (blue, autofluorescence). (E) Optical section of a healthy heart of a C57BL6/J mouse after in vivo infusion of CNA35/OG488. After dissection, myocytes (green arrow), nuclei of myocytes (red arrow), and nuclei of the endothelial cells in the vasculature (yellow arrow) were stained with SYTO41, (blue, nuclei and cyłoplasm). 


\section{References}

1. Wilkinson, I.B. and C.M. McEniery, Arterial stiffness, endothelial function and novel pharmacological approaches. Clin Exp Pharmacol Physiol, 2004;31(1 1):795-9.

2. Plenz, G.A., M.C. Deng, H. Robenek, et al., Vascular collagens: spotlight on the role of type VIII collagen in atherogenesis. Atherosclerosis, 2003;166(1):1-11.

3. Rodriguez-Feo, J.A., J.P. Sluijter, D.P. de Kleijn, et al., Modulation of collagen turnover in cardiovascular disease. Curr Pharm Des, 2005;11(19):2501-14.

4. Heeneman, S., J.P. Cleutjens, B.C. Faber, et al., The dynamic extracellular matrix: intervention strategies during heart failure and atherosclerosis. J Pathol, 2003;200(4):516-25.

5. Katsuda, S. and T. Kaji, Atherosclerosis and extracellular matrix. J Atheroscler Thromb, 2003;10(5):267-74.

6. Gaballa, M.A., C.T. Jacob, T.E. Raya, et al., Large artery remodeling during aging: biaxial passive and active stiffness. Hypertension, 1998;32(3):437-43.

7. McEniery, C.M., Novel therapeutic strategies for reducing arterial stiffness. Br J Pharmacol, 2006; 148(7):881-3.

8. Briones, A.M., F.E. Xavier, S.M. Arribas, et al., Alterations in structure and mechanics of resistance arteries from ouabain-induced hypertensive rats. Am J Physiol Heart Circ Physiol, 2006;291(1):H193-201.

9. Vranes, D., M.E. Cooper, and R.J. Dilley, Cellular mechanisms of diabetic vascular hypertrophy. Microvasc Res, 1999;57(1):8-18.

10. Megens, R.T., S. Reitsma, P.H. Schiffers, et al., Two-photon microscopy of vital murine elastic and muscular arteries. Combined structural and functional imaging with subcellular resolution. J Vasc Res, 2007;44(2):87-98.

11. Martinez-Lemus, L.A., M.A. Hill, S.S. Bolz, et al., Acute mechanoadaptation of vascular smooth muscle cells in response to continuous arteriolar vasoconstriction: implications for functional remodeling. Faseb J, 2004;18(6):708-10.

12. van Zandvoort, M., W. Engels, K. Douma, et al., Two-photon microscopy for imaging of the (atherosclerotic) vascular wall: a proof of concept study. J Vasc Res, 2004;41(1):54-63.

13. Boulesteix, T., A.M. Pena, N. Pages, et al., Micrometer scale Ex Vivo multiphoton imaging of unstained arterial wall structure. Cytometry A, 2006;69(1):20-6.

14. Ferrara, D.E., D. Weiss, P.H. Carnell, et al., Quantitative 3D fluorescence technique for the analysis of en face preparations of arterial walls using quantum dot nanocrystals and twophoton excitation laser scanning microscopy. Am J Physiol Regul Integr Comp Physiol, 2006;290(1):R $114-23$.

15. Campagnola, P.J., A.C. Millard, M. Terasaki, et al., Three-dimensional high-resolution secondharmonic generation imaging of endogenous structural proteins in biological tissues. Biophys J, 2002;82(1 Pt 1):493-508.

16. Zoumi, A., A. Yeh, and B.J. Tromberg, Imaging cells and extracellular matrix in vivo by using second-harmonic generation and two-photon excited fluorescence. Proc Natl Acad Sci U S A, 2002;99(17): $11014-9$. 
17. Zoumi, A., X. Lu, G.S. Kassab, et al., Imaging coronary artery microstructure using secondharmonic and two-photon fluorescence microscopy. Biophys J, 2004;87(4):2778-86.

18. Krahn, K.N., C.V. Bouten, S. van Tuijl, et al., Fluorescently labeled collagen binding proteins allow specific visualization of collagen in tissues and live cell culture. Anal Biochem, 2006;350(2):177-85.

19. Patti, J.M., J.O. Boles, and M. Hook, Identification and biochemical characterization of the ligand binding domain of the collagen adhesin from Staphylococcus aureus. Biochemistry, 1993;32(42): 1 1428-35.

20. Xu, Y., J.M. Rivas, E.L. Brown, et al., Virulence potential of the staphylococcal adhesin CNA in experimental arthritis is determined by its affinity for collagen. J Infect Dis, 2004; 189(12):2323-33.

21. Rich, R.L., C.C. Deivanayagam, R.T. Owens, et al., Trench-shaped binding sites promote multiple classes of interactions between collagen and the adherence receptors, alpha(1)beta(1) integrin and Staphylococcus aureus cna MSCRAMM. J Biol Chem, 1999;274(35):24906-13.

22. Zong, Y., Y. Xu, X. Liang, et al., A 'Collagen Hug' model for Staphylococcus aureus CNA binding to collagen. Embo J, 2005;24(24):4224-36.

23. Junqueira, L.C. and J. Carneiro, Basic Histology. Eleventh edition ed. 2005, New York: McGraw-Hill Medical Publishing Division.

24. Hilgers, R.H., S. Bergaya, P.M. Schiffers, et al., Uterine Artery Structural and Functional Changes During Pregnancy in Tissue Kallikrein-Deficient Mice. Arterioscler Thromb Vasc Biol, 2003;23(10):1826-1832.

25. Havenith, M.G., J.P. Cleutiens, C. Beek, et al., Human specific anti-type IV collagen monoclonal antibodies, characterization and immunohistochemical application. Histochemistry, 1987;87(2):123-8.

26. Konig, K., Multiphoton microscopy in life sciences. J Microsc, 2000;200 ( P+ 2):83-104.

27. Cosemans, J.M., M.J. Kuijpers, C. Lecut, et al., Contribution of platelet glycoprotein VI to the thrombogenic effect of collagens in fibrous atherosclerotic lesions. Atherosclerosis, 2005; $181(1): 19-27$.

28. Ghitescu, L. and M. Robert, Diversity in unity: the biochemical composition of the endothelial cell surface varies between the vascular beds. Microsc Res Tech, 2002;57(5):381-9.

29. Guyton, A.C. and J.E. Hall, Textbook of Medical Physiology. Ninth edition ed. 1996: W.B.Saunders company. 1148.

30. Spina, M., A. Friso, A.R. Ewins, et al., Physicochemical properties of arterial elastin and its associated glycoproteins. Biopolymers, 1999;49(3):255-65.

31. Ross, R., Atherosclerosis-an inflammatory disease. N Engl J Med, 1999;340(2):1 15-26.

32. Stary, H.C., A.B. Chandler, R.E. Dinsmore, et al., A definition of advanced types of atherosclerotic lesions and a histological classification of atherosclerosis. A report from the Committee on Vascular Lesions of the Council on Arteriosclerosis, American Heart Association. Circulation, 1995;92(5):1355-74. 
33. Lusis, A.J., Atherosclerosis. Nature, 2000;407(6801):233-41.

34. Lutgens, E., K.B. Cleutjens, S. Heeneman, et al., Both early and delayed anti-CD4OL antibody treatment induces a stable plaque phenotype. Proc Natl Acad Sci U S A, 2000;97(13):7464-9.

35. Kolodgie, F.D., A.P. Burke, A. Farb, et al., The thin-cap fibroatheroma: a type of vulnerable plaque: the major precursor lesion to acute coronary syndromes. Curr Opin Cardiol, $2001 ; 16(5): 285-92$. 
Chapter 4 


\section{Chapter 5}

\section{Imaging Collagen and Inflammatory Cells in Atherosclerotic Plaques in Vital Carotid Arteries using Two-Photon Microscopy}

submitted

Remco T.A. Megens a; Mirjam G.A. oude Egbrink b; Maarten Merkx c; Dick W. Slaaf ${ }^{a, c}$; Marc A.M.J. van Zandvoort '.

Departments of ${ }^{\circ B i o m e d i c a l ~ E n g i n e e r i n g ~ a n d ~ ' P h y s i o l o g y, ~ C a r d i o v a s c u l a r ~ R e s e a r c h ~ I n s t i t u t e ~}$ Maastricht, Maastricht University, Maastricht, the Netherlands.

'Department of Biomedical Engineering, Technical University Eindhoven, P.O.Box 513, 5600MB, Eindhoven, the Netherlands. 


\section{Abstract}

Objective: atherogenesis involves structural and cellular changes in the vessel wall of large arteries. We used two-photon laser scanning microscopy (TPLSM) to visualize atherogenesis in intact, viable carotid arteries of mice.

Methods: isolated carotid arteries of $\mathrm{Apo} \%$ mice, aged 15 or 21 weeks, after 7 and 13 weeks on a western diet, were mounted in a perfusion chamber, pressurized, and labeled with specific fluorescent markers for cell nuclei, inflammatory cells, collagen, and lipids. Data were compared with C57BL6/J control mice fed a chow diet.

Results: control vessels had intact endothelium without adhering blood cells or significant intimal collagen labeling. In contrast, in $\mathrm{ApoE}^{\%}$ mice already at 15 weeks inflammatory cells adhered to the endothelium and increased labeling of collagen was observed in the tunica intima both at lesion-prone and nonlesion-prone sites, indicating endothelium activation. In plaques, located exclusively in carotid bifurcations of ApoE $/$ mice, internalized inflammatory cell density increased with age and plaque progression. Interestingly, this was observed both in the tunicae adventitia and intima, while in the tunica media no inflammatory cells were detected at both ages. In the whole plaque, aging or plaque progression did not alter the direct relation between inflammatory cells and collagen. However, within the fibrous caps specifically, direct contact between inflammatory cells and collagen increased with age.

Conclusion: this study on viable and structurally intact arteries provides new and detailed information regarding the complex relationship between inflammatory cells and collagen in fibrous caps and whole plaques during atherogenesis and suggests involvement of the tunica adventitia in atherogenesis. 


\section{Introduction}

Vulnerable plaques are the major cause of acute clinical complications of atherosclerosis [1]. Vulnerable plaques can rupture and cause a thrombotic reaction that can occlude the (downstream) artery which may result in stroke or myocardial infarction [2]. Major determinants of vulnerability of human atherosclerotic plaques are extracellular matrix content and extent of inflammation [1,3,4]. Stable plaques have a large collagen content and are mostly surrounded by a thick, collagen-rich fibrous cap [5]. Loss of collagen reduces tensile strength of the fibrous cap and increases probability of rupture $[6,7]$. Atherosclerotic plaques also contain inflammatory cells such as macrophages that are known to produce enzymes like matrix metalloproteinases that break down extracellular matrix proteins such as collagen and inhibit collagen synthesis by smooth muscle cells $[4,8,9]$. It is generally accepted that as a result, stability of atherosclerotic plaques may be affected $[2,10]$. Indeed, histological studies revealed that collagen-poor and inflammation-rich plaques are more likely to rupture [10].

Mouse models of atherosclerosis, such as apolipoprotein E knockout mice $(A p o E)$, are advantageous for studying the (chronic) process of atherogenesis. However, most of present knowledge on atherosclerosis in such models is still obtained by histological studies using sliced, fixated non-viable arteries. Only little is known of the 3-dimensional organization of the arterial wall in intact and viable large arteries containing atherosclerotic plaques. Such data will provide new insight into plaque structure and the relation and mutual influence between inflammatory cells and collagen in plaques throughout their development.

Two-photon laser-scanning microscopy (TPLSM) is a technique combining large penetration depth with subcellular resolution, also in deeper layers [11]. Its good optical sectioning properties, good sensitivity, and high (fluorescent) contrast, enabled 3-dimensional imaging at subcellular level of large mouse arteries mounted and pressurized in a perfusion chamber, maintaining their physiological structure, viability, and functionality for several hours $[12,13]$ or in a non-mounted setting [14-17].

In this study TPLSM was applied on mounted arteries labeled with specific, vital fluorescent markers for collagen [13, 18], inflammatory cells, cell nuclei, and lipids to gain insight in the distribution of collagen and its association with inflammatory cells during atherogenesis. 


\section{Materials and Methods}

\section{Animals}

Experiments and procedures were approved by the local ethics committee on use of laboratory animals. Experimental groups consisted of C57BL6/J control mice (14-16 weeks $(n=7) ; 20-22$ weeks $(n=7))$, and atherosclerosis-prone ApoE ${ }^{-1}$ mice (14-16 weeks ( $\left.n=7\right) ; 20-22$ weeks $\left.(n=6)\right)$. Mice were obtained from Charles River, Maastricht, the Netherlands. ApoE $\%$ mice were fed a western diet starting at the age of 8 weeks (WD4021.06; Hope Farms, Woerden, the Netherlands), while C57BL6/J controls were fed a chow diet (SSNIFF; Bioservices, Uden, the Netherlands). Mice were euthanized by a mixture of $\mathrm{CO}_{2}$ and $\mathrm{O}_{2}$, after which carotid artery segments (common part and bifurcation) were excised.

\section{Tissue preparation and staining}

Excised carotid arteries, including bifurcation, were mounted in a perfusion chamber [12, 13]. Side branches were ligated. The system was filled with Hanks Balanced Salt Solution (HBSS, $\mathrm{pH} 7.4$ ) containing in mmol/l: $\mathrm{NaCl}$ 144, HEPES 14.9, glucose 5.5, $\mathrm{KCl} 4.7, \mathrm{CaCl} 22.5, \mathrm{KH}_{2} \mathrm{PO}_{4} 1.2$, and $\mathrm{MgSO}_{4}$ 1.2. On average, intraluminal pressure was $50 \mathrm{mmHg}$ (dependent on maximum pressure ligations could endure). All fluorescent markers were dissolved in HBSS and administered both intra- and extraluminally. Incubation started 30 minutes prior to start of image acquisition. Probes remained present during image acquisition, since this did not alter their labeling characteristics, viability of arteries, nor reduce image quality.

DNA/RNA markers SYTO41 ( $\left.\lambda_{\text {max emission }}=480 \mathrm{~nm}\right)$ and SYTO13 $\left(\lambda_{\text {max emission }}=520 \mathrm{~nm}\right)$ were used for labeling cell nuclei (final concentration $2.0 \mathrm{\mu mol} / \mathrm{l}$; Invitrogen, Breda, the Netherlands). Phycoerythrin (PE, $\lambda_{\text {max }}$ emission $=570 \mathrm{~nm}$ ) conjugated rat anti-mouse CD1 1 b (MAC-1 $\alpha$ or CD18) was applied as marker for inflammatory cells such as mature macrophages [19], monocytes, and granulocytes (final concentration $8 \mathrm{\mu g} / \mathrm{ml}$; BD Biosciences, Alphen aan den Rijn, the Netherlands). Oregon Green 488 conjugated CNA35 (CNA35/OG488, $\lambda_{\text {max emission }}=510 \mathrm{~nm}$ ) was used for staining of collagen $[13,18]$ (final concentration $1.5 \mu \mathrm{mol} / \mathrm{l}$ ).

Two mounted and visualized arteries (ApoE $\%$ mice, 21 weeks) were also studied for lipid content. They were incubated overnight at $7^{\circ} \mathrm{C}$ with the lipid marker oil red $\bigcirc$ (ORO, $\lambda_{\text {max emission }}=560$ nm; Fluka Chemie, Buchs, Switzerland) dissolved in $3.7 \%$ formaldehyde solution (Merck, Darmstadt, Germany) to final concentration of $12 \mu \mathrm{mol} / \mathrm{I}[14,20]$. ORO staining resulted in significant reduction of fluorescence of previously applied labels and loss of cell viability. Furthermore, arterial rings (approximately $1 \mathrm{~mm}$ long) from 3 
isolated and previously mounted arteries (ApoE $\%$ mice, 21 weeks) were labeled with ORO and transversally imaged for visualization of lipid content of plaques. After overnight incubation, arterial rings were labeled with SYTO13 (2.0 pmol/l, 30 minutes) and rings were casted in agarose gel $11.5 \%$ dissolved in HBSS, Gibco BRL 15510-027; Invitrogen Breda, the Netherlands) as previously described [14].

\section{Imaging}

The TPLSM [12, 14] consisted of a Biorad 2100MP TPLSM (Biorad, Hemel Hempstead, UK) with a pulsed (80-100 fs) and mode-locked (800 nm) Ti:sapphire laser as excitation source (Spectra Physics Tsunami, Mountain View, USA) connected to a Nikon E600FN microscope $(60 \mathrm{X}, \mathrm{NA}=1.0$ water dipping objective; Nikon Corporation, Tokyo, Japan). Damage to the sample was avoided by keeping excitation laser power as low as possible [21]. On average, laserpower of the excitation light was set at $15 \mathrm{~mW}$ for imaging in the tunica adventitia, $22 \mathrm{~mW}$ for imaging in the tunica media, and $30 \mathrm{~mW}$ for imaging in the tunica intima. In atherosclerotic lesions, laserpower was maximized to $60 \mathrm{~mW}$ for imaging of plaque cores. Imaging rate was either $0.1 \mathrm{~Hz}$ with a pixel dwell time of $39 \mu \mathrm{s}$, or $0.3 \mathrm{~Hz}$ with a pixel dwell time of 0.12 us combined with Kalman filtering for noise reduction ( $n=3$ cycles). Fluorescent signals were detected by three photomultiplier tubes (PMTs): SYTO41, 460-480 nm (PMT I); CNA35/OG488, 520-550 nm (PMT II); CD1 1b/PE, 570-610 nm (PMT III); ORO, 540-610 nm, (PMT III). From each PMT, separate images of 512 * 512 pixels were obtained, saved, and combined into a single RGB-image. Filter setting for each PMT was tuned for minimal bleedthrough of fluorescent signal of the markers. Furthermore, PMT gain was set to $100 \%$ in order to limit the required laserpowers, while obtaining maximal signal intensity in deeper layers and atherosclerotic lesions of the arterial vessel wall.

Stacks of optical sections were collected for 3D-reconstructions to screen for plaques in the entire mounted vessel; staining patterns in plaques differed from those of healthy carotid arteries. Plaque detection was checked using bright field microscopy where plaques showed up as dark and less translucent regions on a bright background.

Image analysis was performed using Image-Pro Plus 6.0 and 3D-reconstructor 5.1 software package (Media Cybernetics Inc., Silver spring, USA).

\section{Relationship between CNA35/OG488 and CD1 1b/PE-positive cells}

To explore the relationship between collagen and inflammatory cells in initial, mild, and advanced plaques [22], we assessed (a) number of inflammatory 
(CD 1 1b/PE-positive) cells per $(100 \mu \mathrm{m})^{3}$ plaque volume and (b) contact ratio, being the relative number of inflammatory cells in contact with collagen. Plaque volumes were estimated using optical section thickness and surface area, excluding areas without fluorescent signal. The relation between collagen (CNA35/OG488 labeled structures) and inflammatory (CD1 1b/PE-positive) cells in fibrous caps of mild and advanced lesions of ApoE $\%$ mice of both ages $(n=14$, only plaques with clearly visible fibrous cap) was scored: $(0)<33 \%$; (1) $33-67 \%$; (2) > $67 \%$ of inflammatory cells in contact with CNA35/OG488 labeled collagen.

\section{Cells in tunica adventitia}

Inflammatory (CD11b/PE-positive) cells and CD11b/PE-negative cells were counted in the tunica adventitia of atherosclerotic $(n=9)$ and control $(n=12)$ areas in the arterial wall. The ratio of inflammatory cells was assessed per total number of cells per $(100 \mu \mathrm{m})^{3}$ volume.

\section{Histology}

Histological sections were prepared to validate penetration depth of TPLSM in plaques in mounted arteries. Subsequent to imaging of mounted arteries ex vivo, arteries (two C57BL6/J mice; two ApoE $\%$; all 21 weeks) were perfusionfixated and processed to $5 \mathrm{\mu m}$ thick histological sections and stained for collagen with Picrosirius Red [23] (0.1 \%, Klinipath, Duiven, the Netherlands). Histological sections were imaged using a Leica DM 5000B microscope $160 \mathrm{X}$ oil objective; $N A=1.4$ ) and a Leica DC300FX digital camera (Leica Microsystems $\mathrm{GmbH}$, Wetzlar, Germany).

\section{Statistics}

Data are presented as medians $(M)$ with interquartile ranges (IQR): $M$ [IQR]. Results were tested for significance using the Mann-Whitney test. A value of $\mathrm{p}<0.05$ was considered statistically significant. SPSS 13.0 software package (SPSS Inc., Chicago, USA) was used for statistical analysis. 


\section{Results}

\section{Carotid arteries of C57BL6/J mice}

Carotid arteries of C57BL6/J mice contained no lesions (table 1); vessel wall structure was normal [12]. The tunica adventitia consisted of cells (SYTO41) embedded in collagen (CNA35/OG488). The vSMCs in the tunica media were homogeneously distributed and arranged perpendicular to flow direction. Endothelial cell nuclei were homogeneously distributed covering the tunica intima (fig. 1A). No inflammatory (CD1 1 b/PE-positive) cells were present in or adhering to the arterial wall. Labeled collagen was only sparsely observed in the tunica intima and not at all in the tunica media (see also [13]). Hence, the endothelial cells appear non-activated and non-permeable for small molecules. The barrier function of internal elastic layer (IEL) and external elastic layer (EEL) appear intact, as deduced from the non-permeability for the collagen probe [13].

Table 1 Mouse characteristics and presence of atherosclerotic lesions in C57BL6/J and ApoE mice of 15 and 21 weeks

\begin{tabular}{lllll}
\hline & C57 (15wks) & C57 (21wks) & ApoE $\%$ (15wks) & ApoE $\%$ (21wks) \\
\hline \# mice (\#carotids) & $7(13)$ & $7(12)$ & $7(13)$ & $6(11)$ \\
$\begin{array}{l}\text { weight (g) } \\
\text { (avg } \pm \text { SD) }\end{array}$ & $26.5 \pm 1.9$ & $29.2 \pm 2$ & $27.8 \pm 2.6$ & $30.2 \pm 2.4$ \\
$\begin{array}{l}\text { \# mice with plaque } \\
\text { (\#carotids with plaques) }\end{array}$ & $0(0)$ & $0(0)$ & $5(9)$ & $6(11)$ \\
$\begin{array}{l}\text { Total \# plaques in } \\
\text { analyzed segments }\end{array}$ & - & & 12 & 21 \\
$\begin{array}{l}\text { class 1 plaques (\%) } \\
\text { class 2 plaques (\%) }\end{array}$ & - & - & $7(58)$ & $4(19)$ \\
class 3 plaques (\%) & - & - & $5(42)$ & $5(24)$ \\
\hline
\end{tabular}

\section{Common carotid arteries of ApoE ${ }^{-1}$ mice}

In the common carotid artery of both 15 and 21 weeks old ApoE $\%$ mice no atherosclerotic lesions were found; tunica adventitia and media were undisturbed. However, a thin sheet of collagen (fig. 1B) was observed between endothelium and IEL of the tunica intima at both ages. In addition, inflammatory cells adhering to the luminal side of the endothelium were found in mice of both ages (fig. 1C). Number of cells adhering to the endothelium increased with age from 0.2 [0-0.6] cells per $(100 \mu \mathrm{m})^{2}$ at 15 weeks to 0.6 [0.3-1.2] at 21 weeks $(p<0.05)$. No inflammatory cells were present inside the arterial wall. These 
data suggests that endothelial cells were activated and permeable for the collagen probe, while IEL and EEL were still intact.

\section{Morphology of lesion development in the carotid bifurcation of $\mathrm{ApoE}$ - mice} In the carotid bifurcation of most $\mathrm{ApoE}^{\%}$ mice of both 15 and 21 weeks, atherosclerotic lesions were detected exhibiting strong collagen and cellular staining. In all phases of lesion development, a thin sheet of collagen was present in the tunica intima adjacent to the plaque area between endothelial cells and IEL. This indicates increased CNA35/OG488 permeability of endothelial cells in these regions, and possibly reflects increased collagen content in this layer. In young mice, plaques were of initial (class 1) or mild (class 2) type. No advanced (class 3) plaques were found. In contrast, in mice of 21 weeks the majority of plaques were class 3 (table 1).

In the initial phase of plaque development (class 1, see fig. 1D), inflammatory cells were accumulated in the tunica intima underneath the endothelial cells. Furthermore, collagen in the lesions formed a thin sheet or local spots. In the tunica media underneath these initial plaques, orientation and distribution of vSMCs was unaltered. No collagen or inflammatory cells were observed.

During further lesion progression, mild plaques developed (class 2). First signs of a fibrous collagen-rich cap structure could be observed at 15 weeks (fig. 2A, class 2). In addition, infiltrated inflammatory cells were present in the tunica intima. In class 2 plaques at 21 weeks (fig. 2B) the area of arterial wall affected by the atherosclerotic process appeared to be larger. Often, collagen surrounded areas containing a combination of inflammatory cells (e.g. foam cells) or poorly fluorescent areas without visible structures (i.e., lesion cores) (fig. 2B). The tunica media underneath class 2 plaques was free of inflammatory cells. Furthermore, vSMCs were homogeneously distributed and their orientation seemed unaffected. Collagen between vSMCs was not labeled.

Progression of a substantial number of lesions towards an advanced phase of atherosclerosis was only observed in 21 weeks aged $\mathrm{ApoE}^{-1}$ mice (class 3; table 1). A number of advanced plaques (fig. 3A-D) consisted of strongly labeled collagen with a network-like appearance. Inside the collagen network, large lesion core(s) were present which contained only little fluorescent signal. Infiltrated inflammatory cells were predominantly positioned at the edges between lesion cores and collagen network (fig.3A). Shoulders of class 3 plaques often contained strongly labeled collagen (fig. 3B). In some shoulder 
areas of these plaques, strongly labeled collagen seemed to protrude beyond the edges of the lesion towards the healthy arterial wall (fig. 3B).

Other class 3 plaques manifested as interconnected groups of smaller lesions with less evident plaque borders (fig. 3C). Such lesions often consisted of many small lesion cores with weak fluorescent signal surrounded by large amounts of labeled collagen with a less defined network-like appearance. Many inflammatory cells of various size and shape were observed throughout the lesion.

Application of a lipid stain (ORO) in mounted arteries with class 3 lesions revealed that the dark lesion cores inside plaques had a lipid-rich content (fig. 4A). Imaging of the content of the cores in fresh (but not viable) arterial rings labeled with ORO (fig. 4B-C) demonstrated that part of the lipids was situated inside (foam) cells as small droplets.

In some of the large class 3 lesions the fluorescent signal intensity drastically decreased towards the lumen (fig. 5A). As a result, we were unable to visualize the complete lesion area in depth (fig. 5B); no fibrous cap or endothelial cell lining was visible using TPLSM. However, histological longitudinal sections stained for collagen revealed that such lesions do contain fibrous caps with endothelial cells (fig. 5C).

In all class 3 plaques the tunica media was affected. Although no inflammatory cells were detected in this layer, the $\mathrm{VSMC}$ layer was disrupted and orientation of vSMCs was altered (fig. 3D). Sometimes, collagen between vSMCs in these plaques was labeled, indicative of local disruption of IEL. The tunica adventitia of carotid arteries in both non-plaque regions (in $\mathrm{ApoE}^{-}$and wild type mice; fig. 6A) and plaque regions (in $\mathrm{ApoE}^{\%}$ mice; fig. $6 \mathrm{~B}$ ) contained large amounts of collagen with a typical wave-like appearance. No apparent changes in collagen structure were found between plaque regions and non-plaque regions. Furthermore, the tunica adventitia contained numerous inflammatory cells and non-inflammatory cells in both plaque and non-plaque regions.

\section{Inflammatory cell density}

During lesion development, inflammatory cell density in plaques increased significantly with age $(p<0.05)$ and with plaque progression $(p<0.01$; table 2$)$. The tunica media in plaque regions did not contain any inflammatory cells. In the tunica adventitia, total cell density tended to be higher in plaque regions than in non-plaque regions in mice of both ages (23.6) $\{31.1-17.8\}$ cells per $(100 \mu \mathrm{m})^{3}$ vs. $17.6\{23.6-13.3\}$ ( $\left.p=0.07\right)$. The relative number of inflammatory cells in the tunica adventitia in mice of both ages was increased in plaque 
regions $(0.58\{0.63-0.51\})$ as compared to non-plaque regions $(0.24\{0.36-$ $0.18\} ; p<0.001)$.

\section{Relation between collagen and inflammatory cells in developing plaques}

In plaques as a whole, the percentage of inflammatory cells in direct contact with collagen did not change significantly with age or plaque progression (table 2). In fibrous caps, the median score of the association between collagen and inflammatory cells was significantly higher at 21 weeks (1.0 \{2-1\}) than at 15 weeks $(0.5\{1-0\} ; p<0.05)$. Note that fibrous caps were present only in class 2 and class 3 plaques. Moreover, class 3 plaques were only present in 21 weeks aged $\mathrm{ApoE} \%$.

Table 2 Intraplaque inflammatory cell density and association between inflammatory cells and collagen (medians with interquartile ranges).

\begin{tabular}{llllll}
\hline & $\begin{array}{l}\text { ApoE } / \\
15 w k s\end{array}$ & $\begin{array}{l}\text { ApoE }{ }^{-} \\
21 w k s\end{array}$ & $\begin{array}{l}\text { Plaques } \\
\text { class } 1\end{array}$ & $\begin{array}{l}\text { Plaques } \\
\text { class } 2\end{array}$ & $\begin{array}{l}\text { Plaques } \\
\text { class 3 }\end{array}$ \\
\hline $\begin{array}{l}\text { \# inflammatory } \\
\text { cells } /(100 \mu \mathrm{m})^{3}\end{array}$ & $15[11-18.7]$ & $20[14.5-35]^{*}$ & $12[3-17]$ & $18[11-24.3]^{\S}$ & $27.5[19.5-41.3]^{\S \dagger}$ \\
Ratio & 0.64 & 0.56 & 0.64 & 0.57 & 0.52 \\
& {$[0.55-0.67]$} & {$[0.46-0.64]$} & {$[0.63-0.67]$} & {$[0.51-0.65]$} & {$[0.39-0.64]^{\varepsilon}$}
\end{tabular}

Ratio is \# inflammatory cells with collagen contact divided by total \# inflammatory cells. " $p<0.05$ compared with 15 weeks ApoE $\%$; $p<0.05$ compared with class 1 plaques; ${ }^{\dagger} p<0.05$ compared with class 2 plaques. ${ }^{\varepsilon} p<0.05$ compared with class 1 plaques.

\section{Discussion}

Intact, viable, and mounted carotid arteries of control C57BL6/J and atherosclerotic ApoE $\%$ mice were visualized in three dimensions at subcellular level. Control vessels had intact endothelium without adhering blood cells. In ApoE $\%$ mice, the endothelium was activated, even at non-atherosclerosis prone sites, as indicated by the presence of adhering inflammatory cells and the increase of labeled collagen in the tunica intima. In plaques, located exclusively in the carotid bifurcation of ApoE $\%$ mice, inflammatory cell density increased with age and plaque progression. In fibrous caps, direct contact between inflammatory cells and collagen increased with age. However, in plaques as a whole, aging or plaque progression did not alter the direct relation between inflammatory cells and collagen. The increased number of inflammatory cells in the tunica adventitia of lesions is remarkable since inflammatory cells were absent in the tunica media of these lesions. 
In contrast to control mice, endothelial cells appeared to be activated in carotid arteries of $\mathrm{ApoE} \%$ mice, both in and outside the lesion area, and also in the common carotid artery. It is unlikely that this activation resulted from the mounting procedure since it was not observed in similarly treated control vessels.

The adhering cells (that remained present even after intraluminal flushing) were most probably monocytes since adhesion of these cells is an early response to hypercholesterolemia [7]. Indeed, their morphology as detected in this study (fig. 1) corresponds to that of monocytes (cells with large nucleus) [24]. The observation that the number of adhering cells was higher at 21 than at 15 weeks implies that the level of endothelial activation depends on increased duration of western diet and/or age. Besides increased cell adhesion, increased collagen labeling was observed in the tunica intima of common carotid arteries in $\mathrm{ApoE}^{\%}$ mice at both ages. This is in line with previous findings on properties of the collagen label CNA35/OG488 in (atherosclerotic) arteries [13], implying that this is most probably caused by increased permeability of the endothelium as a consequence of factors like hypercholesteremia [25]. However, it cannot be excluded that intimal collagen content itself increased as well. The combination of increased collagen labeling in the tunica intima and adhesion of inflammatory cells to the endothelium at sites where no lesions are formed or present, suggests that all endothelial cells in carotid arteries of $\mathrm{ApoE}^{-1}$ mice on a western diet are activated.

Inflammatory cell density in plaques (carotid bifurcation) significantly increased with age and plaque progression. Simultaneously, a prominent network-like collagen structure inside the plaques and a collagen rich fibrous cap developed, in line with literature stating that extracellular matrix components accumulate in the atherosclerotic vessel wall [4, 6]. The contact ratio of inflammatory cell content and collagen in the entire plaque remained the same throughout plaque development. This is indicative of development of stable plaques $[1,6]$.

In the largest (class 3) plaques, collagen between vSMCs in the tunica media was labeled, indicating local disruption of IEL [13] as a consequence of plaque progression. In cores of such advanced lesions, co-localization of inflammatory cells and collagen could not be assessed in mounted arteries due to a lack of fluorescent signal. Observations in fresh arterial rings with additional labeling revealed that these cores contain large numbers of inflammatory cells (such as foam cells with many fat droplets) and only little collagen (fig. 3-4) [26]. This implies that especially in larger advanced plaques (which contain larger lesion cores) co-localization between inflammatory cells and collagen in the whole 
plaque may have been overestimated. In contrast to lesion cores, fibrous caps were found to be densely packed with collagen and little inflammatory cells, but co-localization of inflammatory cells and collagen did increase with age. This indicates that fibrous caps provide strength to the plaque while the cores become more and more unstable, suggesting that plaque destabilization is initiated from the core(s) of the plaque and progresses towards the outside borders such as the fibrous cap or the plaque shoulder. Yet, it is important to realize that presence of inflammatory cells alone does not provide any information regarding activation state of such cells and, hence, presence of active enzymes that can break down extracellular matrix components.

In the tunica adventitia, the relative number of inflammatory cells was significantly higher at lesion sites, indicating a local increase in inflammation. Adventitial accumulation of inflammatory cells has been described previously in non-viable murine atherosclerotic aortas and human arteries $[17,27,28]$, and suggests that the inflammatory reaction involved in atherosclerosis does not only occur in the tunica intima but also in the tunica adventitia. Unlike observations by others [17], we did not detect any inflammatory cells in the tunica media. Since we know that anti-CD $11 \mathrm{~b} / \mathrm{PE}$ can penetrate into the tunica media (own observation), lack of inflammatory cells in the tunica media in our study is not caused by a labeling artifact. The increase of inflammatory cells in the tunica adventitia at lesion sites is therefore not caused by migration of cells originating from the intimal lesion, but through another pathway. Vasa vasorum has been suggested to be responsible for the increase of adventitial inflammatory cells with plaque progression in aortas of aged mice [29]. However, in young mice, including the ages used in this study, the relatively thin walled carotid arteries lack vasa vasorum (own observations) [29, 30], suggesting that the increase of inflammatory cells might be caused by a local inflammatory process that attracts inflammatory cells from surrounding tissues.

The two-photon microscope setup used in this study did not allow complete visualization of the largest lesions (fig. 6). Loss of signal deeper in these plaques caused that both fibrous cap(s) and endothelial cell lining bordering the lumen could not be visualized. As a result, we were not able to quantify cell and collagen content in the largest lesion cores in mounted arteries. This loss of signal is most likely due to the lipid rich content of these lesion cores in advanced plaques (fig. 4). Enhancing penetration depth of two-photon laser excitation light by narrowing pulse width, and thus increasing probability of two-photon excitation, may enable imaging of entire intact advanced plaques [31], provided that the cores are actually stained with the fluorochromes. 
In conclusion, this study is the first to visualize and describe the association of collagen and inflammatory cells during plaque development in intact still viable mounted carotid arteries of ApoE $\%$ mice at subcellular level. Visualization of mounted arteries with TPLSM enables further study of various aspects of atherosclerosis in a physiologically relevant environment.

\section{Acknowledgements}

Gregorio Fazzi (Maastricht University, Department of Pharmacology) helped with histology, Monica Breurken (Department of BME, Eindhoven University of Technology) with preparation of CNA35/OG488. TPLSM was obtained via grant No.902-16-276 from the medical section of the Netherlands Organization for Scientific Research (NWO). 
Figures
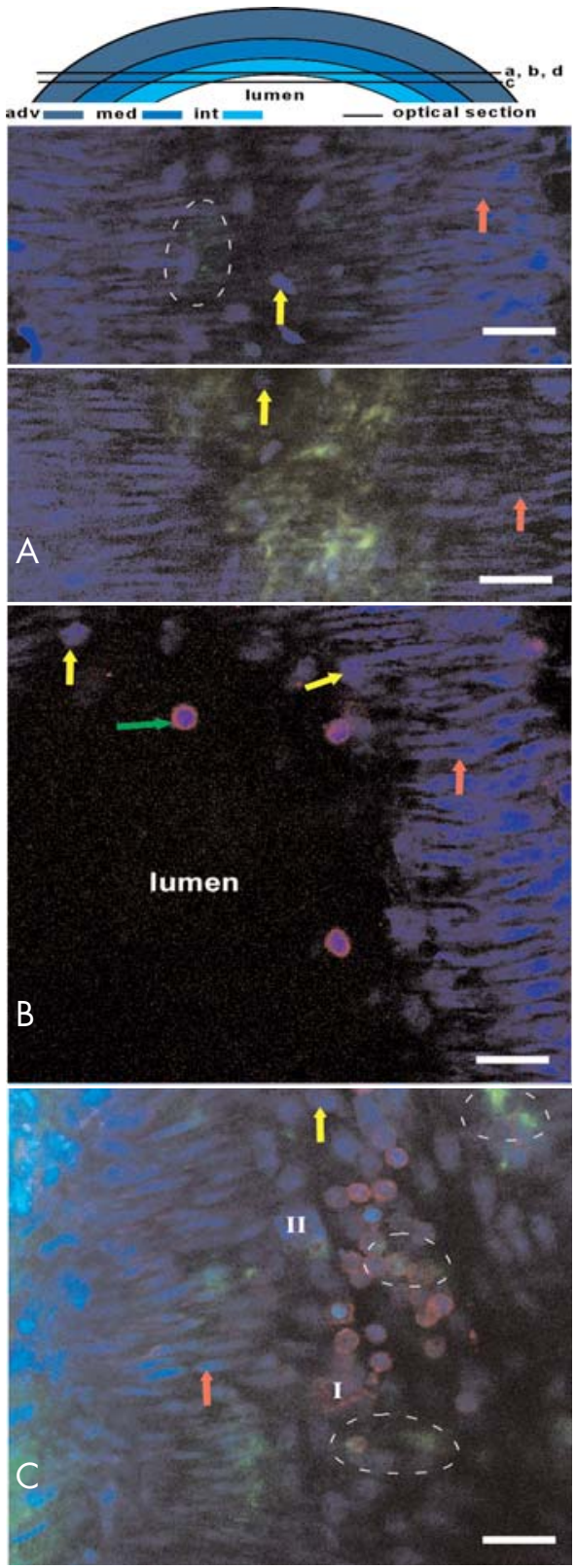

Figure 1

Optical sections of tunica intima in a healthy common carotid segment of C57BL6/J (A) and ApoE $\%$ mouse (B-D) (each 21 weeks). Arteries were stained for cell nuclei (SYTO41; blue), CNA35/OG488 (collagen, green) and antiCD 1 1b/PE (inflammatory cells, red). Section (C) was stained with SYTO41 and anti-CD 1 1b/PE. Bars: $20 \mu \mathrm{m}$. Drawing at top indicates position of displayed sections. (A) Cell nuclei of vSMCs (pink arrow) and endothelial cells (yellow arrow) are clearly visible. Collagen (green) is only sporadically labeled (encircled by white dotted line) and inflammatory cells (red) are absent. At comparable sites in common carotid segments of ApoE $\%$ mice $(B)$, the tunica intima contains a stronger collagen signal (green) between endothelial cells (yellow arrow) and vSMCs (pink arrow). (C) Inflammatory cells (red) were detected adhering to endothelial cells (yellow arrow). (D) Typical example of a class 1 plaque present in carotid bifurcation of ApoE $\%$ mice of both ages. Several inflammatory cells (red) are clearly distinguishable in the distorted tunica intima underneath the endothelial cells (yellow arrow). Furthermore, a CD 11 b/PE-positive foam cell (I) and a CD 1 1b/PE-negative foam cell (II) are visible. Local collagen spots (surrounded by white dotted lines) are noticeable in lesion area. No collagen or inflammatory cells visible between vSMCs (pink arrow) in tunica media. Distribution and orientation of $\mathrm{VSMCs}$ in the tunica media is unaltered. 

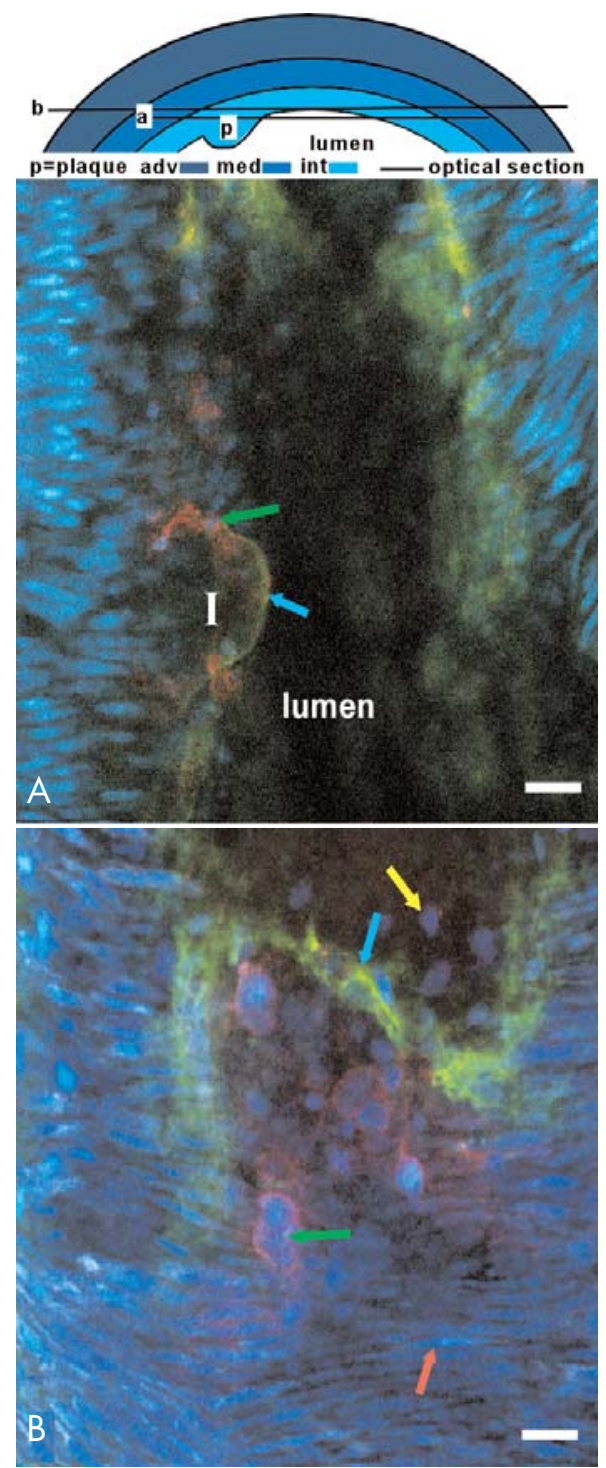

\section{Figure 2}

Optical sections of typical mild plaques (class

2) as observed in tunica intima of ApoE $\%$ mice of $15(A)$ and 21 weeks (B). Arteries were labeled for nuclei (SYTO41; blue), collagen (CNA35/OG488; green), and inflammatory cells (anti-CD $11 \mathrm{~b} / \mathrm{PE}$; red). Drawing at top indicates position of both sections; bars: 20 rm. (A) Class 2 plaque in the carotid bifurcation of a young ApoE $\%$ containing several inflammatory cells and large amounts of collagen. Collagen has fibrous cap-like structure (blue arrow) with a small and weakly fluorescent area (I) inside that contains no collagen and only few inflammatory cells, both typical properties of a small (necrotic) core. In the surrounding arterial wall strongly labeled collagen without a bulb-like structure is observable.

(B) Part of a typical class 2 plaque in carotid bifurcation of $\mathrm{ApoE} \%$ (21 weeks), containing CD 1 1b/PE-positive foam cells (green arrow) and round cells. Collagen is abundant forming a fibrous cap (blue arrow) at the edge of the plaque. Adjacent to the plaque area, endothelial cells are observable (yellow arrow). In the tunica media vSMCs (pink arrow) are homogeneously distributed; their orientation is unaffected. No inflammatory cells or collagen are present in the tunica media. 

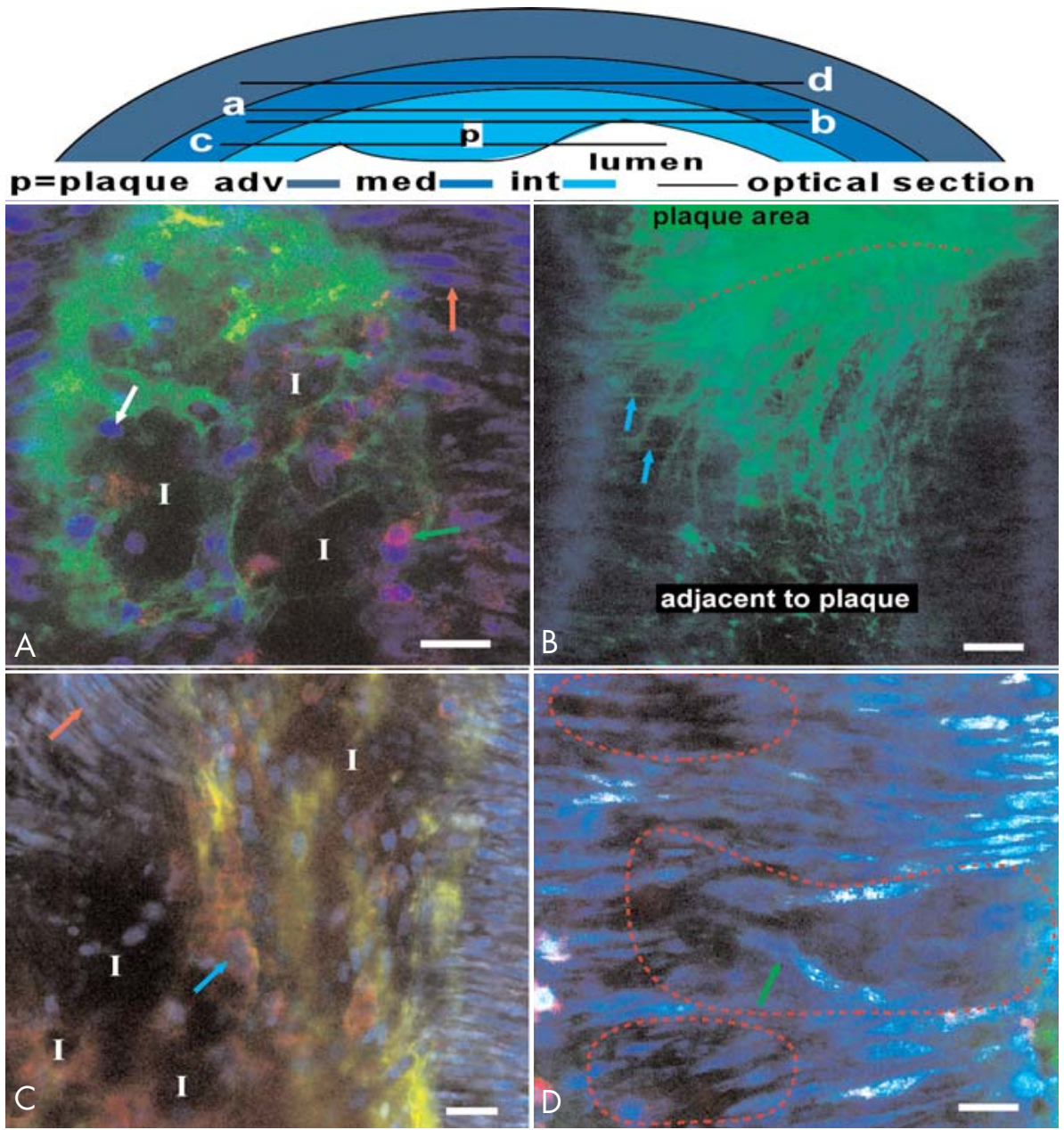

Figure 3

Optical sections of advanced plaques (class 3 ) in tunica intima $(A, B, C)$ and tunica media (D) of carotid bifurcations in ApoE $\%$ mice aged 21 weeks. Arterial segments (A-D) were stained for nuclei (SYTO4 1; blue), collagen (CNA35/OG488; green), and inflammatory cells (anti-CD $11 \mathrm{~b} / \mathrm{PE}$; red).

Segment (B) was stained for collagen only (CNA35/OG488). Bars: $20 \mu \mathrm{m}$; drawing at top indicates position of sections. (A) Advanced plaque with a network-like collagen structure, several lesion cores with weak fluorescent signal (I) and many inflammatory cells (green arrow) and noninflammatory cells (white arrow). (B) Plaque shoulder (red dotted line) containing large amounts of collagen which protrude into area adjacent to the plaque (lower part of the section). Blue is autofluorescence of elastic laminae. Class 3 plaques also appear as an interconnected group of smaller lesions with numerous inflammatory cells, and several lesion cores (C). Plaque borders and lesion cores (I) are less evident; collagen is abundant with less defined appearance. (D) The tunica media flanking advanced plaques contains areas with disrupted vSMCs layer (encircled by red dotted lines) and vSMCs with altered orientation (green arrow). 

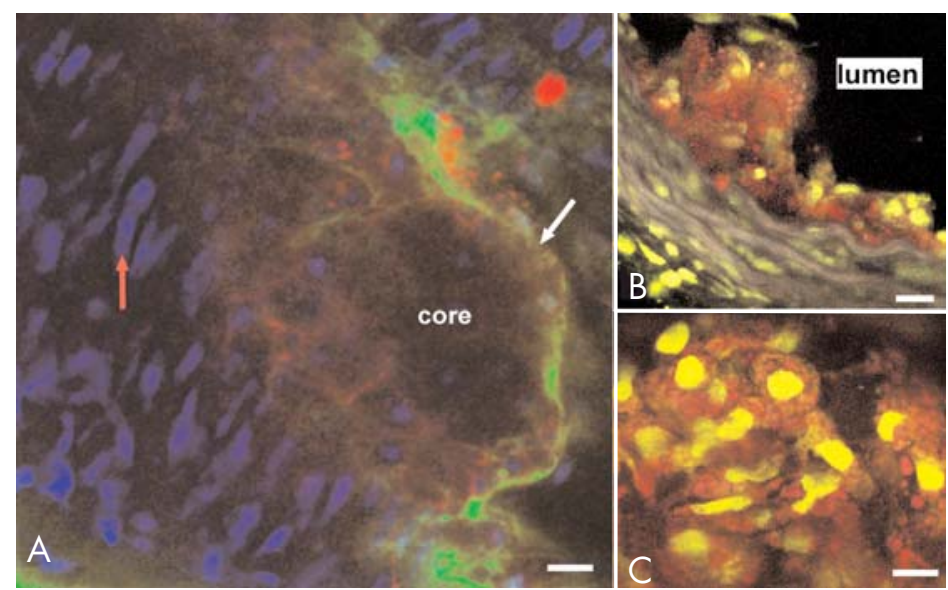

Figure 4

(A) Mounted artery of 21 weeks aged ApoE ${ }^{-}$, containing a class 3 plaque in the bifurcation, labeled for nuclei (SYTO41; blue), collagen (CNA354/OG488; green), and lipids (ORO; red). Bars: 10 $\mu \mathrm{m}$. Note lipid content in core of the lesion, collagen in the fibrous cap (white arrow), and VSMCs in tunica media (red arrow). (B, C) Optical sections of fresh arterial rings stained with ORO and SYTO13 (nuclei, green) further demonstrate that lipids (red) are mainly situated as small droplets inside cells in the lesion cores.
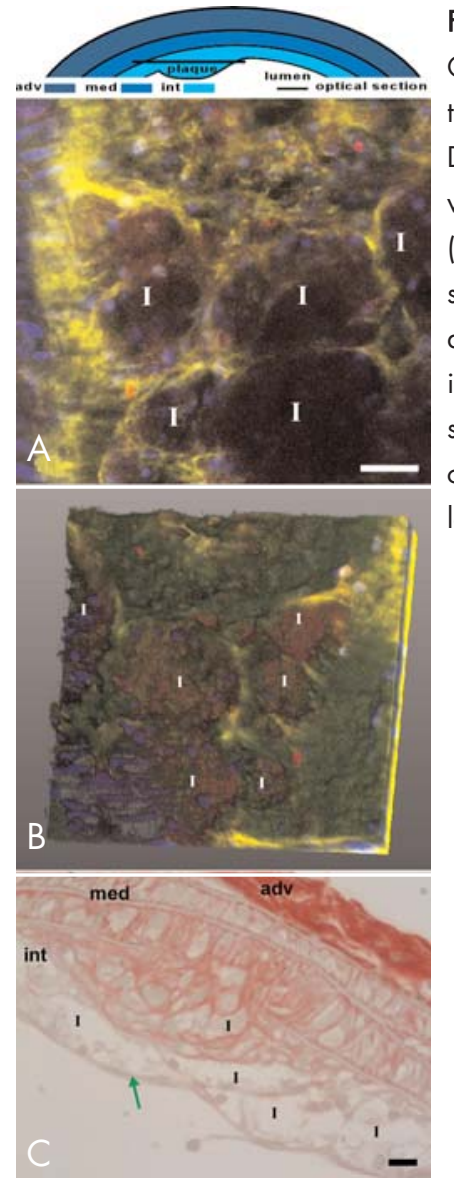

\section{Figure 5}

Optical section (A) and 3D-reconstruction (B) obtained in the tunica intima of class 3 plaque in a 21 weeks aged $\mathrm{ApoE}^{\circ} \%$. Drawing at top indicates position of section; bars: $20 \mu \mathrm{m}$. Artery was stained for collagen (CNA35/OG488; green), nuclei (SYTO41; blue), and lipids (ORO; red). (A) Lesion contains several lipid rich lesion cores (I); collagen has a network-like appearance. (B) The plaque lacks a fibrous cap. Unlike TPLSM images, histological sections of equivalent class 3 plaques (C) stained with picrosirius red (collagen, red) do contain a fibrous cap (green arrow) that consists of collagen. As in TPLSM images, lesion cores (I) are abundant. 


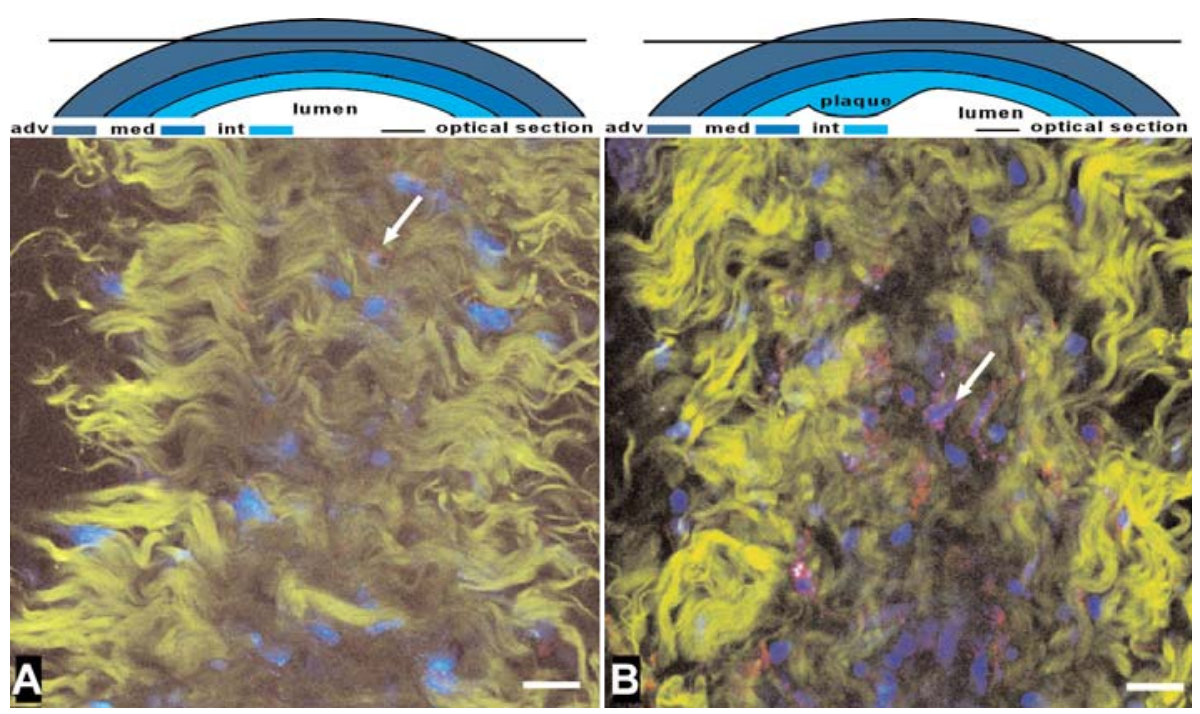

Figure 6

Optical sections in normal tunica adventitia of a C57BL6/J (A) and tunica adventitia (B) of a plaque in an $\mathrm{ApoE}^{-}$, both aged 21 weeks. Drawings on top indicate position of sections, bars: 20 rm. Arteries were stained for collagen (CNA35/OG488, green), nuclei (SYTO41, blue), and inflammatory cells (anti-CD $1 \mathrm{lb} / \mathrm{PE}$, red). In both sections, thin undulating collagen fibers rearrange into thicker wave-like bundles of collagen. In between collagen, nuclei of cells are visible. In the healthy artery (A), only few inflammatory cells (white arrow) are present; in the plaque (B), the number of inflammatory cells (white arrow) is much higher. 


\section{References}

1. Hansson, G.K., Inflammation, atherosclerosis, and coronary artery disease. N Engl J Med, 2005;352(16):1685-95.

2. Libby, P., Changing concepts of atherogenesis. J Intern Med, 2000;247(3):349-58.

3. Lutgens, E., R.J. van Suylen, B.C. Faber, et al., Atherosclerotic plaque rupture: local or systemic process? Arterioscler Thromb Vasc Biol, 2003;23(12):2123-30.

4. Libby, P., P.M. Ridker, and A. Maseri, Inflammation and atherosclerosis. Circulation, 2002;105(9): 1135-43.

5. Katsuda, S. and T. Kaji, Atherosclerosis and extracellular matrix. J Atheroscler Thromb, 2003; 10(5):267-74.

6. Heeneman, S., J.P. Cleutjens, B.C. Faber, et al., The dynamic extracellular matrix: intervention strategies during heart failure and atherosclerosis. J Pathol, 2003;200(4):516-25.

7. Lusis, A.J., Atherosclerosis. Nature, 2000;407(6801):233-41.

8. Lucas, A.D. and D.R. Greaves, Atherosclerosis: role of chemokines and macrophages. Expert Rev Mol Med, 2001;3:1-18.

9. Rodriguez-Feo, J.A., J.P. Sluijter, D.P. de Kleijn, et al., Modulation of collagen turnover in cardiovascular disease. Curr Pharm Des, 2005;11(19):2501-14.

10. Galis, Z.S. and J.J. Khatri, Matrix metalloproteinases in vascular remodeling and atherogenesis: the good, the bad, and the ugly. Circ Res, 2002;90(3):251-62.

11. Denk, W. and K. Svoboda, Photon upmanship: why multiphoton imaging is more than a gimmick. Neuron, 1997;18(3):351-7.

12. Megens, R.T., S. Reitsma, P.H. Schiffers, et al., Two-photon microscopy of vital murine elastic and muscular arteries. Combined structural and functional imaging with subcellular resolution. J Vasc Res, 2007;44(2):87-98.

13. Megens, R.T.A., M.G.A. oude Egbrink, J.P.M. Cleutjens, et al., Imaging collagen in intact and viable healthy and atherosclerotic arteries using fluorescently-labeled CNA35: towards molecular imaging of collagen in atherosclerotic plaques. Molecular Imaging, 2007;6(4):247-60.

14. van Zandvoort, M., W. Engels, K. Douma, et al., Two-photon microscopy for imaging of the (atherosclerotic) vascular wall: a proof of concept study. J Vasc Res, 2004;41(1):54-63.

15. Ferrara, D.E., D. Weiss, P.H. Carnell, et al., Quantitative 3D fluorescence technique for the analysis of en face preparations of arterial walls using quantum dot nanocrystals and twophoton excitation laser scanning microscopy. Am J Physiol Regul Integr Comp Physiol, 2006;290(1):R 1 14-23.

16. Boulesteix, T., A.M. Pena, N. Pages, et al., Micrometer scale Ex Vivo multiphoton imaging of unstained arterial wall structure. Cytometry A, 2006;69(1):20-6.

17. Maffia, P., B.H. Zinselmeyer, A. lalenti, et al., Images in cardiovascular medicine. Multiphoton microscopy for 3-dimensional imaging of lymphocyte recruitment into apolipoprotein-Edeficient mouse carotid artery. Circulation, 2007;1 15(11):e326-8.

18. Krahn, K.N., C.V. Bouten, S. van Tuijl, et al., Fluorescently labeled collagen binding proteins 
allow specific visualization of collagen in tissues and live cell culture. Anal Biochem, 2006;350(2): 177-85.

19. Bernatchez, S.F., M.R. Atkinson, and P.J. Parks, Expression of intercellular adhesion molecule1 on macrophages in vitro as a marker of activation. Biomaterials, 1997;18(20):1371-8.

20. Koopman, R., G. Schaart, and M.K. Hesselink, Optimisation of oil red O staining permits combination with immunofluorescence and automated quantification of lipids. Histochem Cell Biol, 2001;116(1):63-8.

21. Konig, K., Multiphoton microscopy in life sciences. J Microsc, 2000;200 ( P+ 2):83-104.

22. Gijbels, M.J., M. van der Cammen, L.J. van der Laan, et al., Progression and regression of atherosclerosis in APOE3-Leiden transgenic mice: an immunohistochemical study. Atherosclerosis, 1999; 143(1):15-25.

23. Crisby, M., G. Nordin-Fredriksson, P.K. Shah, et al., Pravastatin treatment increases collagen content and decreases lipid content, inflammation, metalloproteinases, and cell death in human carotid plaques: implications for plaque stabilization. Circulation, 2001;103(7):92633.

24. Junqueira, L.C. and J. Carneiro, Basic Histology. Eleventh edition ed. 2005, New York: McGraw-Hill Medical Publishing Division.

25. Simionescu, M., Implications of early structural-functional changes in the endothelium for vascular disease. Arterioscler Thromb Vasc Biol, 2007;27(2):266-74.

26. Stary, H.C., A.B. Chandler, R.E. Dinsmore, et al., A definition of advanced types of atherosclerotic lesions and a histological classification of atherosclerosis. A report from the Committee on Vascular Lesions of the Council on Arteriosclerosis, American Heart Association. Circulation, 1995;92(5):1355-74.

27. Wilcox, J.N. and N.A. Scott, Potential role of the adventitia in arteritis and atherosclerosis. Int J Cardiol, 1996;54 Suppl:S21-35.

28. Moos, M.P., N. John, R. Grabner, et al., The lamina adventitia is the major site of immune cell accumulation in standard chow-fed apolipoprotein E-deficient mice. Arterioscler Thromb Vasc Biol, 2005;25(1 1):2386-91.

29. Langheinrich, A.C., A. Michniewicz, D.G. Sedding, et al., Correlation of vasa vasorum neovascularization and plaque progression in aortas of apolipoprotein $E(-/-) / /$ low-density lipoprotein(-/-) double knockout mice. Arterioscler Thromb Vasc Biol, 2006;26(2):347-52.

30. Hayden, M.R. and S.C. Tyagi, Vasa vasorum in plaque angiogenesis, metabolic syndrome, type 2 diabetes mellitus, and atheroscleropathy: a malignant transformation. Cardiovasc Diabetol, 2004;3(1):1.

31. McConnel, G., Improving the penetration depth in multiphoton excitation laser scanning microscopy. Journal of Biomedical optics, 2006; 11 (5):054020 1-7. 


\section{Chapter 6}

\section{Imaging Enhanced NO levels in Stimulated Sprouting Cultured Endothelial Cells.}

submitted

Marc van Zandvoort ', Remco Megens ', Albert Huisman 2, Wim Engels ', Karin van 't Wout ${ }^{3}$, Herbert de Groot ${ }^{4}$, Reiner Sustmann ${ }^{5}$, Hans-Gert Korth ${ }^{5}$, and Ernst E. van Faassen ${ }^{6}$

'Department of Biomedical Engineering, Cardiovascular Research Institute Maastricht (CARIM), Maastricht University, Maastricht, The Netherlands. 'Department of Clinical Chemistry and Haematology, University Medical Center Utrecht, Utrecht, The Netherlands. ${ }^{3}$ Department of Vascular Medicine, University Medical Center Utrecht, Utrecht, The Netherlands. "Institut für Physiologische Chemie, Universitätsklinikum Essen, Essen, Germany. ${ }^{5}$ Instituł für Organische Chemie, Universität Duisburg-Essen, Essen, Germany. ${ }^{\circ}$ Debye Institute, Section Interface Physics, Utrecht University, Utrecht, The Netherlands. 


\section{Abstract}

We characterized and applied a Fluorescent Nitric-oxide Cheletropic Trap (FNOCT) for sensitive NO detection using two-photon microscopy. Under physiological conditions the membrane-permeable ester FNOCT-5 neither significantly inhibits enzymatic NO production, nor acts as superoxide scavenger. Although FNOCT-5 inhibited eNOS and iNOS in a dose-dependent manner $\left(I C_{50}=86 \mu \mathrm{M}\right.$ vs. $\left.163 \mu \mathrm{M}\right)$, addition of albumin $(10 \mathrm{mg} / \mathrm{ml})$ abolished this effect. We applied FNOCT-5 $(50 \mu M)$ to sprouting and non-sprouting HUVECs on beads in fibrin matrix. Intracellular enzymes transform FNOCT-5 into its accumulating dicarboxylate FNOCT-4. On binding NO, FNOCT-4 fluorescence undergoes a red to blue shift. We used the ratio of blue to red fluorescence $(\mathrm{R})$ as measure of NO production. Sprouting and non-sprouting cells not additionally stimulated did not produce detectable amounts of NO $(R=1.12 \pm 0.12$ vs. $0.95 \pm 0.15)$. However, in the same cell culture, sprouting cells showed significantly higher NO production than their non-sprouting counterparts when stimulated by calcium ionophore $(R=1.56 \pm 0.10$ vs. $R=0.81 \pm 0.12$ ). Stimulus with acetylcholine gave even larger differences $(R=6.1 \pm 2.1$ vs. $R=0.8 \pm 0.2)$. These data show for the first time that microscopy of cheletropic traps allows detection of local variations in NO production with subcellular resolution in physiologically relevant 3D-systems. 


\section{Introduction}

Nitric Oxide ( $\mathrm{NO}$ ) is an important messenger molecule in a variety of physiological actions in the peripheral tissue as well as the central nervous system [1, 2]. The free radical NO is synthesized via the conversion of Larginine to L-citrulline by a family of enzymes called "NO synthases" (NOS) [3]. In tissues, the lifetime of $\mathrm{NO}$ is in the order of seconds. Its zero electric charge allows $\mathrm{NO}$ to diffuse rapidly into adjacent cells. NO does not seem to be essential for vascular development in embryos, but it is directly implicated in pathological angiogenesis, atherogenesis, revascularization of ischemic tissue, and re-endothelialization of damaged vessel walls [4-7]. To study the role of $\mathrm{NO}$ in these disease processes, direct detection of $\mathrm{NO}$ in living cells is a crucial step.

Basal levels of $\mathrm{NO}$ in viable tissues are of the order of 10-100 nM [8], but may rise to micromolar levels under conditions of ischemia, septic shock, or allograft rejection. The nanomolar concentrations usually encountered require sensitive detection of $\mathrm{NO}$ production with spatial resolution on a cellular scale with $\mathrm{NO}$ sensitive probes. A number of fluorescent traps for $\mathrm{NO}$ are available [9] but all but one suffer from toxicity or a lack of specificity for $\mathrm{NO}$ due to the necessity of prior transformation of $\mathrm{NO}$ radical into more reactive nitrogen species and/or unwanted reactions with other reactive species which act as impostors for NO radical [10-17]. FNOCTs (Fluorescent Nitric Oxide Cheletropic Traps) have been shown to be efficient and highly selective fluorescent spin traps for the detection of NO [18]. FNOCTs incorporate $\mathrm{NO}$ without prior transformation to higher nitrogen oxides, and thereby achieve high specificity for $\mathrm{NO}$ in its radical state [19].

A good fluorescent NO probe should satisfy six basic requirements: (1) a significant change in fluorescence characteristics (e.g. emission spectral shift like in the case of FNOCTs) on trapping NO; (2) the probe should not be toxic and enable visualization in living cells; $(3)$ the light used for excitation of the probe should not induce a change in the relevant fluorescent characteristics; (4) high selectivity for $\mathrm{NO}$ only; (5) the probe and its reaction product with NO should be insensitive to reactive oxygen species like superoxide or hydrogen peroxide; (6) the probe should not affect the enzymatic activity of the NO synthases. FNOCTs are known to fulfill the first three requirements. In this first part of this paper, we show that the FNOCT-5 / FNOCT-4 system also satisfies the latter three requirements. Subsequently, we apply it to an established in vitro model for the sprouting stage of early angiogenesis. 
In this model, human umbilical vein endothelial cells (HUVECs) are grown on beads in a fibrin matrix. VEGF is used to induce the process of sprouting and the fibrin matrix provides effective scaffolding for the development of endothelial sprouts [20]. The model used in this study differs from the model used by Meineke and colleagues [18] in that it is a three dimensional model with cells in various (sprouting and non-sprouting) stages. Imaging in such a 3D-model requires a microscopic technique that combines 3D-imaging with penetration depth and cellular resolution. Two-photon laser scanning microscopy (TPLSM) [21-23] is such a technique and allows simultaneous excitation at $800 \mathrm{~nm}$ of FNOCT-4 (red emission) and its NO-reacted product FNOCT-4-NOH (blue emission). Therefore, the ratio of blue to red fluorescence of FNOCT is used to image the cellular production of NO. NO production is stimulated by the addition of acetylcholine or calcium ionophore, well-known assays to challenge maximal NOS-capacity. This study demonstrates the potential of FNOCT in combination with TPLSM to differentially image stimulated $\mathrm{NO}$ production in sprouting and non-sprouting cells.

\section{Methods}

\section{Chemicals}

The diacetoxymethylester FNOCT-5 was synthesized as described previously [24] and stored under argon at $-80^{\circ} \mathrm{C}$ until use. Fluorescence spectroscopy showed that the batch of FNOCT-5 $\left(\lambda_{\text {max emission }}=600 \mathrm{~nm}\right)$ used in this study contained a small contamination $(<1 \%)$ of the blue fluorescent $(\lambda$ max emission $=$ $430 \mathrm{~nm}$ ) precursor ketone from which FNOCT-5 is generated by photolysis.

SYTO13 $\left(\lambda_{\text {max emission }}=515 \mathrm{~nm}\right.$, green) was purchased from Molecular Probes (Leiden, the Netherlands). eNOS and iNOS were from Cayman (Ann Arbor, MI, U.S.A.l3. H-Arginine was obtained from Amersham Pharmacia Biotech (Roosendaal, the Netherlands). Fibrinogen and plasminogen were obtained from Kordia (Leiden, The Netherlands). Endothelial Cell Basal Medium (EBM) and EGM-2 SingleQuots were obtained from Clonetics (Baltimore, U.S.A.). Penicillin and streptomycin were obtained from Life Technologies (Breda, The Netherlands). All other chemicals were from Sigma (St. Louis, MO, U.S.A.). Cell culture materials and RPMI-1640 medium were from Gibco (Paisley, U.K.). The dextrane Cytodex-3 beads were purchased from Amersham Biosciences.

\section{Determination of NO production by NOS: Radiolabeled citrulline assay}

NOS activity was determined by quantifying the conversion of $L-\left[2,3,4,5-{ }^{3} \mathrm{H}\right]$ arginine into $\mathrm{L}-\left[2,3,4,5-{ }^{3} \mathrm{H}\right]$ citrulline, mainly as described previously [25]. Briefly, for the determination of the effect of FNOCT-5 on the enzymatic activity of NOS, $42 \mu \mathrm{g}$ eNOS (or $57 \mathrm{\mu g}$ iNOS) was incubated for 60 minutes at 37 
${ }^{\circ} \mathrm{C}$ in $100 \mu \mathrm{L} 10 \mathrm{mM}$ Tris buffer (pH 7.4) containing (in mmol/L) dithiothreïtol (0.1), $\mathrm{CaCl}_{2}(0.1)$ (or in the case of iNOS Mg-acetate (1.0)), L-arginine (0.5) and NADPH (0.5); (in umol/L) flavin mononucleotide (FMN)(1.0), flavin adenin dinlucleotide $(F A D)(1.0)$, gluthathione (100), tetrahydrobiopterin $\left(\mathrm{BH}_{4}\right)(10)$, calmodulin $(20 \mu \mathrm{g} / \mathrm{mL})$; Bovine Serum Albumin (BSA) $100 \mu \mathrm{g} / \mathrm{mL}$; and L$\left[2,3,4,5-{ }^{3} \mathrm{H}\right]$ arginine $(3.7 \mathrm{KBq})$. For the comparison between the fluorescence assay and the conversion assay, the conditions were chosen similar to the fluorescence assay. Stock solutions of FNOCT-5 were freshly prepared in DMSO and care was taken that the final DMSO concentration in all experiments was equal. All measurements were performed in duplo except were stated otherwise. The NOS activity was calculated from the percentage conversion of $\left[{ }^{3} \mathrm{H}\right]$ arginine into $\left[{ }^{3} \mathrm{H}\right]$ citrulline.

Determination of superoxide trapping rates: EPR spectroscopy using competitive superoxide trapping

The reaction rate between superoxide and FNOCT- 5 was determined at $37^{\circ} \mathrm{C}$ by comparison with the known reaction rate of superoxide with the spin trap DMPO*. In a xanthine oxidase (XO) solution (Phosphate Buffered Saline, PBS buffer, pH 7.4), excess hypoxanthine substrate induces a steady and constant rate of superoxide production. The highly mobile superoxide molecules can react with DMPO to give paramagnetic radical adducts which are detected and quantified by means of EPR spectroscopy. In presence of FNOCT-5, the possible reaction between FNOCT-5 and superoxide should manifest itself as a reduction in the yield of DMPO-OOH radical adducts.

The EPR spectra were recorded at $37{ }^{\circ} \mathrm{C}$ on a modified Bruker ESP300 spectrometer (Bruker, Karlsruhe, Germany) operating near $9.4 \mathrm{GHz}$. The samples consisted of $30 \mu \mathrm{L}$ aqueous solution drawn by syringe into a quartz capillary (1 mm i.d.). The capillary was placed inside a quartz tube (length 15 $\mathrm{cm}, 3 \mathrm{~mm}$ i.d.) and located in the center of a Bruker STI resonator (operating in the TE 102 mode with an unloaded $Q=3800$ ). The sample temperature was kept at $37 \pm 1^{\circ} \mathrm{C}$ by a nitrogen gas flow from a Bruker ER4111VT temperature controller.

The experiments were performed in PBS buffer $\left(\mathrm{pH} 7.4,37^{\circ} \mathrm{C}\right)$ containing 100 $\mathrm{mM}$ DMPO in a total volume of $100 \mu \mathrm{L}$. Superoxide radicals were generated

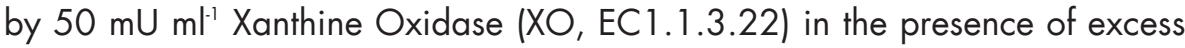
hypoxanthine $(\mathrm{HX})(0.5 \mathrm{mM})$. The formation of DMPO-OOH adducts could be suppressed by addition of superoxide dismutase (SOD).

\section{Cell culture on beads}

HUVECs were freshly isolated from human umbilical cord veins as described 
previously [26]. The cells were cultured in RPMI-1640 medium containing 20 $\%(\mathrm{v} / \mathrm{v})$ heat-inactivated human serum and $200 \mathrm{\mu g} \mathrm{ml}^{-1}$ penicillin and streptomycin. Endothelial cells of the second passage or third passage were used in the bead assay. It involved two stages of cell growth followed by a final sprouting stage. In the first growth stage, the cells were grown at $37^{\circ} \mathrm{C}$ to a confluent monolayer on the bottom of a $75 \mathrm{~cm}^{2}$ culture-flasks under a controlled atmosphere of $20 \%$ oxygen, $5 \%$ carbon dioxide, and $75 \%$ nitrogen. In this first stage, confluence was obtained after 5-7 days. The confluent cultures were washed with PBS buffer and trypsinized. For the second growth stage, the cells were transferred to a flask containing dextrane microcarrier beads in endothelial basal medium EBM. The medium was supplemented with EGM-2 SingleQuots, which contains growth factors and cytokines to promote growth and proliferation of the endothelial cells. The dextrane beads had an average diameter of ca $200 \mu \mathrm{m}$ and were coated with porcine gelatin for good adherence of the endothelial cells. The HUVECs were cultured to confluence on the beads under continuous but slow stirring at $37{ }^{\circ} \mathrm{C}$ under a controlled atmosphere of $20 \%$ oxygen, $5 \%$ carbon dioxide, and $75 \%$ nitrogen. In this second growth stage, confluence was obtained after 2-3 days. For the final sprouting stage the suspended beads were removed from the growth flask by pipette and embedded in a transparent fibrin matrix that allows diffusive transport of nutrients, oxygen and metabolites while immobilizing the beads for microscopic investigation. This fibrin matrix was prepared by adding $0.1 \mathrm{U} \mathrm{ml}^{-1}$ thrombin to a mixture of $2 \mathrm{mg}(\mathrm{mll})^{-1}$ fibrinogen, $2 \mathrm{mg} \mathrm{ml}^{-1}$ sodium citrate, $14 \mathrm{mM}$ $\mathrm{NaCl}, 3 \mu \mathrm{g} \mathrm{ml} \mathrm{l}^{-1}$ plasminogen in RPMl-1640 medium. This low-viscosity mixture was poured into 6-wells plates, the beads being mixed in immediately after. The mixture became gelatinic by polymerization on a timescale of several minutes. Before the induction of sprouting, the thrombin in the medium was inactivated by washing with RPMI-1640 containing $10 \%$ human serum and 10 $\%$ Fetal Calf Serum (FCS) for 2 hours. As a final step, the liquids in the fibrin matrix were replaced with EBM medium supplemented with EGM-2 SingleQuots to promote sprouting of the endothelial cells. This medium was free of phenol-red to avoid interference with fluorescence microscopy, and was refreshed every two days. The sprouting stage developed at $37{ }^{\circ} \mathrm{C}$ under a controlled atmosphere of $20 \%$ oxygen, $5 \%$ carbon dioxide, and $75 \%$ nitrogen. Prominent growth of capillary sprouts into the fibrin matrix could be observed microscopically after 4 days.

\section{NO detection.}

For NO detection, the sprouting samples were labeled with the cheletropic trap FNOCT-5 [18]. FNOCT-5 is an uncharged acetoxymethylester and readily 
penetrates cell membranes. The trap accumulates in cells because the cellular esterases transform the acetoxymethylester into the dicarboxylate of the dicarboxylic acid FNOCT-derivative FNOCT-4, which due to its negative charges cannot leave the cell [18]. FNOCT-4 is the actual intracellular NO trap and shows weak red fluorescence $\left(\lambda_{\text {max emission }}=600 \mathrm{~nm}\right)$.<smiles>CC(=O)COC(=O)COC(=O)COC(C)=O</smiles>

FNOCT-5<smiles></smiles>

FNOCT-4<smiles></smiles>

FNOCT-4NOH

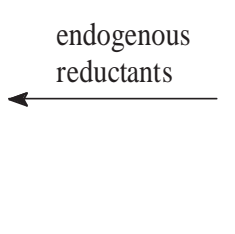<smiles>CC(C)[N+](=O)[O-]</smiles>

FNOCT-4NO

When FNOCT-4 binds to NO radicals, a new nitroxide radical is produced (FNOCT-4NO). This intermediate is not fluorescent and in biological systems rapidly hydroxylates to the strongly blue fluorescent FNOCT-4NOH $\left(\lambda_{\max }\right.$ emission $=460 \mathrm{~nm}$, with a tenfold increase in fluorescence intensity [18]) It should be noted that FNOCT-5 and FNOCT-5NOH show identical fluorescence characteristics as their counterparts FNOCT-4 and FNOCT-4NOH, respectively [24].

FNOCT-5 was dissolved to a $5 \mathrm{mM}$ stock solution in DMSO supplemented with $10 \%$ Pluronic F127 to improve solubility. The fibrin gel was loaded with a solution of $50 \mu \mathrm{M}$ FNOCT-5 in PBS. Two-photon microscopy confirmed that FNOCT-5 slowly diffused through the gel matrix. The diffusion speed of FNOCT in the fibrin gel was determined to be $200 \pm 20 \mu \mathrm{m}$ per hour at $37^{\circ} \mathrm{C}$. After reaching an endothelial cell, the FNOCT-4 dianions were accumulated within 30 minutes. Where visualization of cell nucleus was desired, SYTO13 was added to the samples to reach a final concentration of approximately $0.5 \mu \mathrm{M}$. At these low concentrations SYTO13 only labels the cell nucleus.

During microscopy, the gel samples were exposed to ambient temperature and 
atmosphere. HUVECs were stimulated to generate nitric oxide by addition of either acetylcholine (50 $\mu$ M, Sigma, St. Louis, MO, USA) or calcium ionophore

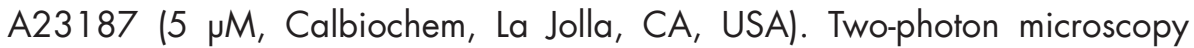
showed that calcium ionophore reached and stimulated the first cells within half an hour. Acetylcholine diffused through the fibrin gel at a much slower rate and only after 6 hours the first cells were reached and stimulation was observed.

\section{Microscopy set-up}

A standard Biorad Multiphoton System Radiance 2100 (Biorad, Hemel Hempstead, United Kingdom) was used in TPLSM mode. The excitation source was a Spectra-Physics Tsunami Ti:Saphire laser (Spectra-Physics, Mountain view, CA, USA). The laser is tuned and mode-locked using a prism sequence and a slit. The position of the slit tunes the output wavelength, while the width of the slit changes the temporal width of the pulses. For most experiments the laser was tuned and mode-locked at $800 \mathrm{~nm}$, producing light pulses with duration of about $140 \mathrm{fs}$ (repetition rate $82 \mathrm{MHz}$ ). The pulses reached the sample through the microscope objective $160 \mathrm{X}$, water dipping, numerical aperture 1.0, working distance $2 \mathrm{~mm}$ ), connected to an upright Nikon E600FN microscope (Nikon Corporation, Tokyo, Japan). An optical zoom in the scan head achieved further magnification. When desirable the fluorescence is detected by three independent photomultipliers for the blue, green and red wavelength regions (430-470 nm; 508-523 nm and above $570 \mathrm{~nm}$ respectively). For visualization, the so-obtained three separate images (coded blue, green and red, respectively), were combined into a single image. However, the intensities in the three separate channels remain available and are used for quantification purposes (see microscopy data analysis). The excitation wavelength of $800 \mathrm{~nm}$ was chosen since we found that at this wavelength both traps and adducts were effectively excited and background cellular autofluorescence is minimalized and undetectable. No additional image processing was performed. For imaging of the endothelial cells on the beads, an imaging speed of $0.1 \mathrm{~Hz}$ was used to improve signal-to-noise ratio. Images were analyzed using acquisition software (Laser sharp 3.0, Biorad, Hemel Hempstead, United Kingdom), and Image-Pro Plus 6.0 (quantitative image analysis software, Media Cybernetics Inc, Silver Spring, USA). 3Dreconstructions were created with the 3D-reconstructor 5.1 software package for Image-Pro Plus, from z-stack series (interplane distances of $0.6 \mathrm{\mu m}$; approximate slice thickness $1.5 \mu \mathrm{m}$ ) using Maximum Intensity Projection.

\section{Microscopic data analysis.}

From a z-stack of cells on beads (e.g., from figures 3 and 4) the ratio of blue 
and red fluorescence (R) of a single cell was determined by determining the ratio of blue to red fluorescence for 5 independent line profiles (using ImagePro Plus 6.0). Care was taken to avoid the crossing of selected lines with nuclear regions that did not take up the probe. The values of $R(R \pm S E)$ indicated in the text are then the averages of a number $(\mathrm{m})$ of single cell values in a number $(n)$ of independent experiments (no intervention, sprouting: $n=2$, $m=17$; no intervention, non-sprouting: $n=2, m=10$; acetylcholine, sprouting: $n=3, m=35$; acetylcholine, non-sprouting: $n=3, m=10$; calcium ionophore, sprouting: $n=3, m=20$; calcium ionophore, non-sprouting: $n=3, m=10$ ). Sprouting and non-sprouting cells were discriminated on the basis of the presence of cellular extrusions in 3D-stacks of beads. All relevant settings for intensities (laser power, filter choice, gain, offset) were equal, so that values of $\mathrm{R}$ for the used images can be compared. Since cellular autofluorescence was virtually absent (less then $1 \%$ of the lowest red FNOCT intensity in unstimulated cells), no autofluorescence correction was needed for the R value. However, the value of $R$ cannot directly be related to concentrations, since that would require tedious calibration procedures for the specific settings used. This was beyond the scope of this study. Differences in ratios were tested for significance using one way ANOVA with a Bonferroni correction. The first test involved the groups of calcium ionophore intervention and no intervention. The second test compared the acetylcholine intervention with the no intervention. A value of $\mathrm{p} \leq 0.05$ was considered to be statistically significant. The SPSS 13.0 software package (SPSS Inc., Chicago, USA) was used for statistical analysis.

\section{Results}

FNOCT-5 does not affect enzymatic activity of eNOS and iNOS under physiological conditions

The influence of FNOCT-5 ester on the enzymatic activity of eNOS and iNOS was established by the ${ }^{3} \mathrm{H}$-Arginine $\longrightarrow{ }^{3} \mathrm{H}$-Citrulline conversion assay. In absence of FNOCT-5, the NO production of eNOS and iNOS was found as

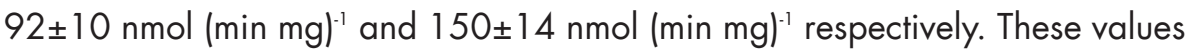
are in reasonable agreement with production rates published previously [27, 28]. The half maximum inhibiting concentration $\left(I C_{50}\right)$ is determined by dose dependent inhibition of the conversion by FNOCT-5. FNOCT-5 inhibited both eNOS and iNOS in a dose dependent manner with an $\mathrm{IC}_{50}$ of $86 \pm 14 \mathrm{M}$ and $163 \pm 63 \mathrm{M}$ respectively (fig. 1). These $I_{50}$ values are higher than the volumeaveraged [FNOCT-5] concentration $(\approx 50 \mu \mathrm{M})$ used in applications, but it should be kept in mind that the intracellular FNOCT-5 concentrations are usually higher due to the intracellular accumulation process. The inhibition capacity of FNOCT-5 is related to its modest solubility in water, since its 
hydrophobic character gives the FNOCT-5 a preference for the cellular lipid and protein compartments [24]. Therefore, in the absence of other low-polarity compartments in the buffer solution, the solubility characteristics will result in artificially high concentrations of FNOCT-5 ester on NOS proteins, thus overestimating the effect of FNOCT-5 on enzymatic activity of iNOS and eNOS.

We therefore repeated the inhibition experiment in the presence of physiological quantities of bovine serum albumin (BSA). An excess FNOCT-5 concentration of $320 \mu \mathrm{M}$ is strongly inhibiting both eNOS and $\mathrm{N} N \mathrm{~S}$ in the absence of BSA. However, the presence of $10 \mathrm{mg} \mathrm{m}^{-1}$ BSA restores eNOS and iNOS activity to $91 \%$ and $82 \%$, respectively, of maximum value.

\section{FNOCT-5 does not scavenge superoxide}

The scavenging capacity for the reaction of FNOCT-5 with superoxide $(\mathrm{pH} 7.4$, $37^{\circ} \mathrm{C}$ ) was investigated by competitive spin trapping with DMPO using $\mathrm{XO} / \mathrm{HX}$ as a superoxide producing system. By EPR, we observed that the presence of FNOCT-5 in concentrations up to $1.25 \mathrm{mM}$ did not affect the formation of DMPO adducts. At the same time, exposure of $50 \mu M$ FNOCT-5 to superoxide radicals did not reduce the intensity or spectral shape of its red fluorescence. Apparently, the presence of FNOCT-5 does not inhibit the enzymatic activity of Xanthine Oxidase. Also, FNOCT-5 does not react significantly with superoxide even when used at high concentrations. The limited sensitivity of EPR implies that we can only determine an upper limit for the reaction rate of FNOCT-5 with superoxide: $k<10^{2}(\mathrm{Ms})^{-1}$. The true value may well be far smaller than this upper limit, or even essentially zero. This should be compared with the value for the reaction rate of FNOCT-5 in buffer with NO (160 $\left.\mathrm{M}^{-1} \mathrm{~s}^{-1}\right)$ as found before [29].

Similarly, in vitro steady-state fluorescence spectroscopy showed that the bluefluorescent FNOCT-NOH adducts do not react with superoxide radicals from the addition of excess potassium superoxide. Similarly, the fluorescence of FNOCT-4-NOH was stable against addition of excess hydrogen peroxide (data not shown).

\section{Sprouting endothelial cells have higher NO production after stimulation}

The production of $\mathrm{NO}$ by endothelial cells growing on beads was investigated using FNOCT-5 in combination with two-photon fluorescence microscopy. HUVECs in this system grow from the beads into the fibrin matrix in characteristic capillary structures called 'sprouts'. Scanning of the z-stacks and the 3D-reconstructions obtained by TPLSM allows easy discrimination of sprouting and non-sprouting cells. Figure 2 represents a 3D-reconstruction of a 
bead coated with many cells. The cells were exposed to FNOCT-5 for several hours. The probe is internalized and converted to FNOCT-4. No fluorescence above noise level was detected outside the cells, indicating that FNOCT-5 is completely taken up by the cells, where it is converted into FNOCT-4. Both longer sprout networks (S1) as cells with individual sprouts (S2) are visible due to the labeling with FNOCT-4. The cell nucleus (CN) does not contain FNOCT4 , as deduced from the absence of signal above noise level. Figure 3 a shows a typical image of 1 non-sprouting cell and various sprouting cells on the same bead, imaged five hours (in view of the slow diffusion speed of FNOCT-5 through the gel, see materials and methods) after loading with FNOCT-4 traps. Absolute blue and red intensities in sprouting and non-sprouting cells were similar, demonstrating that both types of cell have comparable capacity to accumulate and convert the FNOCT-5 ester to the FNOCT-4 acid form, which represents the actual intracellular NO trap. Five hours after the initiation of cellular loading both blue and red fluorescence intensity remained constant, indicating that at that time accumulation of the probe has saturated. Apparently, basal NO production of both sprouting and non-sprouting cells is below detection level, nor are cells exposed to any other stimulus for $\mathrm{NO}$ production. Finally, this observation also shows that the laser illumination at our wavelength and power level does not cause artefactual blue emission due to photo degradation of the probe molecules. The cells were not further stimulated to produce NO. Cells show a strong red fluorescence and a slightly weaker blue fluorescence, resulting in an overall purple color. The ratio of blue to red fluorescence ( $R$, see materials and methods for procedure) was found to be $1.12 \pm 0.12$ for sprouting and $0.95 \pm 0.15$ for non-sprouting cells. According to the statistical testing these values are not significantly different ( $>>0.05)$. We note that the cells show some residual blue fluorescence due to the aforementioned contamination with the synthetic precursor (see materials and methods) of FNOCT-5. This contamination results in values of $R \approx 1$ and attests to the high but not perfect efficiency of the photolysis used to convert the parent ketone to FNOCT-5. It limits the sensitivity of the FNOCT traps, as the blue fluorescence from true $\mathrm{NOH}$-adducts must approach that of the ketone contamination to be detectable.

Since basal NO production was below our detection limit in both sprouting and non-sprouting cells, we stimulated NO production of the cells with calcium ionophore or acetylcholine, two established actors on eNOS through changes in cellular calcium concentrations. As a typical example fig. $3 \mathrm{~b}$ shows the result for the same cells as in figure 3a, but now half an hour after the addition of calcium ionophore to the medium surrounding the gel. Again, no fluorescence is detectable outside the cells. Non-sprouting cell have maintained their mainly 
purple overall color $(R=0.81 \pm 0.12$, not significantly different from the value in the non-sprouting, unstimulated cells, $p>0.05)$. In contrast, sprouting cells have turned bluer, due to a slight decrease of red fluorescence and a significant increase in blue fluorescence. Although this change is optically only subtle, it is clearly reflected in a significant increase in value of $R$ to $1.56 \pm 0.10(p<0.01$ when compared to both sprouting, no intervention and non-sprouting, with calcium ionophore). This value distinguished the stimulated sprouting cells from their non-sprouting brethren.

Then, cells on beads (different samples) were imaged both before and after stimulation with acetylcholine $(50 \mu \mathrm{M})$. The incubation time was chosen much longer for acetylcholine as this stimulant diffuses through the gel at much slower speed than calcium ionophore (see materials and methods). Additionally, SYTO13 ( $\lambda_{\text {max emission }}=515 \mathrm{~nm}$, green, probe attaches firmly to DNA [30]) was added at low concentration to image the cell nucleus. This did not interfere with the calculation of R, since SYTO13 at such low concentrations was only present in the cell nucleus and only in the green channel. As before with calcium ionophore, two-photon microscopy reveals that no fluorescence is detected outside the cells. Non-sprouting cells have maintained their overall purple color, $\mathrm{R}=0.8 \pm 0.2$, while in sprouting cells a stronger blue fluorescence from the $\mathrm{NO}$ adduct FNOCT-4-NOH is observed and the original red fluorescence of FNOCT-4 has diminished. This is expressed in a value of $R$ that has increased to $6.1 \pm 2.1$ ( $p<0.01$ when compared to both sprouting cells, no intervention and to non-sprouting cells, with acetylcholine). The large error is caused by an apparent dependence of the value of $\mathrm{R}$ on time after stimulus and individual cells. Figure 4 shows a typical 3D-reconstruction of an individual sprouting cells (zoom factor 3), clearly showing the homogeneous blue fluorescence of the cytoplasm, including the sprouts, of this cell (for this specific cell $R=4.0 \pm 0.1)$. In a separate experiment, the transition to blue fluorescence could be abolished $(R=0.9 \pm 0.2)$ by preincubation with $500 \mu \mathrm{M}$ of the NOS inhibitor N-nitro-L-arginine. This observation supports the proposition that the change in fluorescence emission is due to trapping of NO from NOS activity only.

\section{Discussion}

\section{The properties of FNOCT-5}

In experiments with isolated enzyme, FNOCT-5 inhibits both eNOS and iNOS activity with an $\mathrm{IC}_{50}$ of $86 \mu \mathrm{M}$ and $163 \mu \mathrm{M}$, respectively. These values are comparable to the FNOCT concentrations loptimal concentration for the labeling solution was $50 \mu \mathrm{M}$, according to [18],[24] ) used in experiments. 
Therefore, this result seems to imply that FNOCT-5 should be unsuitable to detect $\mathrm{NO}$ in biological systems, especially in view of the fact that the actual intracellular concentration of FNOCT-4 will be higher due to the esterase activity of living cells. However, these in vitro inhibition experiments should be interpreted with care for 2 reasons: (1) given that FNOCT-5 ester has a far higher solubility in low-polarity lipids and protein compartments than in aqueous buffers, in the in vitro experiments FNOCT-5 will preferentially concentrate on NOS proteins in the absence of other low-polarity compartments. This will consequently result in an overestimation of the effect of FNOCT-5 on NOS activity. Indeed, the addition of a small amount of protein (in our case $10 \mathrm{mg} \mathrm{m}^{-1} \mathrm{BSA}$ ) almost completely restores the $\mathrm{NO}$ producing capacity of the enzyme, demonstrating that FNOCT-5 does not act as a specific inhibitor of NOS isoforms. In real biological systems, the protein concentration is generally high (e.g., $75 \mathrm{mg} / \mathrm{ml}$ in human plasma [31], and $200 \mathrm{mg} / \mathrm{ml}$ intracellularly [32]). Therefore, the high protein and lipid content of living cells and tissues provide NOS with good protection against FNOCT inhibition, even at the high intracellular concentrations that can be reached. (2) FNOCT-4 is present intracellularly at much higher concentrations, but is an acid that preferentially localizes in the aqueous phase, away from NOS. Therefore, the effect observed for FNOCT-5 in vitro will apply to a lesser extent for FNOCT-4 intracellularly.

Our EPR experiments have shown that the FNOCT traps do not react significantly with superoxide radicals, and do not inhibit Xanthine oxidase (XO). This finding is significant since Galley et al. have shown [33] that the normal physiology of NOS may be affected by the XO activity in cell cultures. $\mathrm{XO}$ is involved in the metabolism of uric acid and produces large quantities of superoxide when up regulated as a result of ischemia-reperfusion injury or inflammation as occurs in transplant rejection or stimulation with lipopoly saccharide [34-36]. Oxidative stress in general and superoxide radicals in particular are important mediators in $\mathrm{NO}$ metabolism and this may have strong implications in pathophysiology [37]. NO rapidly reacts with superoxide with a rate constant of circa 6.7 * 109-1.9 * $10^{10}(\mathrm{Ms})^{-1}$ [38-40] to form the potent oxidant peroxynitrite [41, 42]. We have shown previously [43] that peroxynitrite may react with FNOCT-5 but that the reaction product is nonfluorescent. As such, peroxynitrite cannot act as an impostor for true $\mathrm{NO}$ in our assay. However, it indicates that what we measure is not the total NO production, but the produced NO that actually survives and is available for direct actions. The present work has shown that FNOCT-5 does not significantly interfere with the enzymatic activity of NOS and xanthine oxidase. 


\section{NO detection in sprouting cells}

Cheletropic traps have been proven to be non-toxic for cultured endothelial cells $[18,24]$. Their main advantage over other fluorescent NO traps is their specificity for $\mathrm{NO}$, since the nitric oxide radical is the only known pathway leading to the blue-fluorescent adduct. One might argue that the formation of this FNOCT4- $\mathrm{NOH}$ is via the hydroxylation of an intermediate FNOCT-4NO and thus its intensity also depends on intracellular reduction of unknown pathways. Indeed, the intermediate product FNOCT-4NO is non-fluorescent and remains unobserved with fluorescence microscopy. However, previous EPR experiments in cell cultures have shown that this nitroxide population remains below detection limit as the hydroxylation of the intermediate to FNOCT-4NOH is facile in biological systems [18]

We applied FNOCT-5 to an in vitro model for the initial sprouting stage of angiogenesis. The endothelium is critical for angiogenesis to occur. Several reports in the literature implicate that endothelium-derived NO plays a role in the pro-angiogenic endothelial capillary formation. Clinical and experimental observations have identified several pathological conditions where deficient angiogenesis is directly linked to deficiency in the NO synthase pathway. Widespread examples are diabetes [44], hyperhomocystenemia [45], or hypercholesterolemia $[46,47]$. The link between angiogenesis and $N O$ is reinforced by observations that suppletion of L-arginine promotes angiogenesis, while inhibition of NO synthase delays the angiogenic process $[48,49]$. Therefore, it was proposed that $\mathrm{NO}$ itself could be a direct modulator of angiogenesis. We restricted ourselves to a model for the sprouting stage of incipient angiogenesis, using two-photon fluorescence microscopy in combination with FNOCT-5/FNOCT-4 to detect the intracellular synthesis of NO. The kinetics of the conversion from red to blue fluorescence, i.e. the rate of NO production, was not yet studied. However, the microscopic resolution allowed morphological identification of sprouting cells, while simultaneously discriminating $\mathrm{NO}$ production in sprouting and non-sprouting cells. Under basal, non-stimulated, conditions the NO production remains below the level of detection for sprouting as well as non-sprouting cells. This is not surprising since $\mathrm{NO}$ is not essential for the sprouting process. To test whether NO production capacity is different in sprouting when compared to non-sprouting cells, we applied a NOS agonist to stimulate the synthesis of NO by the cultured cells. After acetylcholine stimulation, blue fluorescent FNOCT-NOH adducts are formed. Inhibition of this adduct formation by $\mathrm{N}$-nitro-L-arginine shows that NOS is the source of the NO detected.

Previous studies on FNOCTs [24] have shown that in vitro in a closed vessel a tenfold excess of FNOCT leads to a complete trapping of produced NO. This 
poses the question as to the influence of FNOCTs on the physiological role of $\mathrm{NO}$ in cells, since it suggests that $\mathrm{NO}$ produced by cells up to $5 \mu \mathrm{M}$ is completely scavenged. In cell cultures, however, many competing NOconsuming reactions are present. Since the FNOCT-NO reaction is rather slow, in such a system physiological NO sinks, e.g. reactions with heme compounds, are much faster and compete strongly. Thus, only a fraction of produced $\mathrm{NO}$ will be trapped by FNOCT, not influencing physiological function of $\mathrm{NO}$. This has been discussed previously [24].

The three-dimensional spatial resolution afforded by scanning two-photon fluorescence microscopy was used to distinguish stimulated sprouting cells from non-sprouting cells in the same culture. The former have significantly higher levels of NO adducts than non-sprouting cells, even if both cell types exist as close neighbors in the same cell culture and even on the same bead. Our experiments show that sprouting endothelial cells have a higher capacity to produce $\mathrm{NO}$ then their non-sprouting counterparts in the same cell culture. There exists an intricate relationship between $\mathrm{NO}$ and angiogenic growth factors [5]. On the one hand, NO itself was recognized as one of the agents to stimulate angiogenesis in vitro $[7,50,51]$, on the other hand VEGF-derived angiogenesis shows upregulation of endothelial NOS (eNOS) [52] and enhanced NO production [4]. Inhibitors of NO synthase inhibit the angiogenic property of VEGF $[53,54]$ and bFGF [50] in vitro. These findings have been confirmed in a rabbit cornea model for angiogenesis [55] and by impaired angiogenesis [56] in eNOS knockout mice. Additionally, angiogenic effect of statins $[4,57]$ has been attributed to eNOS activation. The above body of data suggests that, although $\mathrm{NO}$ is not crucial for angiogenesis, it reinforces angiogenesis by a positive feedback loop [58]. The results of this study are in line with this suggestion.

This study furthermore shows that highly specific and localized imaging of $\mathrm{NO}$ production in cells in vitro is feasible, although quantification of intracellular $\mathrm{NO}$ concentration was not performed. This would require tedious, preferably cellular, calibration procedures and goes beyond the scope of this study. It confirms the capacity of FNOCT to detect $\mathrm{NO}$ production with cellular resolution, demonstrated before using traditional, 2D-fluorescence microscopy in LPS-stimulated rat alveolar macrophages [29]. Apparently, the $\mathrm{NO}$ is trapped before it can leave the cell by diffusion. This point deserves further consideration. In our cultures, the average FNOCT concentrations used for labeling were $50 \mu \mathrm{M}$, but the action of the cellular esterases accumulate the intracellular FNOCT-4 concentration to an estimated $20 \mathrm{mM}$. The reaction rate of $160 \mathrm{M}^{-1} \mathrm{~s}^{-1}$ with free $\mathrm{NO}$ radicals gives an upper limit of the $\mathrm{NO}$ lifetime of around $0.3 \mathrm{~s}$, but due to additional decay channels besides FNOCT, the actual 
lifetime of $\mathrm{NO}$ will certainly be significantly shorter. From the known diffusion rate for $\mathrm{NO}$ in cells of $3300 \mathrm{\mu m}^{2} \mathrm{~s}^{-1}$ [59] one can calculate the upper limit in diffusion distance to be $30 \mu \mathrm{m}$. Such a value thus indeed strongly supports our assertion that the presented method allows the detection of intracellular NO production of individual cells. This opens avenues for imaging the regulatory pathways involved in the complex interplay between $\mathrm{NO}$ and angiogenesis or atherosclerosis (e.g. effect of oxLDL on $\mathrm{NO}$ production at lesion prone sites [60]). An intriguing question is whether imaging of $\mathrm{NO}$ in more complex, intact tissues (e.g. mouse arteries, aortic rings, heart tissue, kidneys) will be possible using this probe in combination with TPLSM. This is subject of ongoing study.

\section{Acknowledgements}

We thank Cindy Loomans (Department of Vascular Medicine, University Medical Center Utrecht, the Netherlands) for assisting with the cell cultures, $d r$. Peter Boer for the determination of NOS activity, and prof. dr. Anton Jan van Zonneveld for bringing to our attention the bead assay for HUVEC cultures. Dr. Verena Stenert is thanked for valuable synthetic work. The TPLSM was financed by the Dutch Scientific Organization (NWO 902-16-276). Further support was obtained from grant BSIK 03033.

\section{* Footnote}

There is considerable uncertainty in the literature with regard to the absolute value of the rate constant for superoxide trapping by DMPO. Certainly, the protonated form, $\mathrm{HO}_{2}$., is trapped by DMPO with a far higher rate than superoxide itself, so that $\mathrm{pH}$ is a very important parameter. At $\mathrm{pH} 7.4$ we consider the DMPO trapping rate of $26(\mathrm{Ms})^{-1}$ at $25^{\circ} \mathrm{C}$ to be the most reliable [61]. As a 12 degree temperature increase will increase the rate by a further factor of 2, we estimate $k_{\mathrm{dmpo}}=50 \mathrm{Ms}^{-1}$ for the trapping of superoxide by $\mathrm{DMPO}$ at $\mathrm{pH}=7.4$. In the literature there exist a controversy on which radical species is produced by the $\mathrm{NO}$ synthases at $37^{\circ} \mathrm{C}$. It is common experience that attack of DMPO by different radicals may induce formation of spin trap adducts which are identical or indistinguishable by EPR. This feature seriously complicates the identification of the actually produced radical by DMPO. As in our case the adduct formation could be completely abolished by addition of $S O D$, it is proven that superoxide radicals have been trapped. The primary superoxide-DMPO adducts (DMPO-OO / DMPO-OOH) are quite unstable and spontaneously convert into the hydroxyl-DMPO adduct (DMPO-OH) on a timescale of about half a minute (depending on $\mathrm{pH}$ and temperature). Our method therefore quantifies the number of this secondary adduct in the assay. 
This method is based on the usual and reasonable assumption that the yield of secondary adducts is proportional to the number of primary superoxide radicals formed.

\section{Figures}

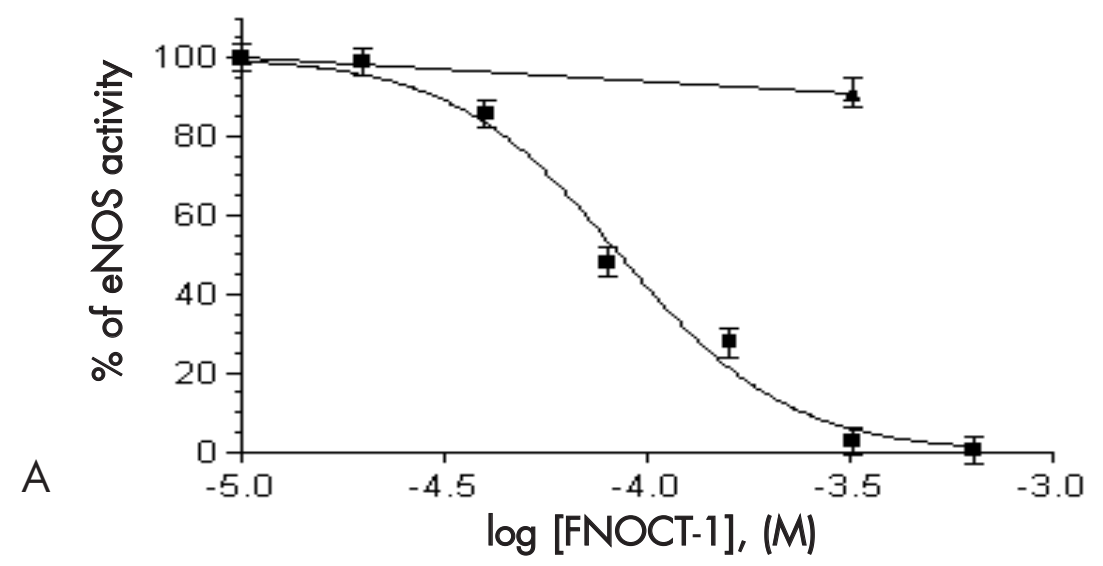

B

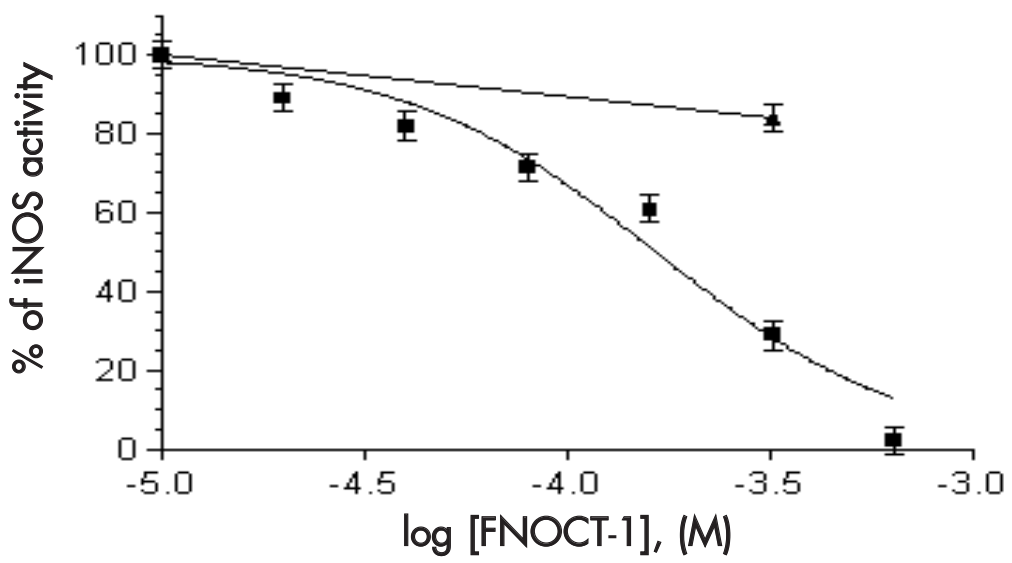

Figure 1

A) Effect of FNOCT-5 on the enzymatic activity of $42 \mu \mathrm{g}$ eNOS in $100 \mu$ Tris buffer $(10 \mathrm{mM}, \mathrm{pH}$ $7.4,37^{\circ} \mathrm{C}$ ). The squares show the inhibition of eNOS by FNOCT-5 in absence of albumin.

Addition of $100 \mathrm{\mu g} \mathrm{ml}^{-1}$ albumin (triangles) suppresses the inhibition.

B) Effect of FNOCT-5 on the enzymatic activity of $57 \mu \mathrm{g}$ iNOS in $100 \mu$ Tris buffer $(10 \mathrm{mM}, \mathrm{pH}$ $7.4,37^{\circ} \mathrm{C}$ ). The squares show the inhibition of iNOS by FNOCT-5 in absence of albumin. Addition of albumin (triangles) cancels the inhibition. 


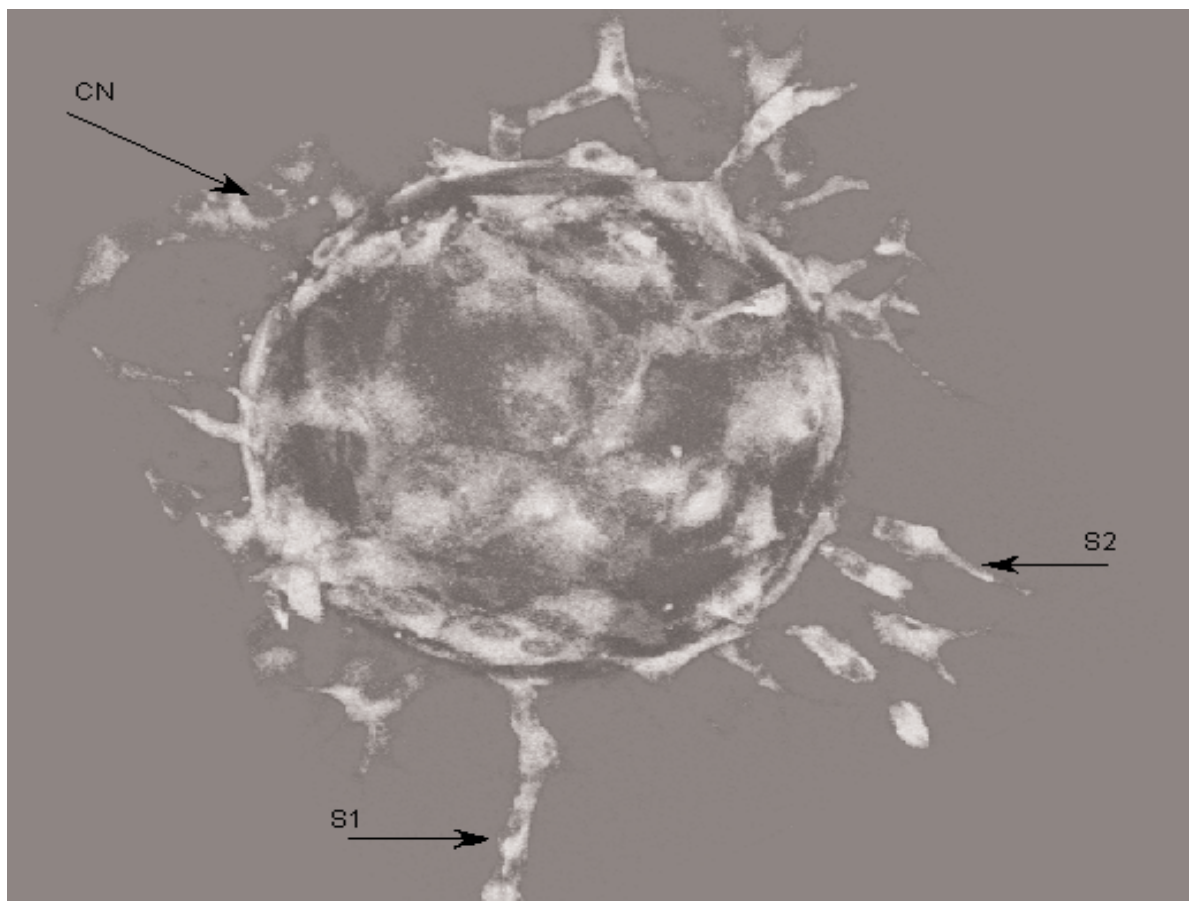

\section{Figure 2}

3D-reconstruction of a typical bead covered with HUVEC's. Image is $300 * 300 * 200 \mu \mathrm{m}(x, y$, z). Clearly visible are the many sprouting cells, either forming vessel-like structures (S1) of single sprouts (S2) pertruding in the fibrin matrix. The FNOCT NO probe is only taken up by cell cytoplasm since the cell nuclei $(\mathrm{CN})$ remain unstained. The probe is not detectable outside the cells. 


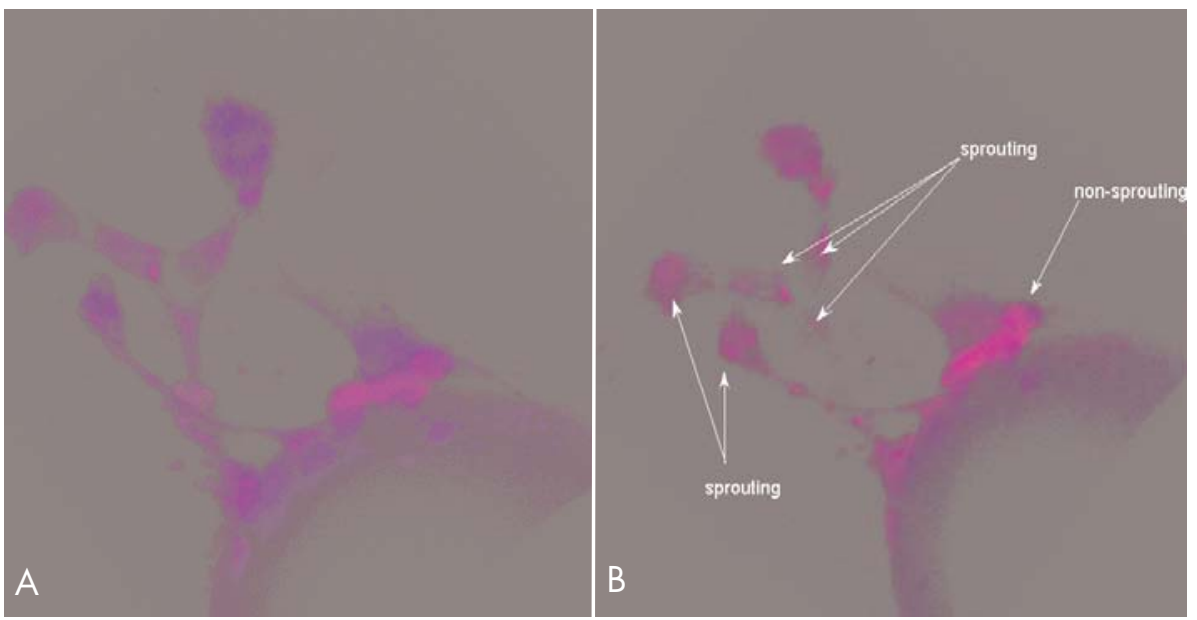

\section{Figure 3}

Typical XY scan (1.5 $\mu \mathrm{m}$ thick slice of $300 * 300 \mu \mathrm{m})$ from a Z-stack image of cultured HUVECs attached to a bead. Visible is a non-sprouting cell, surrounded by sprouting cells growing into the fibrin matrix. Sprouting cells may be distinguished from non-sprouting cells by the prominent tentacular protrusions into the surrounding fibrin matrix. Shown are the fluorescence of a single non-sprouting and various sprouting cells prior to $(A)$ and after (B) calcium ionophore stimulation. Without stimulation, formation of the blue fluorescent FNOCT-4-NOH adduct remained below the detection limit, even 5 hours after accumulation; cell color in a) is the superposition of red fluorescence from accumulated FNOCT-4 traps, and some background blue fluorescence from contamination by the ketone precursor of the FNOCT. After stimulation, the non-sprouting cell maintains its overall purple color, indicative for the absence of NO production, while the NO levels in the sprouting cells increase, as seen from the enhanced blue fluorescence in B) and the increase in $R$ value.

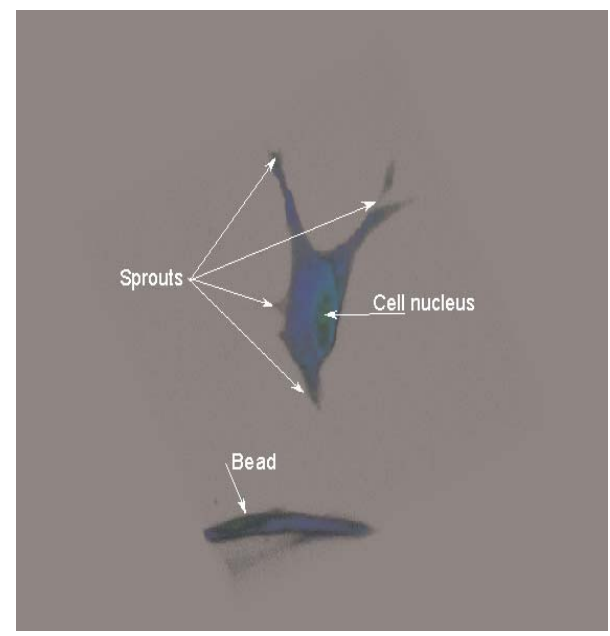

\section{Figure 4}

Typical microscopic 3D-reconstruction of cultured HUVEC attached to a bead, with sprouts growing into the fibrin matrix. Image is $100 * 100 \mu \mathrm{m}$ over a depth of $20 \mu \mathrm{m}$. Image was taken several hours after stimulation with acetylcholine $(50 \mu \mathrm{m})$. The intensity of the blue fluorescence shows that significant amount of the FNOCT-4-NOH adduct has formed in this cell, $R=4$. The blue fluorescence is homogeneously distributed inside the cell. The cell nucleus appears green after addition of SYTO13 at low concentration. 


\section{References}

1. Anggard, E., Nitric oxide: mediator, murderer, and medicine. the Lancet, 1994;343(8907): 1 199-1206.

2. Nathan, C. and Q.W. Xie, Nitric oxide synthases: roles, tolls, an controls. Cell, 1994;78(6):915-918.

3. Andrew, P.J. and B. Mayer, Enzymatic function of nitric oxide synthases. Cardiovasc. Res., 1999;43(3):521-531.

4. Cooke, J.P., NO and angiogenesis. Atheroscler. Suppl., 2003;4(4):53-60.

5. Duda, D.G., D. Fukumura, and R.K. Jain, Role of eNOS in neovascularization: NO for endotheliol progenitor cells. Trends Mol. Med., 2004;10(4):143-145.

6. Murohara, T., T. Asahara, P.A. Hendricks, et al., Nitric oxide synthase modulates angiogenesis in response to tissue ischemia. J. Clin. Invest., 1998;101(1 1):2567-2578.

7. Shimizu, S., M. Kageyama, M. Yasuda, et al., Stimulation of in vitro angiogenesis by nitric oxide through the induction of transcription factor ETS-1. Int. J. Biochem. Cell Biol., 2004;36(1): 114-122.

8. van Faassen, E. and A. Vanin, Nitric oxide: Biological functions and detection. Encyclopedia of Analytical Science. . 2005, Maarssen, NL: Elsevier.

9. von Bohlen und Halbach, O., Nitric oxide imaging in living neuronal tissues using fluorescent probes. Nitric Oxide., 2003;9(4):217-228.

10. Guevara, I., J. Iwanejko, A. Dembinska-Kiec, et al., Determination of nitrite/nitrate in human biological material by the simple Griess reaction. Clin. Chim. Acta., 1998;274(2):177-188.

11. Heyman, S.N. and F. Karmeli, Intrarenal nitric oxide monitoring with a Clark-type electrode: potential pitfalls. Kidney Int., 1997;51(5):1619-1623.

12. Huisman, A., I. Vos, E.E. van Faassen, et al., Anti-inflammatory effects of tetrahydrobiopterin on early rejection in renal allografts: modulation of inducible nitric oxide synthase. FASEB J., 2002;16(9): $1135-1137$.

13. Itoh, Y., F.H. Ma, H. Hoshi, et al., Determination and bioimaging method for nitric oxide in biological specimens by diaminofluorescein fluorometry. Anal. Biochem., 2000;287(2):203209.

14. Joshi, M.S., J.R.j. Lancaster, X. Liu, et al., In situ measurement of nitric oxide production in cardiac isografts and rejecting allografts by an electrochemical method. Nitric Oxide., 2001;5(6):561-565.

15. Nakatsubo, N., H. Kojima, K. Kikuchi, et al., Direct evidence of nitric oxide production from bovine aortic endothelial cells using new fluorescence indicators: diaminofluoresceins. FEBS Lett., 1998;427(2):263-266.

16. Vanin, A.F., A. Huisman, E.S. Stroess, et al., Antioxidant capacity of mononitrosyl-irondithiocarbamate complexes: implications for NO trapping. Free Radic. Biol. Med., 2001;30(8):813-824.

17. Vanin, A.F. and A. Huisman, Iron dithiocarbamate as spin trap for nitric oxide detection: piffalls and successes. Methods Enzymol., 2002;359:27-42. 
18. Meineke, P., U. Rauen, H. de Groot, et al., Nitric oxide detection and visualization in biological systems. Applications of the FNOCT method. Biol. Chem., 2000;381 (7):575-582.

19. Baetz, M., H.G. Korth, P. Meineke, et al., Fluorescence detection of nitric oxide based on cheletropic spin traps. Methods Enzymol., 1999;301:532-539.

20. van Hinsbergh, V.W., A. Collen, and P. Koolwijk, Role of fibrin matrix in angiogenesis. Ann. N Y Acad. Sci., 2001;936:426-437.

21. Douma, K., R.T. Megens, S. Reitsma, et al., Two-photon lifetime imaging of fluorescent probes in intact blood vessels: A window to sub-cellular structural information and binding status. Microsc Res Tech, 2007;70(5):467-75.

22. Megens, R.T., S. Reitsma, P.H. Schiffers, et al., Two-photon microscopy of vital murine elastic and muscular arteries. Combined structural and functional imaging with subcellular resolution. J Vasc Res, 2007;44(2):87-98.

23. Prinzen, L., R.J. Miserus, A. Dirksen, et al., Optical and magnetic resonance imaging of cell death and platelet activation using annexin a5-functionalized quantum dots. Nano Lett, 2007;7(1):93-100.

24. Meineke, P., U. Rauen, H. Groot de, et al., Cheletropic traps for the Fluoresence Spectroscopic Detection of Nitric Oxide (Nitrogen Monoxide) in Biological Systems. Chem. Eur. J., 1999;5:1738-1747.

25. Stroes, E., M. Hijmering, M. van Zandvoort, et al., Origin of superoxide production by endothelial nitric oxide synthase. FEBS Lett., 1998;438(3):161-164.

26. Ulfman, L.H. and D.P. Joosten, IL-8 induces a transient arrest of rolling eosinophils on human endothelial cells. J. Immunol., 2001;166(1):588-595.

27. Presta, A., U. Siddhanta, C. Wu, et al., Comparative functioning of dihydro- and tetrahydropterins in supporting electron transfer, catalysis, and subunit dimerization in inducible nitric oxide synthase. Biochem., 1998;37(1):298-310.

28. Vasquez-Vivar, J., B. Kalyanaraman, P. Martasek, et al., Superoxide generation by endothelial nitric oxide synthase: the influence of cofactors. Proc. Natl. Acad. Sci. USA., 1998;95(16):9220-9225.

29. Meineke, P., Entwicklung eines fluoreszenzspektroskopischen Nachweises von Stickstoffmonoxid fur die Verwendung auf Einzellebene. 1999, Essen, Germany: PhD thesis Essen University.

30. van Zandvoort, M.A.M.J., C.J. de Grauw, H.C. Gerritsen, et al., Discrimination of DNA and RNA in cells by a vital fluorescent probe: lifetime imaging of SYTO 13 in healthy and apoptotic cells. Cytometry., 2002;47:226-235.

31. Burtis, C., Tietz textbook of clinical chemistry. 1999, Philadelphia: W.B.Saunders.

32. Gillham, B.P.D. and J.H. Thomas, Biochemical basis of medicine. 1997, Oxford, UK: Butterworth-Heinemann.

33. Galley, H.F., B.E. Walker, P.D. Howdle, et al., Regulation of nitric oxide synthase activity in cultured human endothelial cells: effect of antioxidants. Free Radic. Biol. Med., 1996;21(1):97-101. 
34. Khadour, F.H., D. Panas, P. Ferdinandy, et al., Enhanced NO and superoxide generation in dysfunctional hearts from endotoxemic rats. Am. J. Physiol. Heart Circ. Physiol., 2002;283(3):H1 108-1115.

35. McCord, J.M., Oxygen-derived free radicals in postischemic tissue injury. N. Engl. J. Med., 1985;312(3):159-163.

36. Zweier, J.L., R. Broderick, P. Kuppusamy, et al., Deternination of the mechanism of free radical generation in human aortic endothelial cells exposed to anoxia and reoxygenation. J. Biol. Chem., 1994;269(39):24156-24162.

37. Kojda, G. and D. Harrison, Interactions between NO and reactive oxygen species: pathophysiological importance in atherosclerosis, hypertension, diabetes and heart failure. Cardiovasc. Res., 1999;43(3):562-571.

38. Goldstein, S. and G. Czapski, The reaction of NO. with O2.- and HO2.: a pulse radiolysis study. Free Radic. Biol. Med., 1995; 19(4):505-510.

39. Huie, R. and S. Padmaja, The reaction of NO with superoxide. Free Radic. Res. Commun., 1993;18(4):195-199.

40. Kissner, R., T. Nauser, P. Bugnon, et al., Formation and properties of peroxynitrite as studied by laser flash photolysis, high-pressure stopped-flow technique, and pulse radiolysis. Chem. Res. Toxicol., 1998;11:1285-1292.

41. Beckman, J.S. and W.H. Koppenol, Nitric oxide, superoxide, and peroxynitrite: the good, the bad and ugly. Am. J. Physiol., 1996;271 (Pt 1):C1424-1437.

42. Muijsers, R.B., G. Folkerts, P.A. Hendricks, et al., Peroxynitrite: a two-faced metabolite of nitric oxide. Life Sci., 1997;60(21):1833-1845.

43. Stenert, V., Synthese o-chinoider Fluorescenzmarker fuer Stickstoffmonoxid und deren Anwendung in chemischen Systemen mit biologischer Relevanz. 2003, Essen, Germany: PhD thesis Universitaet Essen.

44. Martin, A., M.R. Komada, and D.C. Scane, abnormal angiogenesis in diabetes mellitus. Med. Res. Rev., 2003;23(2):117-145.

45. Duan, J., T. Murohara, H. Ikeda, et al., Hyperhomocysteinemia impairs angiogenesis in response to hindlimb ischemia. Arterioscler. Thromb. Vasc. Biol., 2000;20(12):2579-2585.

46. Duan, J., T. Murohara, H. Ikeda, et al., Hypercholesterolemia inhibits angiogenesis in responsse to hindlimb ischemia: nitric oxide-dependent mechanism. Circulation., 2000; 102(19 Suppl 3):III370-376.

47. Jang, J.J., H.K. Ho, H.H. Kwan, et al., Angiogenesis is impaired by hypercholesterolemia: role of asymmetric dimethylarginine. Circulation., 2000;102(12):1414-1419.

48. Brzozowski, T. and S.J. Konturek, Healing of chronic gastric ulcerations by L-arginine. Role of nitric oxide, prostaglandins, gastrin and polyamines. Digestion., 1995;56(6):463-471.

49. Leibovick, S.J., P.J. Polverini, T.W. Fong, et al., Production of angiogenic activity by human monocytes requires an L-arginine/nitric oxide-synthase-dependent effector mechanism. Proc. Natl. Acad. Sci. USA., 1994;91(10):4190-4194.

50. Babaei, S., K. Teichert-Kuliszewska, J.C. Monge, et al., Role of nitric oxide in the angiogenic 
response in vitro to basic fibroblast growth factor. Circ. Res., 1998;82(9):1007-1015.

51. Ridnour, L.A., J.S. Isenberg, M.G. Espey, et al., Nitric oxide regulates angiogenesis through a functional switch involving thrombospondin. Proc. Natl. Acad. Sci. USA., 2005;102(37):13147-13152.

52. Bouloumie, A., V.B. Schini-Kerth, and R. Busse, Vascular endothelial growth factor upregulates nitric oxide synthase expression in endothelial cells. Cardiovasc. Res., 1999;41 (3):773-780.

53. Kon, K., S. Fujii, H. Kosaka, et al., Nitric oxide synthase inhibition by N(G)-nitro-L-arginine methyl ester retards vascular sprouting in angiogenesis. Microvasc. Res., 2003;65(1):2-8.

54. Papapetropoulos, A.G. and G. Garcia-Cardena, Nitric oxide production contributes to the angiogenic properties of vascular endothelial growth factor in human endothelial cells. J. Clin. Invest., 1997;100(12):3131-3139.

55. Ziche, M., L. Morbidelli, R. Choudhuri, et al., Nitric oxide synthase lies downstream from vascular endothelial growth factor-induced but not basic fibroblast growth factor-induced angiogenesis. J. Clin. Invest., 1997;99(1 1):2625-2634.

56. Lee, P.C., A.N. Salyapongse, G.A. Bragdon, et al., Impaired wound healing and nagiogenesis in eNOS-deficient mice. Am. J. Physiol., 1999;277(4 Pt 2):H1600-1608.

57. Weis, M., C. Heeschen, A.J. Glassford, et al., Statins have biphasic effects on angiogenesis. Circulation., 2002;105(6):739-745.

58. Jerkic, M., A. Rodriguez-Barbero, M. Prieto, et al., Reduced angiogenic response in adult endoglin heterozygous mice. Cardiovasc. Res., 2006;69:845-854.

59. Lancaster, J.R., Jr., A tutorial on the diffusibility and reactivity of free nitric oxide. Nitric Oxide, $1997 ; 1(1): 18-30$.

60. Sbaa, E., F. Frerart, and O. Feron, The Double Regulation of Endothelial Nitric Oxide Synthase by Caveolae and Caveolin: A Paradox Solved Through the Study of Angiogenesis. Trends Cardiovasc. Med., 2005;15(5):157-162.

61. Finkelstein, E., G.M. Rosen, and E.J. Raukmann, Spin trapping of superoxide and hydroxyl radical: practical aspects. Arch. Biochem. Biophys., 1980;200(1):1-16. 
Chapter 6 


\section{Chapter 7}

\section{In Vivo Imaging of Murine Large Arteries using Two-Photon Laser Scanning Microscopy: a Feasibility Study}

R.T.A. Megens ${ }^{a}$; M.G.A. oude Egbrink ${ }^{b}$; W. Engels ${ }^{a}$; P.J.A. Leenders ${ }^{c}$; E. J. L. Brunenberg ${ }^{\text {d; }}$ B.J.A. Janssen ${ }^{\text {c; }}$ B.M. ter Haar Romeny ${ }^{\text {d; D.W. Slaaf }}{ }^{\text {a d }}$; M.A.M.J. van Zandvoort ${ }^{a}$.

Departments of 'Biophysics, 'Physiology, and 'Pharmacology and Toxicology, Cardiovascular Research Institute Maastricht, Maastricht University, Maastricht, The Netherlands. 'Department of Biomedical Engineering, Eindhoven University of Technology, Eindhoven, The Netherlands. 


\section{Abstract}

In vivo imaging of especially large arteries using two-photon laser scanning microscopy (TPLSM) results in images (optical sections) that severely suffer from motional artifacts within each image and between subsequent images. We applied TPLSM image acquisition triggered on cardiac and respiratory cycle, and accelerated image acquisition in order to reduce the impact of motion between subsequent images and within each image.

Carotid arteries of anesthetized C57BL6/J mice $(n=4)$ and renal arteries of anesthetized WKY-rats $(n=6)$ were surgically exposed, labeled with specific fluorescent markers for cell nuclei and cytoplasm, and imaged using TPLSM. Blood pressure and respiratory signals were recorded and fed into a trigger unit. The trigger unit generated a trigger pulse at a stable moment in the cardiac and respiration cycle which was used to acquire single images. Image acquisition was accelerated by reducing the number of scanned pixels or reduction of pixel dwell times (or a combination of both). Spatial resolution and signal-to-noise $(S / N)$ ratio are reduced at higher image acquisition speeds. Therefore, filtering in the Fourier domain was examined to improve overall image quality.

In both mice and rats, timing of the trigger moment at the diastolic phase in the cardiac cycle combined with acquisition rates of $4 \mathrm{~Hz}$ or higher, resulted in stable images with reduced motional artifacts. Moreover, subsequently acquired images contained a similar and stable image of the artery. Application of the Fourier transformation further improved overall image quality.

We have shown the feasibility of stable imaging of large arteries using TPLSM triggered on cardiac and respiratory cycle. The presented method creates new opportunities for in vivo studying of various structural and functional properties of the (diseased or damaged) arterial wall at subcellular level. 


\section{Introduction}

Recently, we have performed ex vivo imaging at cellular level inside the vessel wall of large murine arteries in isolated vessels mounted in a perfusion chamber $[1,2]$. Fluorescent dyes were used to highlight structural or functional aspects of interest using two-photon laser scanning microscopy (TPLSM). This setup allows for application of physiological transmural pressure and perfusion. In vivo imaging of such arteries at cellular level, using TPLSM, is hampered by motional artifacts caused by heartbeat (up to 600 beats per minute in anesthetized mice, causing local tissue motion, and pulsation of the artery) and respiration. Such motional disturbances strongly reduce image quality and therefore have to be overcome.

In conventional fluorescence microscopy, the whole field of view is illuminated and instantaneously imaged at video rate and higher $(50-500 \mathrm{~Hz})$, with a limited depth of field $(1-10 \mu \mathrm{m})$ [3]. Fluorescently labeled structures, however, may be visible even if they are more than $10 \mu \mathrm{m}$ out of focus. When focusing on the luminal endothelial cell layer of a blood vessel, this technique is therefore particularly suitable for visualization of blood cell-vessel wall interactions [4, 5] and hemodynamic parameters [6] especially in thin transparent tissues where detection of fluorescent blood cells is possible at a depth up to $100 \mu \mathrm{m}$. Although it has been demonstrated that conventional intravital fluorescence microscopy allows for detection of fluorescently labeled blood cells that interact with the vessel wall in large (atherosclerotic) arteries [79], its applicability for imaging of structures in the vessel wall of such arteries at a subcellular level is limited. Image quality is hampered by contributions from out-of-focus fluorescence and rapidly decreases at deeper layers due to scattering. These strong scattering properties of the wall of larger arteries preclude the use of conventional intravital fluorescence microscopy to visualize subcellular structures.

Confocal laser scanning microscopy (CLSM) limits the contribution of out-offocus fluorescence light and offers excellent images of vascular structures in thin and transparent tissues [3, 10]. However, when imaging in thicker, scattering tissues, such as the wall of large arteries, scattering of the emission light strongly increases with depth and as a consequence, the spatial resolution deep in the sample is reduced [11].

Two-photon laser scanning microscopy (TPLSM) does allow ex vivo imaging of large arteries at high spatial resolutions [1, 2, 11-14] and in vivo visualization of various relatively stationary tissues and structures such as brain [15], kidney [16-18], microvasculature [19], and lymphatics [20]. 
Since two-photon excitation of fluorescent molecules only occurs in a small volume element (voxel) in the focal plane, scattering of the emission light in the tissue does not affect the spatial resolution. Indeed, any light reaching the detector can originate only from the excited voxel. In TPLSM the wavelength of the excitation light is almost double of that in conventional microscopy. While this limits the resolution, especially at low depths in the tissue, this effect is counterbalanced at higher depth by significantly reduced scattering of longer wavelengths. Conclusively, TPLSM combines large penetration depth, good optical sectioning, and subcellular resolution.

Unfortunately, TPLSM does not allow image acquisition at video rate since image composition is based on point-by-point scanning through the optical section, as in all laser scanning microscopes. Therefore, imaging of large arteries in vivo is complicated since the acquired images will suffer from motional artifacts (introduced by the cardiac and respiratory cycle of the living animal) within single images and between subsequent images.

Motional artifacts may be minimized by making several adaptations to the acquisition mode: (1) Acquisition of each separate image in a time sequence should start at a specific, fixed moment in the cardiac- and respiration cycle. This potentially results in a series of images that contain a similar part of the (still moving) arterial wall. (2) Acceleration of image acquisition will decrease motional artifacts within each separate image. This can be achieved by reducing the number of pixels, or the pixel dwell times for a combination of both). Unfortunately, accelerated image acquisition also results in a reduction of either in spatial resolution or signal-to-noise $(S / N)$ ratio.

(3) When all acquisition parameters are optimal, application of specific image analysis tools might increase overall image quality and might allow removal of remaining motional artifacts within each image.

In the pilot study described in this chapter, we tested the effects of image acquisition triggered on heart rate (arterial blood pressure) and respiration in combination with accelerating sampling rates for in vivo TPLSM imaging at a subcellular level. Furthermore, image analysis routines were utilized to enhance image quality and to correct for errors that occurred as a consequence of accelerated image acquisition. Imaging was performed in surgically exposed carotid arteries of C57BL6/J mice and renal arteries of WKY rats. Arteries were stained with vital and specific fluorescent markers for elastin bands and cell nuclei. Collagen in the tunica adventitia was visualized by using the nonlinear scattering process second harmonic generation (SHG) [11, 21-23]. Additionally, cytoplasm of (blood) cells and blood platelets were fluorescently labeled to visualize their interaction with the vessel wall. The advantages and 
disadvantages of the method will be discussed and criteria for timing of the trigger pulse will be provided.

\section{Materials and Methods}

\section{Animals}

All experiments were in line with institutional guidelines and approved by the local ethics committee on the use of laboratory animals. Mice (C57BL6/J; n=4) were anesthetized using subcutaneous administration of a mixture of $75 \mathrm{mg} / \mathrm{kg}$ ketamin (Nimatek, Eurovet, Cuijck, the Netherlands) and $15 \mathrm{mg} / \mathrm{kg}$ xylazin (Xylazin, Ceva Sante Animale BV, Naaldwijk, the Netherlands) in a volume of $3 \mathrm{ml} / \mathrm{kg}$. Anesthesia was maintained by subcutaneous injection of $0.88 \mathrm{ml} / \mathrm{kg}$ of the same xylazin-ketamin mixture every 30 minutes. Rats (WKY; $n=6$ ) were anesthetized by a single intraperitoneal injection of urethane $(1.7 \mathrm{mg} / \mathrm{kg})$. All anesthetics were dissolved in saline $10.9 \% \mathrm{NaCl}$ solution; Braun Melsungen AG, Melsungen, Germany). Body temperature was monitored using a rectal probe (Watlow, PT100 sensor, UK) and maintained at approximately $37^{\circ} \mathrm{C}$ by a heating platform (TH60-SMZ, Linkam Scientific Instruments, England).

\section{Tissue preparation and instrumentation}

Animals were placed in supine position on the heating platform mounted on the microscope stage. In mice, the right common carotid artery was surgically exposed up to the bifurcation [7]. In rats, the abdominal region was surgically opened and the left renal artery exposed. In order to simplify discrimination between artery and surrounding tissues, a thin black plastic sheet was placed underneath artery segments in both preparations. All exposed areas were kept moist during the remainder of the experiment by dripping natriumchloride 0.9 \% (Braun Melsungen AG, Melsungen, Germany) on it.

For administration of fluorescent dyes, in both species the left jugular vein was cannulated with a catheter made of polyethylene PE 10 tubing. Blood pressure was measured using a catheter (PE 10) in the left femoral artery linked to two blood pressure sensors (Baxter uniflow, Baxter B.V., Utrecht, the Netherlands); the first was used to monitor absolute blood pressure and, hence, the condition of the animal. The second blood pressure signal was used as input in the trigger unit which only uses the first derivative, i.e. changes in blood pressure signal. All catheters and blood pressure probes were filled with a mixture of saline and heparin $(1 \mathrm{\mu l} / \mathrm{ml})$.

In order to stabilize respiration, the trachea of the mice was intubated using a blunt needle (1.2 * $40 \mathrm{~mm}$ ) (in rats using PE 200 tubing) and ventilated with normal air using a ventilator (mouse: respiration rate $4 \mathrm{~Hz}$, Minivent 845, Hugo Sachs Electronic GmbH, Germany; rat: respiration rate 2 Hz, Harvard 
rodent respirator, Harvard Apparatus, Massachusetts, USA). A respiration sensor (Graseby, Wicklow, Ireland) was placed on top of the chest in order to obtain a (relative) respiration signal for the trigger unit.

\section{Triggering}

The respiration sensor and one of the blood pressure sensors was connected to the trigger unit (Rapid Biomedical, Würzburg, Germany), originally designed for NMR tomography. The output trigger signal was coupled to the trigger/synchronizer input of the instrumentation control unit of the TPLSM system. The trigger unit displayed both the respiration signal and the relative blood pressure signal. By setting thresholds for both signals, a stable time window in the cardiac and respiration cycle could be defined. The blood pressure signal was the primary determinant for the trigger moment. Note that the stability of the obtained blood pressure signals was occasionally hampered by leakage of the fluid filled blood pressure sensors. Since blood pressure is known to vary with respiration, the respiration signal was used to find the time window in which blood pressure pulses were optimally stable. At the start of such a (selected) time window, a trigger pulse started the acquisition of a single optical section. A trigger delay for both blood pressure and respiration could be set in order to simplify the search for an optimum trigger moment in the cardiac and respiration cycle without the need for adjustment of the actual blood pressure signal, respiration signal, or trigger thresholds. As a result, a series of triggered optical sections in time was obtained that contained similar parts of the (moving) sample.

For analyses of the triggering and acquisition timing, the trigger pulse, blood pressure signal, and respiration signal originating from the trigger unit, and absolute blood pressure signal (originating from the first sensor) were digitally recorded using a programmable acquisition system $M-P A Q$ in combination with the programmable acquisition software package IDEEQ (IDEE, Maastricht, the Netherlands). Sampling rate was $1000 \mathrm{~Hz}$. Total frame time, start time of each subsequent image, and time gap between two subsequent optical sections (frames) were recorded using the TPLSM acquisition software (Biorad/Zeiss Lasersharp 6.0; Hemel Hempstead, GB).

\section{Fluorescent staining}

Nuclei of viable cells were labeled with the viable cell membrane permeable DNA / RNA marker SYTO13 (2.0 $\mu$ M; $\lambda_{\text {max emission }}=510 \mathrm{~nm}$ ) (Molecular Probes, Leiden, the Netherlands). Although elastin displays an autofluorescence signal [23], the autofluorescence is weak when compared to 
the fluorescence of the probes. Therefore, eosin $10.5 \mu \mathrm{M} ; \lambda_{\text {max emission }}=545$ $\mathrm{nm}$; Molecular Probes) was used as a specific and strongly fluorescent elastin marker [24]. SYTO13 and eosin were dissolved in saline and administered topically on the artery. In four rats, SYTO13 was infused intravenously $10.1 \mathrm{ml}$ of a $4.0 \mu \mathrm{M}$ solution in saline) in order to more specifically stain the endothelial cell nuclei and blood cells. Circulating blood platelets, cytoplasm of leukocytes, and cells in the arterial wall were labeled fluorescently by intravenous administration of acridin red (AR; Chroma-Gesellschaft Schmid GmbH, Germany); $0.03 \mathrm{ml}$ in mice or $0.1 \mathrm{ml}$ in rats of a $2.0 \mathrm{mg} / \mathrm{ml}$ solution in saline with $5 \%$ ethanol. Collagen was visualized by second harmonic generation or SHG $\left(\lambda_{\text {excitation }}=840 \mathrm{~nm}, \lambda_{\text {max emission }}=420 \mathrm{~nm}\right)$.

\section{Image acquisition}

\section{Microscope}

To accommodate the animal preparations, the workspace between the microscope stage and objective lenses of our Nikon E600FN microscope (Nikon Corporation, Tokyo, Japan) had to be increased: the standard stage (2.9 cm thick) was replaced by a home-built microscope stage $10.8 \mathrm{~cm}$ thick, IDEE, Maastricht, the Netherlands). Adjustment of the xy-position of the in vivo stage was performed with a micromanipulator (Leitz micromanipulator $M$, Wetzlar, Germany). The heating stage was mounted on top of the microscope stage.

A Nikon E600FN microscope (Nikon Corporation, Tokyo, Japan) was connected to a Biorad 2100MP multiphoton system (Biorad, Hemel Hempstead, GB). The excitation source was a 140 femtosecond pulsed Ti: sapphire laser (Spectra Physics Tsunami, Mountain View, USA) tuned and mode-locked at either $800 \mathrm{~nm}$ (for visualization of fluorescent probes) or 840 $\mathrm{nm}$ (to visualize collagen by second harmonic generation). Laser light reached the sample through the microscope objective $160 \mathrm{X}$ water dipping, numerical aperture 1.0, working distance $2 \mathrm{~mm} ; 40 \mathrm{X}$ water dipping, numerical aperture 0.8 , working distance $2 \mathrm{~mm} ; 20 \mathrm{X}$ water dipping, numerical aperture 0.5, working distance $2 \mathrm{~mm}$ ).

Three photo multiplier tubes (PMTs) were used to detect the emitted fluorescent signals. For imaging of each of the fluorescent markers, PMTs were tuned corresponding to parts of the emission spectra of the fluorescent markers used: SYTO 13, 500-540 nm (PMT 2); Eosin, 530-560 nm (PMT 2) and / or 560-590 nm (PMT 3); AR, 560-600 nm (PMT 3); SHG, 400-450 nm (PMT 1). For simultaneous imaging of a combination of fluorescent markers, each PMT was tuned for minimal bleed-through of the fluorescent markers used. To prevent photochemical and thermal damage, laser power was kept as low as possible [25]. 
From each PMT, separate images of 256 * 256 pixels were obtained and combined into one RGB-image. Series of 20-100 subsequent xy-images were recorded (time-series). Acquisition of each separate image was started by the trigger pulse derived from the trigger unit. Optimal frame rate was determined empirically by adjusting the line speed until the image contained only little motional disturbances. Frame rates varied from $2.3 \mathrm{~Hz}$ to $9.4 \mathrm{~Hz}$, dependent on matrix size, pixel dwell time, and line speed. Note that the mentioned frame rates account for acquisition of one single image. Acquisition of a series of subsequent images can only be performed at a maximum acquisition time of approximately 1 frame per second (due to a delay within the hardware of the microscope system and triggering on respiration). The high frame rates which are necessary for in vivo imaging automatically apply additional optical zoom in order to reduce total scan area, and hence, total acquisition time (table 1). As an advantage, spatial resolution is increased.

For the initial detection of the artery, the $20 \mathrm{X}$ water dipping objective was used in combination with a $20 \mathrm{~W}$ halogen cold light source (Schott KL 200, Schott AG, Mainz, Germany).

Table 1 image acquisition characteristics; line scan rate (lines per second; lps) in relation to maximum size of the field of view (f.o.v.).

\begin{tabular}{llllll}
\hline & $\begin{array}{l}\text { Normal scan } \\
\text { rate }\end{array}$ & $2 \mathrm{X}$ scan rate & $4 \mathrm{X}$ scan rate & $\begin{array}{l}\text { Automatically } \\
\text { applied zoom }\end{array}$ & $\begin{array}{l}\text { Max } \\
\text { f.o.v. }\left(\mu \mathrm{m}^{2}\right)\end{array}$ \\
\hline Line scan rate(lps) & 25 & 50 & 100 & 1.0 & $206^{2}$ \\
& 50 & 100 & 200 & 1.0 & $206^{2}$ \\
& 166 & 332 & 664 & 1.0 & $206^{2}$ \\
& 500 & 1000 & 2000 & 1.0 & $206^{2}$ \\
& 600 & 1200 & 2400 & 1.2 & $172^{2}$ \\
& 750 & 1500 & 3000 & 1.5 & $137^{2}$ \\
& 1200 & 2400 & 4800 & 1.5 & $137^{2}$ \\
& 1500 & 3000 & 6000 & 1.5 & $137^{2}$ \\
\hline Protocol & 1800 & 3600 & 7200 & 1.64 & $126^{2}$ \\
\hline
\end{tabular}

\section{Image processing}

Image reconstructions were performed using the Image-Pro Plus 6.0 software (Media Cybernetics Inc., Silver spring, USA). Image processing was performed using a software tool which uses the Mathematica 5.1 software package with "Digital Image Processing", "Front-end Vision" and "Mathvision tools" plugins (Wolfram Research Inc., Champaign, IL, USA) as a platform. This tool was 
developed in collaboration with the department of Biomedical Engineering Eindhoven, the Netherlands, for motion correction and to improve image quality by application of correction of interlacing inaccuracy and filtering in the frequency domain (Fourier transformation and analysis).

\section{Results and Discussion}

\section{Non-triggered imaging}

Ex vivo imaging of (non-moving) large arteries mounted in a perfusion chamber, is typically performed with an acquisition rate of $0.1 \mathrm{~Hz}$ and a matrix size of $512 * 512$ pixels $[1,2]$. The resulting optical sections have a good $\mathrm{S} / \mathrm{N}$-ratio and high spatial resolution (up to $0.3 * 0.3 * 1.2 \mu \mathrm{m}$, dependent on optical settings). During in vivo imaging, however, images should be acquired well within one cardiac cycle. In mice anesthetized with ketamin-xylazin mixture, the mean duration of one cardiac cycle was $0.24 \mathrm{~s}(4.2 \mathrm{~Hz})$. This fairly low cardiac cycle for mice is a consequence of the applied anesthetic agents ketamin-xylazin which cause a strong depression of the heart rate in mice $(<55 \%$ ) [26]. In rats, anesthetized with urethane (which only has minor effects on heart rate), the average cardiac cycle duration was $0.17 \mathrm{~s}(5.9 \mathrm{~Hz})$. In order to limit motional distortions, imaging frequencies should therefore be higher than $4 \mathrm{~Hz}$. Such high frame rates can be achieved either by increasing the line scan rate or decreasing the number of lines per image (matrix size). The first method, however, decreases the pixel dwell time at the cost of a lower $\mathrm{S} / \mathrm{N}$-ratio and, at higher rates, is accompanied by an automatic optical zoom with consequently a smaller field of view. The second method results in lower pixel resolution, which can only be resolved by using an equivalent optical zoom.

Comparison of several acquisition rates for imaging of an ex vivo mounted artery revealed that imaging at a rate of $3.0 \mathrm{~Hz}$ yielded optical sections with a sufficient large field of view (no optical zoom is induced) and relatively good overall image quality. However, this frequency was not high enough for in vivo imaging since acquisition time exceeded a complete cardiac cycle and therefore all motions within this cycle were captured in the image (fig. 1). The largest movements of the vessel wall occur during the systolic phase of the cardiac cycle.

An in vivo acquisition rate of approximately $9 \mathrm{~Hz}$ further reduced the $\mathrm{S} / \mathrm{N}$-ratio and field of view. The overall image quality was still acceptable. When acquiring a series of subsequent images, the tissue visualized within each optical section was different since the start of acquisition occurred randomly within the cardiac cycle (fig. 2). Most optical sections were of low quality due 
to movements of the sample, loss of focus on the sample, or even complete disappearance from the field of view. However, some of the optical sections of such series of subsequent images contained tissue with relatively little motional disturbances. Indeed, the advantage of such relatively high acquisition rates is the possibility of acquiring an image well within one cardiac cycle. This implies that some of the optical sections may be fully acquired within the duration of the diastolic phase where movement of the artery is limited. This removes large motional artifacts from at least some of the optical sections (fig. 2c, d) and is indicative of the fact that proper timing of the start of image acquisition may lead to relatively stable and repeatable images.

\section{Triggered in vivo imaging}

Controlled triggered image acquisition of each optical section (in a series of 20-100 subsequent images) resulted in subsequent images that all display the same part of the artery during the same phase of movement and are, thus, better comparable.

Triggered imaging at higher acquisition rates (fig. 3-4; acquisition rate 6-7 Hz, yielded better results than at rates in the order of one cardiac cycle (fig. 5; acquisition rate $4 \mathrm{~Hz}$ ). In the former case, the image could be acquired in a shorter part of the cardiac cycle and, therefore, a more stable period could be found.

The importance of exact timing of the trigger moment was tested empirically at various moments in the cardiac cycle. Signal analysis (in mice) revealed that series of subsequent optical sections with smallest impact of motional disturbances were obtained when the trigger moment was set approximately 60-70 ms before the end-diastolic value of the pressure wave. Since the total delay between generation of the trigger pulse and start of image acquisition in our setup was constant and approximately 200 ms, image acquisition actually started at peak pressure (i.e. the beginning of the diastolic phase). In rats, triggered imaging often resulted in more stable images and smaller motional disturbances than in mice, probably because of the somewhat lower motional effects in the renal artery (further away from the heart). Timing of image acquisition in (part of) the systolic phase of the heart cycle resulted in images with large motional artifacts and strong deformations of the tissue, even at higher imaging rates.

We have shown the feasibility of in vivo imaging of several structures in the arterial wall, and even detection of some blood cells adhering to or slowly moving past the endothelium. However, in vivo imaging of large arteries using TPLSM triggered on blood pressure and respiration still has an important 
limitation. Accurate tracking of (slowly) moving cells, as visible in figure 4, is not possible because time intervals between two images are relatively long (approximately $1.0 \mathrm{~s}$ ) and more importantly, rather variable, due to the stroboscopic-like acquisition subsequent images in a triggered series. This stroboscopic-like acquisition (caused by the delay of the microscope hardware and triggering on respiration) is a major limitation for determination of functional properties such as imaging of calcium or nitric oxide dynamics or tracking of moving objects such as blood cells rolling along the endothelium.

The presented results of in vivo imaging may be improved in two ways: (1) the used TPLSM setup only has a useful acquisition rate of $10 \mathrm{~Hz}$ and total acquisition time (temporal resolution) of approximately $1 \mathrm{~Hz}$, and does not allow imaging at video rates $(50-100 \mathrm{~Hz})$. High speed two-photon microscopes $[19,27]$ that enable image acquisition at video rates may be better suited for in vivo imaging of strongly moving objects. Furthermore, narrowing the pulsewidth of the excitation light will enhance the probability of the two-photon excitation [28]. As a consequence, signal strength and, thus, S/N-ratio increases. Thus is beneficial for the image quality of accelerated image acquisition. (2) Application of the more sophisticated solid state pressure transducer catheter (Miller transducer without fluid column, as applied in [29]) yields more stable and accurate blood pressure signals and may enhance the precision of triggering and, thus, provide more accurate timing of image acquisition.

\section{Image analysis}

Overall image quality suffers from fast acquisition rates that affect the $S / N$ ratio, due to short pixel dwell times (fig. 7a). Moreover, fast image acquisition at doubled or quadrupled line scan rates (table 1) resulted in images that appeared to be stretched in horizontal direction. This error is a consequence of inaccurate build-up of the images at higher acquisition speeds due to a change in the scanning protocol. In order to reduce total acquisition time, odd scan lines of the matrix are scanned from left to right and even scan lines are scanned from right to left, instead of scanning each horizontal line from left to right (as is standard scanning mode with lower acquisition rates). Image analysis of such images revealed that in comparison with the odd scan lines, the even scan lines of the image matrix were shifted three pixels in horizontal direction, causing the smeared look of the images. Correction of this inaccuracy resulted in images that appear sharper (fig. 7b).

In order to further improve overall image quality of these corrected images, Fourier transformation was performed to discriminate the original image 
frequencies (that lie around the origin, i.e. in the corners of the periodic spectrum plot) from higher noise and interlacing frequencies. The undesirable higher frequencies were then filtered out by multiplying the Fourier-spectra with a mask based on a 2D-Gaussian weighted function. As a result, S/N-ratio is improved (fig. 7c).

\section{Conclusion}

The presented method creates new opportunities for in vivo studying of various structural and functional properties of the (diseased or damaged) arterial wall at a subcellular level. It holds potential for in vivo visualization of the relationship between various structures and their functions in changing situations. Furthermore, three dimensional imaging of the arterial wall might be possible in the near future, especially when more sophisticated solid-state blood pressure transducers will be used yielding a more stable blood pressure signal. In addition, it may provide an important tool for in vivo testing and validation of novel molecular agents such as CNA35/OG488 [2] or targeted quantum dots [30], as it provides detailed information regarding the behavior of such agents in large arteries in vivo.

In the near future, triggered TPLSM imaging will be applied for in vivo visualization of various properties of the arterial wall such as nitric oxide production, the relation between adhesion molecules and adhering of blood cells to the endothelium or diapedesis of blood cells into the diseased arterial wall.

\section{Acknowledgements}

We thank Koen Reesink and Evelien Hermeling from Maastricht University, Department of Biophysics, for their help with analyzing blood pressure and respiration signals. 


\section{Figures}
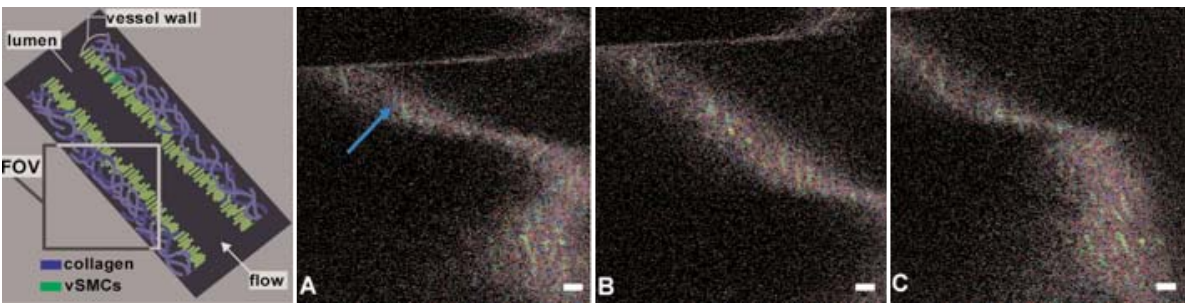

Figure 1

Three subsequent optical sections (A-C) of a left carotid artery of a C57Bl6/J mouse obtained in vivo without application of external triggering. Frame rate was $2.3 \mathrm{~Hz}(1200 \mathrm{lps}$; line scan rate 1 $X$, matrix size $512 * 512$ pixels). Drawing indicates the position of the optical sections in the vessel wall; bars indicate $20 \mu \mathrm{m}$. Cell nuclei (SYTO13, green) and collagen (SHG, blue/purple) are visible. All sections are disturbed by motional artifacts which cause the arterial wall (blue arrow) to appear as a curved-like structure. Moreover, every optical section contains different parts of the (moving) vessel wall. The vSMC nuclei (green), with their typical morphology and orientation, are hardly recognizable; the position of the lumen is unclear.

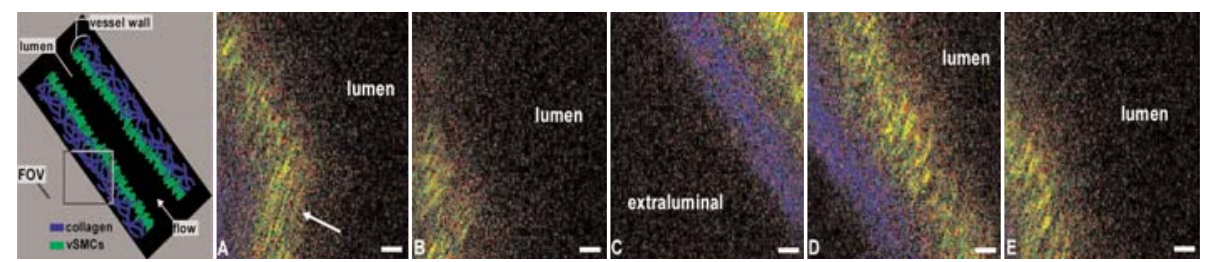

Figure 2

Five successive optical sections of a left carotid artery of a C57BL6/J mouse in vivo without triggering at a frame rate of $9.4 \mathrm{~Hz}(2400 \mathrm{lps}$; line scan rate $2 \mathrm{X} ; 256$ * 256 pixels). Note the reduced image size as a consequence of the high frame rate. Drawing indicates the position of the optical sections; bar $=20 \mu \mathrm{m}$. Cell nuclei (SYTO13, green) and collagen (SHG, blue) in the arterial wall are visible. Due to motion, the visualized part of the artery is different in every subsequent image. Some optical sections (C, D) contain recognizable parts of the arterial wall and, thus, appear to be imaged in a stable moment of the heart cycle. However, in some regions /white arrow), motional disturbances cause alteration of vSMCs morphology which appear to be stretched $(A, B)$. Luminal and abluminal side of the artery are recognizable due to the clearly observable collagen (blue) in the tunica adventitia. 

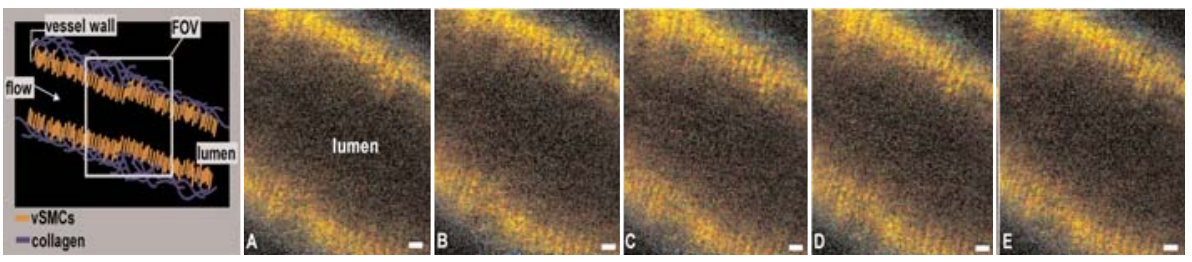

\section{Figure 3}

Five subsequent optical sections (A-E) of the left renal artery of a WKY-rat in vivo obtained with triggered acquisition at a single depth. Frame rate was $7 \mathrm{~Hz}(1800$ lps; line scan rate 1 X; 256 * 256 pixels). Bars indicate $20 \mu \mathrm{m}$; drawing displays the position of the optical sections. Cytoplasm of the vSMCs (Acridin Red, orange) is clearly distinguishable on both sides of the lumen. Every optical section displays similar parts of the artery, indicative of stable image acquisition. Overall image quality suffers from high frame rates. Note the somewhat reduced quality of the images.

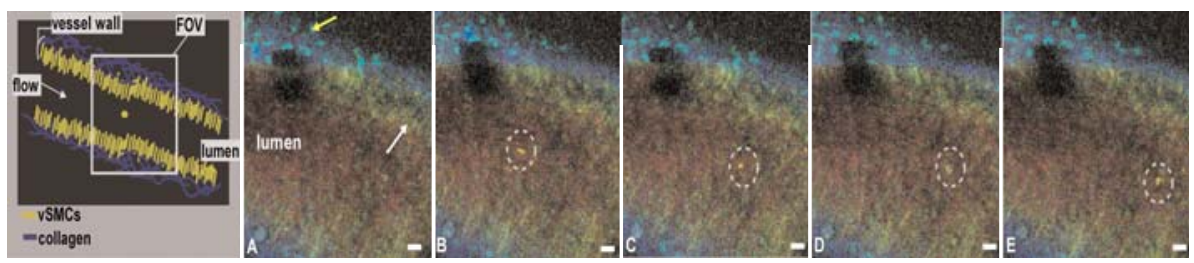

\section{Figure 4}

Five successive optical sections (A.E) of the left rinal artery of a WKY-rat in viro obtained by triggered acquisition at a frame rate of $5.9 \mathrm{~Hz}(1500 \mathrm{lps}$; line scan rate $1 \mathrm{X} ; 256$ * 256 pixels). Drawing displays the position of the optical sections; bars indicate $20 \mu \mathrm{m}$. Artery was labeled topically for cell nuclei (SYTO13, green) and systemically for cytoplasm of (blood) cells and platelets (acridin red, orange). The successive optical sections contain the same part of the artery. In the tunica adventitia, collagen (SHG, blue) and nuclei (yellow arrow) are visible; vSMC nuclei (white arrow) are observable in the tunica media. Moreover, an adhesive blood cell (encircled by white dotted line) is visible against the vessel wall in several (> 10) successive images. The lumen appears orange due to circulating unbound acridin red. 

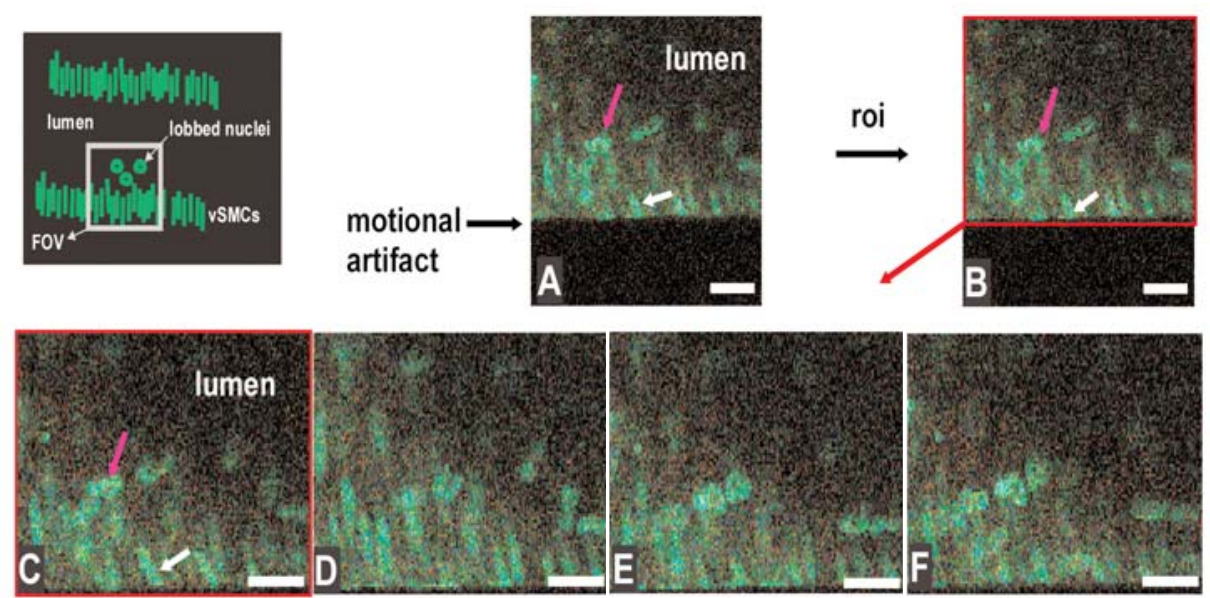

Figure 5

Optical section of the left carotid artery of a C57BL6/J mouse obtained in vivo by application of triggered image acquisition at a frame rate of $3.9 \mathrm{~Hz}(1000$ lps; line scan rate 1 X; 256 * 256 pixels). Cell nuclei were stained topically and systemically with SYTO13 (green). (A) Nuclei of vSMCs (white arrow) and several cells with lobbed nuclei (pink arrow) are distinguishable in the lumen. However, part of the optical section is disturbed by motion. At the bottom of the image, the artery has moved out of the focal plane. (B) This motional artifact can be removed by cropping the optical section. (C-F) Series of successive cropped images. The cells with lobbed nuclei (pink arrow) are slowly moving in the lumen. The optical sections were obtained at the end of the experiment, where the blood pressure of the mouse had dropped below $50 \mathrm{mmHg}$; heart rate was unaltered $(4.3 \mathrm{~Hz})$.

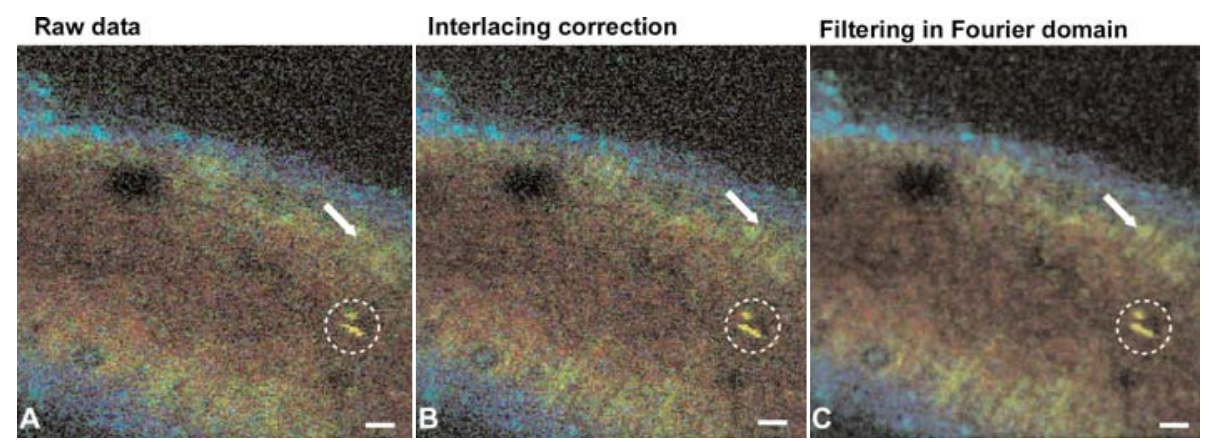

Figure 6

Effects of image analysis displayed on one of the optical sections of the series of images as shown in figure 4 (for extended information, see fig. 4). (A) Raw image of the data file as (partially) shown in fig. 4; image appears fuzzy. The vSMC nuclei (white arrow) are hardly recognizable as separate structures. (B) Same optical section after correction for inaccurate line positioning. The image already appears sharper; vSMC nuclei (white arrow) appear separated and a (slowly) moving rolling blood cell (encircled by white dotted line) is more clearly visible. Filtering in the Fourier domain (C) further improves S/N-ratio of the image. Nuclei of vSMCs (white arrow) are even more apparent. 


\section{References}

1. Megens, R.T., S. Reitsma, P.H. Schiffers, et al., Two-photon microscopy of vital murine elastic and muscular arteries. Combined structural and functional imaging with subcellular resolution. J Vasc Res, 2007;44(2):87-98.

2. Megens, R.T.A., M.G.A. oude Egbrink, J.P.M. Cleutjens, et al., Imaging collagen in intact and viable healthy and atherosclerotic arteries using fluorescently-labeled CNA35: towards molecular imaging of collagen in atherosclerotic plaques. Molecular Imaging, 2007;6(4):247-60.

3. Murphy, D.B., Fundamentals of light microscopy and electronic imaging. 1st edition ed. 2001, New York: Wiley-Liss Inc. 368.

4. van Gestel, M.A., S. Reitsma, D.W. Slaaf, et al., Both ADP and Thrombin Regulate Arteriolar Thrombus Stabilization and Embolization, but Are Not Involved in Initial Hemostasis as Induced by Micropuncture. Microcirculation, 2007;14(3):193-205.

5. oude Egbrink, M.G., G.H. Janssen, K. Ookawa, et al., Especially polymorphonuclear leukocytes, but also monomorphonuclear leukocytes, roll spontaneously in venules of intact rat skin: involvement of E-selectin. J Invest Dermatol, 2002;1 18(2):323-6.

6. Bosman, J., G.J. Tangelder, M.G. oude Egbrink, et al., Local application of adenosine induces an increase of capillary diameter in skeletal muscle of anesthetized rabbits. J Vasc Res, 1996;33(2): 111 -8.

7. Massberg, S., K. Brand, S. Gruner, et al., A critical role of platelet adhesion in the initiation of atherosclerotic lesion formation. J Exp Med, 2002;196(7):887-96.

8. Eriksson, E.E., J. Werr, Y. Guo, et al., Direct observations in vivo on the role of endothelial selectins and alpha(4) integrin in cytokine-induced leukocyte-endothelium interactions in the mouse aorta. Circ Res, 2000;86(5):526-33.

9. Eriksson, E.E., X. Xie, J. Werr, et al., Direct viewing of atherosclerosis in vivo: plaque invasion by leukocytes is initiated by the endothelial selectins. Faseb J, 2001;15(7):1149-57.

10. Pawley, J., Handbook of biological confocal microscopy. Third edition ed, ed. J. Pawley. 2006, New York: Springer. 985.

11. van Zandvoort, M., W. Engels, K. Douma, et al., Two-photon microscopy for imaging of the (atherosclerotic) vascular wall: a proof of concept study. J Vasc Res, 2004;41 (1):54-63.

12. Boulesteix, T., A.M. Pena, N. Pages, et al., Micrometer scale Ex Vivo multiphoton imaging of unstained arterial wall structure. Cytometry A, 2006;69(1):20-6.

13. Ferrara, D.E., D. Weiss, P.H. Carnell, et al., Quantitative 3D fluorescence technique for the analysis of en face preparations of arterial walls using quantum dot nanocrystals and twophoton excitation laser scanning microscopy. Am J Physiol Regul Integr Comp Physiol, 2006;290(1):R $114-23$

14. Maffia, P., B.H. Zinselmeyer, A. lalenti, et al., Images in cardiovascular medicine. Multiphoton microscopy for 3-dimensional imaging of lymphocyte recruitment into apolipoprotein-Edeficient mouse carotid artery. Circulation, 2007;1 15(1 1):e326-8. 
15. Svoboda, K., W. Denk, D. Kleinfeld, et al., In vivo dendritic calcium dynamics in neocortical pyramidal neurons. Nature, 1997;385(6612):161-5.

16. Ashworth, S.L. and G.A. Tanner, Fluorescent labeling of renal cells in vivo. Nephron Physiol, 2006; 103(2):p91-6.

17. Dunn, K.W. and P.A. Young, Principles of multiphoton microscopy. Nephron Exp Nephrol, 2006; 103(2):e33-40.

18. Dunn, K.W., R.M. Sandoval, K.J. Kelly, et al., Functional studies of the kidney of living animals using multicolor two-photon microscopy. Am J Physiol Cell Physiol, 2002;283(3):C905-16.

19. Padera, T.P., B.R. Stoll, P.T. So, et al., Conventional and high-speed intravital multiphoton laser scanning microscopy of microvasculature, lymphatics, and leukocyte-endothelial interactions. Mol Imaging, 2002;1(1):9-15.

20. Worbs, T., T.R. Mempel, J. Bolter, et al., CCR7 ligands stimulate the intranodal motility of $T$ lymphocytes in vivo. J Exp Med, 2007;204(3):489-95.

21. Campagnola, P.J., A.C. Millard, M. Terasaki, et al., Three-dimensional high-resolution secondharmonic generation imaging of endogenous structural proteins in biological tissues. Biophys J, 2002;82(1 Pt 1):493-508.

22. Zoumi, A., A. Yeh, and B.J. Tromberg, Imaging cells and extracellular matrix in vivo by using second-harmonic generation and two-photon excited fluorescence. Proc Natl Acad Sci U S A, 2002;99(17): $11014-9$.

23. Zoumi, A., X. Lu, G.S. Kassab, et al., Imaging coronary artery microstructure using secondharmonic and two-photon fluorescence microscopy. Biophys J, 2004;87(4):2778-86.

24. de Carvalho, H.F. and S.R. Taboga, Fluorescence and confocal laser scanning microscopy imaging of elastic fibers in hematoxylin-eosin stained sections. Histochem Cell Biol, 1996; 106(6):587-92.

25. Konig, K., Multiphoton microscopy in life sciences. J Microsc, 2000;200 ( Pł 2):83-104.

26. Janssen, B.J., T. De Celle, J.J. Debets, et al., Effects of anesthetics on systemic hemodynamics in mice. Am J Physiol Heart Circ Physiol, 2004;287(4):H1618-24.

27. Kurtz, R., M. Fricke, J. Kalb, et al., Application of multiline two-photon microscopy to functional in vivo imaging. J Neurosci Methods, 2006;151(2):276-86.

28. McConnel, G., Improving the penetration depth in multiphoton excitation laser scanning microscopy. Journal of Biomedical optics, 2006; 1 1(5):054020 1-7.

29. Zhao, X., Y.R. Chen, G. He, et al., Endothelial nitric oxide synthase (NOS3) knockout decreases NOS2 induction, limiting hyperoxygenation and conferring protection in the postischemic heart. Am J Physiol Heart Circ Physiol, 2007;292(3):H1541-50.

30. Prinzen, L., R.J. Miserus, A. Dirksen, et al., Optical and magnetic resonance imaging of cell death and platelet activation using annexin a5-functionalized quantum dots. Nano Lett, 2007;7(1):93-100. 
Chapter 7 


\section{Chapter 8}

\section{General Discussion}


Chapter 8 
The studies presented in this thesis demonstrate that Two-Photon Laser Scanning Microscopy (TPLSM) enables subcellular imaging of viable and intact large arteries of mice and rats in an ex vivo or in vivo environment.

Application of a model of mounted arteries combined with appropriate fluorescent probes, enabled visualization of various structural properties of both healthy and atherosclerotic arteries. Detailed insight was provided into differences in arterial vessel wall structure of elastic and muscular arteries. Furthermore, we assessed that the activation of the vascular endothelium in atherosclerosis-prone mice is not limited to the atherosclerosis prone sites. Moreover, we demonstrated the association between collagen and inflammation within atherosclerotic lesions. The feasibility of functional imaging was explored and two novel fluorescent probes, for collagen and for nitric oxide, were evaluated. Finally, we have shown the feasibility of stable in vivo imaging of large arteries by triggering at a fixed moment in the cardiac and respiratory cycle.

For ex vivo imaging, large arteries were mounted and fluorescently labeled in a perfusion chamber containing physiological buffer at $37^{\circ} \mathrm{C}$. Mounting of arteries is a preparation method that is generally used for perfusion myography and pharmacological evaluation of arteries [1,2]. As described in this thesis, this model has several advantages over existing preparation procedures like histology and en-face imaging. One of the main advantages is the possibility to mimic physiological conditions by maintaining physiological transmural pressure and length, resulting in structurally undisturbed arteries. For example, in chapter 3 it was shown that the elastic laminae appear straight without the wave-like appearance as seen in dissected arteries or histological slices. Removal of transmural pressure and length adjustment in our set-up resulted in appearance of wave-like elastin. This wave-like appearance is often interpreted as the normal morphology [3], but it appears to be an artifact of preparation. Another advantage is that the viability of all types of cells in the arterial vessel wall can be maintained for at least four hours (chapter 3). Indeed, we reported that, using a vasodilatation-vasoconstriction protocol, the functionality of endothelium and vascular smooth muscle cells in mounted arterial segments is preserved (chapter 3). In the future this allows the combination of imaging and functional studies (functional imaging). Perfusion of mounted arterial segments will enable visualization of structural consequences of flow dependent remodeling, blood cell-vessel wall interactions, thrombus formation, or leukocyte diapedesis. In chapter 4 we observed that the permeability of the arterial vessel wall is not influenced in this mounting model and that the endothelial cell lining remains intact, as derived from imaging patterns using a 
combination of nuclear (SYTO41) and collagen (CNA35/OG488) staining. Moreover, since no leukocytes (or platelets) adhered to the endothelium of healthy arteries after the dissection procedure (chapters 3 and 5), isolation of the arteries appears to have no immediate effect on the activation state of the vascular endothelium. Finally, the presence of the very delicate endothelial glycocalyx in mounted arteries is indicative of the undamaged condition of mounted large arteries, and proves that for physiological circumstances are being mimicked (chapter 3).

A crucial advantage of the application of TPLSM in the mounted geometry is the ability to explore the complete artery for detailed structural characteristics with high sensitivity. Applications are numerous, amongst others; further characterization of the role of the endothelial glycocalyx in atherosclerosis (thickness, distribution), the detection of angiogenesis in atherosclerotic plaques, and the visualization of the expression of growth factors, chemo- and cytokines as well as the presence of specific cell types (for example dendritic cells, progenitor cells) in (diseased) arteries. However, a prerequisite for this to be achieved is the existence of suitable probes for each of these imaging targets. When fulfilled, TPLSM imaging of mounted healthy and diseased arteries opens avenues for a wide range of studies on structural-functional relationships in the vessel wall. As a proof of concept for such studies, we have visualized the differences in morphology of elastic (carotid) and muscular (firstorder mesenteric and uterine) large arteries (chapter 3). Subtle dissimilarities, such as a higher number and larger diameter of fenestrae in the internal elastic lamina of muscular arteries, were assessed. Fenestrae in the internal elastic lamina have been associated with electrical coupling between vascular smooth muscle cells and endothelial cells [4-6] which indeed is more prominent in muscular than in elastic arteries [7]. Other dissimilarities detected between these arteries were a different vascular smooth muscle cell density (and, hence, a different size), a third intermediate elastic lamina in elastic arteries, and the morphology of collagen in the tunica adventitia (using second harmonic generation).

Collagen in the other tunicae of the arterial vessel wall could not be visualized using its second harmonic generation. Therefore, CNA35 labeled with fluorescent Oregon Green (CNA35/OG488) [8] was implemented as a collagen marker for (at least) type I, III, and IV collagen in healthy and diseased large arteries (chapter 4, 5).

Both after ex vivo and in vivo administration, we detected that in healthy and viable elastic arteries this probe was only able to highlight collagenic structures 
in the adventitia, due to limited penetration of the probe through the intact endothelial cell barrier and the elastic laminae. In contrast, in arteries of atherosclerosis-prone (hyperlipidemic) ApoE $\%$ mice fed a western diet, CNA35/OG488 labeled the collagen network inside atherosclerotic lesions, both in the plaque and in the shoulder region. Furthermore, the collagen in the basal membrane of the tunica intima was labeled at both plaque and nonplaque sites. This indicates the barrier function of the endothelial cell lining to be hampered, probably as a consequence of endothelial activation. The latter was confirmed by the presence of inflammatory cells (probably monocytes) adhering to the endothelium, mainly at sites of atherosclerotic plaques but also at sites that are not prone to atherosclerosis. These findings suggest that in atherosclerosis endothelial activation is generalized and occurs throughout the larger vessels. The capability of CNA35/OG488 to label plaques in vivo suggests that CNA35/OG488 is a good molecular imaging agent for atherosclerosis. A requirement for (clinical) molecular imaging is the application of non-invasive techniques, such as magnetic resonance imaging (MRI) or positron emission tomography (PET). To investigate whether CNA35 can be used with these techniques, we are currently characterizing CNA35 conjugated to strongly fluorescent quantum dots (QDs). The use of $Q D$ s as a vehicle for molecular imaging was recently demonstrated in a study where we developed a multimodal optical-MRI probe for apoptosis detection [9]. Annexin V was conjugated to QDs to detect apoptosis optically, while MRI detection was enabled by loading the QD with Gadolinium wedges. The strength of the combination of TPLSM and MRI is evident. While MRI is non-invasive, it lacks resolution and sensitivity; this gap can be bridged by combining it with optical techniques such as TPLSM. Interestingly, CNA35/OG488 labeling may also be a measure of plaque stability, since collagen in atherosclerotic lesions is a major determinant for this. However, since plaque stability is undoubtedly a plural process, unequivocal detection of vulnerable plaques will require multitargeted probes.

Beside an increase in cell permeability [10], atherosclerotic endothelial cells are also characterized by altered nitric oxide (NO) production [11]. NO is important for many functional processes in the arterial vessel wall such as (endothelium-derived) vasodilatation [12, 13] and anti-inflammation [11]. Decreased NO-production due to endothelial dysfunction is linked to various diseases such as atherosclerosis [13-15]. However, up to now, NO production and NO signaling were mainly studied indirectly, due to a lack of suitable NOprobes. In our hands, the currently available NO-probes such as Diaminofluoresceins (DAF) [16], appeared not to be suitable for NO-imaging 
due to non-specificity and photo-activation, as has also been described in literature $[17,18]$. In chapter 6 , we evaluated a member of the family of Fluorescent Nitric Oxide Chelotropic Traps (FNOCT-5/FNOCT-4 [19]) in an in vitro model for angiogenesis. In these cultured endothelial cells, the highly selective FNOCT was used for the detection of NO production in sprouting but not in (neighboring) non-sprouting cells, demonstrating that FNOCT allows localized detection of NO. At present, further characterization of the properties of FNOCT is ongoing by investigating its potential for penetration into intact, viable mounted arteries (and other tissues) ex vivo and its response to stimuli of vasodilatation and vasoconstriction. This might enable translation of results obtained in vitro to intact large arteries. Thus, application of FNOCT in mounted arteries holds promise for future visualization of $\mathrm{NO}$ production in intact large arteries, again an avenue for functional imaging as described before.

Although the present study showed that TPLSM clearly enhances the possibilities to study e.g. structural and functional changes in the arterial wall during atherogenesis (chapter 5), limitations of this technique became evident as well. Due to the accumulation of fat in the necrotic core, we were not able to visualize the advanced plaque at its full depth. Enhancing penetration depth of TPLSM by narrowing the pulse width of the excitation beam from 140 femtoseconds (fs) to around $30 \mathrm{fs}$ will increase the possibility of two-photon excitation deeper in the plaque. It is reported that this may result in increases of penetration depth by a factor 2 to 4 [20]. We are currently establishing this change in our TPLSM set-up.

In vivo application of (untriggered) TPLSM imaging to especially the larger arteries (chapter 7) is severely hampered by motional disturbances due to heartbeat and respiration. Most images from a series of subsequent images were of poor quality due to movement of the sample and consequent loss of focus. Increasing acquisition rates led to series of comparable images with relatively limited motional disturbances. However, most images were still of poor quality and contained different parts of the sample. Moreover, image quality is reduced by increased acquisition rates, due to a reduced signal-tonoise ratio, although image analysis may be used to improve this. Application of a new imaging mode that uses triggered acquisition of each image on the cardiac and respiratory cycle resulted in series of subsequent images that all display the same part of the artery during the same phase of movement. When this triggered image acquisition was performed with increased acquisition rates, motional disturbances within the image could be reduced even further. 
Optimal timing of the trigger pulse was determined empirically and proved to be at the start of the diastolic phase of the cardiac cycle. The presented method holds potential for the visualization of the relationship between various structural and functional properties in healthy and diseased arteries in vivo. Although we have shown the feasibility of in vivo imaging of large arteries using TPLSM, application still has some important limitations. Accurate tracking of (slowly) moving cells or quantification of functional properties of the vessel wall such as NO production is virtually impossible due to variable and relatively long time intervals between two subsequent images. This problem can only be circumvented by applying the triggering method in higher speed twophoton microscopy. Interesting developments are currently ongoing, amongst others the incorporation of resonant scanning mirrors and multiple laser beams $[21,22]$. Moreover, the shortening of laser pulse width, as referred to previously [20], might be advantageous for in vivo imaging since the probability for two-photon excitation results in an increase of the signal-to-noise ratio.

Future research with TPLSM on alterations that occur during atherosclerosis or other vascular diseases may lead to better understanding of the disease processes and thereby to better diagnosis and treatment.

\section{References}

1. Bergaya, S., R.H. Hilgers, P. Meneton, et al., Flow-dependent dilation mediated by endogenous kinins requires angiotensin AT2 receptors. Circ Res, 2004;94(12): 1623-9.

2. Hilgers, R.H., S. Bergaya, P.M. Schiffers, et al., Uterine Artery Structural and Functional Changes During Pregnancy in Tissue Kallikrein-Deficient Mice. Arterioscler Thromb Vasc Biol, 2003;23(10): 1826-1832.

3. Boulesteix, T., A.M. Pena, N. Pages, et al., Micrometer scale Ex Vivo multiphoton imaging of unstained arterial wall structure. Cytometry A, 2006;69(1):20-6.

4. Dora, K.A., S.L. Sandow, N.T. Gallagher, et al., Myoendothelial Gap Junctions May Provide the Pathway for EDHF in Mouse Mesenteric Artery. J Vasc Res, 2003;40(5):480-490.

5. Sandow, S.L., K. Goto, N.M. Rummery, et al., Developmental changes in myoendothelial gap junction mediated vasodilator activity in the rat saphenous artery. J Physiol, 2004;556 (Pt 3):875-86.

6. Sandow, S.L., M. Tare, H.A. Coleman, et al., Involvement of myoendothelial gap junctions in the actions of endothelium-derived hyperpolarizing factor. Circ Res, 2002;90(10):1 108-13.

7. Hill, C.E., J.K. Phillips, and S.L. Sandow, Heterogeneous control of blood flow amongst different vascular beds. Med Res Rev, 2001;21(1):1-60.

8. Krahn, K.N., C.V. Bouten, S. van Tuijl, et al., Fluorescently labeled collagen binding proteins allow specific visualization of collagen in tissues and live cell culture. Anal Biochem, 
2006;350(2):177-85.

9. Prinzen, L., R.J. Miserus, A. Dirksen, et al., Optical and magnetic resonance imaging of cell death and platelet activation using annexin a5-functionalized quantum dots. Nano Lett, 2007;7(1):93-100.

10. Gimbrone, M.A., Jr., Vascular endothelium, hemodynamic forces, and atherogenesis. Am J Pathol, 1999;155(1):1-5.

11. Loscalzo, J. and J.A. Vita, Nitric Oxide and the Cardiovascular System. Contempory Cardiology, ed. C.P. Cannon. 2000, Totowa, New Jersey: Humana Press. 601.

12. Fleming, I. and R. Busse, Signal transduction of eNOS activation. Cardiovasc Res, 1999;43(3):532-41.

13. Shesely, E.G., N. Maeda, H.S. Kim, et al., Elevated blood pressures in mice lacking endothelial nitric oxide synthase. Proc Natl Acad Sci U S A, 1996;93(23):13176-81.

14. Dam, B.v., Nitric oxide and oxidative stress; focus on endthelial activation and diabetesinduced arteriolar changes, in Faculteit der Geneeskunde. 2003, Vrije Universiteit Amsterdam: Amsterdam. 158.

15. Ignarro, L.J., R.E. Byrns, G.M. Buga, et al., Endothelium-derived relaxing factor from pulmonary artery and vein possesses pharmacologic and chemical properties identical to those of nitric oxide radical. Circ Res, 1987;61 (6):866-79.

16. Kojima, H., Y. Urano, K. Kikuchi, et al., Fluorescent Indicators for Imaging Nitric Oxide Production. Angew Chem Int Ed Engl, 1999;38(21):3209-3212.

17. Broillet, M., O. Randin, and J. Chatton, Photoactivation and calcium sensitivity of the fluorescent NO indicator 4,5-diaminofluorescein (DAF-2): implications for cellular NO imaging. FEBS Lett, 2001;491(3):227-32.

18. Zhang, X., W.S. Kim, N. Hatcher, et al., Interfering with nitric oxide measurements. 4,5diaminofluorescein reacts with dehydroascorbic acid and ascorbic acid. J Biol Chem, 2002;277(50):48472-8.

19. Meineke, P., U. Raven, H. de Groot, et al., Nitric oxide detection and visualization in biological systems. Applications of the FNOCT method. Biol Chem, 2000;381 (7):575-82.

20. McConnel, G., Improving the penetration depth in multiphoton excitation laser scanning microscopy. Journal of Biomedical optics, 2006; 1 1(5):054020 1-7.

21. Kurtz, R., M. Fricke, J. Kalb, et al., Application of multiline two-photon microscopy to functional in vivo imaging. J Neurosci Methods, 2006;151(2):276-86.

22. Padera, T.P., B.R. Stoll, P.T. So, et al., Conventional and high-speed intravital multiphoton laser scanning microscopy of microvasculature, lymphatics, and leukocyte-endothelial interactions. Mol Imaging, 2002;1(1):9-15. 


\section{Chapter 9}

Summary

Samenvatting

Dankwoord

Curriculum Vitae

List of Publications 
Chapter 9 


\section{Summary}

The circulation is a complex system consisting of different types of blood vessels. It is now recognized that these vessels are part of a subtle regulatory system with differential properties along the vascular tree. Alterations in the vessel wall may lead to various diseases such as atherosclerosis. Current knowledge of such vascular alterations is mostly based on histological studies of isolated samples that have lost viability. Functional properties of various compounds in the atherosclerotic vessel wall are still largely unknown. Better understanding of the functionality of the atherosclerotic arterial wall, and, thus, increased insight in (development of) atherosclerotic lesions requires studying of these properties in vivo or in viable arteries ex vivo. This thesis focuses on the vessel wall of large murine arteries and cultured cell systems using twophoton laser scanning microscopy (TPLSM) as an imaging tool for studying alterations in vessel wall properties during atherogenesis and their functional consequences.

In chapter 2, a brief literature survey is given of the biology of large arteries, the process of atherosclerosis, and animal models that can be used to study atherosclerosis. Then, the basic principles of conventional (single-photon) microscopy and TPLSM are discussed and compared. In addition, a short overview is dedicated to the fluorescent labeling of vascular structures and the current status of the applications of TPLSM for visualization of various aspects of larger arteries is discussed.

In chapter 3 we evaluated applicability of TPLSM in large elastic and small muscular arteries under physiological conditions. To this purpose we developed a perfusion chamber model in which the arteries were mounted. Their viability was assessed and structural components such as elastin, collagen, nuclei, and endothelial glycocalyx were visualized. Moreover, functionality of the arteries was determined using diameter changes in response to noradrenaline and acetylcholine. We conclude that TPLSM enables visualization and quantification of subcellular structures in vital and functional elastic and muscular murine arteries, allowing unraveling of structure-function relationships in healthy and diseased arteries.

Atherogenesis involves structural and cellular changes in the vessel wall of large arteries. In advanced atherosclerosis, collagen content is considered to be a major determinant for plaque stability. In chapter 4 , the novel fluorescent 
collagen marker CNA35/OG488 was used to assess the distribution of collagen in mounted viable large arteries of control (C57BL6/J) and atherosclerotic (ApoE $\%$ ) mice. The labeling characteristics of CNA35/OG488 were evaluated and compared with histology. Furthermore, the mounting method was adapted to visualize atherosclerotic lesions in the carotid artery bifurcation. The luminal uptake of CNA35/OG488 in mounted healthy arteries appeared to be limited by an intact endothelial layer and elastic laminae. However, in mounted atherosclerotic arteries, CNA35/OG488 did penetrate into the vessel wall and labeled the collagen structure in atherosclerotic lesions, indicative of alterations in endothelium and elastic laminae. Intravenous administration of CNA35/OG488 in living mice revealed that CNA35/OG488 might have potential as a molecular imaging marker for atherogenesis.

In chapter 5 we visualized early and advanced atherosclerotic lesions in the carotid artery bifurcation as well as the direct relationship between collagen and inflammatory cells in intact viable mounted carotid arteries of control and atherosclerotic mice, at subcellular level. Isolated carotid arteries of ApoE $\%$ mice, aged 15 or 21 weeks, after 7 and 13 weeks on a western diet, and of C57BL6/J control mice fed a chow diet were mounted in a perfusion chamber, pressurized, and labeled with specific fluorescent markers for cell nuclei, inflammatory cells, collagen, and lipids. Special attention was paid to the fibrous cap regions and the various vessel wall layers. Control vessels had intact endothelium without adhering blood cells or significant intimal collagen labeling. In contrast, in $\mathrm{ApoE}^{\%}$ mice already at 15 weeks inflammatory cells adhered to the endothelium, while at the same time increased labeling of collagen was observed in the tunica intima both at lesion-prone and non-lesionprone sites, both indicating endothelial activation. In plaques, located exclusively in carotid bifurcations of $\mathrm{ApoE}^{\%}$ mice, internalized inflammatory cell density increased with age and plaque progression. Interestingly, this was observed both in the tunica adventitia and in the tunica intima, while in the tunica media no inflammatory cells were detected at both ages. In the whole plaque, aging or plaque progression did not alter the direct relation between inflammatory cells and collagen. However, within the fibrous caps specifically, direct contact between inflammatory cells and collagen increased with age. Altogether, this study indicated that visualization of viable and structurally intact arteries provides new and detailed insight in the complex relationship between inflammatory cells and collagen in fibrous caps and whole plaques during atherogenesis and suggests involvement of the tunica adventitia in atherogenesis. 
To further illustrate the applicability of TPLSM to functional studies on blood vessels, we studied nitric oxide (NO) production. Up till now most knowledge concerning the role of $\mathrm{NO}$ in arteries is based on indirect measurements. The importance of $\mathrm{NO}$ was never directly established in viable arteries by visualizing $\mathrm{NO}$ production in the vessel wall. To this end, a fluorescent probe is needed which relates changes in the presence of $\mathrm{NO}$ with changes in fluorescent signal. Furthermore, since $\mathrm{NO}$ exist for only very short periods of time such probe must be highly sensitive. In chapter 6 we characterized and applied a Fluorescent Nitric-oxide Cheletropic Trap (FNOCT) for sensitive NO detection using TPLSM. Under physiological conditions the membranepermeable ester FNOCT-5 neither significantly inhibits enzymatic NO production, nor acts as superoxide scavenger. We applied FNOCT-5 to sprouting and non-sprouting HUVECs cultured on beads in fibrin matrix. Intracellular enzymes transform FNOCT-5 into its accumulating dicarboxylate FNOCT-4. On binding NO, FNOCT-4 fluorescence undergoes a red to blue shift. We used the ratio of blue to red fluorescence as measure of $\mathrm{NO}$ production. Sprouting and non-sprouting cells that were not additionally stimulated did not produce detectable amounts of NO. However, in the same cell culture, sprouting cells showed significantly higher $\mathrm{NO}$ production than their non-sprouting counterparts when stimulated by calcium ionophore. Stimulation with acetylcholine resulted in even larger differences. These data show that visualization of cheletropic traps allows detection of local variations in NO production with subcellular resolution in physiologically relevant three dimensional systems and holds potential for application in viable arteries.

In vivo imaging of especially large arteries using TPLSM results in images (optical sections) that severely suffer from motional artifacts within each image and between subsequent images. In chapter 7 we applied TPLSM image acquisition in vivo, triggered on cardiac and respiratory cycle; in addition image acquisition was accelerated in order to reduce the impact of motion between subsequent images and within each image. Carotid arteries of anesthetized C57BL6/J mice and renal arteries of anesthetized WKY-rats were surgically exposed, labeled with specific fluorescent markers for cell nuclei and cytoplasm, and imaged using TPLSM. Blood pressure and respiratory signals were recorded as triggering signals for (accelerated) image acquisition. Filtering in the Fourier domain was explored to improve overall image quality. In both mice and rats, timing of the trigger moment at the diastolic phase in the cardiac cycle combined with acquisition rates of $4 \mathrm{~Hz}$ or higher, resulted in stable images with reduced motional artifacts. Moreover, subsequently acquired images contained a similar and stable image of the artery. 
Application of the Fourier transformation further improved overall image quality.

We have shown the feasibility of stable imaging of large arteries in vivo using TPLSM triggered on cardiac and respiratory cycle. The presented method creates new opportunities for in vivo studying of various structural and functional properties of the (diseased or damaged) arterial wall at subcellular level.

As described in the general discussion (chapter 8), the studies presented in this thesis demonstrate that TPLSM enables subcellular imaging of viable and intact large arteries of mice and rats in an ex vivo or in vivo environment. Application of our model of mounted arteries combined with appropriate fluorescent probes enables visualization of various structural properties of both healthy and atherosclerotic arteries. Detailed insight was provided into differences in vessel wall structure of elastic and muscular arteries. Furthermore, we assessed that the activation of the vascular endothelium in atherosclerosis-prone mice is not limited to the atherosclerosis prone sites and we demonstrated the association between collagen and inflammation within atherosclerotic lesions. The feasibility of functional imaging was explored and two novel fluorescent probes, for collagen and for nitric oxide, were evaluated. Finally, we have shown the feasibility of stable in vivo imaging of large arteries by triggering at a fixed moment in the cardiac and respiratory cycle.

We conclude that the described techniques offer a new and different view on healthy and diseased arteries which provides new insight in various structural and functional properties of atherogenesis. Further development of these techniques holds potential for future applications in both a scientific and clinical environment. 


\section{Samenvatting}

De circulatie van bloed in het menselijk lichaam gaat door een complex systeem dat bestaat uit arteriële (aanvoerende) en veneuze (afvoerende) bloedvaten, met daartussenin de microcirculatie met de capillairen (haarvaten). De vaatwandstructuur en ook de functies van de diverse typen bloedvaten zijn verschillend. Veranderingen in eigenschappen van de arteriële vaatwand kunnen leiden tot ziekten zoals atherosclerose (aderverkalking). Atherosclerose is een systemische ziekte van de grote arteriën en kan ernstige klinische complicaties tot gevolg hebben zoals hartfalen en herseninfarcten. Atherosclerose is de onderliggende oorzaak voor $50 \%$ van alle sterftegevallen in de westerse wereld.

De huidige kennis over de veranderingen in de arteriële vaatwand tijdens atherosclerose is voornamelijk gebaseerd op histologische studies die zijn vitgevoerd in geïsoleerd, niet vitaal weefsel. Ondanks de grote bijdrage van histologische studies aan onze huidige kennis over bloedvaten, zijn de mogelijkheden tot het bestuderen van de functionele eigenschappen van structuren in de vaatwand zeer beperkt. Een verbeterd inzicht in de combinatie van structurele en functionele eigenschappen van de vaatwand kan mogelijk leiden tot meer kennis over (de ontwikkeling van) atherosclerotische laesies. Hiertoe dient gebruik gemaakt te worden van intacte en vitale bloedvaten, waarvan de vaatwand bestudeerd moet worden met nieuwe optische technieken met voldoende penetratiediepte. Een voorbeeld van zo'n techniek is fluorescentie microscopie gebaseerd op twee-foton excitatie.

Dit proefschrift is gericht op het toepassen van twee-foton laser scanning microscopie (TPLSM) voor het visualiseren van zowel structurele als functionele veranderingen in de vaatwand ten gevolge van atherosclerose.

Hoofstuk 2 betreft een literatuur overzicht waarin de biologie van de grotere arteriën en de ontwikkeling van atherosclerose wordt beschreven. Er wordt ingegaan op de verschillende diermodellen om atherosclerose te bestuderen, en daarnaast worden de basisbeginselen van conventionele en twee-foton fluorescentie microscopie beschreven. Ook worden diverse fluorescente markers die geschikt zijn voor het aankleuren van vaatwandstructuren in intacte en vitale bloedvaten gepresenteerd. Tenslotte is een overzicht gegeven van recent gepubliceerde toepassingen van twee-foton microscopie voor visualisatie van bloedvaten.

In hoofdstuk 3 wordt de toepasbaarheid van twee-foton microscopie voor het visualiseren van vitale intacte elastische en musculaire arteriën van muizen 
beschreven. Om dit mogelijk te maken is een perfusiekamer ontwikkeld waarin de vitaliteit en structuur van deze bloedvaten wordt bestudeerd. Componenten van de vaatwand van de in de perfusiekamer opgespannen arteriën, zoals elastine, diverse soorten cellen en de endotheliale glycocalyx, zijn gevisualiseerd. Bovendien is de functionaliteit van de opgespannen arteriën geëvalueerd door bepaling van diameter veranderingen ten gevolge van noradrenaline en acetylcholine stimulatie. Uit de resultaten blijkt dat TPLSM in combinatie met het perfusiekamer model het mogelijk maakt om structurele en functionele verschillen tussen diverse typen bloedvaten te bestuderen en zodoende een bijdrage kan leveren aan de kennis over de vaatwand en diverse (vasculaire) ziekten.

De veranderingen in de vaatwand ten gevolge van atherosclerose hebben betrekking op zowel cellulaire als structurele vaatwandcomponenten. In vergevorderde stadia van atherosclerotische laesies wordt de aanwezigheid van collageen en zijn structuur gezien als een belangrijke determinant voor de stabiliteit van deze laesies. In hoofdstuk 4 is de distributie van collageen in opgespannen intacte en vitale arteriën van muizen bestudeerd door gebruik te maken van een nieuw specifiek label voor collageen, genaamd CNA35/OG488. Voor deze studie is tevens het perfusiekamer model, zoals beschreven in hoofdstuk 3, aangepast zodat ook de bifurcatie (splitsing) van de arteria carotis (halsslagader) kan worden opgespannen en gevisualiseerd. De labelingskarakteristieken van CNA35/OG488 in vitale gezonde en atherosclerotische arteriën zijn geëvalueerd en vergeleken met histologische gegevens.

De resultaten laten zien dat de opname van CNA35/OG488 in de gezonde vaatwand zeer beperkt is. Echter, in atherosclerotische carotiden blijkt CNA35/OG488 wel in de vaatwand door te dringen en collageen structuren in atherosclerotische laesies te labelen. Intraveneuze toediening van CNA35/OG488 in levende muizen laat verder zien dat CNA35/OG488 mogelijk potentie heeft als een moleculaire marker voor detectie van atherosclerotische laesies in vivo.

In de studie beschreven in hoofdstuk 5 zijn de atherosclerotische laesies in de bifurcatie van de carotiden van atherosclerotische muizen (met een leeftiid van 15 en 21 weken) gevisualiseerd tot op subcellulair niveau $(<1 \mu \mathrm{m})$. De ontwikkeling van atherosclerotische laesies is gevisualiseerd vanaf het initiële stadium tot aan de vergevorderde stadia. Daarnaast hebben we de relatie tussen collageen structuren en ontstekingscellen (inflammatoire cellen) in drie stadia van atherosclerose bestudeerd en gekwantificeerd. 
In gezonde (controle) arteriën zijn geen aan het endotheel hechtende bloedcellen waargenomen. Ook is er geen significante collageen kleuring zichtbaar in de vaatwand. Dit is in tegenstelling tot arteriën van atherosclerotische muizen. Al in atherosclerotische muizen van 15 weken oud zijn er aan de endotheellaag hechtende bloedcellen zichtbaar. Ook is de hoeveelheid collageen kleuring in de intimale en mediale vaatwandlagen verhoogd, zowel op plaque-gevoelige locaties (bifurcatie) als op plaqueongevoelige locaties in de arteriën. Dit is een aanwijzing voor het feit dat in deze muizen de arteriële endotheelcellen geactiveerd zijn.

In de atherosclerotische laesies, die alleen in de bifurcatie van de carotiden waargenomen zijn, neemt de hoeveelheid geïnternaliseerde/geïnfiltreerde inflammatoire cellen toe met leeftiid en plaque progressie. Deze toename is gevonden in de binnenste (tunica intima) en de buitenste (tunica adventitia) laag van vaatwand. In de middelste laag (tunica media) zijn geen inflammatoire cellen waargenomen. Wanneer een atherosclerotische laesie als één geheel wordt beschouwd zijn er geen effecten van leeftiij en plaque progressie gevonden op de relatie tussen collageen en inflammatoire cellen. In de fibreuze kappen van plaques, echter, blijkt het aantal inflammatoire cellen in direct contact met collageen wel toe te nemen met leeftijd.

Deze studie toont aan dat visualisatie van vitale en structureel intacte arteriën nieuwe en gedetailleerde inzichten kan verschaffen in de complexe relatie tussen inflammatoire cellen en collageen tijdens de ontwikkeling van atherosclerose (atherogenese). Bovendien laten de resultaten zien dat ook veranderingen optreden in de tunica adventitia tijdens de ontwikkeling en progressie van atherosclerotische plaques.

Ter illustratie van de bruikbaarheid van TPLSM om functionele eigenschappen van bloedvaten te bestuderen, is endotheliale stikstof oxide (NO) productie bestudeerd. De meeste kennis met betrekking tot de rol van NO in grote bloedvaten is gebaseerd op indirecte metingen en niet op directe waarnemingen in nog intacte en levende bloedvaten. Om dit mogelijk te maken is een functionele fluorescerende marker nodig die een verandering in de aanwezigheid van NO koppelt aan een verandering in fluorescent signaal. Bovendien moet een NO marker zeer gevoelig zijn omdat die slechts voor zeer korte periodes in het weefsel aanwezig is.

In hoofdstuk 6 hebben wij als potentiële NO-marker het "fluorescent nitric oxide chelotropic trap" oftewel FNOCT gekarakteriseerd en toegepast voor NO-detectie met behulp van TPLSM. De membraan-permeabele ester FNOCT5 blijkt onder fysiologische condities niet op superoxides te reageren en de NO-productie niet te beïnvloeden. 
De bruikbaarheid van FNOCT-5 werd getest in een in vitro setup met gekweekte sproutende en niet-sproutende endotheelcellen. FNOCT-5 wordt door intracellulaire enzymen omgevormd tot de NO-sensitieve en sterk accumulerende sub-vorm dicarboxylate FNOCT-4, die onder invloed van NObinding een kleurverschuiving van het emissielicht ondergaat (van rode naar blauwe golflengte). Met behulp van FNOCT-5 is het verschil in NO-productie tussen gestimuleerde (acetylcholine en calcium-ionofoor) sproutende en nietsproutende endotheelcellen in beeld gebracht. Deze resultaten tonen aan dat FNOCT in combinatie met TPLSM het mogelijk maakt om lokale verschillen in NO-productie te visualiseren onder fysiologisch relevante omstandigheden en in 3 dimensies. Bovendien tonen de resultaten de potentie voor NO-detectie in vitale en structureel intacte bloedvaten aan.

Het afbeelden van met name de grotere bloedvaten in levende proefdieren (in vivo microscopie) leidt tot optische afbeeldingen die sterk lijden onder bewegingsartefacten. Deze bewegingsartefacten zijn waar te nemen in zowel de optische afbeeldingen zelf als tussen de achtereenvolgende optische afbeeldingen behorend bij een (tijd)serie van opnames. In hoofdstuk 7 is TPLSM in combinatie met getriggerde beeldacquisitie toegepast om deze in vivo bewegingsartefacten te verminderen. $\mathrm{Bij}$ getriggerde beeldacquisitie wordt het startmoment van beeldacquisitie bepaald door een extern start signaal, in dit geval een combinatie van bloeddruk- en ademhalingsignalen die zijn afgeleid van het proefdier. Naast getriggerde beeldacquisitie is tevens de acquisitiesnelheid verhoogd. Deze methode is toegepast voor het bestuderen van vrijgeprepareerde carotiden van C57BL6/J muizen en WKY ratten. Fluorescente kleuringen zijn gebruikt voor het specifiek aankleuren van cytoplasma en celkernen. Na acquisitie is filtering van de opnames in het Fourier domein toegepast om de algehele beeldkwaliteit verder te verbeteren. In zowel muizen als ratten blijkt dat beeldacquisitie, getriggerd op de diastole hartfase van de hartcyclus en gecombineerd met een acquisitiesnelheid van $4 \mathrm{~Hz}$ of meer, resulteert in stabiele opnames waarin bewegingsartefacten sterk gereduceerd zijn. Bovendien blijkt dat achtereenvolgende opnames binnen een tijdserie van opnames een vergelijkbaar deel van de halsslagader bevatten. Toepassing van Fourier transformatie leidde tot een verdere verbetering van de beeldkwaliteit.

Deze resultaten tonen aan dat het haalbaar is om grote arteriën in vivo te visualiseren op een subcellulair niveau door toepassing van TPLSM door middel van beeldacquisitie getriggerd op bloeddruk en ademhaling. Deze methode creëert nieuwe mogelijkheden voor het in vivo afbeelden van functionele en structurele eigenschappen van (zieke of beschadigde) grote bloedvaten. 
Zoals beschreven in de Algehele Discussie in hoofdstuk 8, demonstreren de in dit proefschrift beschreven studies dat twee-foton laser scanning microscopie (TPLSM) een krachtige techniek is om intacte grote bloedvaten van muizen en ratten te visualiseren op een subcellulair niveau. Toepassing van het perfusiekamer model in combinatie met specifieke fluorescerende kleurstoffen maakt het mogelijk diverse structurele eigenschappen van zowel gezonde als atherosclerotische bloedvaten af te beelden. Een gedetailleerd inzicht is gegeven in verschillen in vaatwandstructuur tussen elastische en musculaire arteriën. Uit de studies in atherosclerotische arteriën is gebleken dat activatie van het vasculaire endotheel niet is beperkt tot plekken waar atherosclerotische laesies veel voorkomen (met name bifurcaties). Bovendien hebben we de locatie van collageen in relatie tot de aanwezigheid van ontstekingscellen bepaald in atherosclerotische laesies. De haalbaarheid van het afbeelden van functionele eigenschappen van de vaatwand is aangetoond en twee nieuwe fluorescente kleuringen, voor collageen en $\mathrm{NO}$, zijn toegepast en geëvalueerd. Ten slotte is de haalbaarheid van het in vivo afbeelden van de vaatwand van grote arteriën aangetoond door middel van beeldacquisitie getriggerd op een vast moment in de hart- en ademhalingscyclus.

Hiervit kan worden geconcludeerd dat de beschreven technieken en methodes nieuwe mogelijkheden creëren om (gezonde en zieke) bloedvaten te bestuderen en nieuwe inzichten kunnen verschaffen in de structurele en functionele eigenschappen van ziektebeelden zoals atherosclerose. De verdere ontwikkeling van deze technieken kan mogelijk leiden tot nieuwe toepassingsgebieden voor zowel experimenteel als klinisch onderzoek. 
Chapter 9 


\section{Dankwoord}

Een welverdiende dank jullie wel voor alle (oud) collegae, coauteurs, leden van de beoordelingscommissie, familie en vrienden die de afgelopen jaren hebben bijgedragen aan dit proefschrift. Enkele mensen uit deze grote groep wil ik speciaal noemen voor hun onmisbare bijdrage:

Dick, Marc en Mirjam. Ik had me geen beter promotieteam kunnen wensen. Jullie vulden elkaar perfect aan en ik heb erg veel van jullie geleerd, zowel op het gebied van onderzoek als op persoonlijk vlak. Zelfs naast mijn promotietraject zijn jullie altijd nauw betrokken geweest en altijd beschikbaar gebleven voor hulp en gezelligheid. Dank voor al jullie inzet en ook voor de kans die jullie me gegeven hebben om dit promotietraject in te gaan.

(Oud) collegae van de voormalige vakgroep Biofysica: bedankt voor de vriendschap, alle gezelligheid tijdens en naast het werk (Thembi, Reitz, fietsen, film, muziek, etc.), het zetten van koffie, het meebrengen van lekkernijen en bovenal het creëren van een heel goede werksfeer.

Wim, de "vliegende keeper" van het tweefoton team en van mijn promotie traject. Dank voor al je razendsnelle hulp en vriendschap. Kim, ik vond het altijd erg fijn dat we tijdens de afgelopen 5 jaar bijna synchrone werktijden hebben gehad en ben blij dat je mijn paranimf wilt zijn. Lenneke, alle persoonlijke gesprekken en je kritische kijk op onderzoek waardeer ik enorm. Sietze, bedankt voor de discussies over zowel levensbeschouwelijke als vaktechnische onderwerpen. Joost, NERD en paranimf, dankjewel dat je aio bent geworden.

Ook een speciaal woord van dank voor (oud) collegae van andere vakgroepen en in het bijzonder: Jo, Paul, Fazzi, Rob, Marcus, Peter en Ben van Farmacologie; Viviane van Fysiologie; Imke, Johan, Judith, Marijke, Karen en Marion van Biochemie; Jack en Esther van Pathologie (AZM); Abdel van Cardiologie (AZM); Maarten Merkx, Carlijn Bouten, Ralf Boerboom, Katy Krahn en Ingrid Kieft van Biomedische technologie (TuE).

De BMT (afstudeer) stagiaires Han, Ellen, Leoni, Harald, en Richard wil ik bedanken voor hun inzet, bijdrage en hun vaak verfrissende kijk op het onderzoek. Het onderzoeksinstituut CARIM wil ik bedanken voor het creëren van een leerzaam promotietraject. Daarnaast wil ik alle CARIM promovendi van 2002-2007 bedanken voor de gezelligheid en saamhorigheid gedurende de vele cursussen. Inge en Hans, hartelijk dank voor de mooie opmaak van zowel mijn proefschrift als dat van Anna. 
Natuurlijk wil ik ook mijn hele familie bedanken voor de kansen en mogelijkheden die zij mij altijd hebben gegeven om mijn eigen weg te vinden. Zonder jullie bijdrage was dit proefschrift zeker nog niet afgerond. Pa, ik mis je nog elke dag en ik vind het heel erg jammer dat je dit niet meer kunt meemaken. Ma, dank voor al het vertrouwen in me en het thuisgevoel dat iii overal en altijd hebt weten te creëren. Emile, dank je wel voor het altijd klaar staan voor ons. Koen, Reineke, Stijn, Sophie en Emma, dank voor alle steun tijdens de afgelopen jaren.

Anna, samen met jou zijn de afgelopen jaren heel erg goed en fijn geweest. Het is dan ook dankzii jou dat dit promotietraject heeft geresulteerd in een heel positieve ervaring en een mooie periode in mijn leven. En samen met onze Luuk gaan we er ook de komende jaren iets heel moois van maken! 


\section{Curriculum Vitae}

Remco Megens was born on April 5, 1976 in 's-Hertogenbosch, the Netherlands. After completing the school of higher secondary education (HAVO) and studying one year of "food technology and environment" at the AOC Midden \& Oost Brabant (Boxtel, the Netherlands), he started to study "Medical Imaging and Radiotherapeutical Techniques" (MBRT) at the Fontys Hogescholen (Eindhoven, the Netherlands) in 1995. In 1999 he obtained a bachelor degree in Medical Imaging and Radiotherapeutical Techniques. From 1999-2002 he studied Health Sciences, specialisation Movement Sciences, at Maastricht University (the Netherlands), where he obtained a master degree. From December 2002 he started as a PhD-student at the Department of Biophysics of Maastricht University on the project 'Vital Imaging of Large Arteries using Two-Photon Laser Scanning Microscopy: Focus on the Arterial Wall'. Since May 2007 he is working as a post-doctoral researcher at the Department of Physiology of Maastricht University on a 2-year project 'Evaluation of compounds aimed at inhibition of platelet activation/aggregation or coagulation using a high shear rate flow chamber and intravital microscopy models'. For this project he is currently send on secondment at AstraZeneca R\&D, Department of Bioscience, Mölndal (Sweden) for a period of one year. 
Chapter 9 


\section{List of Publications}

\section{Papers}

Megens RTA, Reitsma S, Schiffers PHM, Hilgers RHP, De Mey JGR, Slaaf DW, oude Egbrink MGA, van Zandvoort MAM. Two-Photon microscopy of vital murine elastic and muscular Arteries. Combined structural and functional imaging with subcellular resolution. J of Vasc Res. 2007; 44(2); 87-98.

Prinzen L, Miserus RJ, Dirksen A, Hackeng TM, Deckers N, Bitsch NJ, Megens RTA, Douma K, Heemskerk JW, Kooi EM, Frederik PM, Slaaf DW, van Zandvoort MAM, Reutelingsperger CP. Optical and magnetic resonance imaging of cell death and platelet activation using annexin A5-functionalized quantum dots. Nano Lett 2007;7(1); 93-100.

Douma K, Megens RTA, Reitsma S, Prinzen L, Slaaf DW, van Zandvoort MAM. Two-photon lifetime imaging of fluorescent probes in intact blood vessels: a window to sub-cellular structural information and binding status. Microsc Res Tech. 2007 May;70(5):467-75.

Megens RTA, oude Egbrink MGA, Cleutjens JPM, Kuijpers ME, Schiffers PHM, Merkx M, Slaaf DW, van Zandvoort MAM. Imaging collagen in intact viable healthy and atherosclerotic arteries using fluorescently labeled CNA35 and two-photon laser scanning microscopy. Mol Imaging. 2007 Jul-Aug;6(4):24760.

Boerboom RA, Krahn KN, Megens RTA, van Zandvoort MAM, Merkx M, Bouten CVC.

High resolution imaging of collagen organisation and synthesis using a versatile collagen specific probe. J Struct Biol. 2007 Sep;159(3):392-9.

Baumann M, Megens R, Bartholome R, Dolff S, van Zandvoort MAM, Smits JFM, Struijker Boudier HA, De Mey JGR. Prehypertensive RAAS blockade in SHR ameliorates long-ferm vascular function. Hypertens Res. 2007 Sep;30(9):853-61.

De Mey J, Megens R, Fazzi G. Functional antagonism between endogenous NPY and CGRP in mesenteric resistance arteries. J Pharmacol Exp Ther. 2007 Nov 30; [Epub ahead of print] 
Papers submitted

Megens RTA, oude Egbrink MGA, Merkx M, Slaaf DW, van Zandvoort MAM. Imaging collagen and inflammatory cells in atherosclerotic plaques in vital carotid arteries using two-photon microscopy. Submitted for publication.

Van Zandvoort MAM, Megens RTA, Huisman A, Engels W, van 't Wout K, de Groot H, Sustmann R, Korth HG, van Faassen EE. Imaging enhanced NO levels in stimulated sprouting cultured endothelial cells. Submitted for publication. 

